\title{
Directed assembly and development of engineered tissues using microwell screening platforms
}

Citation for published version (APA):

Vrij, E. J. (2016). Directed assembly and development of engineered tissues using microwell screening platforms. [Doctoral Thesis, Maastricht University]. Maastricht University.

https://doi.org/10.26481/dis.20161201ev

Document status and date:

Published: 01/01/2016

DOI:

10.26481/dis.20161201ev

Document Version:

Publisher's PDF, also known as Version of record

\section{Please check the document version of this publication:}

- A submitted manuscript is the version of the article upon submission and before peer-review. There can be important differences between the submitted version and the official published version of record.

People interested in the research are advised to contact the author for the final version of the publication, or visit the DOI to the publisher's website.

- The final author version and the galley proof are versions of the publication after peer review.

- The final published version features the final layout of the paper including the volume, issue and page numbers.

Link to publication

\footnotetext{
General rights rights.

- You may freely distribute the URL identifying the publication in the public portal. please follow below link for the End User Agreement:

www.umlib.nl/taverne-license

Take down policy

If you believe that this document breaches copyright please contact us at:

repository@maastrichtuniversity.nl

providing details and we will investigate your claim.
}

Copyright and moral rights for the publications made accessible in the public portal are retained by the authors and/or other copyright owners and it is a condition of accessing publications that users recognise and abide by the legal requirements associated with these

- Users may download and print one copy of any publication from the public portal for the purpose of private study or research.

- You may not further distribute the material or use it for any profit-making activity or commercial gain

If the publication is distributed under the terms of Article $25 \mathrm{fa}$ of the Dutch Copyright Act, indicated by the "Taverne" license above, 


\section{Directed assembly and development of engineered tissues using microwell screening platforms}

Erik J. Vrij 
Copyright 2016 ㄷ Erik J. Vrij, Maastricht

ISBN: 978-94-6233-480-9

All rights reserved

The work described in this thesis was carried out in the department of Complex Tissue regeneration of Merln Institute for Technology-Inspired Regenerative Medicine (Maastricht University, the Netherlands) and the department of Tissue Regeneration of MIRA Institute for Biomedical Technology and Technical Medicine (University of Twente, the Netherlands).

This research was funded by the Netherlands Institute of Regenerative Medicine (NIRM) and the Province of Limburg.

The printing of this thesis was sponsored in part by the Netherlands society for Biomaterials and Tissue Engineering (NBTE).

Published by Erik J. Vrij

Cover design: Eva C. Vrij

Printed by: Gildeprint - Enschede 


\title{
Maastricht University
}

\section{Directed assembly and development of engineered tissues using microwell screening platforms}

\author{
Dissertation
}

to obtain the degree of Doctor at the Maastricht University, on the authority of the Rector Magnificus, Prof. dr. Rianne M. Letschert in accordance with the decision of the Board of Deans, to be defended in public on Thursday, December 1, 2016, at 14:00 hours

by

\section{Erik Jacob Vrij}

geboren op 10 november 1983

te Rotterdam 


\section{Supervisor:}

Prof. Dr. C.A. van Blitterswijk

\section{Co-supervisors:}

Dr. N.C. Rivron

Dr. R.T.K. Truckenmüller

\section{Assessment Committee:}

Prof. dr. F.C.S. Ramaekers (chairman)

Prof. dr. ir. A. van den Berg (University of Twente)

Prof. dr. J de Boer

Dr. E.H.J. Danen (Leiden University)

Dr. M. van de Wetering (Hubrecht Institute Utrecht) 


\section{Table of contents}

CONTENTS PAGE

$\begin{array}{lll}\text { Chapter } 1 & \text { Directing Development of Engineered Tissues } & 7\end{array}$

Aims and outline of this thesis $\quad 25$

Chapter 2 Directed Assembly and Development of Material-free Tissues with 33

Complex Architectures

$\begin{array}{lll}\text { Chapter } 3 & 3 D \text { High Throughput Screening and Monitoring of Embryoid Bodies in } & 71\end{array}$

Thermoformed Microwell Plates

Chapter $4 \quad$ Improving a Model of Preimplantation Embryonic Development using $\quad 91$ HCS

Chapter 5 Finding Small Molecule Modulators for Blastoid Development using High-

Throughput Screening

$\begin{array}{lll}\text { Chapter } 6 & \text { General Discussion } & 155\end{array}$

$\begin{array}{lll}\text { Chapter } 7 & \text { Valorization of Research Findings } & 163\end{array}$

$\begin{array}{ll}\text { Summary } & 171\end{array}$

$\begin{array}{ll}\text { Biography } & 173\end{array}$

$\begin{array}{ll}\text { Publication record } & 175\end{array}$

$\begin{array}{ll}\text { Acknowledgements } & 177\end{array}$ 



$$
20
$$


Chapter 1 


\section{Tissue engineering approaches}

Generating tissues in vitro creates significant potential for the field of regenerative medicine as use for medical therapies (replacing damaged tissues), disease modelling and drug screening (replacing animal experiments). Classical tissue engineering often involves the use of scaffold materials, either synthetic or organic in nature, combined with bioactive components such as cells, extracellular matrix (ECM) constituents or growth factors. Such materials can mimic the extracellular matrix and provide increased control over 3-dimensional tissue formation and its mechanical support. More recently however, approaches that use the self-instructive capacity of cells and their ECM constituents to build tissues and organs have gained more momentum. Here, an introduction is outlined about the technological and biological advancements that push the field of regenerative medicine towards exploiting methods of tissue-authentic and organotypic culture and how to direct and image these cultures in high-throughput.

\section{Top-down tissue engineering}

In the classical design, tissue engineering pursues to restore function of tissues through the combination of cells, bioactive molecules and (bio)materials, of which the latter can provide molecular and mechanical signals to support cell adhesion, differentiation, proliferation and secretion of ECM. At the same time these biomaterials act as a matrix template (i.e. scaffold) and offer structural support for the tissues that are formed. Ideally, when the biomaterial is biodegradable, the mechanical properties of the tissue engineered construct are transiently taken over by the natural ECM that is secreted by the cells and which remodels the newly formed tissue.

Scaffold materials, including polymers and ceramics, can be tailored by adjusting the chemical (e.g. hydrophobicity), physical (e.g. topography), and mechanical properties (e.g. elasticity) of the material to tune the cell-material interface, release of factors or degradation kinetics ${ }^{1}$. Scaffold elasticity (stiffness) has shown to be an important parameter in modulating tissue development ${ }^{2}$. Thus, the appropriate elasticity highly depends on the ECM elasticity of the tissue type that the scaffold is designed for ${ }^{3}$. Opportunely, native (or autologous) ECM can also be used as a bioactive scaffold, either in its original form or reconstituted. Such ECM bio-scaffolds support constructive tissue remodeling, however, clinical applications are found mainly in soft tissues, such as dermal and vascular tissue reconstruction ${ }^{4,5}$.

Although scaffold-based approaches have been successfully translated to the clinic, several drawbacks remain, such as limited spatial control over the cellular microenvironment and pore connectivity associated with poor cell distribution and nutrient diffusion. Moreover, the majority of our tissues, show complex non-linear behavior. For example the factors that are locally released to fine-tune tissue development in a temporal and spatial manner or the anisotropic mechanical behavior ${ }^{6}$, which is challenging to mimic using classical fabrication methods. Lately, these issues are approached by novel additive manufacturing processes and techniques, such as 3D printing, which provide substantial control over scaffold architecture, which can be employed for example to pattern a vascular network within a matrix of other cell types ${ }^{7}$. 


\section{Bottom-up tissue engineering}

Native tissues consist of highly hierarchical structures, which are often comprised of repeating organized multicellular units that act in concert to establish a mesoscale function. Bottom-up tissue engineering aims at building tissues by assembling multiple-scaled cellular building blocks with microstructural features mimicking native tissue architecture. Currently, several strategies for bottom-up tissue engineering are actively studied (reviewed in ${ }^{8-10}$ ). These include the assembly of cell sheets ${ }^{11}$, cellular aggregates ${ }^{12}$, cell or aggregate-loaded hydrogels ${ }^{13,14}$ and the combination of engineered micro-objects with cells to build 3D tissues ${ }^{15}$. Fused tissues can be achieved by directed self-assembly using geometrical docking of building blocks in hydrogel templates ${ }^{16}$ or within microfluidic chips ${ }^{17}$, or by crosslinking ${ }^{18}$ or base-pairing of single-stranded DNA (DNA origami) ${ }^{19}$ amongst many other options. Moreover, in succession of printing scaffolds, 3D printing is also emerging as an appealing tool to print cells, aggregates and corresponding ECM ${ }^{13,20-22}$. Bottom-up approaches are generally characterized by their high spatial control over tissue structure and upscaling potential. As of this, tissue subunits with a uniform three-dimensional distribution of cells can be hierarchically assembled, hereby overcoming the limits of top-down fabrication methods.

\section{Material-free approaches using self-assembly}

Many bottom-up strategies involve some sort of (bio)material to provide additional structure to the tissue. Although biocompatible, such foreign materials might lead to unfavourable degradation products leading to a variety of adverse effects such as inflammation and fibrous tissue formation ${ }^{23}$. Moreover, the addition of materials in generating tissues in vitro might restrict their remodelling capacity including the highly organized microscale structures that guarantee proper tissue function. Although hydrogel materials allow a certain degree of remodelling, these restraints are predominantly present in hard-material scaffolds (top-down).

In contrast to scaffold-based approaches, cells can also form scaffold-free tissues by their capacity to aggregate into three-dimensional spheroids by self-assembly, which is a fundamental process underlying the shaping of tissues and organs including their structural organization ${ }^{24,25}$. This feature of self-assembly follows in part from the differential adhesion hypothesis, which describes the liquid-like behaviour of cells when they adhere, sort, spread and segregate as a physical expression of the cell populations' diminution of adhesive-free energy ${ }^{26,27}$. Spheroids resemble in vivo tissues more closely than cells grown in two dimensions. Hence, cellular phenomena like ECM deposition, spreading, migration and polarization manifest in a more physiological manner, which affects macro-cellular processes such as the transport of soluble factors or the transmission of mechanical forces through integrin clustering ${ }^{28}$. The two central recognized forms of scaffold-free tissues formed by self-assembly are spheroidal microtissues (i.e. spheroids) ${ }^{29}$ and cell sheets ${ }^{30}$.

\section{Heart}

Microtissues may prove beneficial in stem cell therapies using cell engraftment to regenerate tissues. One example is the heart, which shows a limited tendency to regenerate in ischemic cardiovascular diseases, such as myocardial infarction ${ }^{31}$ and is thus an interesting target for stem cell therapies to enhance repair and cardiac function. A prominent obstacle in such therapies is the delivery and survival of implanted stem cells, which can be improved by engrafting spheroids from cardiac progenitor cells ${ }^{32}$. 


\section{Cartilage}

Cellular aggregates are already an established means in cartilage tissue engineering as it was found that the chondrocyte phenotype is better preserved in 3D spheroids than in 2D monolayer culture ${ }^{33}$. The ECM that chondrocytes deposit within these spheroidal microtissues recapitulates the physiological characteristics of cartilage more closely in terms of matrix composition, density and macrostructure ${ }^{34,35}$. Also, multiple cell types can be combined in spheroid cultures which may benefit cartilage development by the release of trophic factors. Hence it was shown that coculture of chondrocytes with mesenchymal stem cells (MSCs) increases proliferation and ECM deposition of chondrocytes ${ }^{36}$. Moreover, spheroids from human MSCs can be shifted in their differentiation route towards an articular or hypertrophic cartilage phenotype by modulating the oxygen concentration ${ }^{37}$. This indicates that gradients of oxygen can contribute to the heterotypic differentiation of stem cells within microtissues.

\section{Bone}

Cellular aggregates may also serve in a hybrid approach with a biomaterial. For instance, as study showed that an effective strategy for bone tissue engineering from embryonic stem cells includes an initial step of spheroid (i.e. embryoid body) formation followed by seeding them on ceramic particles ${ }^{38}$. Through this step of spheroid formation, and the addition of growth factors, the cells were coaxed through a programme of endochondral ossification in which the cells deposit a cartilage matrix that serves as a template for the formation bone.

\section{Cell-sheet technology}

The Okano group pioneered the generation and stacking of cell sheet constructs to build prevascularized modular tissues ${ }^{39}$. Here, the release of cell sheets is controlled by culturing them on top of a thermoresponsive polymer poly-(N-isopropylacrylamide) (pNIPAAm) based hydrogel, which allows lifting off the newly grown cell sheet by altering the temperature. In contrast to methods of using enzymatic dissociation, these cell sheets retain their secreted ECM and cell-cell junctions. Untethered cell sheets can be further manipulated by wrapping, stacking or rolling for instance ${ }^{30}$.

\section{Exploiting microfabrication technology for tissue engineering}

\section{Lithographic patterning}

Micro- and nanofabrication technologies, which were originally developed for the microelectronics industry, allow designing and forming multileveled structures with precise sizes and shapes down to nanometer scale. Lithographic patterning through photomasks is one particular technique that is commonly used to create structures in material substrates for cell and tissue culture. Adaption of this technology led to the replication of micro- and nanoscale features into a wide array of biocompatible materials. Different techniques exist to replicate the geometries obtained through lithographic patterning techniques, such as hot embossing, microthermoforming, injection moulding or replica moulding, of which the latter has arguably contributed the most to advance the field of 3D microculture techniques. Replica moulding allows to geometrically and chemically manipulate the microenvironment while maintaining upscaling possibilities ${ }^{40}$. For example, geometrical microstructures can be used to guide tissue formation, such as sprouting of new capillaries from endothelial cells ${ }^{41}$. Hydrogels are often the material of choice as they closely 
mimic native tissue ECM and they permit the allocated encapsulation of cells or biomaterials to specific compartments in 3D structures ${ }^{42}$. Another popular approach is the patterning of microwell templates into hydrogel for the controlled but autonomous assembly of cells therein ${ }^{43-45}$.

\section{Microfluidics}

Microfluidic approaches are emerging as a means to integrate miniaturization with extended sample processing and rapid analysis ${ }^{46}$. For instance, microfluidics is able to provide controlled gradients of soluble and physical cues that affect the cellular microenvironment by generating an endless number of configurations ${ }^{47}$. In particular, microfluidic systems possess the intrinsic advantage of increased control over diffusion; minimally hindered by convection. Hence, the integration of microfluidics with tissue engineering, including an accessible read-out, allows studying the cellular microenvironment in great detail. For example, tumour-mimetic environments can be used to model tumour angiogenesis by applying gradients of soluble factors ${ }^{48}$. Another illustrative benefit of microfluidics is to study tissue and cellular interfaces, for example the effect of strain and mass transport in the alveolar-capillary interface of human lungs ${ }^{49}$. Such platforms are already being transformed to permit compatibility with 3D cellular models in which concentrations of soluble factors such as morphogens can be accurately administered and studied for their effect on development ${ }^{50,51}$. Translating microfluidic systems to $3 \mathrm{D}$ cell models is critical to have tight spatiotemporal control on cell fate decisions and morphogenetic events.

\section{Assembly of spheroids into larger tissues}

It has been demonstrated that spheroidal microtissues formed by cellular self-assembly can recurrently be used in a process of confined self-assembly to form larger-sized shaped tissues with high cellular density ${ }^{52}$. However, these tissues often lack the mechanical integrity to support tissue maintenance and growth and therefore deform over time resulting in loss of shape and tissue organization ${ }^{53}$. Yet, it is suggested that pre-processing of spheroids by mixing in ECM components, conditioning them with physical stimuli such as pulsatile flows or the incorporation of magnetic nanoparticles may give increased control over spheroid maturation and thus of the tissue macroorganization ${ }^{54}$.

\section{Directing development in vitro}

The in vitro generation of tissues and organs including all corresponding cell types and their complex structural organization is a major challenge in the field of tissue engineering. The inherent complexity of biological systems is often oversimplified in approaches to controlling tissue formation in vitro. Living materials consist of adaptive multi-protein networks that provide instructive roles for the cells they embed. Fabricated scaffolds can mimic certain crucial aspects, both structurally and instructively, but do not recapitulate the dynamic complexity of native tissues.

A new wave in generating tissues in vitro involves harnessing the power of developmental mechanisms. This methodology is based on guiding tissue formation through sequential stages reminiscent of the stages of native development. Characteristic for this approach is gradual tissue growth including progressive cellular differentiation and morphogenesis such that the final tissue matures through intermediate tissue forms. 
All organs are made up of tissues derived from the three embryonic germ layers, which are the endoderm, mesoderm and ectoderm. The endoderm constitutes the epithelium of lungs and digestive organs, the mesoderm gives rise to tissues of the musculoskeletal system and mesenchymal connective tissue, and the ectoderm generates the nervous system and skin epithelium including the tissues derived from it such as the mammary gland. During organogenesis, cells of epithelial and mesenchymal origin interact and collectively coordinate tissue morphogenesis and differentiation ${ }^{55}$. Hence, as was already studied almost hundred years ago ${ }^{56}$, development of individual tissues and organs does not occur autonomously and in isolation but is instructed by physical (geometry, forces) and chemical instructions from the surrounding tissues.

\section{Directed differentiation and development}

Pluripotent stem cells have the capacity to form all functional tissue-specific cell types and could previously only be obtained from preimplantation embryos as embryonic stem (ES) cells. The realization of induced pluripotent stem (iPS) cells has opened up new avenues for patient-specific regenerative medicine approaches, boosting the interest in directed differentiation. Generating enriched populations of tissue-specific cell types holds promise for drug discovery, predictive toxicology, functional human disease modelling and clinical applications. Meanwhile, by employing the right soluble factors a plethora of tissue-specific cell types can be generated, such as hepatocytes ${ }^{57-59}$, lung airway epithelial cells ${ }^{60}$, pancreatic beta cells ${ }^{61}$, chondrocytes ${ }^{62}$ or hematopoietic stem cells, which are the most widely transplanted stem cells in the clinic thus far ${ }^{63}$.

A common approach to direct the differentiation of cells is the use of high-throughput screening (HTS) of growth factors and small molecules. Using HTS methods precise protocols can be derived, including the administration of factors at functional concentrations and time lengths, that drive cells through a sequence of pathways active in development, into a homogenous population of a specific lineage ${ }^{64}$. Often, tissue-specific differentiated cell types cannot be expanded in culture or lose their functionalities. As such, the derivation and expansion of tissue-specific stem cells or progenitor cells can be an attractive substitute. The protocols to do so often include small molecules in combination with growth factors to modulate developmental pathways such as Wingless (Wnt) / $\beta$-catenin, transforming growth factor (TGF) - $\beta$, receptor tyrosine kinase (RTK) /MAP kinase, Hedgehog or Notch signalling ${ }^{65-68}$. Cell therapies involving directed differentiation may find applications in replacing lost or damaged cells with healthy substitutes, induce regeneration via paracrine signals or help in finding chemical or biological substances that target cells in vivo to restore function or regenerate tissues (Figure $1^{69}$ ). An interesting example is the stable expansion of mesenchymal progenitor cells from iPS cells that may alleviate existing potential clinical applications for human mesenchymal stem cells ${ }^{70}$. However, the purity or homogenous identity of progenitor/ stem cells remains a challenge that need to be overcome. 


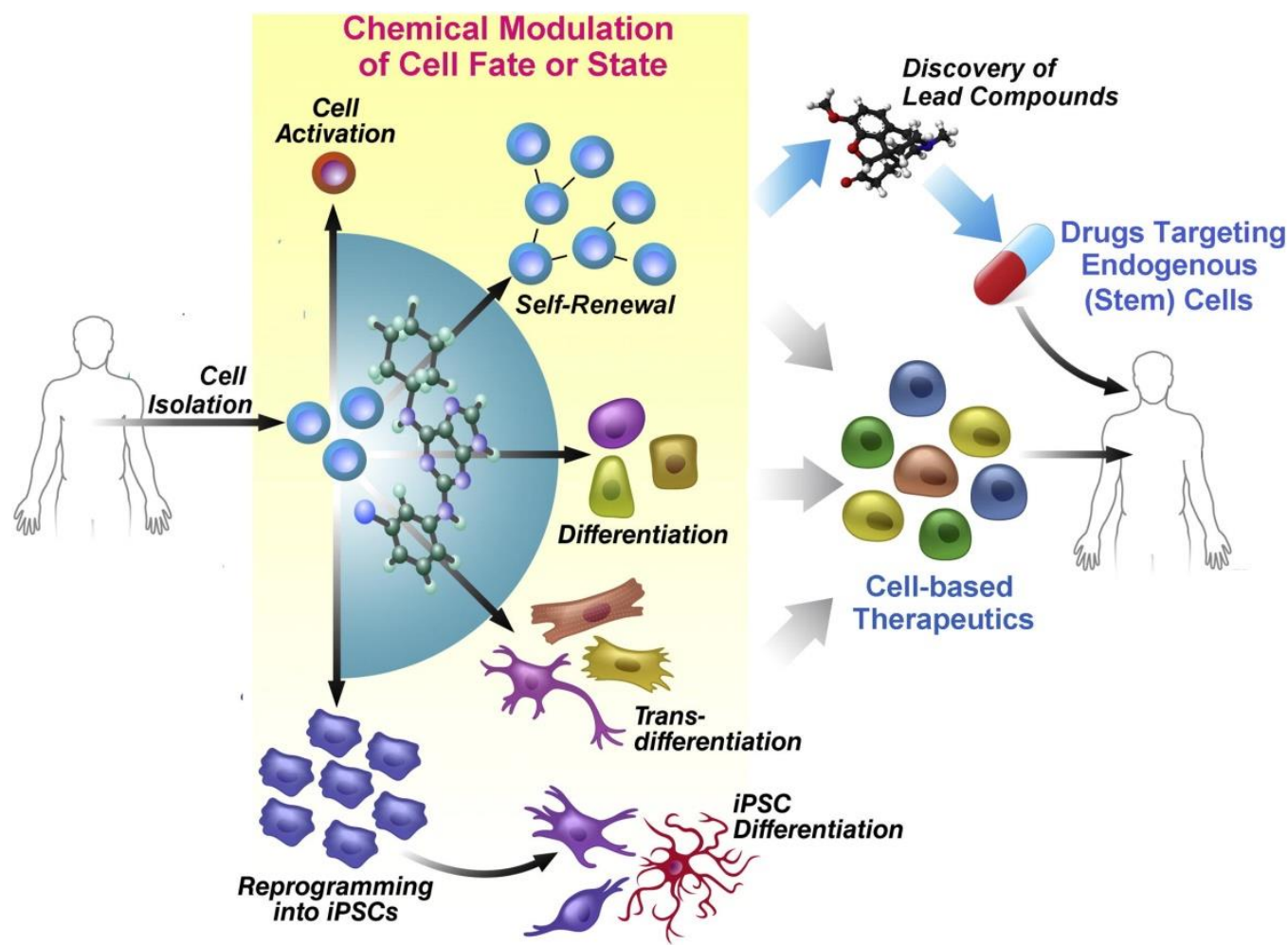

Figure 1: Chemical factors are screened and applied to drive cell fate towards specific lineages. The cells that are generated by methods of directed differentiation can be used in cell-based therapy or to further develop therapeutic chemicals manipulating endogenous cells in the patient targeting disease or injury. Figure adapted from ${ }^{69}$.

\section{The advent of in vitro generated organotypic tissues}

Recently, culture of 3-dimensional multicellular models of organogenesis experiences a thriving growth of interest in the field of regenerative medicine (a non-comprehensive overview of organoids including their size in Figure 2, reviewed in ${ }^{71-73}$ ). In order to regenerate the full spectrum of tissuespecific cell types in an organ model, including all transient (short-lived) precursors, complementary signals resulting from the $3 \mathrm{D}$ microenvironment may be required, such as cell-ECM interactions, polarization and physical forces - aspects that are generally neglected in $2 \mathrm{D}$ culture. As such, inspiration from developmental programs in biology led to a new research field, so-called developmental (bio)engineering, which aims at regenerating functional tissues and organ models in vitro through pathways archetypical for native development. These organ models, termed organoids, are capable of self-renewal and self-organization and show comparable, nearphysiological organ functionality in vitro. Methods of screening for soluble factors and regulating physical stimuli that affect development and direct organogenesis can result into more functional models. The physiological relevance of such in vitro-generated cell-based constructs is key and can prove useful in assays for personal medicine or applications in tissue engineering. Additionally, organoid cultures can advance the quantitative modeling of biological systems including systems of multiple cells, tissues and even organisms. Of equal importance, the increased resemblance to native tissues improves predictive power in drug and toxicology assays, which has the potential to reduce the number of animal experiments conducted in research institutions and pharmaceutical practices. 
Combining this technology with induced pluripotency stem (iPS) cells and adult stem cells obtained from individual patients, tissues and organ models can be formed that match genetically, and thus permit a personal approach on disease analysis and therapeutic study. Novel genome editing technologies, such as CRISPR/Cas9 ${ }^{74}$, help in revealing the genetic basis of individual DNA modifications involved in disease phenotypes, and may contribute to treatment strategies.

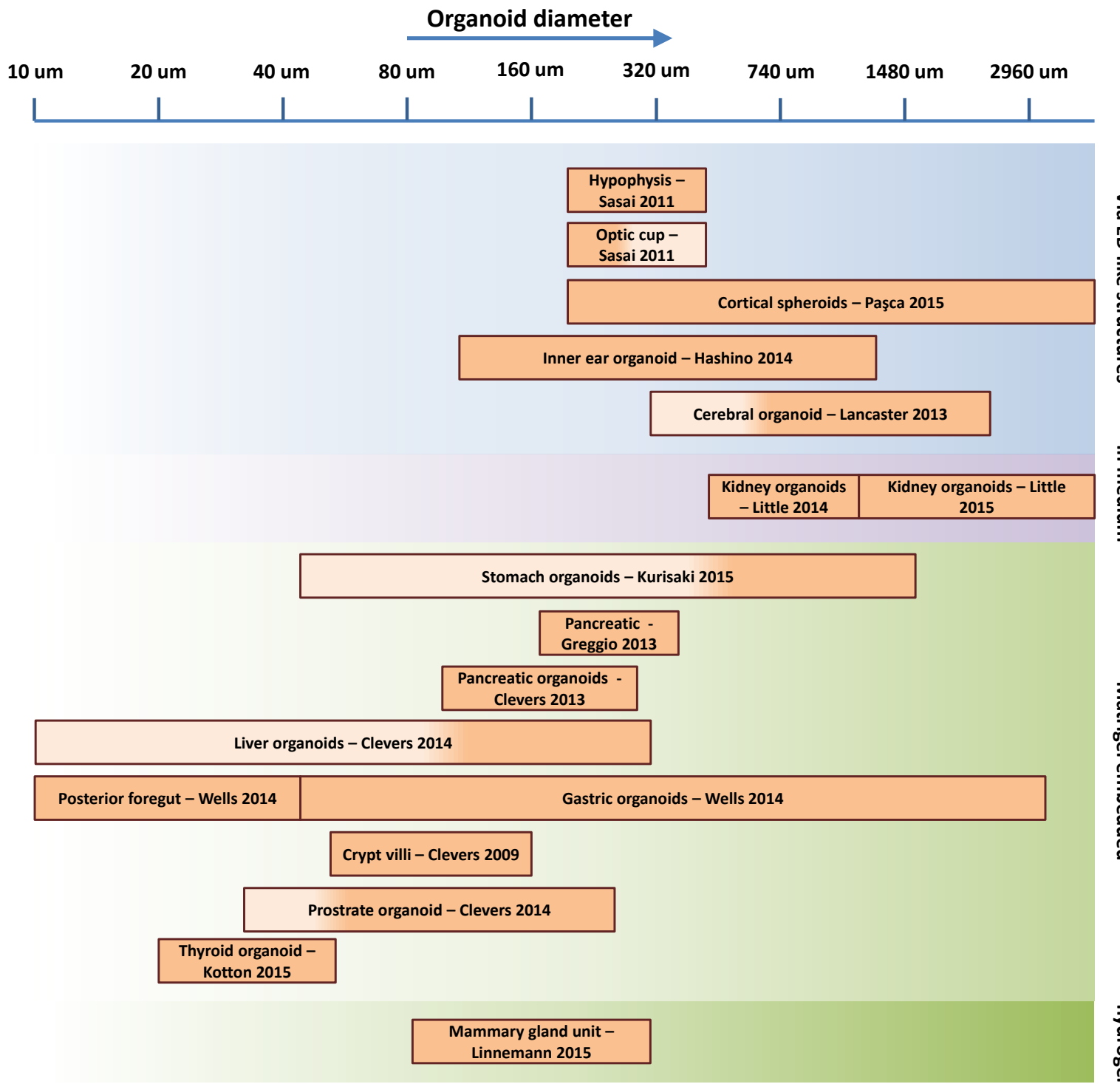

Figure 2: Various recent in vitro organoid models displayed according their size (diameter) and culture format. 


\section{A nexus of directed differentiation and self-organization}

Organoids can be generated using multipotent stem cells obtained from primary tissues or using pluripotent stem cells obtained from blastocysts (embryonic stem cells) or by induced pluripotency (iPS cells). By providing the right directional cues, the cells can structure themselves in an organotypic manner through either a monolayer growing on a 2D surface, by aggregation into celldense spheroids or by embedment in a 3D hydrogel (often Matrigel, see Figure 2). Rock inhibitors, such as y27632, are commonly used to facilitate cell clustering and inhibit apoptosis after cell dissociation.

\section{Generating organoids from multipotent stem cells}

Organoids produced from dissociated multipotent stem cells typically arise while being embedded within a hydrogel comprised of ECM-components supplemented with organoid-specific niche factors in culture medium. The hydrogel-based artificial ECM often consists of collagen, fibronectin, laminin or Matrigel, of which the latter contains a heterogeneous mixture of extracellular proteins. In general, the ECM components within the hydrogel provide the supporting structural signals for correct multicellular organization.

Meanwhile, a steadily increasing number of organs find their organoid equivalents generated in vitro. Landmark studies by Clevers et al. almost a decade ago greatly contributed to the gain of momentum in this field of research ${ }^{75}$. Here it was shown that Lgr5+ stem cells from the intestinal crypt can renew the full intestinal epithelium ex-vivo, including its characteristic 3D structure ${ }^{76}$. Lgr5+ stem cells, which permit the binding of the Wnt-agonistic R-spondin ligands to their receptor, were then found in multiple adult organs and could be exploited to regenerate corresponding organoids. Signalling factors from the stem cell niche that play an important role in the regeneration of organs, such as R-spondin, epithelial growth factor (EGF) and Noggin are essential in forming intestinal organoids. Enriching the stem cell niche by a Paneth cell/ Lgr5+ stem cell combination greatly improves the organoid formation yield, which stresses the significance of paracrine and juxtracrine signals in stem cell activation. Likewise, Lgr5+ stem cells from the liver bile duct were induced with factors R-spondin, EGF, hepatocyte growth factor (HGF), fibroblast growth factor (FGF)10 and nicotinamide to form cystic organoids capable of differentiating to the functional hepatocyte lineages ${ }^{77}$. Other organ-types include the taste bud ${ }^{78}$ and the stomach ${ }^{79}$.

\section{Directing differentiation and development of pluripotent cells}

Directing the differentiation of pluripotent stem cells for organoid culture typically requires their funneling through the appropriate germ layer before exposing to tissue-specific patterning factors. For endoderm derived organoids, TGF- $\beta$-related signaling is usually stimulated in pluripotent stem cells to drive them first to definitive endoderm, either in monolayer cultures or in embryoid bodies 80, 81 . Cells are then coaxed further in development by temporal manipulation of activators and inhibitors of classical developmental signaling pathways (see Figure 3), such as EGF, FGF, Wnt, retinoic acid and TGF- $\beta$ superfamily signalling pathways including Nodal, bone morphogenetic protein (BMP) and Activin. More specifically, for instance in gastric organoids, Wnt, FGF and Noggin are activated following on definitive endoderm induction, which then leads to the autonomous formation of foregut spheroids. Organoid culture is then continued within a matrigel matrix complemented with EGF, Noggin and retinoic acid signaling activation ${ }^{82}$. Other endoderm-derived 
cells that exhibit organoid forming abilities include progenitor cells for liver ${ }^{83}$, pancreas ${ }^{84,85}$, intestine ${ }^{86}$, thyroid ${ }^{80}$ and stomach ${ }^{87}$.

Alternatively, the number of successful organoids derived via mesoderm is still limited. However, recently renal organoids were generated ${ }^{88,89}$ through a mesodermal state by modulating Wnt and FGF signaling ${ }^{90}$. These kidney organoids comprised of up to 500 nephrons including collecting ducts surrounded by endothelia and renal interstitial cells. 


\section{Directed differentiation of pluripotent embryoid bodies}

Organoids coaxed through the ectoderm layer are typically induced by forming embryoid body (EB)-like aggregates, which are then further directed into their neural or non-neural progeny. This method has been used in combination with defined ECM components to form structures recapitulating the eye cup including retinal regions ${ }^{91}$. Additionally, brain organoids have been developed resembling the multilayered cerebral cortical structure ${ }^{92}$ and similar organoids were used to model microcephaly, a brain disorder ${ }^{93}$. In another study an epithelia with mechanosensitive properties reminiscent of the inner ear was formed including hair cells, supporting cells and sensorylike neurons ${ }^{94}$. Furthermore, large EBs, consisting of around ten thousand cells, have been directed to self-organize into pituitary gland-like structures that functionally secreted the adrenocorticotropic hormone in response to a physiological stimulus and thereby rescued systemic glucocorticoid levels in hypopituitary mice ${ }^{95}$. Such studies in mouse have proven valuable for translation to human, as the same group recently demonstrated a similar regulative response in human pituitary analogues ${ }^{96}$.

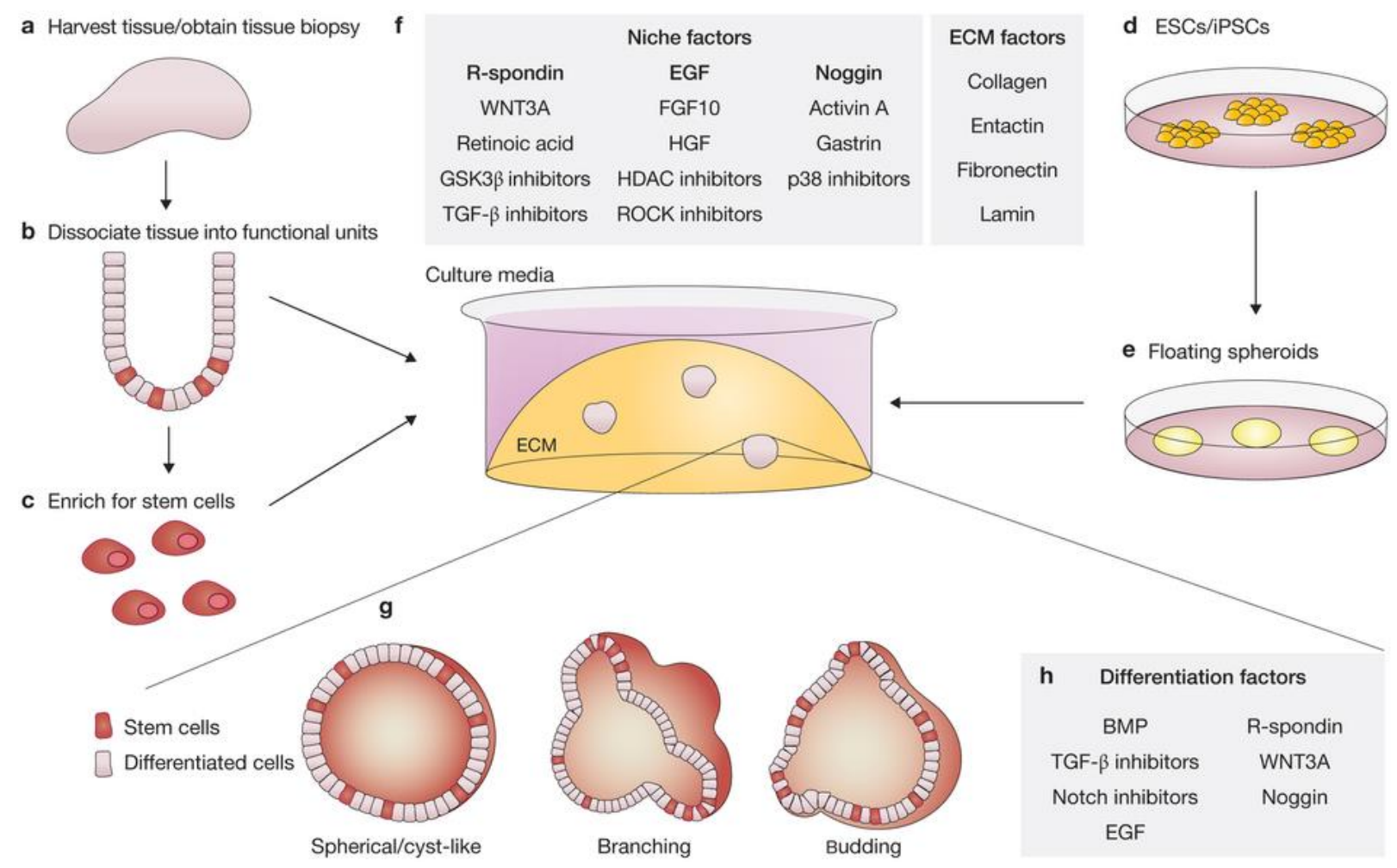

Figure 3: Organoid formation from adult (progenitor) stem cells involves the appropriate culture conditions that modulate developmental signalling pathways such as ECM components and soluble factors, either supplied by the niche or exogenously added. Pluripotent stem cells are direct ed in their germ lineage differentiation from which they form aggregates either before or after being embedded within a hydrogel containing ECM components or by continuously free-floating. Organoids then develop spontaneously or induced by differentiation factors. Stem cells are propagated within organoids and continually produce their differentiated progeny. Figure adapted from ${ }^{71}$. 


\section{Benefits and limitations of organoid culture}

Recent attempts at recapitulating organogenesis in 3D cultures have unlocked new important possibilities in pharmaceutical development, regenerative medicine and basic biological research. For example, studying processes of development and disease in vitro in various contexts is more amenable with near-physiological models. This accounts especially for human diseases that are difficult to model in animals. Opportunely, these models can be commonly generated from a limited number of cells or starting material that are often easily obtained from a single patient during surgical procedures. Organoid cultures also enable the propagation of stem and progenitor cells including their downstream tissue-specific lineages with minimal supplemented factors, as stem cells are maintained and propagated in their niche within these organoids, while continually generating differentiated progeny.

Limitations also exist in organoid cultures. For example, and in contrast to other tissue engineering approaches, incorporation of a blood supply lacks so far, which limits the size of these cultures and their propagation towards full grown mature organs. Also, modelling inflammatory responses is difficult due to the absence of immune cells in these models. Equally, cells from surrounding stromal tissue are often absent and thus their potential influence is neglected. Other challenges include the yield in organoid formation and the heterogeneity in terms of developmental potential, viability, size and shape within the same culture or experiment, which hampers reliable statistical significant read-outs. Upscaling in combination with automatized and smart selective imaging could overcome such issues on the side of analysis.

\section{Impact of organoid cultures}

Organoid systems have the potential to model diseases, such as genetic disorders; being used for toxicology studies; and for regenerative medicine purposes. A promising application is the use of patient-derived organoids that can be implanted an revive organ function. Some of such applications may find the clinic in the near future, for example the functional differentiation of hepatocytes upon implantation in vivo ${ }^{97}$.

\section{Personalized medicine}

In an approach of disease modelling, patient-material derived organoids can be used to probe the molecular features of neoplastic progression in vitro, for which personalized medicine can be identified and used to treat cancer. The first steps with such approaches are being made, for example for pancreatic ${ }^{85}$ and intestinal cancer ${ }^{98}$ and cystic fibrosis. With such technology, the right combination within a set of therapeutics could be tested within a limited time window and then be administered to the patient. Moreover, organoid biobanks are meanwhile being created to identify gene-drug associations ${ }^{99}$. Such approaches may provide an intermediate between cancer genetics and patient trials and therefore an alternative in personalized therapy to resource-intensive tumour xenograft models.

\section{Gene therapy}

Gene functions in specific organs can be dissected by recapitulating organogenesis from genetically modified progenitor or stem cells ${ }^{100}$. Alternatively, gene editing can be used as a therapeutic means. It was shown that intestinal stem cells from patients with cystic fibrosis can be 
cultured in vitro upon which the single-gene hereditary defect leading to the disease was genetically corrected using CRISPR/Cas9 genome editing. When these corrected stem cells were then directed to form intestinal organoids these showed again a healthy physiological response ${ }^{101}$.

\section{Cell type discovery}

Alternatively, such organoid systems can be used to study development and function of organs and the identity of their cellular constituents. For example, screening and computational analysis on cells from in vitro formed intestinal crypts has been used to discover new rare cell types (Grun et al. 2015).

\section{Image-based screening on multicellular organization}

Phenotypical readouts, including non-invasive morphometric read-outs (e.g. morphology of nucleus, cell, population of cells) and fluorescence markers (e.g. genetic reporters, stainings) are related to the cellular identity and tissue organization ${ }^{102,103}$. High-content analysis (HCA) in cellbased assays is a type of phenotypic screening used in biological research and drug discovery to identify or classify regulators of cell behavior, such as topographies ${ }^{103,104}$, small molecules ${ }^{105,106}$, biomaterials ${ }^{107-109}$, or RNAi ${ }^{110}$. Phenotypic imaging is typically done using light microscopy techniques such as phase-contrast, fluorescence microscopy or a combination of both. As such, HCA necessitates the use of high-content imaging $(\mathrm{HCl})$ systems to capture large amounts of visual data. Miniaturization and upscaling of 3D culture platforms combined with compatibility with $\mathrm{HCl}$ systems allows high-volume and automated analysis of cultures. Thus, this brings high-content analysis of multicellular systems, such as tissues and organoids, to the arena of high-throughput screening, which is appropriately termed high-content screening (HCS). Microfabricated platforms offer new possibilities including more spatial control in patterning cells and modifying the physicochemical properties of the cellular microenvironment. These features can be exploited for high-throughput generation and profiling of organoid models.

\section{HCS on multicellular interaction}

Organoid development in vitro relies on the proper orchestration of pluripotent or tissueprogenitor stem cells in order to form the correct specification of cell types and desired morphogenetic features. This is highly dependent on signaling within the stem cell niche, which include juxtacrine signals (cell-cell contact) or close-range soluble signals ${ }^{111}$. More sophisticated multicellular culture systems, facilitated by microfabrication techniques, allow visualizing the interaction of cells or cell populations and thus offer new insights into the dynamics of collective cell-decision making ${ }^{112}$. For example, a recent study used microfabricated arrays to controllably form heterotypic cell-cell interactions using DNA-programmed adhesion to study juxtacrine signalling in stem cell maintenance ${ }^{113}$. This study involved relative simple $2 \mathrm{D}$ culture of stem cells, however, the same technique of DNA-programmed assembly can also be used to reconstitute the multicellular organization of organoid-like tissues ${ }^{114}$. 


\section{D tissues and organoids}

An established approach of screening 3D tissues is the use of in vitro generated multicellular tumor spheroids to model drug responses in a tumor microenvironment. These tumor spheroids formed from malignant cell lines and patient-derived samples recapitulate the architecture and pathophysiology of tumors in a more authentic way than conventional 2D monolayer culture. $\mathrm{HCl}$ is employed to characterize the effect of drugs on viability and morphology of these cancer spheroids ${ }^{115}$. For example, 3D tumor models show differential sensitivity to anti-cancers drugs. This chemosensitivity is altered by the addition of various non-tumor factors such as the co-culture with fibroblasts or endothelial cells ${ }^{116}$. This highlights the importance of using models with increased complexity in faithfully recapitulating in vivo circumstances.

Yet, the read-out of tumor spheroids and their biological complexity are relatively simple compared to tumor organoids, such as the intestine organoids for modeling colorectal cancer ${ }^{117}$. At the same time, organoids are more cumbersome and costly to form as there are often multiple recombinant proteins applied in high concentrations. Microscale 3D culture systems can provide more cost-effective solutions by reducing culture volumes and reagent needs ${ }^{118}$. Moreover, simplifying the maintenance on 3D cultures and enabling compatibility of novel 3D culture platforms with automated imaging systems is crucial to advance 3D high throughput and high content imaging. Some novel microfabricated platforms already allow screening spheroids or spheroid-based organoids in a HTS-compatible fashion, although throughput is still limited ${ }^{119}$. A solution may be found in adapting microwells to high-throughput compatible well-plates.

\section{Identifying molecular mechanisms using high-content screening}

3D screening approaches may lead to significant acceleration in developing self-organizing tissues in vitro by identifying bioactive compounds that can replace expensive growth factors or ECM (e.g. Matrigel) components. Finding defined components to replace factors of animal origin such as Matrigel or proteins could enable transplantation into humans by complying with regulations. Furthermore, organs that are yet challenging to model (such as the ovary or islets of Langerhans) can be screened for small-molecule modulators of previously unassessed molecular pathways and hormones. In such a way, novel culture components may be discovered that help understanding essential niche factors and facilitate organoid culture. In turn, advancements in organoid culture enable discovery of medically relevant drugs (e.g. small-molecules) potentially elucidating novel molecular mechanisms important in tissue maintenance, homeostasis and disease ${ }^{120}$.

\section{Microscopy of multicellular tissues and organoids}

Automated microscopy systems provide a resourceful means complementing genome-scale biochemical techniques to study biological processes at large scale. Genetic labeling with fluorescent probes has been of great benefit in elucidating signaling pathways, including the movement and trafficking of intracellular proteins, and tracking of live cells in great precision.

Advanced imaging systems based on epi- and confocal fluorescence microscopy allow in situ capturing of the cellular organization within 3D tissues. An excellent signal-to-noise ratio is in all cases essential, which limits 3D imaging in cellular-dense tissues to a couple of hundred micrometer in size, such as gut and liver organoids ${ }^{121}$. To this end, fluorophores with low excitation levels are desired ${ }^{122}$. For dye-coupled immune-detection there is a range of bright photo-stable compounds to 
choose from. More useful for time-lapse live imaging are genetically encoded fluorescent reporters, for instance histone $\mathrm{H} 2 \mathrm{~B}$-fused fluorescent proteins ${ }^{123}$ that facilitate tracking individual cells within a population over time. The fluorescent protein of choice, such as TdTomato ${ }^{124}$, has preferably a high quantum yield and extinction coefficient which correspond to a high brightness. Yet, imaging cellular clusters of larger size is often associated with light absorption and scattering, which compromises image definition.

\section{Light sheet microscopy}

To image larger samples in high resolution with low noise and minimal photo-damage, which can be important in imaging living embryos for example, light sheet fluorescence microscopy is a preferred method ${ }^{125}$. Especially in combination with a deconvolution and spectral un-mixing algorithms this microscopy technique has high potential to digitally map samples in $3 D^{126,127}$. In light sheet microscopy only a thin slice of the sample is illuminated which makes it very suitable to acquire image stacks ${ }^{128}$. For instance, tissues and organoids encapsulated in agarose gel can be conveniently imaged using light sheet microscopy ${ }^{129}$. However, more complex culture substrates such as microwell arrays are more challenging to handle. As the light that excites fluorophores within the specimen enters perpendicular to the path of the observer, the culture platform design has to be adjusted appropriately to allow imaging in high-throughput.

\section{HCA - Image processing and identification}

The human eye is a trained tool to detect patterns and features in images. However, for high content screening and quantification automated machine-based analysis easily exceeds the eye in terms of accuracy and consistency. Free, open-source bioinformatics software tools such as ImageJ ${ }^{130}$, KNIME ${ }^{131}$ and CellProfiler ${ }^{132}$ enable researchers to quantitatively measure features in their images. Objects of interest, such as organelles, cells, colonies of cells, organoids or whole-organisms (e.g. C. elegans worms) can be identified and measured. A collection of measurements can be obtained for each object including morphology, light (fluorescence) intensity and texture. Other key data include the relative position of objects to each other and temporal relations (e.g. cell division). To a certain degree these software packages function as end-point tools as they often provide means for statistical data processing, visualization and analysis.

In a similar fashion, morphogenetic features, comprising the macro-morphometries of collections of cells, can be identified and classified by software tools ${ }^{133}$. One could think of features such as budding, blebbing, or cavitation, including the localized expression of fluorescent reporters or markers (Figure 4). Conversely, employing static algorithms and predefined processing rules that identify such complex phenotypes can be very challenging. The human eye and brain do excel in such complex phenotype identification, such as the cellular organization in tissues and organoids. However, obviously, the human eye provides no answer to the massive amounts of data that are produced in high-throughput screening. For such applications, machine learning methods can assist in analysis such as object identification, (time-resolved) morphometric description and phenotype classification, either by supervised or unsupervised methods ${ }^{134,135}$. 
Examples of 2D projected morphogenetic features

Single cell type:

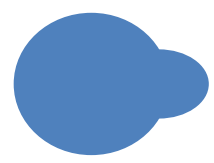

Budding

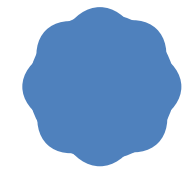

Rugosity

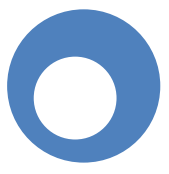

Cavitation

Mixing marked cell types or fluorescent reporters:

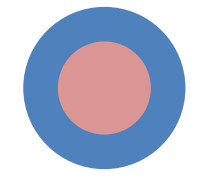

Encapsulation

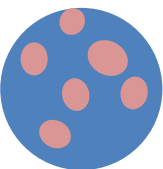

Dispersion

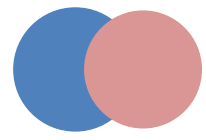

Seggregation

Figure 4: Examples of multicellular morphogenetic readouts.

\section{The next step: Automated volumetric segmentation}

Recently, some efforts are made in reproducibly segmenting tissues in single cells from 3D images acquired through image stacks ${ }^{136}$. An example of applying an HCS methodology to gain new insights in developmental mechanisms in organoids demonstrated that stem cells within the intestinal niche transmit short-range Wnt signals in a cell-bound manner through cell division, in which the membrane functions as a reservoir for Wnt proteins ${ }^{121}$. Moreover, applying an HCS methodology to organoid culture can also be exploited to gain new insights in developmental mechanisms. These efforts aim at integrating phenotypic readouts with chemical and biological data to increase the understanding of compound action. In turn, advancements in organoid culture enable discovery of medically relevant drugs (e.g. small-molecules) potentially elucidating novel molecular mechanisms important in tissue maintenance, homeostasis and disease ${ }^{120}$.

Yet, cellular segmentation is a prerequisite for proper quantitative analysis of individual cells within populations. For complex tissues and organs a precise determination of the number of cells (nuclei) combined with fluorescence intensity values and tissue shape would be very desirable ${ }^{137}$. This automated volumetric cell segmentation can greatly benefit the high-content analysis of 3D phenotypical features in tissue and organ models and allows profiling on the effect of therapeutic molecules on tissue and organoid development or cancer progression for instance. Yet, an obvious limitation beyond the acquisition speed is the speed of analysis, which will unarguably be slower than for 2D analysis. However, the major challenge to enable routine single-cell analysis in the field of biology is to preserve software usability while implementing robust algorithms with high segmentation accuracy. Hence, advanced data analysis methods which allow multi-parametric data extraction from images of multicellular organization will enable HCS to fully flourish. 


\section{Conclusion and outlook}

The intrinsic developmental potential of stem cells and progenitor cells can be hijacked in vitro using soluble factors and 3D culture systems to instruct cells to recapitulate native development. By combining strategies such as micro-patterning, directed self-assembly and directed differentiation, tissues can be made physiologically and functionally more similar to their native analogues ${ }^{55,138-141}$. It has been demonstrated that such tissue analogues can facilitate the study of multicellular processes in vitro, including morphogenesis ${ }^{142}$, organogenesis ${ }^{91,}{ }^{138}$. Novel microfabricated platforms might enable compatibility of 3D tissue and organoids cultures with high-content screening systems and automated 3D cellular analysis, which could facilitate the discovery of novel drug compounds and study of organ development. 


\section{Aims and outline of this thesis}

As described in Chapter 1, advancing technology and increased biological insight are pushing the field of tissue engineering towards more tissue-authentic and organotypic cultures. At the same time, platforms and tools are developed that allows scaling up the formation and read-out of such tissues.

Chapter 2 Directed development and remodeling of micro-fabricated material-free tissues with complex architectures. Tissues are built in a multistep modular approach using cells as the sole component. Over time, these tissues self-reinforce themselves by ECM deposition and form a patterned pre-vascular network.

Chapter 3 outlines the fabrication of a polymeric thermoformed microwell culture plate and validation of its use as a 3D screening and monitoring platform for embryoid bodies.

In Chapter 4 an agarose-hydrogel based microwell array platform with methods of high-content analysis are employed to improve a pluripotent stem cell-based model of preimplantation embryonic development: the blastoid.

Chapter 5 includes the High-Content Screening and Analysis of embryoid bodies and blastoids to find novel compounds and pathways that direct differentiation or morphogenesis.

Chapter 6 contains my discussions based on the results described in this thesis, and a outlook on future progress in the field and its ethical concerns. Additionally, an evaluation is described on valorizing the work and its potential future applications. 


\section{References}

1. Tien, J. \& Nelson, C.M. Microstructured extracellular matrices in tissue engineering and development: an update. Annals of biomedical engineering 42, 1413-1423 (2014).

2. Zoldan, J. et al. The influence of scaffold elasticity on germ layer specification of human embryonic stem cells. Biomaterials 32, 9612-9621 (2011).

3. Bajpai, S., Kim, N.Y. \& Reinhart-King, C.A. Approaches to manipulating the dimensionality and physicochemical properties of common cellular scaffolds. International journal of molecular sciences 12, 8596-8609 (2011).

4. Bourget, J.M. et al. Human fibroblast-derived ECM as a scaffold for vascular tissue engineering. Biomaterials 33, 9205-9213 (2012).

5. Brown, B.N. \& Badylak, S.F. Extracellular matrix as an inductive scaffold for functional tissue reconstruction. Translational research : the journal of laboratory and clinical medicine 163, 268-285 (2014).

6. Butler, D.L., Goldstein, S.A. \& Guilak, F. Functional tissue engineering: the role of biomechanics. Journal of biomechanical engineering 122, 570-575 (2000).

7. Miller, J.S. et al. Rapid casting of patterned vascular networks for perfusable engineered threedimensional tissues. Nature materials 11, 768-774 (2012).

8. Nichol, J.W. \& Khademhosseini, A. Modular Tissue Engineering: Engineering Biological Tissues from the Bottom Up. Soft matter 5, 1312-1319 (2009).

9. Liu, J.S. \& Gartner, Z.J. Directing the assembly of spatially organized multicomponent tissues from the bottom up. Trends in cell biology 22, 683-691 (2012).

10. Guven, S. et al. Multiscale assembly for tissue engineering and regenerative medicine. Trends in biotechnology (2015).

11. Sekine, H. et al. In vitro fabrication of functional three-dimensional tissues with perfusable blood vessels. Nature communications 4, 1399 (2013).

12. Mironov, V. et al. Organ printing: tissue spheroids as building blocks. Biomaterials 30, $2164-2174$ (2009).

13. Tan, Y. et al. 3D printing facilitated scaffold-free tissue unit fabrication. Biofabrication 6, 024111 (2014).

14. Stevens, K.R. et al. InVERT molding for scalable control of tissue microarchitecture. Nature communications 4, 1847 (2013).

15. Leferink, A. et al. Engineered micro-objects as scaffolding elements in cellular building blocks for bottom-up tissue engineering approaches. Adv Mater 26, 2592-2599 (2014).

16. Eng, G. et al. Assembly of complex cell microenvironments using geometrically docked hydrogel shapes. Proceedings of the National Academy of Sciences of the United States of America 110, 45514556 (2013).

17. Bruzewicz, D.A., McGuigan, A.P. \& Whitesides, G.M. Fabrication of a modular tissue construct in a microfluidic chip. Lab on a chip 8, 663-671 (2008).

18. McGuigan, A.P. \& Sefton, M.V. Modular tissue engineering: fabrication of a gelatin-based construct. Journal of tissue engineering and regenerative medicine 1, 136-145 (2007).

19. Gerling, T., Wagenbauer, K.F., Neuner, A.M. \& Dietz, H. Dynamic DNA devices and assemblies formed by shape-complementary, non-base pairing 3D components. Science 347, 1446-1452 (2015).

20. Jakab, K. et al. Tissue engineering by self-assembly and bio-printing of living cells. Biofabrication 2 (2010).

21. Norotte, C., Marga, F.S., Niklason, L.E. \& Forgacs, G. Scaffold-free vascular tissue engineering using bioprinting. Biomaterials 30, 5910-5917 (2009).

22. Kang, H.W. et al. A 3D bioprinting system to produce human-scale tissue constructs with structural integrity. Nature biotechnology 34, 312-319 (2016).

23. Williams, D.F. On the mechanisms of biocompatibility. Biomaterials 29, 2941-2953 (2008).

24. Whitesides, G.M. \& Grzybowski, B. Self-assembly at all scales. Science 295, 2418-2421 (2002).

25. Jakab, K., Neagu, A., Mironov, V., Markwald, R.R. \& Forgacs, G. Engineering biological structures of prescribed shape using self-assembling multicellular systems. Proceedings of the National Academy of Sciences of the United States of America 101, 2864-2869 (2004). 
26. Steinberg, M.S. Does differential adhesion govern self-assembly processes in histogenesis? Equilibrium configurations and the emergence of a hierarchy among populations of embryonic cells. The Journal of experimental zoology 173, 395-433 (1970).

27. Foty, R.A. \& Steinberg, M.S. The differential adhesion hypothesis: a direct evaluation. Developmental biology 278, 255-263 (2005).

28. Schwartz, M.A. \& Chen, C.S. Cell biology. Deconstructing dimensionality. Science 339, 402-404 (2013).

29. Fennema, E., Rivron, N., Rouwkema, J., van Blitterswijk, C. \& de Boer, J. Spheroid culture as a tool for creating 3D complex tissues. Trends in biotechnology 31, 108-115 (2013).

30. Owaki, T., Shimizu, T., Yamato, M. \& Okano, T. Cell sheet engineering for regenerative medicine: current challenges and strategies. Biotechnology journal 9, 904-914 (2014).

31. Bergmann, O. et al. Evidence for cardiomyocyte renewal in humans. Science 324, 98-102 (2009).

32. Oltolina, F. et al. Human Cardiac Progenitor Spheroids Exhibit Enhanced Engraftment Potential. PloS one 10, e0137999 (2015).

33. DuRaine, G.D., Brown, W.E., Hu, J.C. \& Athanasiou, K.A. Emergence of scaffold-free approaches for tissue engineering musculoskeletal cartilages. Annals of biomedical engineering 43, 543-554 (2015).

34. Zhang, Z., McCaffery, J.M., Spencer, R.G. \& Francomano, C.A. Hyaline cartilage engineered by chondrocytes in pellet culture: histological, immunohistochemical and ultrastructural analysis in comparison with cartilage explants. Journal of anatomy 205, 229-237 (2004).

35. Diekman, B.O. et al. Cartilage tissue engineering using differentiated and purified induced pluripotent stem cells. Proceedings of the National Academy of Sciences of the United States of America 109, 19172-19177 (2012).

36. Wu, L., Post, J.N. \& Karperien, M. Engineering cartilage tissue by pellet coculture of chondrocytes and mesenchymal stromal cells. Methods Mol Biol 1226, 31-41 (2015).

37. Leijten, J. et al. Metabolic programming of mesenchymal stromal cells by oxygen tension directs chondrogenic cell fate. Proceedings of the National Academy of Sciences of the United States of America 111, 13954-13959 (2014).

38. Jukes, J.M. et al. Endochondral bone tissue engineering using embryonic stem cells. Proceedings of the National Academy of Sciences of the United States of America 105, 6840-6845 (2008).

39. Yang, J. et al. Tissue engineering using laminar cellular assemblies. Adv Mater 21, 3404-3409 (2009).

40. Zorlutuna, P. et al. Microfabricated biomaterials for engineering 3D tissues. Adv Mater 24, 1782-1804 (2012).

41. Baranski, J.D. et al. Geometric control of vascular networks to enhance engineered tissue integration and function. Proceedings of the National Academy of Sciences of the United States of America 110, 7586-7591 (2013).

42. Tibbitt, M.W. \& Anseth, K.S. Hydrogels as extracellular matrix mimics for 3D cell culture. Biotechnology and bioengineering 103, 655-663 (2009).

43. Khademhosseini, A. et al. Micromolding of photocrosslinkable hyaluronic acid for cell encapsulation and entrapment. Journal of biomedical materials research. Part A 79, 522-532 (2006).

44. Napolitano, A.P., Chai, P., Dean, D.M. \& Morgan, J.R. Dynamics of the self-assembly of complex cellular aggregates on micromolded nonadhesive hydrogels. Tissue engineering 13, 2087-2094 (2007).

45. Breslin, S. \& O'Driscoll, L. Three-dimensional cell culture: the missing link in drug discovery. Drug discovery today 18, 240-249 (2013).

46. Gervais, L., de Rooij, N. \& Delamarche, E. Microfluidic chips for point-of-care immunodiagnostics. Adv Mater 23, H151-176 (2011).

47. Berthier, E. \& Beebe, D.J. Gradient generation platforms: new directions for an established microfluidic technology. Lab on a chip 14, 3241-3247 (2014).

48. Stroock, A.D. \& Fischbach, C. Microfluidic culture models of tumor angiogenesis. Tissue engineering. Part A 16, 2143-2146 (2010).

49. Huh, D. et al. Reconstituting organ-level lung functions on a chip. Science 328, 1662-1668 (2010).

50. Occhetta, P. et al. High-Throughput Microfluidic Platform for 3D Cultures of Mesenchymal Stem Cells, Towards Engineering Developmental Processes. Scientific reports 5, 10288 (2015).

51. Cosson, S. \& Lutolf, M.P. Hydrogel microfluidics for the patterning of pluripotent stem cells. Scientific reports 4, 4462 (2014).

52. Dean, D.M., Napolitano, A.P., Youssef, J. \& Morgan, J.R. Rods, tori, and honeycombs: the directed selfassembly of microtissues with prescribed microscale geometries. FASEB journal : official publication of the Federation of American Societies for Experimental Biology 21, 4005-4012 (2007). 
53. Fleming, P.A. et al. Fusion of uniluminal vascular spheroids: a model for assembly of blood vessels. Developmental dynamics : an official publication of the American Association of Anatomists 239, 398406 (2010).

54. Olsen, T.R. Bioprocessing of Tissues using Cellular Spheroids. Journal of Bioprocessing \& Biotechniques 04 (2014).

55. Nelson, C.M. \& Bissell, M.J. Of extracellular matrix, scaffolds, and signaling: tissue architecture regulates development, homeostasis, and cancer. Annual review of cell and developmental biology 22, 287-309 (2006).

56. Spemann, H. \& Mangold, H. Induction of embryonic primordia by implantation of organizers from a different species. 1923. The International journal of developmental biology 45, 13-38 (2001).

57. Ogawa, M. et al. Directed differentiation of cholangiocytes from human pluripotent stem cells. Nature biotechnology 33, 853-861 (2015).

58. Ogawa, S. et al. Three-dimensional culture and cAMP signaling promote the maturation of human pluripotent stem cell-derived hepatocytes. Development 140, 3285-3296 (2013).

59. Si-Tayeb, K. et al. Highly efficient generation of human hepatocyte-like cells from induced pluripotent stem cells. Hepatology 51, 297-305 (2010).

60. Huang, S.X. et al. Efficient generation of lung and airway epithelial cells from human pluripotent stem cells. Nature biotechnology 32, 84-91 (2014).

61. Rostovskaya, M., Bredenkamp, N. \& Smith, A. Towards consistent generation of pancreatic lineage progenitors from human pluripotent stem cells. Philosophical transactions of the Royal Society of London. Series B, Biological sciences 370, 20140365 (2015).

62. Oldershaw, R.A. et al. Directed differentiation of human embryonic stem cells toward chondrocytes. Nature biotechnology 28, 1187-1194 (2010).

63. Chen, T., Wang, F., Wu, M. \& Wang, Z.Z. Development of hematopoietic stem and progenitor cells from human pluripotent stem cells. Journal of cellular biochemistry 116, 1179-1189 (2015).

64. Cohen, D.E. \& Melton, D. Turning straw into gold: directing cell fate for regenerative medicine. Nature reviews. Genetics 12, 243-252 (2011).

65. Andersson, E.R., Sandberg, R. \& Lendahl, U. Notch signaling: simplicity in design, versatility in function. Development 138, 3593-3612 (2011).

66. Moustakas, A. \& Heldin, C.H. The regulation of TGFbeta signal transduction. Development 136, 36993714 (2009).

67. Turner, N. \& Grose, R. Fibroblast growth factor signalling: from development to cancer. Nature reviews. Cancer 10, 116-129 (2010).

68. Rao, T.P. \& Kuhl, M. An updated overview on Wnt signaling pathways: a prelude for more. Circulation research 106, 1798-1806 (2010).

69. Li, W., Li, K., Wei, W. \& Ding, S. Chemical approaches to stem cell biology and therapeutics. Cell stem cell 13, 270-283 (2013).

70. de Peppo, G.M. et al. Engineering bone tissue substitutes from human induced pluripotent stem cells. Proceedings of the National Academy of Sciences of the United States of America 110, 8680-8685 (2013).

71. Fatehullah, A., Tan, S.H. \& Barker, N. Organoids as an in vitro model of human development and disease. Nature cell biology 18, 246-254 (2016).

72. Sasai, Y. Next-generation regenerative medicine: organogenesis from stem cells in 3D culture. Cell stem cell 12, 520-530 (2013).

73. Lancaster, M.A. \& Knoblich, J.A. Organogenesis in a dish: modeling development and disease using organoid technologies. Science 345, 1247125 (2014).

74. Jinek, M. et al. A programmable dual-RNA-guided DNA endonuclease in adaptive bacterial immunity. Science 337, 816-821 (2012).

75. Barker, N. et al. Identification of stem cells in small intestine and colon by marker gene Lgr5. Nature 449, 1003-1007 (2007).

76. Sato, T. et al. Single Lgr5 stem cells build crypt-villus structures in vitro without a mesenchymal niche. Nature 459, 262-265 (2009).

77. Huch, M. et al. Unlimited in vitro expansion of adult bi-potent pancreas progenitors through the Lgr5/R-spondin axis. The EMBO journal 32, 2708-2721 (2013).

78. Ren, W. et al. Single Lgr5- or Lgr6-expressing taste stem/progenitor cells generate taste bud cells ex vivo. Proceedings of the National Academy of Sciences of the United States of America 111, 1640116406 (2014). 
79. Barker, N. et al. Lgr5(+ve) stem cells drive self-renewal in the stomach and build long-lived gastric units in vitro. Cell stem cell 6, 25-36 (2010).

80. Kurmann, A.A. et al. Regeneration of Thyroid Function by Transplantation of Differentiated Pluripotent Stem Cells. Cell stem cell 17, 527-542 (2015).

81. Longmire, T.A. et al. Efficient derivation of purified lung and thyroid progenitors from embryonic stem cells. Cell stem cell 10, 398-411 (2012).

82. McCracken, K.W. et al. Modelling human development and disease in pluripotent stem-cell-derived gastric organoids. Nature 516, 400-404 (2014).

83. Takebe, T. et al. Vascularized and functional human liver from an iPSC-derived organ bud transplant. Nature 499, 481-484 (2013).

84. Greggio, C. et al. Artificial three-dimensional niches deconstruct pancreas development in vitro. Development 140, 4452-4462 (2013).

85. Boj, S.F. et al. Organoid models of human and mouse ductal pancreatic cancer. Cell 160, 324-338 (2015).

86. Spence, J.R. et al. Directed differentiation of human pluripotent stem cells into intestinal tissue in vitro. Nature 470, 105-109 (2011).

87. Noguchi, T.K. et al. Generation of stomach tissue from mouse embryonic stem cells. Nature cell biology 17, 984-993 (2015).

88. Taguchi, A. et al. Redefining the in vivo origin of metanephric nephron progenitors enables generation of complex kidney structures from pluripotent stem cells. Cell stem cell 14, 53-67 (2014).

89. Xia, Y. et al. Directed differentiation of human pluripotent cells to ureteric bud kidney progenitor-like cells. Nature cell biology 15, 1507-1515 (2013).

90. Takasato, M. et al. Kidney organoids from human iPS cells contain multiple lineages and model human nephrogenesis. Nature 526, 564-568 (2015).

91. Eiraku, M. et al. Self-organizing optic-cup morphogenesis in three-dimensional culture. Nature 472, 51-56 (2011).

92. Mariani, J. et al. Modeling human cortical development in vitro using induced pluripotent stem cells. Proceedings of the National Academy of Sciences of the United States of America 109, 12770-12775 (2012).

93. Lancaster, M.A. et al. Cerebral organoids model human brain development and microcephaly. Nature 501, 373-379 (2013).

94. Koehler, K.R. \& Hashino, E. 3D mouse embryonic stem cell culture for generating inner ear organoids. Nature protocols 9, 1229-1244 (2014).

95. Suga, H. et al. Self-formation of functional adenohypophysis in three-dimensional culture. Nature 480, 57-62 (2011).

96. Ozone, C. et al. Functional anterior pituitary generated in self-organizing culture of human embryonic stem cells. Nature communications 7, 10351 (2016).

97. Huch, M. et al. Long-term culture of genome-stable bipotent stem cells from adult human liver. Cell 160, 299-312 (2015).

98. Drost, J. et al. Sequential cancer mutations in cultured human intestinal stem cells. Nature 521, 43-47 (2015).

99. van de Wetering, M. et al. Prospective derivation of a living organoid biobank of colorectal cancer patients. Cell 161, 933-945 (2015).

100. Sugiyama, T. et al. Reconstituting pancreas development from purified progenitor cells reveals genes essential for islet differentiation. Proceedings of the National Academy of Sciences of the United States of America 110, 12691-12696 (2013).

101. Schwank, G. et al. Functional repair of CFTR by CRISPR/Cas9 in intestinal stem cell organoids of cystic fibrosis patients. Cell stem cell 13, 653-658 (2013).

102. Glowacki, J., Trepman, E. \& Folkman, J. Cell shape and phenotypic expression in chondrocytes. Proceedings of the Society for Experimental Biology and Medicine. Society for Experimental Biology and Medicine 172, 93-98 (1983).

103. Unadkat, H.V. et al. An algorithm-based topographical biomaterials library to instruct cell fate. Proceedings of the National Academy of Sciences of the United States of America 108, 16565-16570 (2011).

104. Markert, L.D. et al. Identification of distinct topographical surface microstructures favoring either undifferentiated expansion or differentiation of murine embryonic stem cells. Stem cells and development 18, 1331-1342 (2009). 
105. Rhim, J.H. et al. A High-content screen identifies compounds promoting the neuronal differentiation and the midbrain dopamine neuron specification of human neural progenitor cells. Scientific reports 5, 16237 (2015).

106. Chen, S. et al. A small molecule that directs differentiation of human ESCs into the pancreatic lineage. Nature chemical biology 5, 258-265 (2009).

107. Unadkat, H.V. et al. High content imaging in the screening of biomaterial-induced MSC behavior. Biomaterials 34, 1498-1505 (2013).

108. Lutolf, M.P., Gilbert, P.M. \& Blau, H.M. Designing materials to direct stem-cell fate. Nature 462, 433441 (2009).

109. Mei, Y. et al. Combinatorial development of biomaterials for clonal growth of human pluripotent stem cells. Nature materials 9, 768-778 (2010).

110. Boutros, M. \& Ahringer, J. The art and design of genetic screens: RNA interference. Nature reviews. Genetics 9, 554-566 (2008).

111. Scadden, D.T. Nice neighborhood: emerging concepts of the stem cell niche. Cell 157, 41-50 (2014).

112. Tape, C.J. Systems Biology Analysis of Heterocellular Signaling. Trends in biotechnology (2016).

113. Chen, S. et al. Interrogating cellular fate decisions with high-throughput arrays of multiplexed cellular communities. Nature communications 7, 10309 (2016).

114. Todhunter, M.E. et al. Programmed synthesis of three-dimensional tissues. Nature methods 12, 975981 (2015).

115. Sirenko, O. et al. High-content assays for characterizing the viability and morphology of 3D cancer spheroid cultures. Assay and drug development technologies 13, 402-414 (2015).

116. Howes, A.L., Richardson, R.D., Finlay, D. \& Vuori, K. 3-Dimensional culture systems for anti-cancer compound profiling and high-throughput screening reveal increases in EGFR inhibitor-mediated cytotoxicity compared to monolayer culture systems. PloS one 9, e108283 (2014).

117. Matano, M. et al. Modeling colorectal cancer using CRISPR-Cas9-mediated engineering of human intestinal organoids. Nature medicine 21, 256-262 (2015).

118. Joshi, P. \& Lee, M.Y. High Content Imaging ( $\mathrm{HCl})$ on Miniaturized Three-Dimensional (3D) Cell Cultures. Biosensors 5, 768-790 (2015).

119. Tung, Y.C. et al. High-throughput 3D spheroid culture and drug testing using a 384 hanging drop array. The Analyst 136, 473-478 (2011).

120. Wagner, B.K. \& Schreiber, S.L. The Power of Sophisticated Phenotypic Screening and Modern Mechanism-of-Action Methods. Cell chemical biology 23, 3-9 (2016).

121. Farin, H.F. et al. Visualization of a short-range Wnt gradient in the intestinal stem-cell niche. Nature 530, 340-343 (2016).

122. Dickinson, M.E. Multimodal imaging of mouse development: tools for the postgenomic era. Developmental dynamics : an official publication of the American Association of Anatomists 235, 2386-2400 (2006).

123. Hadjantonakis, A.K. \& Papaioannou, V.E. Dynamic in vivo imaging and cell tracking using a histone fluorescent protein fusion in mice. BMC biotechnology 4, 33 (2004).

124. Goto, T. et al. Knock-in of a histone H2B-tdTomato reporter into the Rosa26 locus allows visualization of cell nuclei in rats. Molecular reproduction and development 82, 916-917 (2015).

125. Huisken, J., Swoger, J., Del Bene, F., Wittbrodt, J. \& Stelzer, E.H. Optical sectioning deep inside live embryos by selective plane illumination microscopy. Science 305, 1007-1009 (2004).

126. Reynaud, E.G., Peychl, J., Huisken, J. \& Tomancak, P. Guide to light-sheet microscopy for adventurous biologists. Nature methods 12, 30-34 (2015).

127. Jahr, W., Schmid, B., Schmied, C., Fahrbach, F.O. \& Huisken, J. Hyperspectral light sheet microscopy. Nature communications 6, 7990 (2015).

128. Verveer, P.J. et al. High-resolution three-dimensional imaging of large specimens with light sheetbased microscopy. Nature methods 4, 311-313 (2007).

129. Pampaloni, F. et al. Tissue-culture light sheet fluorescence microscopy (TC-LSFM) allows long-term imaging of three-dimensional cell cultures under controlled conditions. Integrative biology : quantitative biosciences from nano to macro 6, 988-998 (2014).

130. Schneider, C.A., Rasband, W.S. \& Eliceiri, K.W. NIH Image to ImageJ: 25 years of image analysis. Nature methods 9, 671-675 (2012).

131. Beisken, S. et al. KNIME-CDK: Workflow-driven cheminformatics. BMC bioinformatics 14, 257 (2013).

132. Carpenter, A.E. et al. CellProfiler: image analysis software for identifying and quantifying cell phenotypes. Genome biology 7, R100 (2006). 
133. Eliceiri, K.W. et al. Biological imaging software tools. Nature methods 9, 697-710 (2012).

134. Sommer, C. \& Gerlich, D.W. Machine learning in cell biology - teaching computers to recognize phenotypes. Journal of cell science 126, 5529-5539 (2013).

135. Held, M. et al. CellCognition: time-resolved phenotype annotation in high-throughput live cell imaging. Nature methods 7, 747-754 (2010).

136. Lou, X., Kang, M., Xenopoulos, P., Munoz-Descalzo, S. \& Hadjantonakis, A.K. A rapid and efficient 2D/3D nuclear segmentation method for analysis of early mouse embryo and stem cell image data. Stem cell reports 2, 382-397 (2014).

137. Singh, S., Carpenter, A.E. \& Genovesio, A. Increasing the Content of High-Content Screening: An Overview. Journal of biomolecular screening 19, 640-650 (2014).

138. Kelm, J.M., Ittner, L.M., Born, W., Djonov, V. \& Fussenegger, M. Self-assembly of sensory neurons into ganglia-like microtissues. Journal of biotechnology 121, 86-101 (2006).

139. Rivron, N.C. et al. Tissue assembly and organization: developmental mechanisms in microfabricated tissues. Biomaterials 30, 4851-4858 (2009).

140. Tejavibulya, N., Youssef, J., Bao, B., Ferruccio, T.M. \& Morgan, J.R. Directed self-assembly of large scaffold-free multi-cellular honeycomb structures. Biofabrication 3, 034110 (2011).

141. Qi, H. et al. DNA-directed self-assembly of shape-controlled hydrogels. Nature communications 4, 2275 (2013).

142. Nelson, C.M., Vanduijn, M.M., Inman, J.L., Fletcher, D.A. \& Bissell, M.J. Tissue geometry determines sites of mammary branching morphogenesis in organotypic cultures. Science 314, 298-300 (2006). 
Chapter 1 

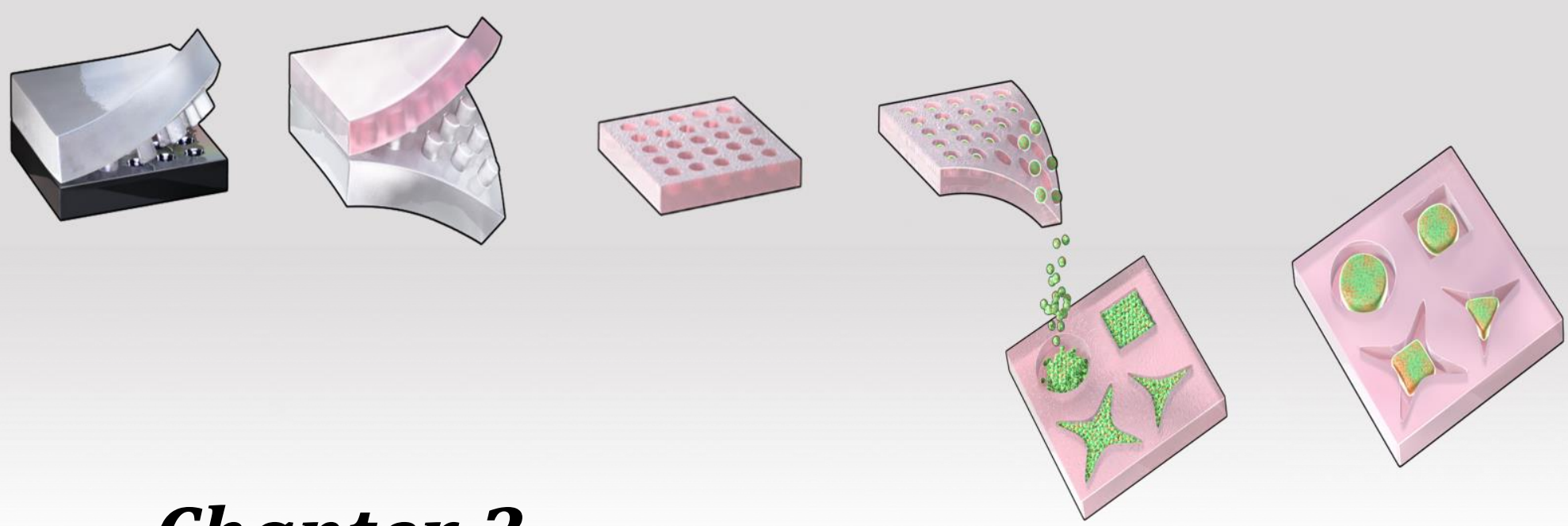

\section{Chapter 2}

\section{Directed Assembly and Development of Material- free Tissues with Complex Architectures}

Erik Vrij ${ }^{1,2}$, Jeroen Rouwkema ${ }^{2}$, Vanessa LaPointe ${ }^{1}$, Séverine Le Gac ${ }^{3}$, Albert van den Berg ${ }^{3}$, Clemens van Blitterswijk ${ }^{1,2}$, Roman Truckenmüller ${ }^{1,2}$, Nicolas Rivron ${ }^{1,2,4 *}$

1. E. Vrij, Dr. V. LaPointe, Prof. C. Van Blitterswijk, Dr. R. Truckenmüller, Dr. N. Rivron Department of Complex Tissue Regeneration, MERLN Institute for Technology-Inspired Regenerative Medicine, Maastricht University, Maastricht, The Netherlands.

2. Department of Biomechanical Engineering, MIRA Institute for Biomedical Technology and Technical Medicine, University of Twente, Enschede, the Netherlands

3. BIOS, Lab on a Chip group, MESA+ Institute for Nanotechnology, University of Twente

Drienerlolaan 5, 7522 NB, Enschede, the Netherlands

4. Hubrecht Institute for developmental biology and stem cell research, Utrecht, the Netherlands

\section{Abstract}

A long-sought goal in the field of regenerative medicine is the elaboration of scalable methods to assemble and direct the development of complex tissues for the use as models and as implants. Often, engineered tissues are constrained by supporting scaffolds and unable to naturally develop their architecture, which impairs their functionality ${ }^{1-4}$. Here we demonstrate an accessible and scalable bottom-up method to build complex three-dimensional tissues up to the centimeter scale using only cells as a building material. Tissues are fabricated through essential steps of cellular building block formation and directed differentiation on a platform compatible with high-content screening. By this method of sequential directed assembly, tissues contract, deform and change their shape stereotypically. Tissue deformation regulates vascular patterning through VEGF signaling. Tissues form a primitive vasculature, patterned by tissue deformation via VEGF signaling, and stabilize themselves through self-scaffolding. As an example, we demonstrate the formation of a tissue resembling the small bone from the inner-ear with unprecedented detail.

Keywords: directed development, bottom-up tissue engineering, cellular building blocks, tissues, high-throughput screening. 
Chapter 2 


\section{Introduction}

Tissues generated in vitro are of interest to the field of regenerative medicine to replace lost, damaged or diseased tissues, model diseases and support the development of drugs. Classically, engineered tissues are formed via a combination of cells and structurally supporting scaffolds, often made of biodegradable polymers or hydrogels. The great potential of using scaffolds lies in the freedom of geometrical design, thus yielding constructs of clinically relevant sizes and shapes. However, the biological complexity of in vitro-generated tissues is still limited. Central to this problem is that tissue development is crucially dependent on the initial three-dimensional architecture of the tissue and on its ability to freely remodel and deform ${ }^{1,5-7}$. Currently, bottom-up tissue engineering is emerging as a methodology to increase the complexity of tissues (reviewed in ${ }^{8-}$ $\left.{ }^{10}\right)$. Generally, bottom-up strategies attempt in building tissues by the self-assembly or directed assembly of cellular modules, which permits increased spatial and temporal control over the tissue architecture. ${ }^{10}$ Using such a bottom-up approach it is envisioned that the hierarchical structure of tissues is more akin to native tissues than using a classical top-down approach. Hereto, a crucial aspect is that the tissue building blocks support integration (establishment of cell junctions), structural deformation and morphogenesis of the tissue after assembly.

Bottom-up approaches often use building blocks combining cells and materials to provide bio functional cues and provisional mechanical support. A popular approach is the use of gel-like materials in which cells or aggregates of cells are embedded. For example, using 3D bio-printing ${ }^{11-14}$ or topographic molding ${ }^{15}$, tissues including cells, an extracellular matrix (ECM) and a vasculature can be formed. In an alternative approach, in this journal, we have recently shown the combination of cells with engineered cell-adherent micro-objects within a geometrically confined and cell-repellent environment that permits the formation of 3D tissues through self-assembly ${ }^{16}$. Here, the incorporation of synthetic microscale objects gives increased control over compaction and shape maintenance of the tissue compared to so far biofabricated tissues consisting solely of cells and produced via self-assembly ${ }^{17,18}$, using magnetic manipulation ${ }^{19}$ or acoustic node assembly ${ }^{20}$. However, these exogenous materials also constrain tissue remodeling and organization, and therefore tissues are restricted in naturally developing their tissue architecture.

Advancing on this study, we propose here a purely cell-based bottom-up approach that allows building stable tissue constructs with defined complex geometries. We use aggregates of cells as a living, self-scaffolding building block material for the free-form fabrication of complex 3D tissues by sequential self-assembly. To form the building blocks and resulting tissues, we developed a platform based on non-adherent hydrogel templates. This platform is used for high-throughput screening of small molecules and high-content imaging of phenotypical and gene-expression features to fine tune the physical and biological properties of the building blocks. Adequate building blocks can then be used for the directed assembly, controlled deformation and development of more complex tissues with unprecedented architectural resolution. 


\section{Results}

\section{Microfabrication of the hydrogel microwell platform}

As a platform for the controlled and uniform formation of multicellular building blocks and tissues, we fabricated a microwell array system using soft lithography methods (microwell array, Figure 1A). Microstructures were copied from a SU-8/silicon wafer via a negative replica of the same in the form of an elastomeric stamp cast from polydimethylsiloxane (PDMS) into an agarose hydrogel (Figure $1 \mathrm{~B}$ and $1 \mathrm{C}$ ). The resulting hydrogel chips including the microstructures were transferred into standard 12-well culture plates (12wp) to maximize compatibility with existing laboratory equipment. A single PDMS stamp was able to imprint arrays of several hundreds to thousands of cylindrical microwells per well of a $12 \mathrm{wp}$ and with high precision and reproducibility (Figure 1D).

$$
\text { a }
$$
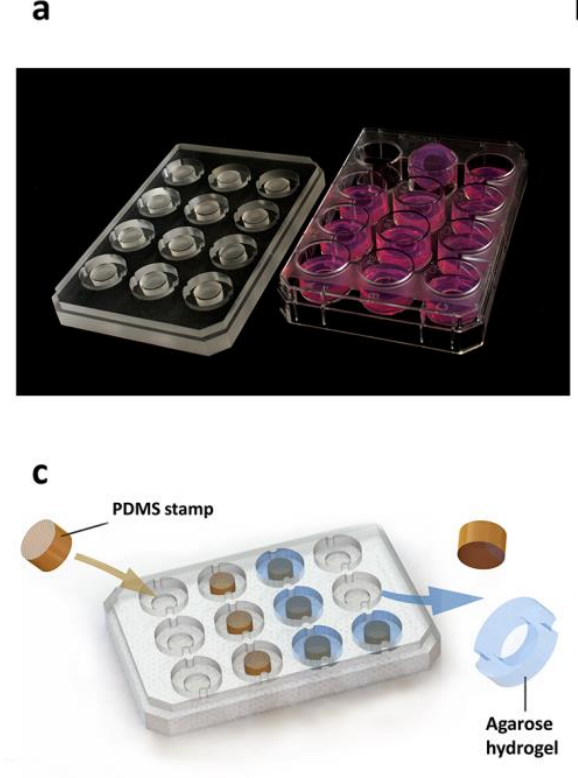

b

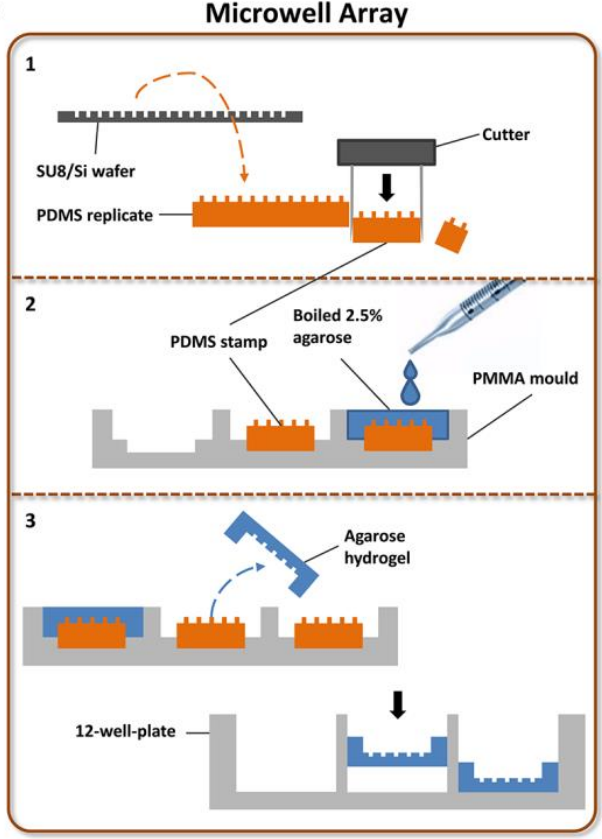

d

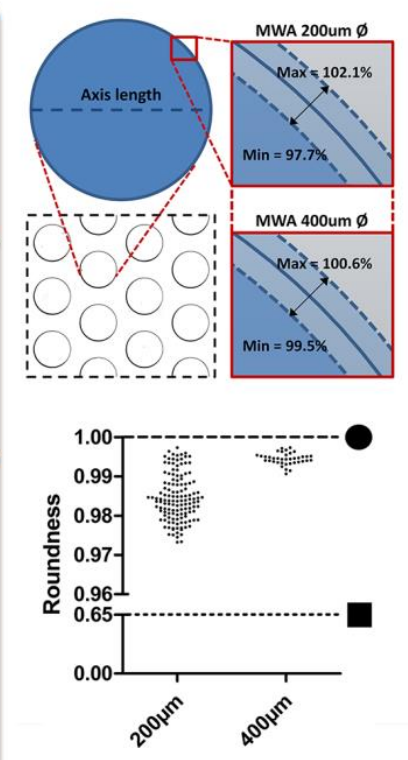

Figure 1: Microfabrication of the microwell array. (A) The microwell array was formed via replica molding in a customized 12-well plate. (B) PDMS stamps were replicated from a SU-8/Si wafer and cut to appropriate size (1). The stamps were placed in a customized 12-well plate and microstructures were replicated into agarose hydrogel (2). After solidification, agarose chips were transferred to a standard 12-well plate (3). (C) A customized 12-well plate including PDMS stamps and a microwell array plate. (D) The diameters of the microwells were $200 \mu \mathrm{m}$ and $400 \mu \mathrm{m}$. The axis lengths showed small maximal deviations. The roundness of the microwells was close to that of a circle ( $n=133$ and $n=38$ for the $200 \mu \mathrm{m}$ and $400 \mu \mathrm{m}$ diameter microwells, respectively). 


\section{Using cellular aggregates as building blocks for tissues}

As a first step for the sequential assembly of building blocks into tissues, cells were allowed to self-assemble into cellular aggregates within the agarose microwell arrays (Figure $2 \mathrm{~A}$ ). As a second step, these aggregates were used as multicellular building blocks within cavities of millimeter lengthscale hydrogel templates with defined geometry, which we termed 'geometric arrays'. Upon pooling and clustering of the aggregates onto the geometric array (projection area of $1 \mathrm{~mm}^{2}$, height of 750 $\mu \mathrm{m})$, the building blocks fused into apparent continuous tissues with defined shape within 24 hours (Figure 2A). Upon fusion and culture, the tissues remodeled, deformed and stabilized their shape (Figure 2A). Adequate culture conditions on the microwell array allowed for the fabrication of tissues with complex stable geometries including, for example, arms of $250 \mu \mathrm{m}$ in length (Figure 2B). The compaction of these tissues occurred in a controlled fashion, in contrast to bio-printing methods where tissues often distort over time. When cells were directly used to form millimeter-scale tissues, dramatic compaction occurred and tissue shape was lost ( $p<0.001$, Figure $2 \mathrm{C}$ ). This emphasized that there is a crucial necessity for fine-tuning the properties of (i) the building blocks, possibly via screening, and (ii) the sequential bottom-up approach for the maintenance of the tissue architecture.

\section{Tissue remodeling}

Upon continued culture time, circular tissues deformed isotropically, while tissues with sharp edges, for example triangular tissues, deformed anisotropically, with the greatest displacement in the corners (Figure 2D). These local contractions and deformations resulted in an internal heterogeneity in cellular density (higher cellular density in the periphery as compared to the center, Figure S1). This initial observation is consistent with the formation of patterns of endogenous mechanical stress induced within tissues by the overall geometry ${ }^{21}$. A combination of Rho/ROCK inhibitor (Y27632, $20 \mu \mathrm{M}$ ) and myosin inhibitor (blebbistatin, $50 \mu \mathrm{M})$, stopped tissues from contracting and shifting shape: trifolium-shaped tissues contracted $9 \%$ when treated with a combination of Y 27632 and blebbistatin, as compared to $39 \%$ in the untreated control (Figure S2). This demonstrates that the deformation of tissues is an active biological process driven by the actinmyosin complex. Taking the anisotropy of deformation into account, we designed geometric arrays guiding the tissue to deform toward a desired final geometry such as a square or triangle (Figure 2D). A compensated square geometry led to a tissue that deformed and remodeled into a tissue which overlaps more with a perfect square (97\%) than an uncompensated, real square geometry $(94 \%$, Figure $2 \mathrm{E}$, for comparison; a perfect circle overlaps $91 \%$ with a perfect square). Interestingly, tissue remodeling can further be manipulated by introducing a shaped constraint inside the geometric array (Figure 2F). The shape and the direction of these inner constraints modulated the outer shape of the tissue, thus adding a supplementary layer of control over deformation. Together, this showed that outer and inner geometries can be designed as such to guide the deformation and form tissues with precise geometries. 
a

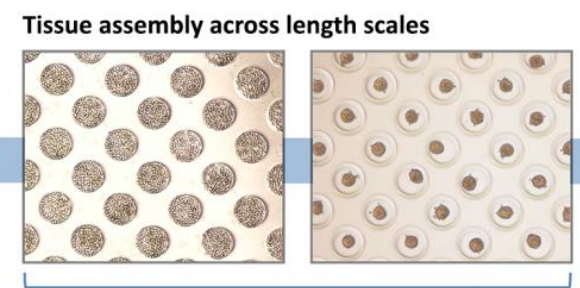

Spheroids $[\approx 100 \mu \mathrm{m}]$ on the microwell array

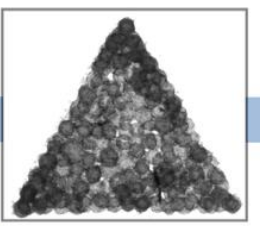

Tissues $[\approx 1000 \mu \mathrm{m}]$ on the geometric array

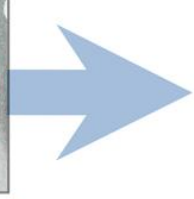

Assembly of shapes

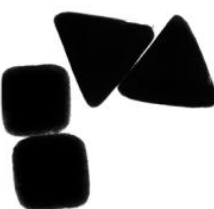

b

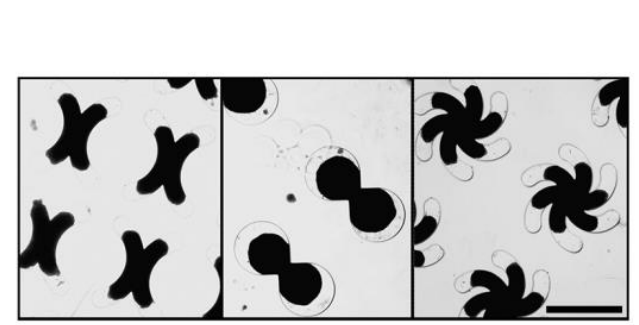

C
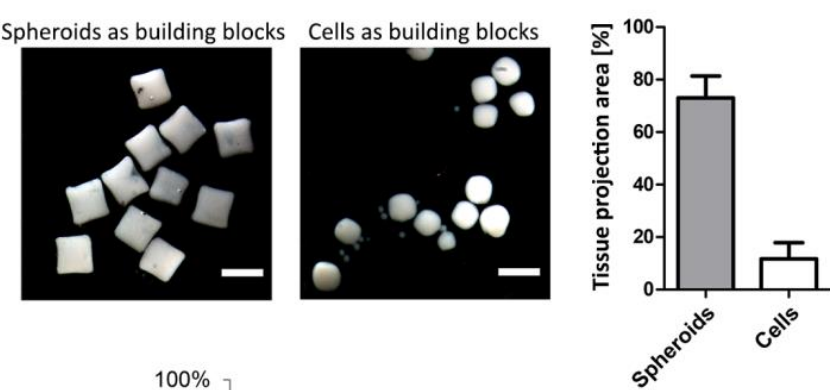

d

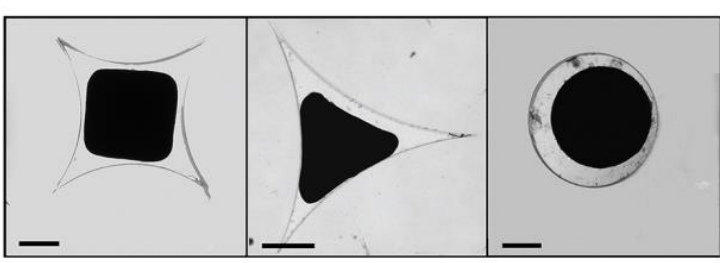

e

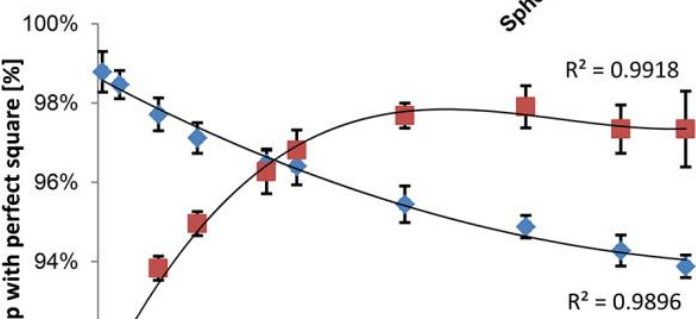

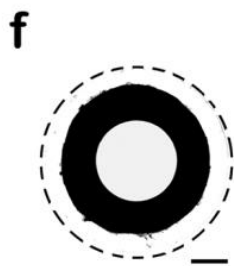

Circl. $=0.82$

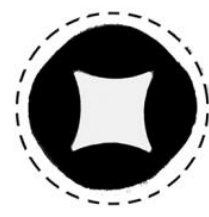

Circl. $=0.69$

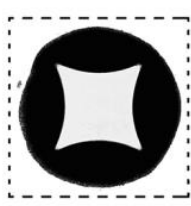

Circl. $\mathbf{=} \mathbf{0 . 8 0}$ 
tissue shape. In contrast, an inner star-shaped constraint changed the outer shape of the circular tissue. In square-shaped tissues, a star-shaped inner constraint better maintained an outer square tissue shape when the constraint was rotated by 45 degree. Dashed lines represent the outlines of the geometric wells. Scale bar represents $200 \mu \mathrm{m}$.

The phenotype and function of the assembled tissue are defined by the properties of its building blocks, such as aggregate size, cell type(s), culture (differentiation) medium and culture time. Finetuning these conditions is crucial and allows to modulate the properties of the assemble tissue. For example, we found that the optimal culture time for aggregates from human mesenchymal stromal cells (hMSCs) to fuse into a continuous tissue while maintaining a final precise square or triangular shape was 5 days (Figure 2D and S3). To modulate the size of building blocks, we formed cellular aggregates of hMSCs with controlled diameters ranging from 35 to $150 \mu \mathrm{m}$, depending on the average number of cells per microwell and on the size of the microwells (Figure 4A). Upon aggregation, the diameter of the cellular aggregates increased, decreased or remained stable over time depending on the culture condition (differentiation media, Figure 4A). For seven different cell types, we classified the stereotypical behaviors into three categories - as 'compacting', 'growing' or 'stable' aggregates - based on their projection area and circularity (Figure S4 A, B and C, respectively). The time of culture on the microwell array along with the cell type dependent stereotypical behavior strongly influenced and allowed to control the remodeling of the tissue when these aggregates were used as building blocks. As an example, 'compacting' spheroids of hMSCs cultured for a longer period of time on the microwell array permitted an increased maintenance of the geometric shape (Figure 4A).

\section{Hydrogel microwell screening platform}

Differentiation media, such as used earlier to steer the cell fate of hMSC aggregates towards the osteogenic, chondrogenic or adipogenic lineage, contain soluble factors that act on specific genetic circuits within the cells. For example, the small molecule steroid dexamethasone is used to drive cell fate towards the osteogenic lineage ${ }^{22}$. In that regard, functional screening for small chemicals is a valuable method to define conditions that steer stem cell fate and direct the differentiation toward specific cell types ${ }^{23,24}$. In order to identify novel chemicals modulating the properties of the cellular building blocks, we adapted the microwell array platform to a standard 96-well plate (96wp) format that allows the high-throughput screening (HTS) of small molecules (screening array, Figure 3 ). Within each well of a 96wp there are 580 microwells (replicates) reproducibly fabricated with high spatial resolution (Figure 3D). This conferred compatibility with standard equipment for highcontent screening $(\mathrm{HCS})$ and imaging $(\mathrm{HCl})$. 
a

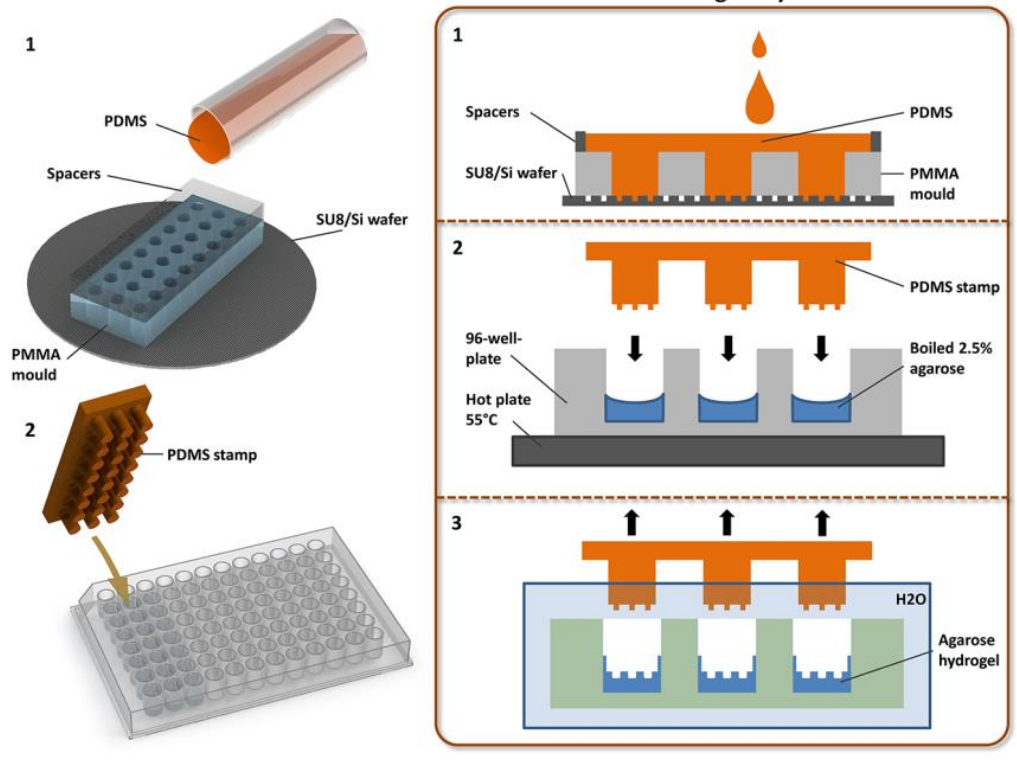

C

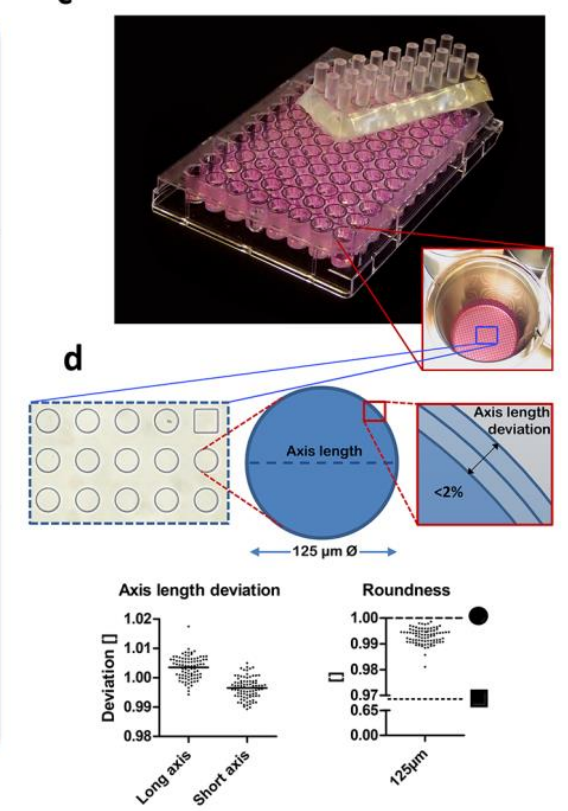

Figure 3: Microfabrication of the screening array. (A) The screening array was formed via replica molding into a hydrogel directly within a conventional 96wp. (B) An elastomeric stamp was cast into PDMS onto an assembly of a PMMA mold, a surrounding spacer and a patterned Si/SU-8 wafer, and cured (1). Microstructures were replicated into a hydrogel, within a 96wp (2). Upon hydrogel solidification, the stamp was released by submerging the assembly in water (3). (C) A PDMS stamp and a screening array plate. Each well of the $96 \mathrm{wp}$ contains 580 microwells. (D) The microwells have a diameter of $125 \mu \mathrm{m}$ with a deviation of less than $2 \%$ as measured along two orthogonal (long and short) axes. The roundness of the microwells is close to that of a circle $(n=86)$.

\section{Automated read-out of fluorescent transcriptional reporters}

Using this platform, we formed aggregates of mouse embryonic stem cells (mESCs). These mESC aggregates, named embryoid bodies (EBs), formed reproducibly within 24 hours of culture (Figure S5A). Phenotypical parameters, such as projection area and circularity, were acquired directly on the screening array using bright-field and fluorescence microscopy (Figure S5B). The variability of the size (projection area) of the EBs was low (Figure S5D). Circularity never deviated more than $2 \%$ (Figure S5E). We validated the platform for $\mathrm{HCl}$, using a $\mathrm{mESC}$ line combining fluorescent reporters for the promotor activity of the transcription factor Nanog (Nanog-RFP), a gene associated with selfrenewal and pluripotency, and a single allele knock-in for Sox17 (Sox17-GFP), a gene involved in the first differentiation steps into primitive endoderm (Figure S6) ${ }^{25}$. The promoter activities of Nanog and Sox 17 were modulated by administering small molecules with previously assessed activities on these genes ${ }^{26-28}$, monitored by automated fluorescence microscopy and quantified. Consistent with studies on the maintenance of pluripotency in mESCs, we show that Nanog-RFP fluorescent intensity was maintained in 2i/LIF (GSK3 $\beta$ and MEK/ERK inhibitor, supplemented with leukemia inhibitory factor) and it reproducibly decreased upon exposure to a combination of retinoic acid (RA) and Activin A within 48 hours (Figure 4B). Alternatively, and consistent with studies on the induction of primitive endoderm, Sox17-GFP fluorescent intensity remained stable upon exposure with 2i/LIF and increased upon stimulation with RA and Activin A (Figure 4B). Together, these results highlight the 
sensitivity of the quantification of fluorescent reporter activities as a read-out for the directed differentiation of cellular building blocks.

\section{High-Content Screening of a small molecule kinase inhibitor library}

Then, to demonstrate that a specific building block phenotype can be directed and monitored, we used a kinase inhibitor library to identify kinases involved in the directed differentiation of EBs towards primitive endoderm (Figure 4C). Differentiation was assessed by H2B-GFP reporter activity from a mESC line with a PDGFR $\alpha^{\text {H2B-GFP/+ }}$ knock-in allele (Figure S6) ${ }^{29}$. As PDGFR $\alpha$ is a plasma membrane-bound receptor, the nuclear translocation of the GFP protein increased the quality of the fluorescent read-out. Differentiation was quantified as the ratio of EBs positive for H2B-GFP compared to the total number of EBs (Figure 4D shows representative images of four compounds with decreasing H2B-GFP expression), and the projection area was averaged over approximately 300 EBs per condition. Consistent with other reports and our previous observation using the Sox17 reporter, the Mek1/Mek2 inhibitors (PD325901 and U0126) were found to inhibit PDGFR $\alpha$ expression. The screen revealed that the protein kinase A (PKA) inhibitor H-89, an ATP-competitive PKA phosphorylation inhibitor, strongly inhibited H2B-GFP expression ( 0 out of 320 EBs were positive for H2B-GFP expression). Tyrphostin 47 and 51, both epidermal growth factor (EGF) receptor kinase inhibitors, and $\mathrm{HBDDE}$, a protein kinase $\mathrm{C}$ inhibitor, were found to increase the yield of H2B-GFP positive EBs $(3.5,1.5$ and 2.3 standard deviations above the mean of neutral controls, respectively). These results show that small molecule screens can be used to find novel regulators for directed differentiation of 3D tissue building blocks. 
a
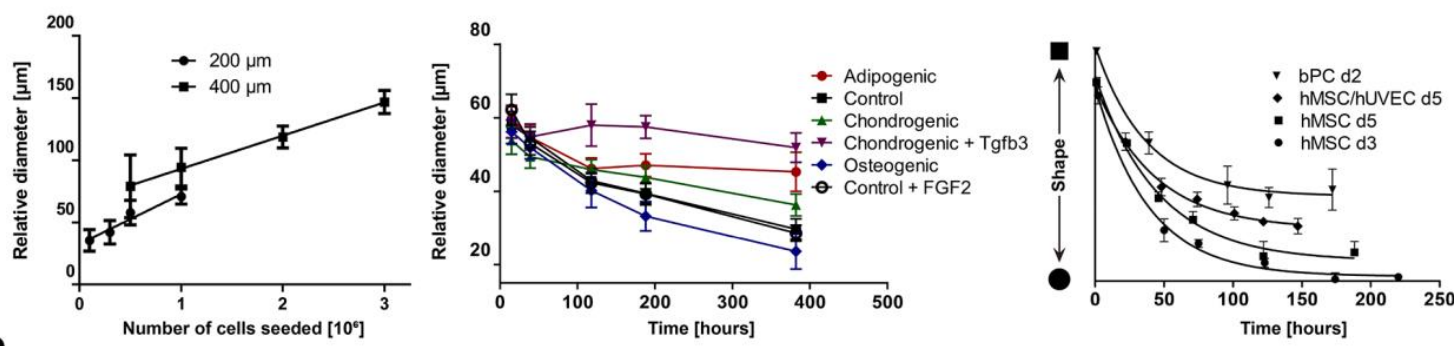

b
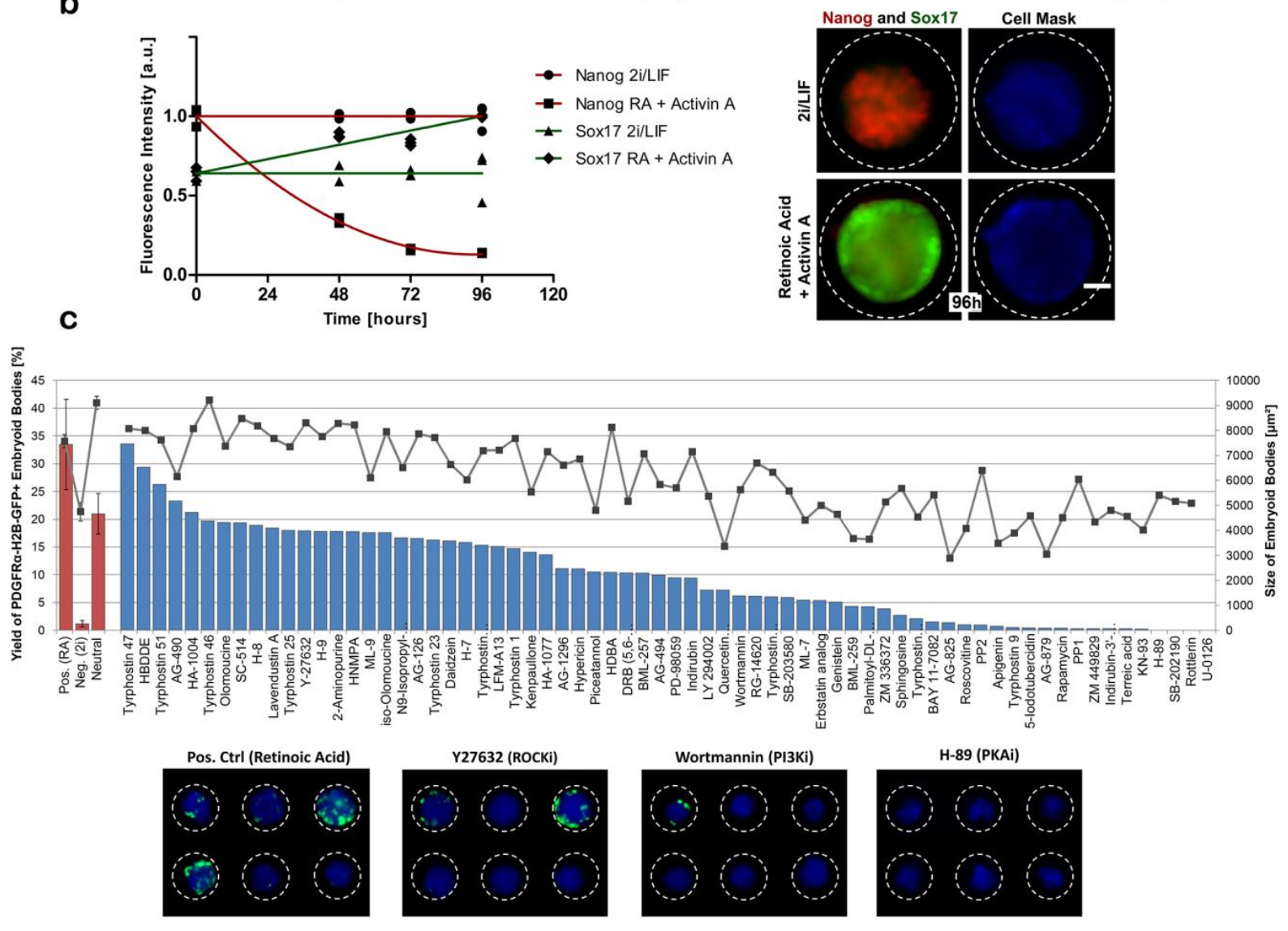

Wortmannin (PI3Ki)

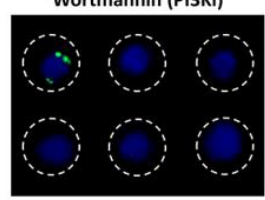

H-89 (PKAi)

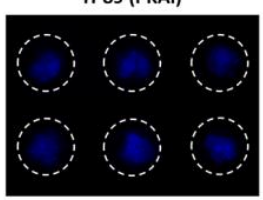

Figure 4. Modulation of building blocks. (A) Tuning the properties of building blocks: (1) The relative diameter by adjusting cell number in combination with microwell size, (2) cellular differentiation by defined media, and (3) cell type and culture time define the plasticity of resulting tissue. (B) Monitoring differentiation status of building blocks on-chip by fluorescent read-out. Left: the time course of the fluorescent expression of NanogRFP and Sox17-GFP reporters in mESCs was recorded upon modulation using small molecules. The combination of the small molecules PD0325901 and CHIR99021 with the protein leukemia inhibitory factor (2i/LIF) maintained the high expression of Nanog-RFP and the low expression of Sox17-GFP over 96 hours, whereas a combination of retinoic acid and Activin A induced a decrease of Nanog-RFP and an increase in Sox17-GFP. Fluorescence intensity of $15 \mathrm{EBs}$ per well ( $n=3$ wells) was measured and normalized to overall highest and lowest intensity for RFP and GFP, respectively. Right: fluorescence images of representative EBs treated with $2 \mathrm{i} /$ LIF or RA for 96 hours. Scale bar represents $25 \mu \mathrm{m}$. (C) Using HCS to find chemicals that tune building blocks. A screening using a kinase inhibitor library and a mESC line comprising a PdgfraH2B-GFP/+ reporter identified regulators of the primitive endoderm marker PDGFR . Tyrphostin 47 (EGFr inhibitor, 33\% of EBs positive for H2B-GFP) and U0126 (Mek1/Mek2 inhibitor, 0\% of EBs positive for H2B-GFP) were activators and inhibitors of PDGFR $\alpha$ expression, respectively. The percentage of EBs including GFP+ cells (blue bars) was measured and determined according to approximately 130 EBs per condition. The projection area of EBs was measured as an approximation for their volume and varied between 2500 and $9500 \mu \mathrm{m} 2$. The negative control, a combination of PD325901 and CHIR-99021 [28], led to an average of $1.2 \%$ of EBs that was positive for H2B-GFP expression, $n=6)$. Representative images of six EBs per condition; positive control (RA) and three kinase inhibitors. Scale bar represents $100 \mu \mathrm{m}$. 


\section{Trade-off between tissue remodeling potential and stability}

Tuning the physical and biological properties of the building blocks by appropriate culture time and medium components appeared a critical step for the formation of stable geometric tissue constructs of millimeter-scale. This balance between remodeling and stability is partly dependent on the balance of cell-cell and cell-matrix interactions ${ }^{30}$. Similar to native tissues in which the ECM acts as an endogenous scaffold that embeds the cells, these tissue constructs should reinforce themselves over prolonged culture time by the secretion of their own ECM. To prove this, shaped tissues from bovine chondrocytes were cultured for 3 weeks and treated with transforming growth factor (TGF) $\beta 3$, which yielded the formation of a continuous Safranin-O positive ECM (Figure 5A and Figure S7). This result indicates that tissue constructs have the capacity to remodel and stabilize themselves by self-scaffolding, thereby mimicking native tissue architecture. Important in this conjunction is that tissues of increased size should have the capacity to form a vascularized network to overcome the limitations of nutrient and oxygen supply and waste disposal, which might lead to a necrotic core. This is especially important for tissues with high metabolic activity or tissues that develop a less permeable outer shell (e.g. ECM).We and others have previously shown that vascular networks can self-organize in vitro ${ }^{31,32}$, further develop using morphogens (e.g. Sonic Hedgehog) and anastomose with the host's vasculature upon implantation ${ }^{31,33,34}$. Here, we formed building blocks using a combination of human umbilical vein endothelial cells (hUVECs, positive for CD31) and hMSCs. Upon fusion into a tissue, the hUVECs sprouted and self-organized to form a pre-vascular network ranging over several hundred micrometers, thus spanning over several building blocks (Figure 5B and Figure S8A). Moreover, in the presence of the morphogen Sonic hedgehog, the endothelial cells formed lumens resembling blood vessels (Figure 5C and S8B). Of note, the formation of a necrotic core occurs in certain conditions but can be circumvented by optimizing the culture conditions according to the cell type. The tissues, formed with hMSCS and HUVEC or with chondrocytes, do not exceed $300 \mu \mathrm{m}$ in, at least, one direction, and showed no necrotic core (Figure S9). Importantly, tissue building blocks retain a degree of plasticity which permits the recapitulation of differentiation and morphogenetic processes, thus allowing novel insights into developmental mechanisms acting at the tissue scale ${ }^{1}$. If cultured for longer time on the microwell array, building blocks can lose their plasticity, which affects the flow of the cells upon fusion and the homogeneity of the final tissue (Figure S3). Thus, the fine-tuning of the physical and biological properties of the building blocks permits a balance between the desired remodeling required for morphogenesis and the stability of the final geometry. 


\section{Assembling tissues of clinically relevant size}

For therapeutic use, it is highly favored that tissue grafts mimic the native tissue architecture. Here we tested the possibility to assemble tissues that mimic the anatomical shape of the human stapes, which is a small bone in the middle ear, and the smallest bone found in the human body (approximately 2 by $3 \mathrm{~mm}$ in size). Upon assembly of cells and successively of building blocks from these cells, structurally stable tissues were formed with a size and 3D architecture resembling the stapes (Figure 5D and E). The in vitro-fabricated stapes tissues consisted of human cellular material only and were of unprecedented architectural resolution, thus demonstrating the potential of forming precisely defined shapes using cellular building ocks (Figure S10). The surface and inner properties of the tissues, as investigated using scanning electron microscopy, showed a smooth outer surface and an inner fibrillar structure typical of the extra-cellular matrix (Figure S11).

To demonstrate the flexibility to further extend the sequential assembly of tissue constructs, circular shaped tissue modules were assembled into cylindrical tissues (Figure $5 \mathrm{~F}$ and $\mathrm{G}$ ), and square and triangular shaped tissue modules were assembled into a tissue sheet, in a puzzle-like manner (Figure $5 \mathrm{H}$ ). The tissue modules were manually placed within agarose hydrogel confinements and fused within 24 hours to form tissues of centimeter length-scale which were easily handled using surgical instruments, a proof of their self-reinforcing and -supporting character. We propose these examples of confined self-assembly as a potential means to fabricate tissues with clinical relevance. 

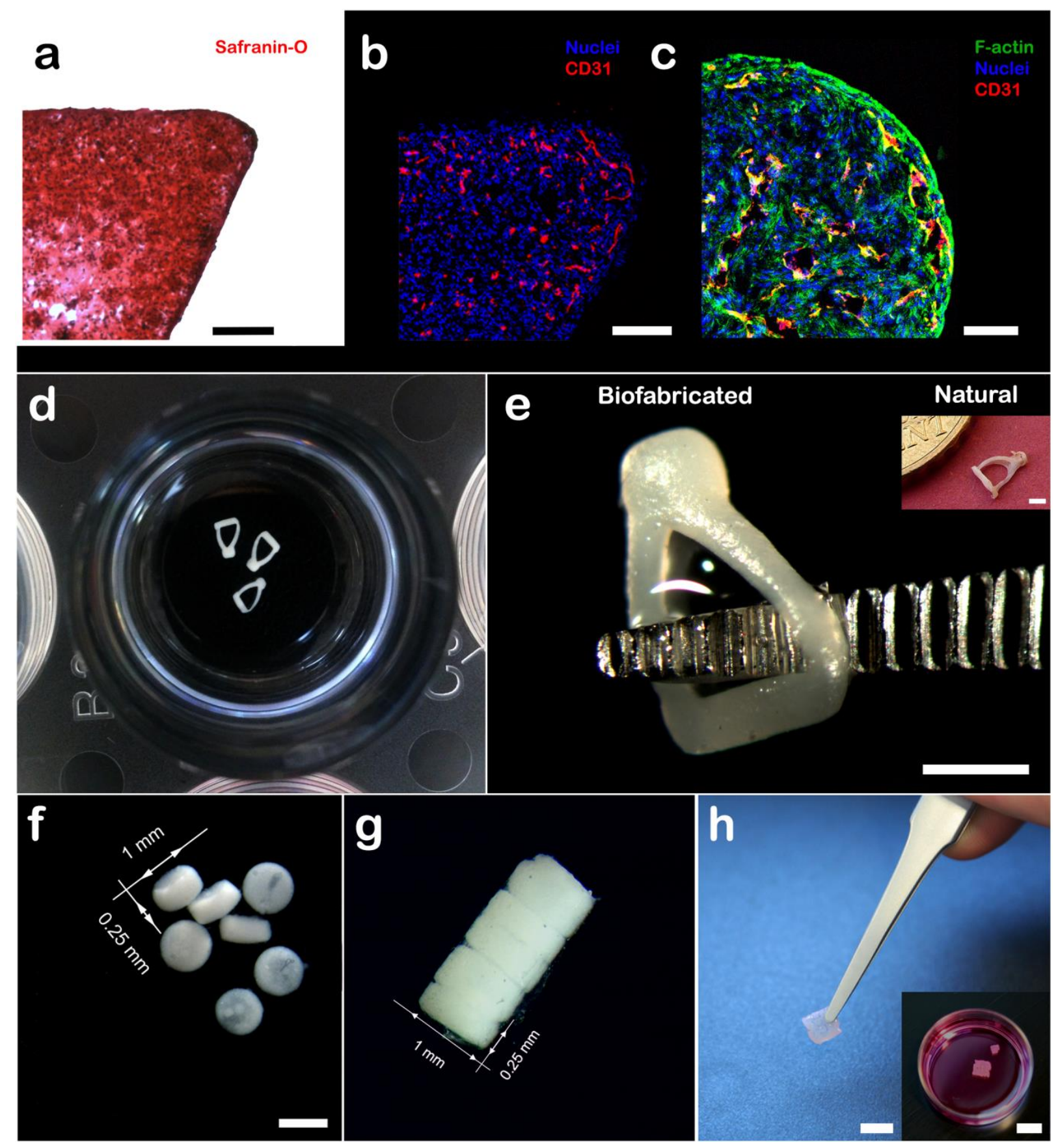

Figure 5. Tissue differentiation, formation and assembly. (A) Self-scaffolding of tissues. Building blocks formed using bovine primary chondrocytes fused into a tissue that produced an extensive ECM (positive for Safranin-O staining) within 21 days. Scale bar represents $200 \mu \mathrm{m}$. (B) Pattern formation of primitive vasculature within tissues. Building blocks formed by mixing human mesenchymal stem cells and human umbilical vein endothelial cells fused into a continuous tissue, in which endothelial cells (CD31+) sprouted to form a primitive vasculature within 150 hours. Scale bar represents $200 \mu \mathrm{m}$. (C) Vasculogenic patterns formed by adding the morphogen Sonic Hedgehog (spherical tissue of $400 \mu \mathrm{m}$ in diameter cultured for 288 hours). Scale bars represent $50 \mu \mathrm{m}$. (D-E) Tissue formed resembling the stapes, a middle-ear bone, with a clinically relevant size and three-dimensional shape, as compared to the natural tissue (image of human stapes by Bruno Welleschik [29]).Scale bars represent $1 \mathrm{~mm}$. (F-G) Millimeter-sized circular microtissues were assembled and spontaneously fused to form a cylindrical structure. Scale bar represents $1 \mathrm{~mm}$. (H) The assembly of square and triangular shaped tissues led, upon fusion, to centimeter-scale, easy-to-handle tissues. Scale bar represents $1 \mathrm{~cm}$. 


\section{Microfabricated tissues as a tool to study novel mechanisms involved in multicellular development}

Physical forces, both endogenous and external, play key roles in the organization of developing tissues ${ }^{35}$, including tissue repair such as wound healing. The actin-myosin networks within cells generate forces which are transmitted between cells through adhesive complexes, which together drive morphogenesis ${ }^{36}$. How the local generation of forces by cells is integrated over tissue-scale distances of thousands of cells, however, remains largely unknown. Here, we used engineered tissues based on cell-only building blocks to elucidate clues about the role of contraction and deformation in modulating the formation of vascular patterns. Engineered tissue constructs were formed by assembling spheroidal multicellular building blocks consisting of hMSC (92\%), which can autonomously generate forces ${ }^{37}$ and produce the angiogenic mitogen vascular endothelial growth factor (VEGF) ${ }^{38,39}$, and hUVEC (8\%), which are capable of forming a 3D prevascular network in vitro. Such an engineered system is a relevant model for early vascularization ${ }^{32}$.

\section{Tissue contraction modulates vascular patterning}

Multicellular building blocks consisting of hMSC with $8 \%$ hUVEC were pooled into geometric arrays of various shapes (triangle, square and circle) where they fused together within 24 hours, compacted and deformed shape according to their outer template geometry (Figure 6A). Circular tissues remodeled isotropically, whereas square-shaped tissues remodeled anisotropically with tissue corners displacing more rapidly than the respective sides (Figure 6A). Template geometries that were designed to compensate for this shape shifting allowed tissues to progressively remodel towards predicted square and triangle shapes (Figure 2E, Figure 6A). Importantly, compensated square tissues demonstrated more extensive remodeling of their corners than normal square tissues (Figure 6C), which can be exploited to study the effect of deformation on vascularization. Indeed, after immunostaining for vascular structures (CD31+ cells), these revealed a gradient increasing in density from the tissue center to the corner of compensated square geometries. A similar effect accounted for the distribution of cell nuclei. Interestingly, after normalizing for the nuclei distribution, the density of vascular structures (CD31+) was retained and showed a 2.5 fold increase from center to corner in deformed tissues (Figure 6D). As such, deformed tissues showed preferential formation of vascular structures (CD31+ cells) in regions of high contraction. Moreover, when tissue contraction was impaired as described earlier (see Figure S1), preferred local formation of CD31+ structures in the corners was severely attenuated to only a 1.4 fold increase from tissue center to corner (Figure 6D). This demonstrates that the increase in density of vascular structures can only be partially attributed to the increase in tissue density and thus suggests a more complex role for tissue contraction in angiogenesis. 
a

$24 \mathrm{~h}$
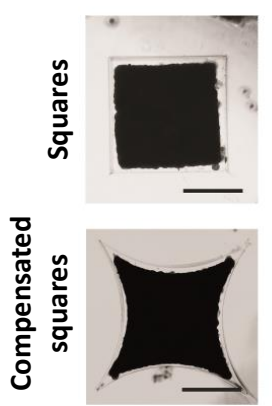

C

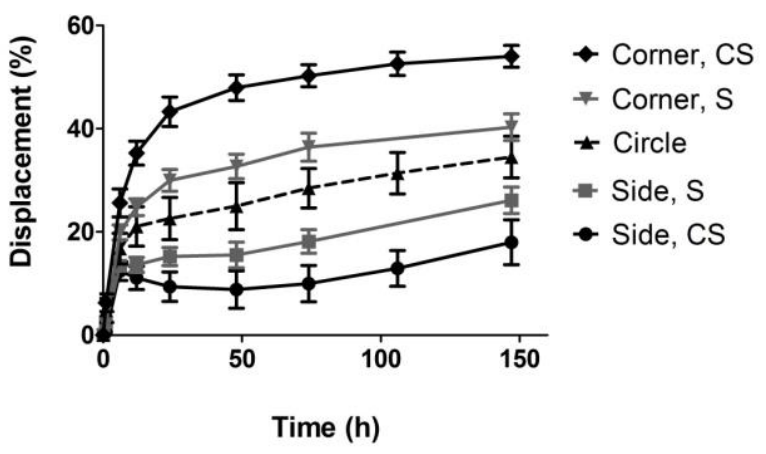

$48 \mathrm{~h}$
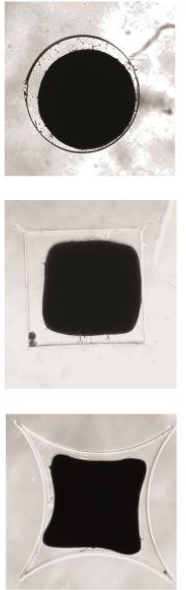

$72 \mathrm{~h}$
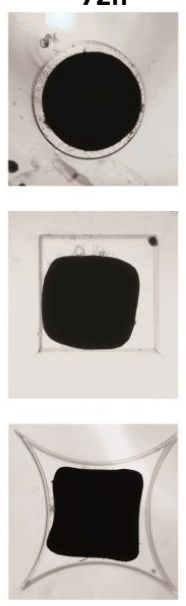

$96 \mathrm{~h}$
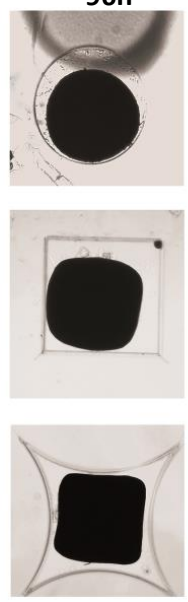
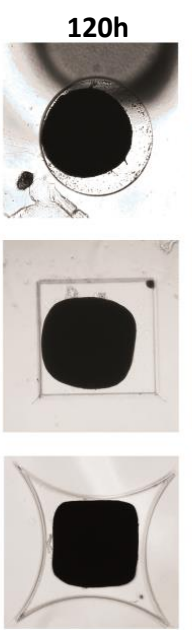
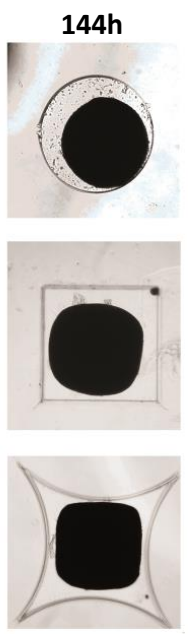

b

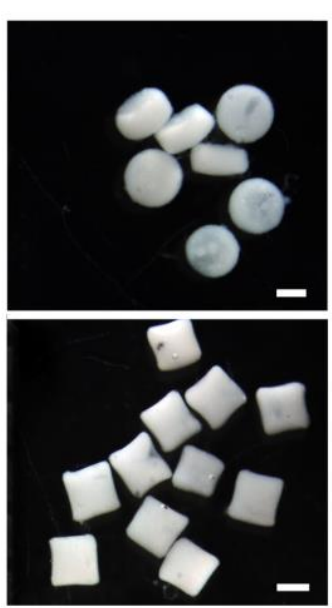

d
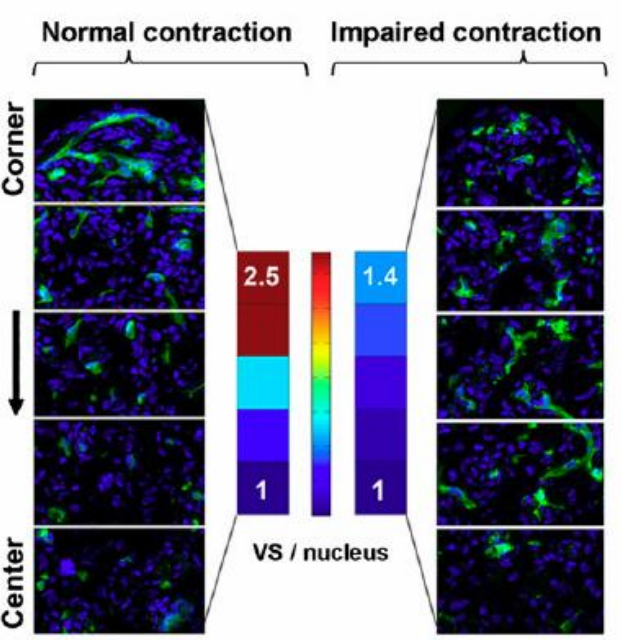

Figure 6: Anisotropic deformation leads to a heterogeneous pattern of angiogenesis. (A) Upon culture in geometrical arrays, tissues compacted and deformed according to their external geometries and stabilized their shapes after 150 hours. Circular tissues remodeled isotropically while square tissues remodeled anisotropically. (B) Tissues after 72 hours of culture. (C) Displacement of tissue borders was measured normalized to the initial distance between the center and the periphery using bright field images (displacement $=100-\%$ of the original distance, $n=5$ ). (D) Angiogenesis, depicted by immuno-stained CD31+ structures, showed a gradient from tissue center to corner. This effect was reduced when tissue contraction was impaired using a combination of blebbistatin and y27932. Figure 6D courtesy of Nicolas Rivron and adapted from ${ }^{1}$. Scale bars represent $500 \mu \mathrm{m}$.

\section{Tissue deformation spatially regulates VEGF signaling}

VEGF signaling plays a key role in regulating the morphogenic events during angiogenesis ${ }^{40}$. It is believed that local gradients of VEGF, amongst other signaling components such as Notch, permit patterning the vascular network ${ }^{41,42}$. Immunohistochemical fluorescent stainings in tissues of normal and impaired contraction revealed a correlation between tissue contraction (high in corners, low in centre) and the formation of a molecular gradient of VEGF (Figure 7A and B). VEGF 
immunofluorescence staining intensity was found twofold higher in regions of high deformation (Figure 7B). To assess whether such increase in staining intensity corresponds to higher concentrations of VEGF protein, a concentration series of Deferoxamine (DFO) was examined (Figure $\mathrm{S} 12)$. DFO is an iron chelator compound known to upregulate the expression of angiogenic factors such as VEGF by mimicking hypoxia ${ }^{43}$. A positive linear correlation between VEGF concentration and VEGF immunofluorescence intensity could be derived for the concentration ranges that were observed using ELISA protein measurements (Figure S12). This implies that the degree of VEGF immunofluorescence intensity scales accordingly with the amount of VEGF protein present in tissue samples. Congruent with these observations, the total amount of VEGF production was higher in contracting tissues (Figure 7C).

\section{Matrix-bound isoforms VEGF-165 and -189 regulate local angiogenesis}

Alternative exon splicing gives rise to various isoforms of VEGF which orchestrate angiogenesis 44. VEGF isoforms show differential binding to ECM and cellular membranes due to the presence or absence of heparin-binding domains, which can modulate their localization within the extracellular domain and enables the formation of molecular gradients ${ }^{45}$. Hence, the shortets isoform VEGF-121 is not a heparin-binding protein and thus freely diffusible, while VEGF-189 is mostly immobilized at the cell surface and in matrices. Exposure of endothelial cells to matrix-bound VEGF elicits distinct and extended activation of VEGFR2 ${ }^{46,47}$. In normal contracting cell pellets it was observed that VEGF-165 and VEGF-189 showed greater differential mRNA expression than in tissues in which contraction was impaired (more than 2.5 fold higher expression, $p<0.01, n=3$, Figure 7D). Conversely, the expression of VEGF-121 and VEGF-145 remained unchanged. Interestingly, expression of VEGF-121 was most abundant in absolut terms for tissues both impaired and normally contracting (Figure S13). Together with the finding that contraction particularly occurres during the first days of culture (highest strain), this suggests that signaling from immobilized VEGF has a dominant contribution in spatial patterning of angiogenesis in contracting tissue constructs. 
a

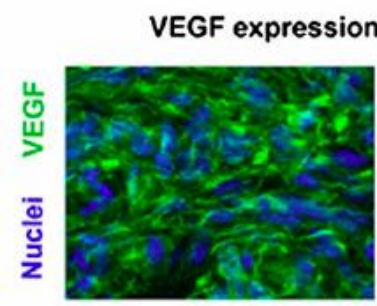

Normal contraction

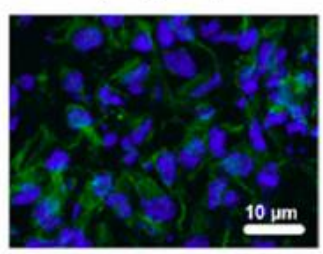

Impaired contraction

b
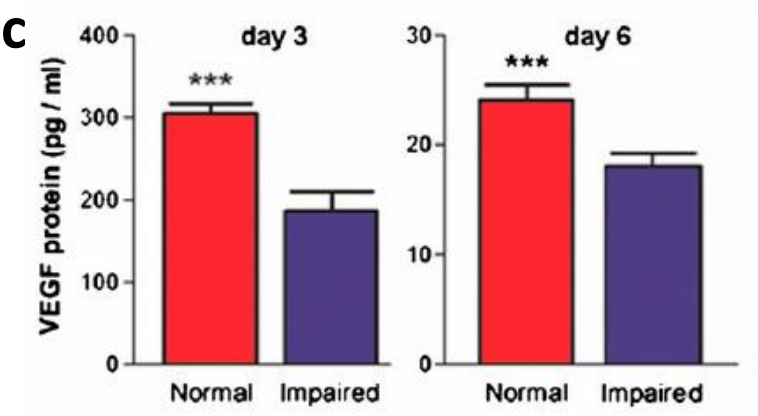

d
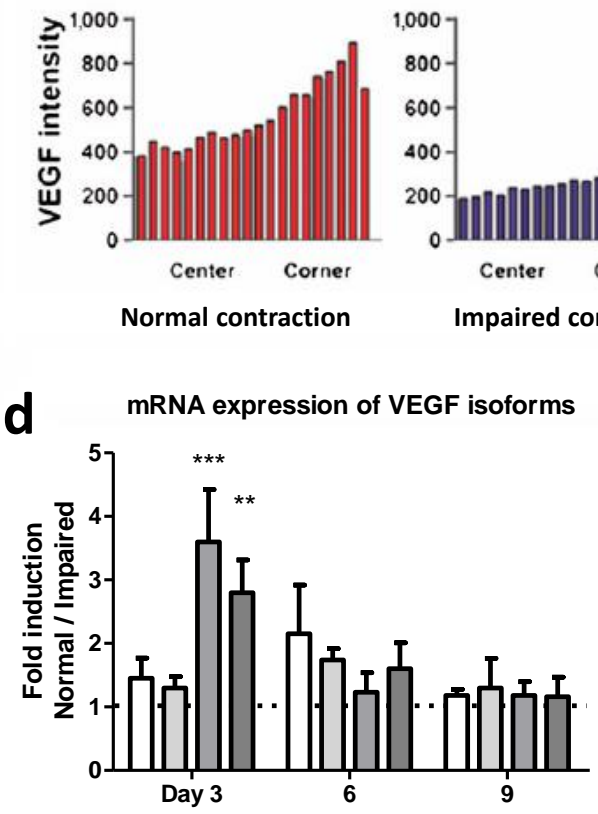

Normal contraction

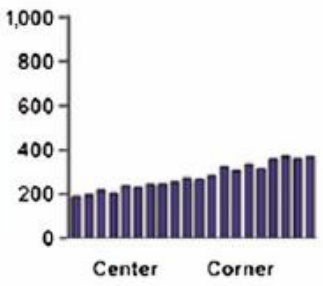

Impaired contraction

d

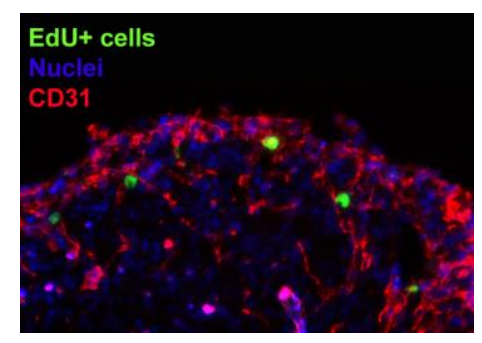

e

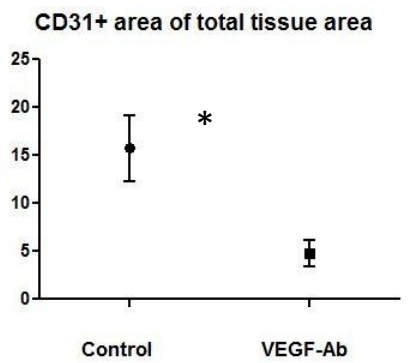

f

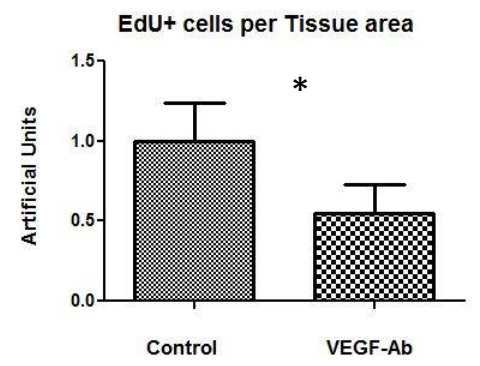

Figure 7: Tissue deformation regulates VEGF signaling. (A) Illustrative images of VEGF immunofluorescence intensity. (B) Graded intensity of VEGF immunofluorescence showed a steeper gradient in tissues from the center to the corner upon normal contraction compared to impaired contraction. Figure $7 A, B$ and $C$ courtesy of Nicolas Rivron and adapted from ${ }^{1}$. (C) The total production of VEGF-A ligands was higher in cell pellets undergoing normal contraction than when contraction was impaired $(p<0.001)$. (D) Expression of mRNA product for VEGF-121, 145, 169 and 189 proteins in contracted tissue pellets normalized to tissues that were impaired for contraction. Impaired contraction specifically affected the expression of VEGF isoforms 165 and 189 (ECM-binding isoforms, $* * *$ and $* *$ denote $\mathrm{p}<0.001$ and $\mathrm{p}<0.01$ respectively, $\mathrm{n}=3$, ANOVA with Bonferroni post-test). Expression levels of isoforms 121 and 145 (non-ECM-binding isoforms) remained unchanged. (E) EdU+ cells were mostly found in the tissue periphery and mostly overlapped with the endothelial marker CD31. (F) Scavenging of endogenous VEGF in cell pellets induced a reduction in the area covered by CD31+ cells. (G) Cell proliferation almost halved in the presence of a VEGF scavenging antibody (EdU incorporation for a 6 hour time window after 4 days).

\section{Cell proliferation participates in patterning vascular structures}

Tissue contraction was demonstrated to form a gradient of VEGF production which led to the preferential formation of vascular structures in regions of high deformation. To assess whether such patterns are driven by a local increase in proliferation of endothelial cells an EdU (5-ethynyl-2'- 
deoxyuridine) assay was employed. Administering EdU during a time window of 12 hours at day 4 of cell pellet co-culture marked the cells that proliferated, which mostly coincided with the endothelial marker CD31 and were localized in the tissue periphery (Figure 7E). This indicates that increased local formation of vascular structures was mostly driven by cellular proliferation. Moreover, it was observed that endogenous VEGF scavenging by administering a VEGF-A antibody during 4 days of culture led to a significant decrease in the yield of CD31+ area (Figure 7E, $p<0.05$ ) and to a reduction in number of proliferating cells (Figure 7F, EdU incorporation, $p<0.05$ ). A scratch assay did not show a dependency of cell migration on the global presence of VEGF-A (Figure S14).

\section{Discussion}

The microfabricated platform allows for the fine-tuning of the physical and biological properties of the building blocks using HTS. This permits the rapid discovery of novel conditions that direct the differentiation of spheroidal building blocks, which can be monitored using $\mathrm{HCl}$. This screening platform differentiates itself from existing methods ${ }^{15,48-52}$ by combining features, such as the high spatial resolution of microwells, high clarity for microscopy, high control over spheroidal building block formation, high replicate number of individual spheroidal building blocks per well of the culture plate, flexibility in refreshing medium and upscaling potential. In conjunction with fluorescent reporters and live staining methods, this system allows for automated read-out of phenotypical dynamics such as morphogenetic events and gene expression patterns spatially resolved on a cellular level. As such, adequate building blocks can then be used for the directed assembly of tissues in manifold. We envision applicability of this line of fabrication as a replicable method for the production of complex organotypic tissues up to the industrial scale.

The formation of stapes-like tissues demonstrates the dimensional flexibility of the bottom-up approach in building larger tissues with precisely defined shape. To even further improve the geometrical definition of, for example, a biofabricated stapes, a combination of (micro-)CT (X-ray computer tomography) with stereo lithography techniques could be used to build a more accurate 3D model. We propose that this tissue represents an important step toward the formation of fully matured and functional bone tissue via endochondral ossification. These tissues of multi-millimeter or centimeter-scale can be easily manipulated using surgical instruments and could potentially serve in clinical applications, for example by implantation to replace defective tissues or fill a critical defect. As the tissue implant is based on autologous cellular material only, there is increased freedom to integrate into the host tissue. Moreover, potentially unfavorable degradation products from scaffold materials, that might lead to side-effects such as inflammation and fibrous tissue formation, are also avoided. Gas and nutrient exchange can become an issue in such large, dense tissues. Such problems can be circumvented by precise optimization of the building blocks (e.g. metabolic activity), by designing perfusion channels into the final tissue or via the use of bioreactors. Besides clinical applications, tissues of millimeter-scale or larger may provide a means for tissuescale processes that are difficult to model in vitro, such as tissue fusion for modelling in vivo wound healing.

It is favorable to match the physical properties (stiffness and structure) of tissue constructs to their in vivo counterparts to allow, for example, bone and cartilage tissue replacement. Furthermore, to increase tissue complexity, molded tissues can function as molds themselves. For 
example, geometric tissues including internal cavities or channels might be fabricated and filled with single cells or spheroidal building blocks. Molded apertures, originating from corresponding pillars in the agarose mold, can also be used to alleviate nutrient deficiencies in tissues of larger size by providing a porous network that enhances diffusion and perfusion, as was studied previously ${ }^{53}$. However, in our approach, and for the aforementioned reasons, we intend to avoid the use of synthetic scaffold materials. To promote neovascularization, endothelial cells can be seeded that line up within these channels. In addition, the resulting tissue assemblies might be cultured on top of a vascular bed ${ }^{54}$.

Understanding how the angiogenic microenvironment orchestrates the formation of angiogenic patterns has substantial clinical implications ${ }^{33,55}$. It is recognized that a careful balance of VEGF signals, in part dependent on the ECM composition, elicits proliferative and migratory responses in endothelial cells. We demonstrated that cell-only micro-scaled building blocks can be used in a bottom-up approach to form 3D tissues that can be directed in development to recapitulate features of morphogenesis including angiogenesis. Tissue contractility locally regulated VEGF-A expression which, in turn, led to the formation of angiogenic structures. These results strongly indicate that tissue deformation contributes to the physiological patterning of angiogenesis. This suggests a role for physical forces in the long-range patterning of the vascular system.

The local cellular microenvironment influences the intensity and transmission of physical forces for which the ECM and the actin cytoskeleton are the major contributors ${ }^{56}$. When tissues are initially assembled, cellular F-actin is likely the main determinant of the mechanical properties of the tissue. In our engineered tissues, regions of high intensity F-actin were preferentially found in the periphery, similar to the presence of CD31+ structures. However, in contrast to CD31+ structures, Factin intensity within the periphery showed no difference between tissue corners and sides. As such, F-actin could not be directly related to angiogenic patterning in these tissues.

Considering the finding that ECM-bound VEGF isoforms were preferentially expressed in deforming tissues, we speculate that local differential production of ECM in our engineered tissues plays an additional factor in angiogenic patterning.

Endothelial proliferation and the associated morphogenetic aspects of angiogenesis are principally driven by VEGF signaling ${ }^{57}$. Binding of VEGF with the VEGFR2 activates downstream pathways such as the PI3K-AKT pathway, that is a strong regulator of glucose metabolism and protein synthesis ${ }^{58}$. In developing tissues a balanced regulation of VEGF function is essential since moderate overexpression is lethal ${ }^{59}$ and only short-term systemic blocking can lead to vessel regression ${ }^{60}$. The results we found suggest that local tissue compaction can form gradients of angiogenic microenvironments (VEGF signaling) and angiogenesis. Such dynamic systems of control may resemble the formation of biochemical gradients during native development that function as a driving force in tissue development and patterning. During native development, gradients are formed based on the geometry of the tissue, the composition of the tissue (e.g. presence of ECM), the cellular density, the metabolic activity of the cells or the distance to the nearest blood vessel. As such, we propose the presence of gradients as one of the strengths of our system. The formation of tissues with precise geometries is, besides its obvious mechanical functions, an additional means of 
creating and modulating physiological levels of biochemical gradients to direct tissue development and patterning in engineered constructs.

Endothelial cells typically proliferate and form new blood vessels in environments where nutrients and oxygen are low. Herein, hypoxia is a strong driver of angiogenesis through regulating the production of angiogenic factors ${ }^{61}$, primarily via hypoxia inducible factor (HIF) $1 \alpha$. Without perfusion, however, thick in vitro generated tissues often develop a necrotic core due to low exchange of gas and nutrients. Therefore, tissues were designed with dimensions limiting the lack of oxygen diffusion (tissue thickness around 250 micrometers). In case of hypoxia, the most hypoxic region and therefore the most VEGF abundant region, would presumably be found at the center of tissues. However, in contracting tissues an inverse VEGF gradient was found, which renders hypoxia as a driver of angiogenesis unlikely. This suggests that other mechanisms apart from HIF signaling regulate angiogenesis, possibly mechanotransduction.

\section{Conclusion}

In conclusion, we demonstrated an accessible and versatile microfabrication platform to build scaffold-free 3D tissues with complex architectures. The novelty of our approach lies in harnessing the power of development, using cells as the only material to build tissues via sequential assembly of multicellular building blocks. This gives tissues the freedom to self-deform, remodel, and become physiologically and functionally more akin to their native analogues. 


\section{Experimental Section}

\section{Cell culture and reagents}

Human bone marrow aspirates were obtained from donors after written informed consent, and hMSCs were isolated and expanded as previously described ${ }^{62}$. During spheroid formation and culture, human mesenchymal stromal cells and bovine primary chondrocytes (bPCs) were cultured in differentiation medium, termed McKay medium, composed of Dulbecco's Modified Eagle's Medium from Life Technologies (LT), supplemented with $10^{-7} \mathrm{M}$ dexamethasone (Sigma), $50 \mathrm{mg} \mathrm{ml}^{-1}$ ascorbate 2-phosphate, $40 \mathrm{mg} \mathrm{ml}^{-1}$ proline, $100 \mathrm{mg} \mathrm{ml}^{-1}$ pyruvate, and $50 \mathrm{mg} \mathrm{ml}^{-1} \mathrm{ITS}^{+}$Premix (Becton, Dickinson and Company, MA: $6.25 \mathrm{mg} \mathrm{ml}^{-1}$ insulin, $6.25 \mathrm{mg} \mathrm{ml}^{-1}$ transferrin, $6.25 \mathrm{ng} \mathrm{ml}^{-1}$ selenious acid, $1.25 \mathrm{mg} \mathrm{ml}^{-1}$ bovine serum albumin, $5.35 \mathrm{mg} \mathrm{ml}^{-1}$ linoleic acid), $100 \mathrm{U} \mathrm{ml}^{-1}$ penicillin (LT), and $100 \mathrm{mg} \mathrm{ml}^{-1}$ streptomycin (LT). Differentiation of hMSC derived spheroids and tissues to the adipogenic lineage was done using medium composed of DMEM (Gibco), 10\% FBS (Biowhittaker), $100 \mathrm{U} \mathrm{ml}^{-1}$ penicillin and $100 \mathrm{\mu g} \mathrm{ml}^{-1}$ streptomycin (Gibco), $0.2 \mathrm{mM}$ indomethacin (Sigma), $0.5 \mathrm{mM} \mathrm{3-}$ isobutyl-1-methylxanthine (Sigma), $10^{-6} \mathrm{M}$ dexamethasone (Sigma) and $10 \mathrm{mg} \mathrm{ml}^{-1}$ insulin (human, Sigma). Differentiation of hMSCs derived spheroids and tissues to the osteogenic lineage was done using basic hMSC medium supplemented with $10^{-8} \mathrm{M}$ dexamethasone (Sigma). Human umbilical vein endothelial cells were purchased from Lonza (Lonza Group Ltd, Switzerland) and cultured in endothelial growth medium-2 (Cambrex). The $\mathrm{C} 2 \mathrm{C} 12$ mouse myoblast, mouse embryonic fibroblast (mEF) and Chinese hamster ovary (cHO) cells were cultured in Dulbecco's Modified Eagle's Medium supplemented with $10 \%$ fetal bovine serum (FBS; LT), $100 \mathrm{U} \mathrm{ml}^{-1}$ penicillin (LT) and $100 \mathrm{mg} \mathrm{ml}^{-1}$ streptomycin (LT). Mouse embryonic stem cells were expanded on mEF cells in ES medium consisting of: Dulbecco's Modified Eagle's Medium High Glucose (LT) supplemented with $10 \%$ fetal bovine serum (FBS, Greiner), $4 \mathrm{mM}$ Glutamax (LT), $100 \mathrm{U} \mathrm{ml}^{-1}$ penicillin (LT), $100 \mathrm{mg} \mathrm{ml}^{-1}$ streptomycin (LT), $10 \mathrm{mM}$ non-essential amino acids (LT), freshly supplemented with $0.05 \mathrm{mM}$ 2-mercaptoethanol (LT), $1000 \mathrm{U} \mathrm{ml}^{-1}$ leukemia inhibitory factor (LT), $3 \mu \mathrm{M} \mathrm{CHIR99021} \mathrm{(GSK3} \beta$ inhibitor, Axon Medchem) and $1 \mu \mathrm{M}$ PD0325901 (MEK/ERK inhibitor, Sigma Aldrich).

\section{Tissue culture}

Building blocks were flushed out of the microwells after appropriate culture time, collected by centrifugation, resuspended in fresh McKay medium and seeded onto the geometric array at a density of 300 to 500 building blocks per tissue with a PA of $1 \mathrm{~mm}^{2}$. Medium was refreshed every 2 days. Tissues with primitive vascular structures were formed by culturing spheroids, consisting of a mixed population of $92 \%$ hMSCs and $8 \%$ hUVECs, for 5 days on the microwell array and 150 hours on the compensated triangle-shaped geometric array. bPCs were cultured for 2 days on the microwell array and then cultured on the geometric arrays (triangle, square, circle) for 21 days in differentiation medium (McKay) supplemented with transforming growth factor $\beta 3$ (TGF $\beta 3,10 \mathrm{ng} \mathrm{ml}^{-}$ $\left.{ }^{1}\right)$. Stapes-shaped tissues were formed using human primary chondrocytes expanded in hypoxic conditions $\left(2.5 \% \mathrm{O}_{2}\right)$. Spheroids were formed on the microwell array (140 cells/ spheroid, 2800 spheroids per microwell array chip) and cultured for 48 hours. Then, spheroids from 8 chips ( 22,000 spheroids) were seeded onto the stapes geometric array, yielding 5 stapes tissues which 
were fixated using $4 \% \mathrm{w} / \mathrm{v}$ paraformaldehyde after 72 hours. Spheroids and stapes tissues were cultured in McKay medium including $10 \mathrm{ng} \mathrm{ml}^{-1} \mathrm{TGFb} 3$ and refreshed every 2 days.

\section{Kinase inhibitor screening}

The kinase inhibitor library V2.2 ( 80 chemicals reconstituted at $10 \mathrm{mM}$ in DMSO) from Enzo Life Sciences was used to screen for compounds that modulate H2B-GFP reporter activity in EBs from a mouse embryonic stem cell line comprising a PDGFRa ${ }^{\mathrm{H} 2 \mathrm{~B}-\mathrm{gfp} /+}$ knock-in allele (a histone H2B-GFP fusion protein reporter under the control of PDGFR $\alpha$ regulatory elements ${ }^{29}$ ). In each well, we aimed to seed 5590 cells, which equates to an average of 13 cells per microwell, and allowed to aggregate

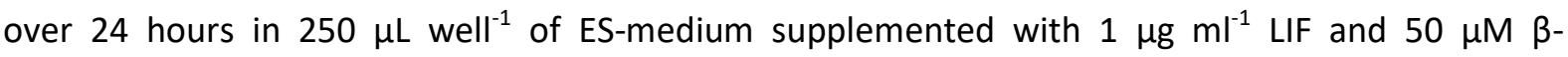
mercaptoethanol. After 24 hours, $200 \mu \mathrm{L}$ of medium was refreshed with ES-medium supplemented with $50 \mu \mathrm{M} \beta$-mercaptoethanol and the kinase inhibitor drugs were administered in a working concentration of $20 \mu \mathrm{M}$. After 72 hours, $100 \mu \mathrm{L}$ of medium was refreshed with ES-medium supplemented with $50 \mu \mathrm{M} \beta$-mercaptoethanol, and the kinase inhibitor drugs were administered again at a concentration of $20 \mu \mathrm{M}$. RA was used as a positive control for H2B-GFP expression ${ }^{27}$. After 96 hours, the EBs were fixated in 4\% paraformaldehyde, washed thoroughly in PBS, stained with $0.7 \mathrm{\mu g} \mathrm{ml}^{-1}$ DAPI solution (Sigma Aldrich, D9542), washed thoroughly in PBS and imaged using the image-montage function and a 10x objective on the BD Pathway 435. Phenotypic data were acquired from montaged images using a custom-made pipeline in CellProfiler2.0 (Broad Institute, ${ }^{63}$ ). The yield of EBs with cells positive for PDGFR $\alpha$-H2B-GFP was determined using an intensity threshold on approximately $130 \mathrm{EBs}$ per condition (i.e. per well). Compounds that were autofluorescent or images that were not in focus were omitted of analysis (13 out of 96 conditions).

\section{Immunohistochemical analysis}

After harvesting, tissues were frozen in Cryomatrix at $-60^{\circ} \mathrm{C}$. Sections $(7 \mu \mathrm{m})$ were prepared on a cryotome. Sections were fixed in cold acetone for $5 \mathrm{~min}$, air-dried, and rehydrated for $10 \mathrm{~min}$, after which they were incubated for 30 min with $10 \%$ FBS in PBS to block nonspecific background staining. Sections were incubated with monoclonal mouse anti-human CD31 antibody (Dako, 1:20) for 1 hour. Sections were washed in PBS and incubated with the secondary antibody (Alexa Fluor 488, Invitrogen). Samples were counterstained with DAPI. Pictures were acquired on an inverted confocal microscope (Leica LSM500). Cartilage formation was assessed by Safranin-O staining according to standard protocols.

\section{VEGF protein measurements using ELISA}

Comparatively large cell pellets (approximately $1.5 \mathrm{~mm}$ in diameter) were cultured for different time points (3, 6 and 9 days) before the VEGF protein content in the supernatant was measured using ELISA according manufacturer's protocol (Human VEGF Quantikine ELISA Kit, R\&D Systems).

\section{Gene expression analysis by qPCR}

The effect of tissue contraction and deformation on expression of angiogenic marker genes was analyzed from tissues cultured for 3 days on the geometrical arrays. RNA was isolated from a pool of 5 tissues by snap freezing the tissues in liquid nitrogen, crunching them using a pestle and by using 
an RNeasy mini kit (Qiagen). qPCR was performed by using SYBR green (Invitrogen) on a Light Cycler (Roche). Expression of angiogenic markers was calculated relative to DAPDH hRNA levels by the comparative $\triangle C T$ method. Primers were obtained from SAS Bioscience and used according to the manufacturer's protocol.

\begin{tabular}{lll}
\hline Gene & Forward primer & Reverse primer \\
\hline Human VEGF-121 & GCGGATCAAACCTCACCAAG & TCGGCTTGTCACATTTTTCTTG \\
Human VEGF-145 & GAATGCAGACCAAAGAAAGATAGAG & TCGGCTTGTCACATACGCTCC \\
Human VEGF-165 & ATCTTCAAGCCATCCTGTGTGC & CAAGGCCCACAGGGATTTTC \\
Human VEGF-189 & ATCTTCAAGCCATCCTGTGTGC & CACAGGGAACGCTCCAGGAC \\
\hline
\end{tabular}

Table 1: Sequences of primers for VEGF isoforms 


\section{Supplementary information}

\section{Materials and methods}

\section{Microfabrication of screening array}

A polymethylmethacrylate (PMMA) platform (1 mm high), including through-holes $(5 \mathrm{~mm}$ in diameter) in a patterned arrangement of a standard 96wp, was clamped onto an SU-8-patterned silicon wafer. A reservoir on top of the PMMA platform was used to cumulate a mixture of PDMS base resin and curing agent (10:1, Sylgard 184, Dow Corning). The whole assembly was degassed in a vacuum chamber prior to incubation in an oven at $65^{\circ} \mathrm{C}$ for 2 hours to cure the PDMS mixture. The obtained elastomeric stamp was then used to imprint microwells in a thin layer of pre-boiled liquid agarose (2.5\% w v $\mathrm{v}^{-1}$ in PBS, ultra-pure agarose, Invitrogen) inside the wells of a $96 \mathrm{wp}$ ( $70 \mu \mathrm{L}$ per well, dispensed using an electronic multi-channel pipette). The plate, with the agarose solution inside and the stamps on top, was then directly centrifuged ( $2500 \times \mathrm{g}, 30 \mathrm{~s}$ ). Of note, stamps and $96 \mathrm{wp}$ were pre-heated to $55^{\circ} \mathrm{C}$ using a hotplate to prevent spontaneous solidification of the agarose solution already during liquid dispensing. One stamp covered three columns and eight rows of a 96wp, thus, four stamps covered a full 96wp. After solidification of the agarose hydrogel, the assembly was submerged into Milli-Q (Millipore) water to aid the release of the stamps. The screening array plates were sterilized in 70\% ethanol for 15 min and washed thrice in PBS inside a laminar flow cabinet. After wetting and incubation in the corresponding cell culture medium ( $200 \mu \mathrm{L}, 1$ hour), the medium was substituted by a concentrated suspension of cells (200 to $250 \mu \mathrm{L}$ ).

\section{Microfabrication of microwell array and geometric array}

Micropatterned agarose chips for non-adherent cell culture were formed by replica molding. Patterned elastomeric stamps of PDMS were replicated from either etched silicon wafers or SU$8 /$ silicon wafers according to established protocols ${ }^{64}$ and sterilized for $10 \mathrm{~min}$ in $70 \%$ ethanol. A 3\% agarose solution (ultra-pure agarose, Invitrogen) was cast onto the PDMS stamp, de-molded upon solidification, cut to size and placed in a $12 \mathrm{wp}$ (Figure 1). After wetting in the corresponding differentiation medium (500 $\mu \mathrm{L}$ ), a concentrated suspension of cells was seeded and allowed to settle for $15 \mathrm{~min}$. Excess cells were rinsed away with culture medium and $1.5 \mathrm{~mL}$ of medium was added. Half of the medium was exchanged every day of culture. Upon culture on the microwell array, spheroids were gently flushed out using culture medium, centrifuged ( $300 \times \mathrm{g}, 1 \mathrm{~min}$ ), resuspended in the culture medium and seeded on the geometric arrays. Spheroids were allowed to settle in the wells for $1 \mathrm{~min}$ and any excess was harvested by washing and dispensed again for optimal seeding. The seeded chips were centrifuged ( $300 \times \mathrm{g}, 1 \mathrm{~min}$ ) and $1.5 \mathrm{~mL}$ of culture medium was added. Half of the medium was refreshed every day of culture.

\section{Measuring tissue properties}

Cellular density was measured using a Radial Profile Plot. Image J plugin by Paul Baggethun. This plugin produces a profile plot of normalized integrated intensities around concentric circles as a function of distance from a point in the image. The intensity at any given distance from the point represents the sum of the pixel values around a circle. This circle has the point as its centre and the 
distance from the point as radius. The integrated intensity is divided by the number of pixels in the circle that is also part of the image, yielding normalized comparable values. The profile $x$-axis can be plotted as pixel values or as values according to the spatial calibration of the input image. See http://rsb.info.nih.gov/ii/plugins/radial-profile.html.

\section{Relative diameter of building blocks}

The relative diameter of building blocks was calculated as

$$
D=2 * \sqrt{\frac{[\mathrm{PA}]}{\pi}}
$$

\section{Circularity of tissues}

The circularity of tissues was measured using Image J. A picture was taken and a mask of each tissue was made as described for the measurement of the projection area. The circularity of the mask was measured as

$$
\text { Circularity }=\frac{4 * \pi *[P A]}{[\text { Perimeter }]^{2}}
$$

\section{Maintenance of tissue shape}

Maintenance of tissue shape was graded by quantifying the overlapping area (OA) of the projected area of the tissue with a corresponding perfect shape (e.g. square, circle) of identical surface area. Shape factor $=$ OA.

\section{Morphological measurements of EBs}

Morphological measurements of EBs were done using CellProfiler 2.0 ${ }^{[27]}$. Each well of the screening array was imaged on-chip by stitching 25 pictures $(5 \times 5,10 x$ objective) using a BD Pathway 435 automated microscope. Background subtraction of images was done using a custom script in Matlab 2012.

\section{Projection area of tissues}

Pictures of the tissues were taken using a Nikon Eclipse TE300 microscope equipped with Nikon Digital Sight DS Fi1 digital camera and imaging system. Images were imported into Image J and a black-and-white mask picture was created using a threshold. The resulting picture was analyzed using Image J to measure the projection area (PA) of each tissue.

\section{Fluorescent reporter intensity}

ESCs with a knock-in allele GFP reporter for Sox17 and a TdTomato reporter for Nanog were allowed to aggregate into EBs for 24 hours, cultured until the appropriate time point $(0,48,72,96$ hours), washed thrice in PBS, live-stained using Hoechst 33342 (diluting stock of $10 \mathrm{mg} \mathrm{ml}^{-1}$ by $300^{-1}$, incubating for $30 \mathrm{~min}$ at $37{ }^{\circ} \mathrm{C}$, Life Technologies) or CellMask Deep Red Plasma membrane stain $\left(4000^{-1}\right.$ dilution for $10 \mathrm{~min}$ at $37^{\circ} \mathrm{C}$, Life Technologies, C10046) washed thrice in PBS and imaged 
using the BD Pathway 435 (10x objective). Exposure times, lamp intensity and dynamic range settings were kept constant. For calculating the fluorescence intensity, the average intensity was taken from the median value of 3 replicates, each consisting of about 15 EBs. In Figure 4A, a straight line was fitted on the data points, except for Nanog RA onto which a second polynomial curve was fitted.

\section{Immunocytofluorescence and analysis}

Tissues were frozen on glass slides in Cryomatrix at $-80{ }^{\circ} \mathrm{C}$. Sections $(7 \mu \mathrm{m})$ were cut using a cryotome machine. Sections were fixated in cold acetone for $5 \mathrm{~min}$ and air-dried. Sections were rehydrated for $10 \mathrm{~min}$, after which they were incubated for 30 min with $10 \%$ FBS or with host species-specific serum of the secondary antibody in PBS to block nonspecific background staining. Sections are incubated with monoclonal mouse antihuman CD31 antibody (Dako), a monoclonal rabbit antihuman VEGF Carboxyterminal end antibody (Abcam), Phalloidin-Alexa fluor 488 conjugated antibody. Sections were washed in PBS and subsequently incubated with the secondary antibody (Alexa Fluor 488 or 594 antibody, Invitrogen). Samples were counterstained with Dapi (Sigma). Cellular proliferation was performed using the Click-iT ${ }^{\mathrm{TM}}$ EdU kit (Invitrogen).

To prevent biased measurements, quantification of the area of CD31+ cells in Figure 6D was done using a threshold applied on images based on size and circularity (Image J) to select for the CD31+ structures which elongated in the main plane of the tissue. Thresholded CD31+ structures were termed vascular structures (VS). For quantification of Figure 7B, each image was divided into 20 boxes from the center to the periphery. Each box was used for quantification of the number of nuclei and the area of VS. Figure 6D Shows values in five consecutive regions ranging from the tissue center to the tissue corners, of VS density (scale from 1 to 4.8) and VS/ nucleus density (scale from 1 to 2.5 ) normalized to the center region.

\section{Results}

Circle

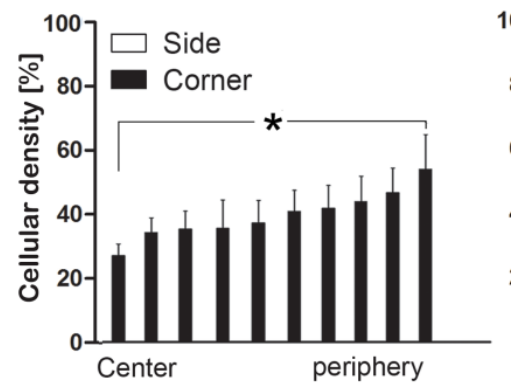

Square

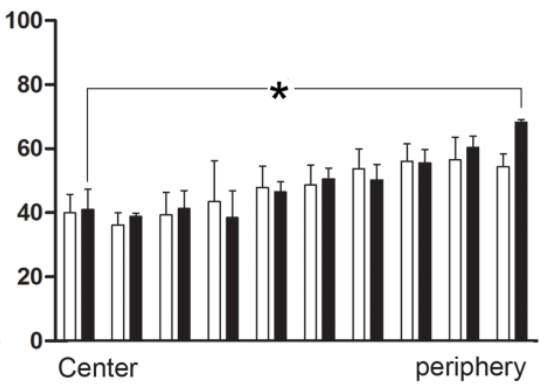

Triangle

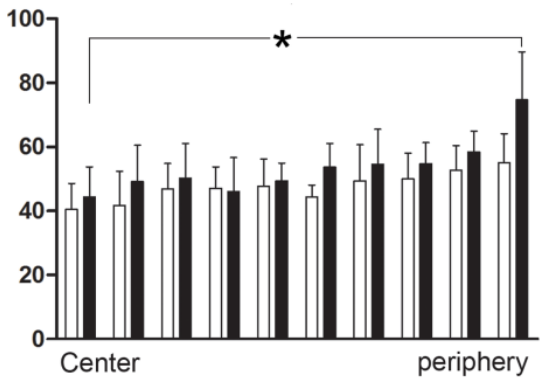

Figure S1: Heterogeneity in cellular density. Upon contraction and remodeling, the cellular density was higher in the periphery and in the corners as compared to the center (* denotes $p<0.05$ ). Tissues were built from building blocks with a mixed population of $94 \%$ hMSCs and $6 \%$ hUVECs. Building blocks were cultured for 5 days on the microwell array and 150 hours on geometric arrays. 

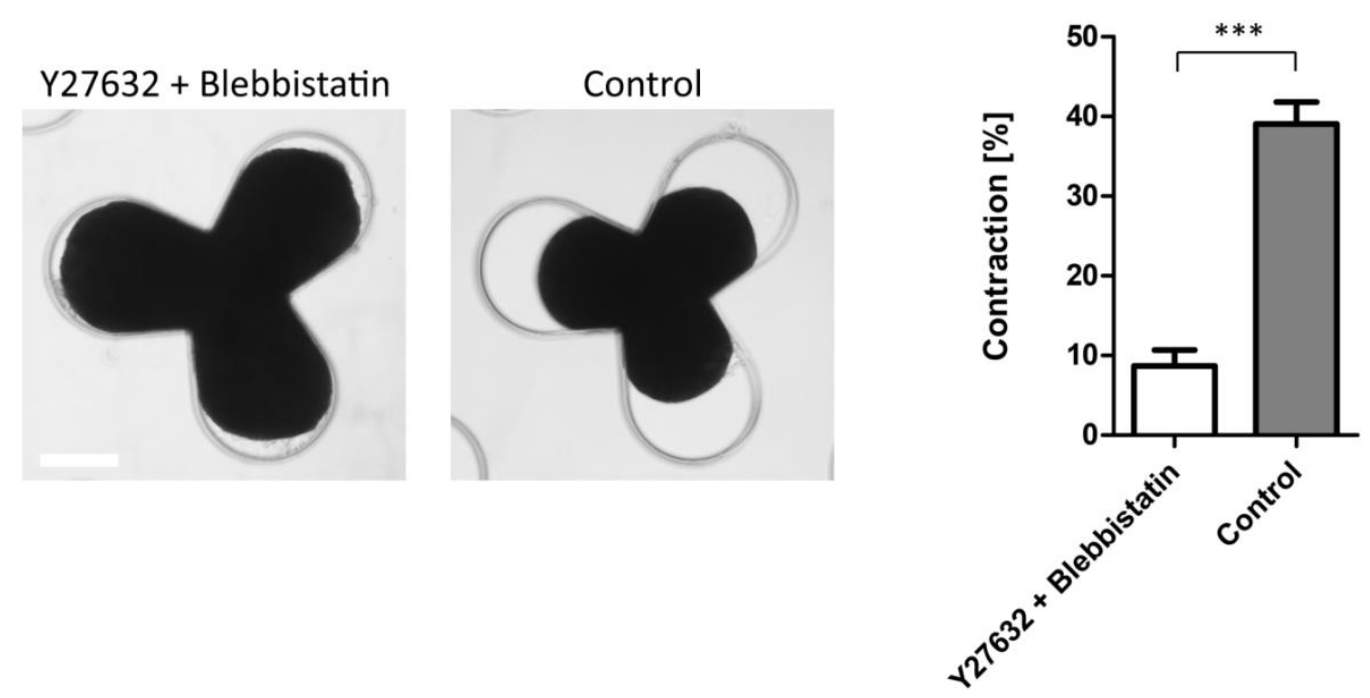

Figure S2: Trifolium-shaped tissues treated with inhibitors for actin and myosin (10 $\mu \mathrm{M}$ Y-27632 (Calbiochem) and $50 \mu \mathrm{M}$ blebbistatin (Sigma-Aldrich) added at 24 hours on the geometric array) showed impaired contraction and shape shifting $(p<0.001, n=6)$. Scale bar represents $250 \mu \mathrm{m}$.

a

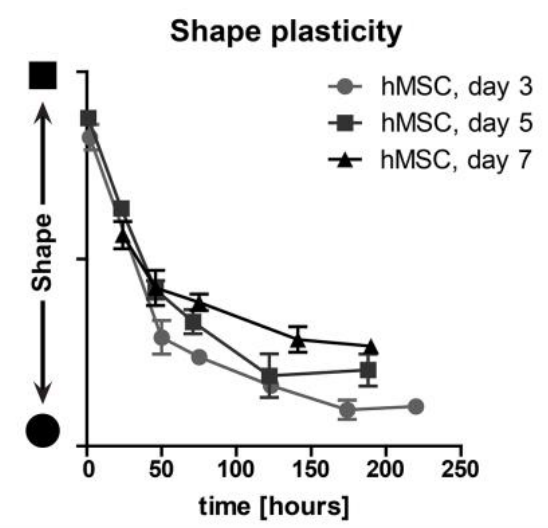

b

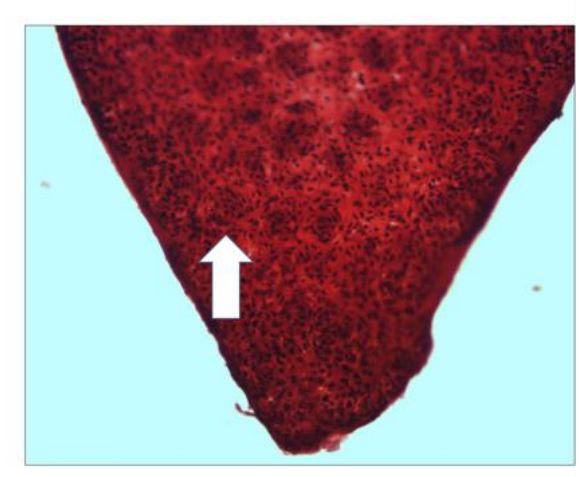

Figure S3: Uniform distribution of cells.

a) Longer culture periods of hMSC spheroidal building blocks led to increased maintenance of tissue shape over time.

b) Histological image of a triangular tissue showing fusion of chondrocyte spheroids with an incomplete flow into a uniform tissue (discretization, arrow) due to spheroids that were cultured for extended period on the microwell array. 

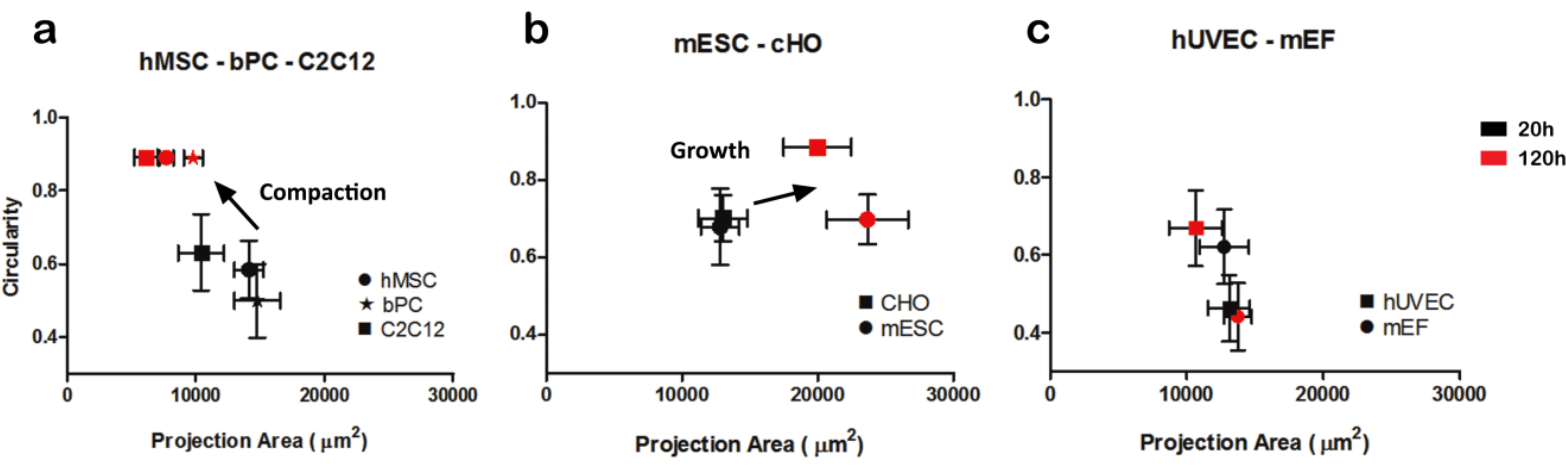

Figure S4: Building block classification by cell type

a) We found that we could classify the stereotypical behaviors into 3 categories. hMSCs, bPCs and C2C12 cells showed a decreasing projection area (PA) and increasing circularity (compaction).

b) mESCs and cHO cells showed an increasing PA (growth).

c) hUVECs and mouse embryonic fibroblasts (MEFs) showed a stable PA and circularity. 


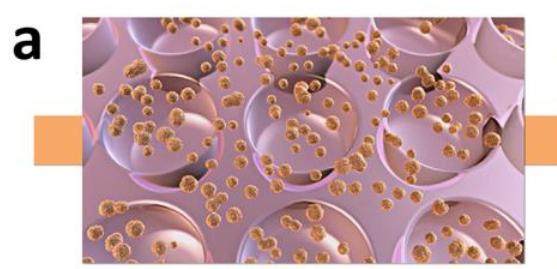

Seeding cells

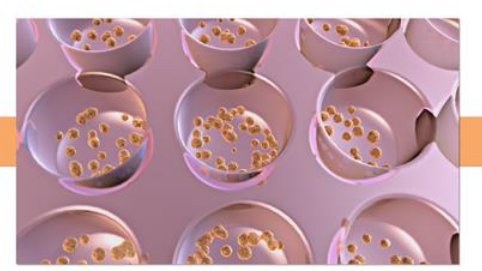

Aggregation

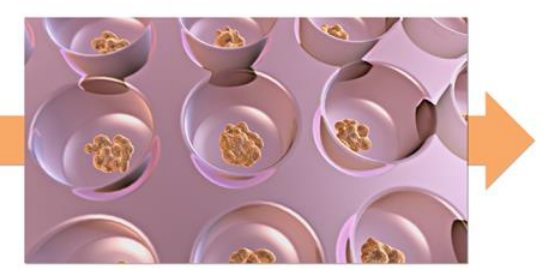

Spheroidal building blocks

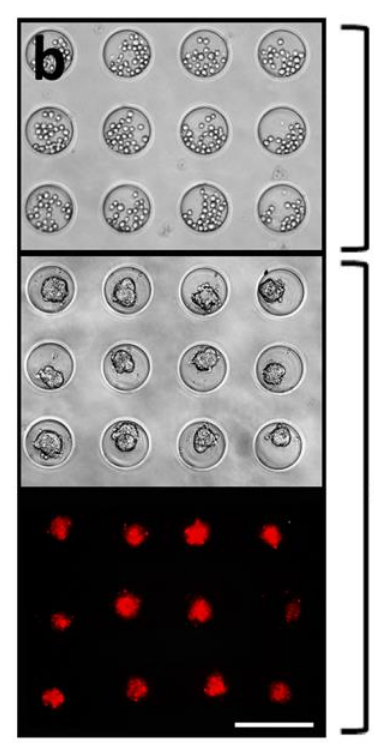

d

e

Oh
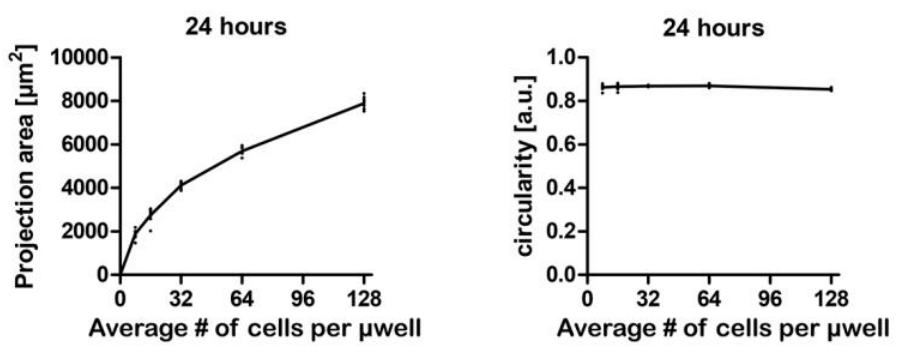

$24 h$
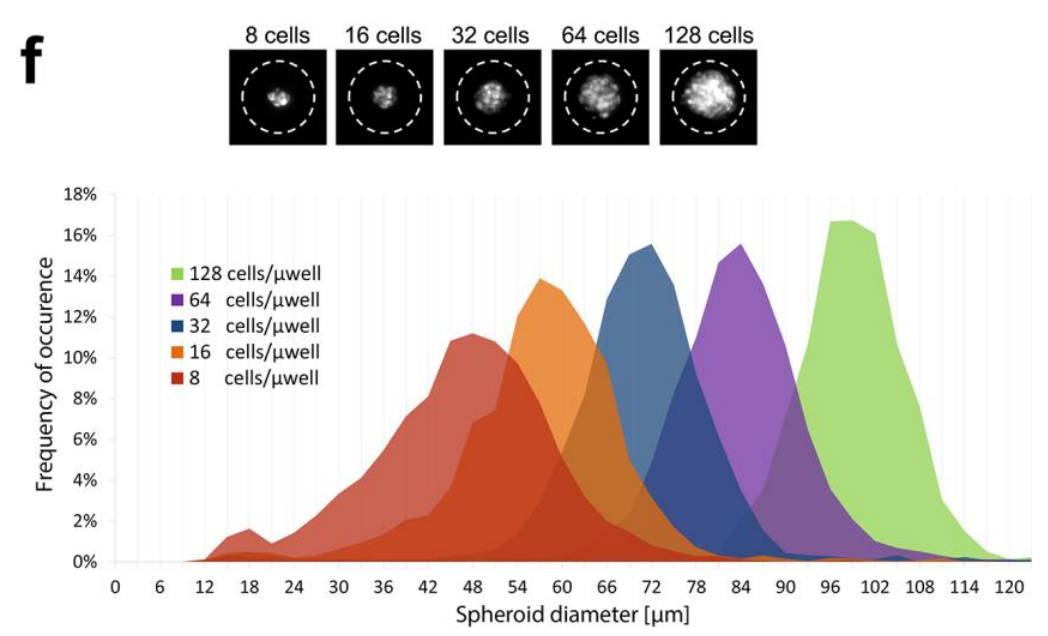

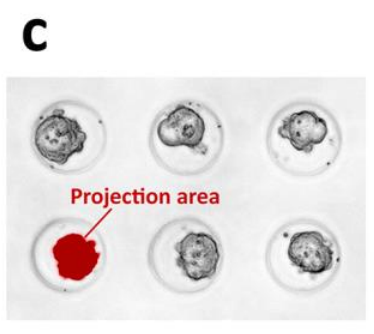

Figure S5: Building block formation and phenotypical characterization

a) Cells were seeded in bulk on the screening array, after which they spontaneously settled and aggregated into spheroidal building blocks.

b) mESCs including an H2B-RFP reporter aggregated into EBs within 24 hours. Scale bar represents 200 $\mu \mathrm{m}$.

C) Automated image analysis of phenotypic parameters on-chip. The projection area was measured as a proxy for EB-size.

d) Well-to-well variations (median values from $n=8$ wells) of the projection area related to the number of cells seeded. The maximum deviation was $6 \%$ when an average cell density of 32 to 128 cells per microwell was seeded. This deviation increased slightly when a lower density of 8 or 16 cells/microwell was seeded ( 16 and $11 \%$, respectively).

e) Well-to-well variations of the circularity (median values from $n=8$ wells) showed a reproducible circularity.

f) The distribution of the relative diameter of spheroidal building blocks was controlled by the initial number of cells seeded. The diameter of the EBs could be easily controlled by changing the cell 
Chapter 2

seeding density with an optimal, narrower distribution of diameters for a number of cells higher than 32 per microwell (frequency of occurrence of the average number of cells higher than $15 \%$ ). 


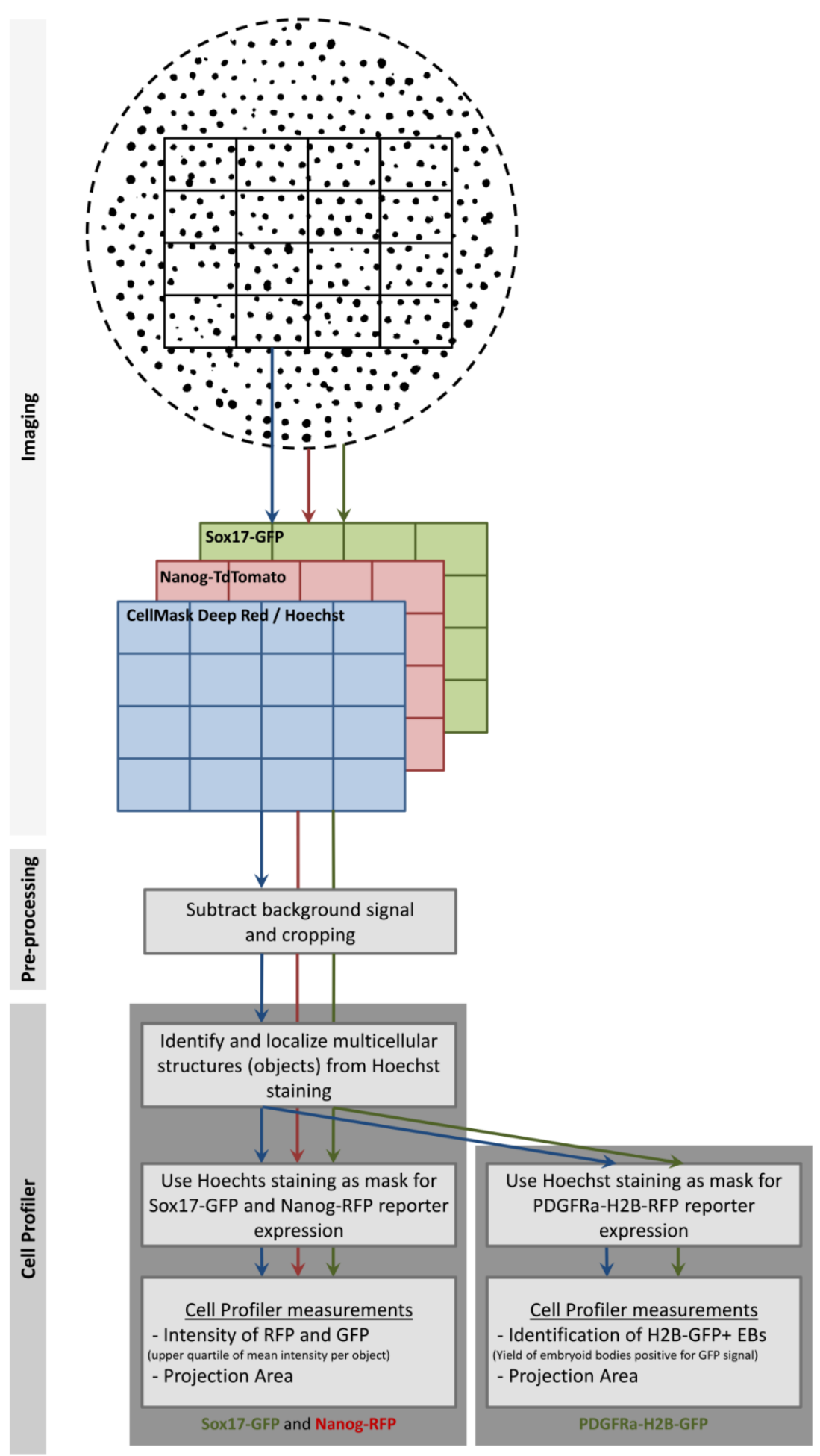

Figure S6: Process of high-content screening (HCS). Montage images for different color channels were captured using an automated fluorescence microscope (BD Pathway systems). Images were pre-processed by subtracting background and cropping. Cell Profiler 2.0 software [27] was used to identify building blocks and measure fluorescent reporter expression. 
Safranin-0

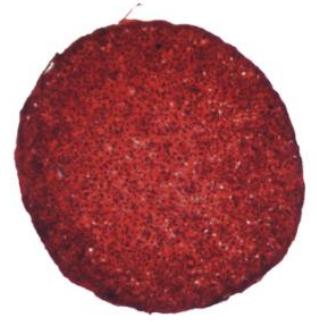

Circle

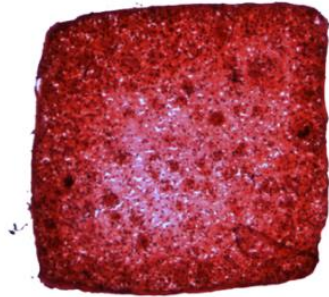

Square
21 days

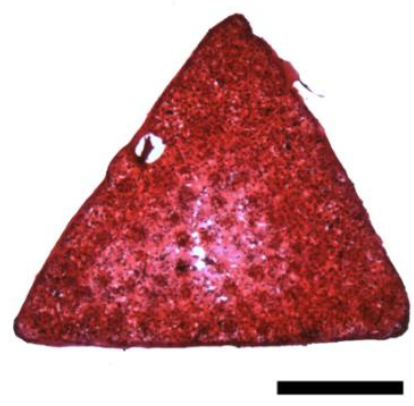

Triangle

Figure S7: Self-scaffolding of tissues. Safranin-O staining on primitive shapes showed extensive deposition of ECM. Scale bar represents $500 \mu \mathrm{m}$.

a
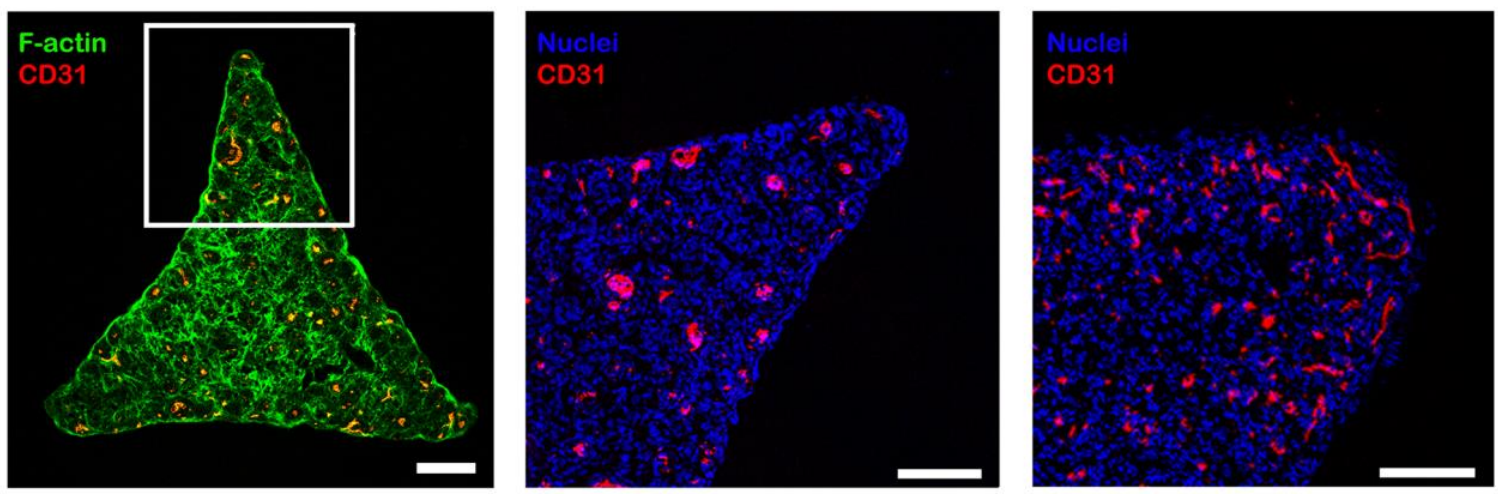

b
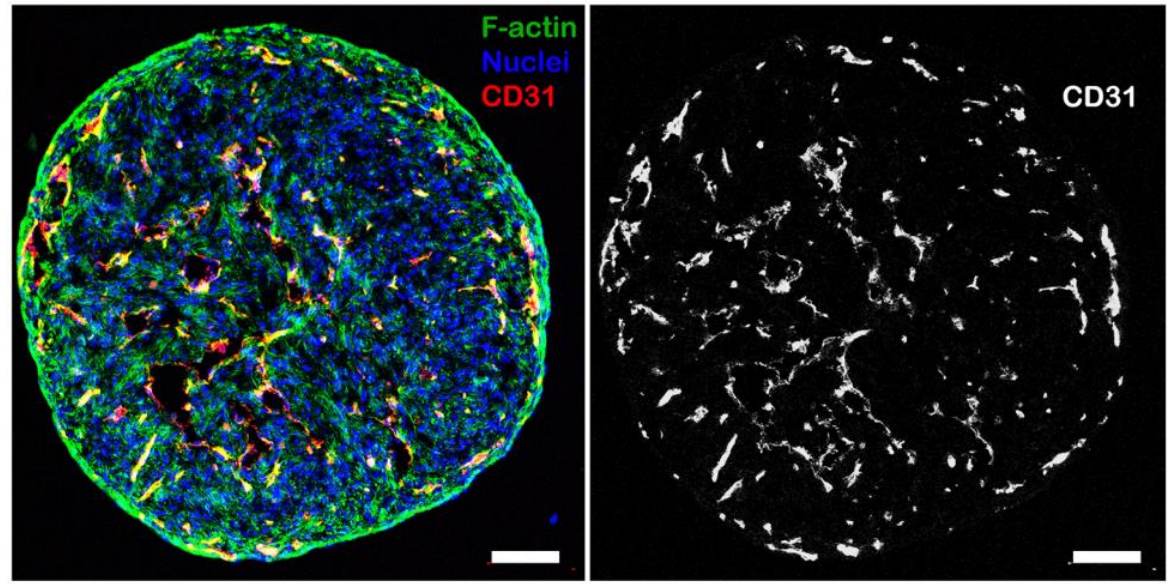

\section{Figure S8: Formation of primitive vascular structures}

a) Primitive vascular structures formed over time in tissues built from tissue building blocks consisting of a mixed population of $94 \%$ hMSCs and $6 \%$ hUVECs. Building blocks were cultured for 5 days on the microwell array and 24 to 150 hours on compensated triangle shaped geometric arrays using McKay medium. Scale bars represent $200 \mu \mathrm{m}$.

b) Vasculogenic patterns formed by adding the morphogen Sonic Hedgehog to McKay medium (spherical tissue of $400 \mu \mathrm{m}$ in diameter, cultured for 288 hours). Scale bars represent $50 \mu \mathrm{m}$. 

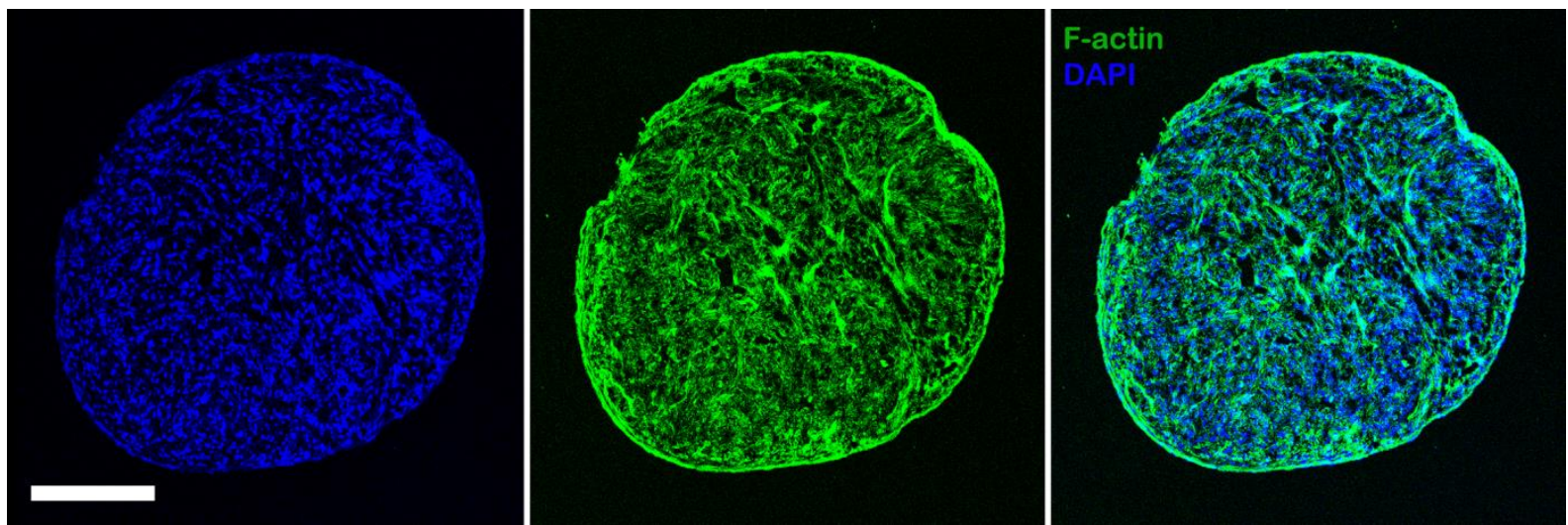

Figure 59: Uniform distribution of cells (DAPI in blue, F-actin in green) in tissues formed with mesenchymal stem cells and endothelial cells, grown in McKay medium. Scale bar represents $100 \mu \mathrm{m}$.
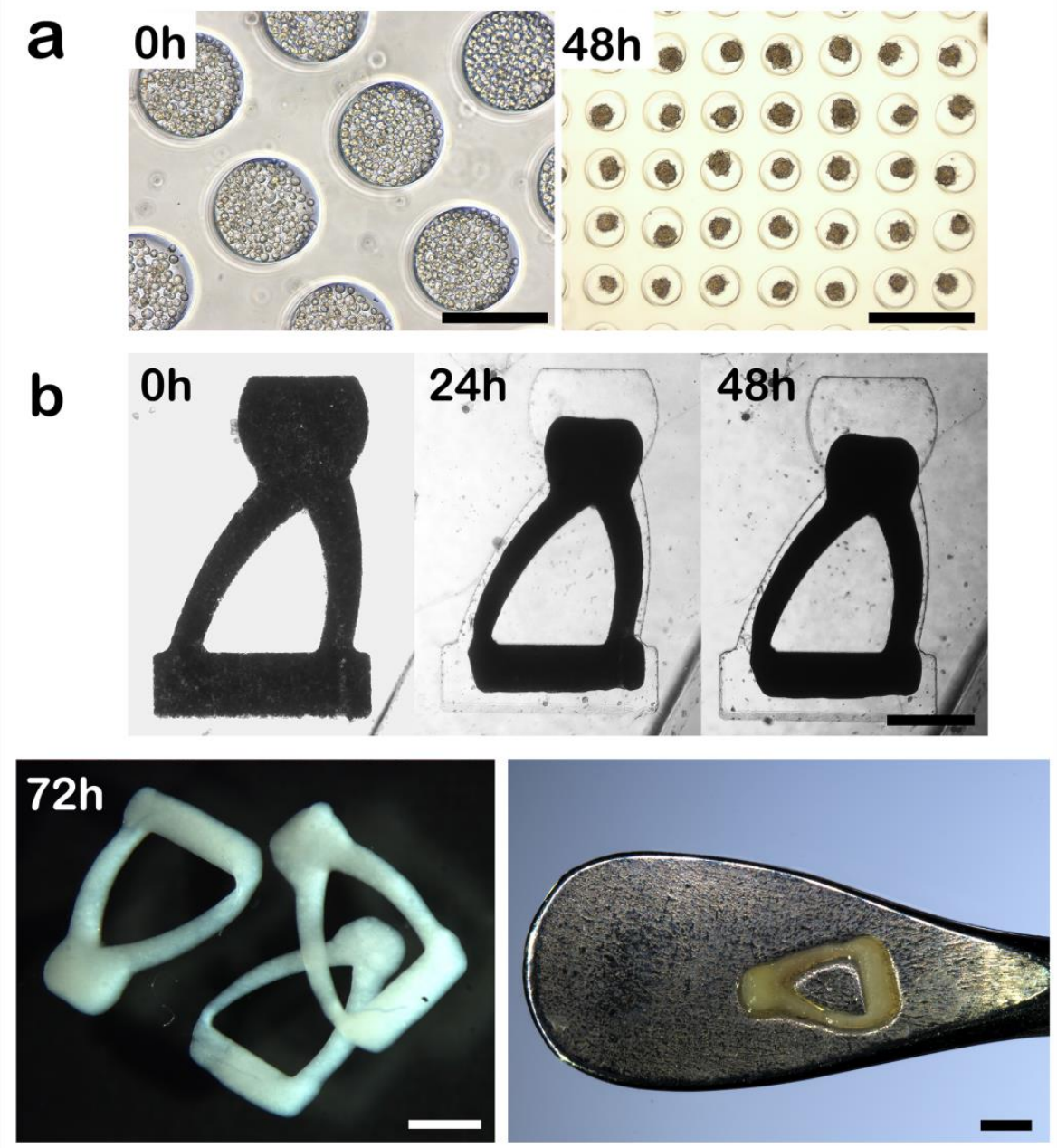

Figure S10: Formation of the stapes-shaped tissues

a) Human primary chondrocytes were seeded onto the microwell array, formed spheroidal building blocks and were cultured for 48 hours before being flushed out. Scale bars are $200 \mu \mathrm{m}$. 
b) The spheroidal building blocks were seeded onto the stapes-shaped geometric array and allowed to self-assemble and fuse over time. After 72 hours on the geometric array, stapes-shaped tissues were fixated and taken out using forceps. Scale bars are $1 \mathrm{~mm}$.

Surface morphology

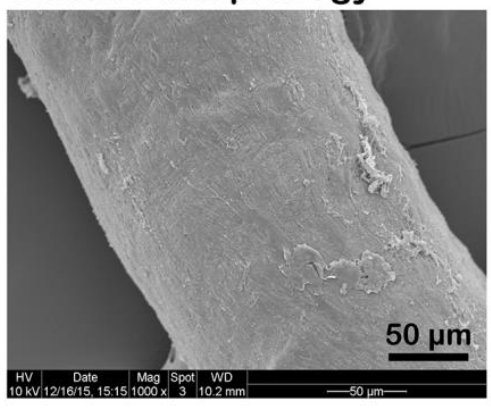

Inner morphology

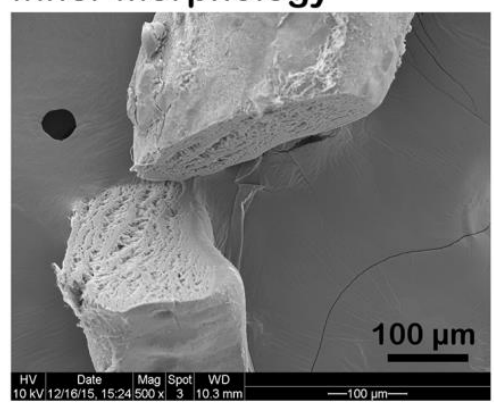

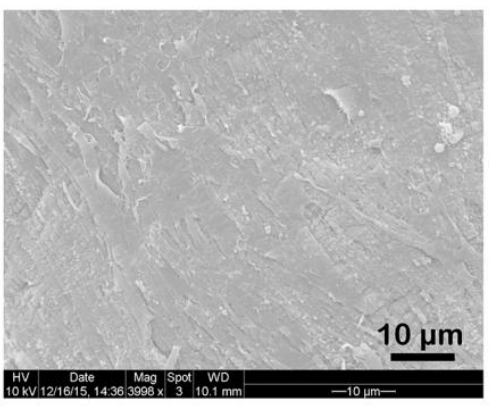
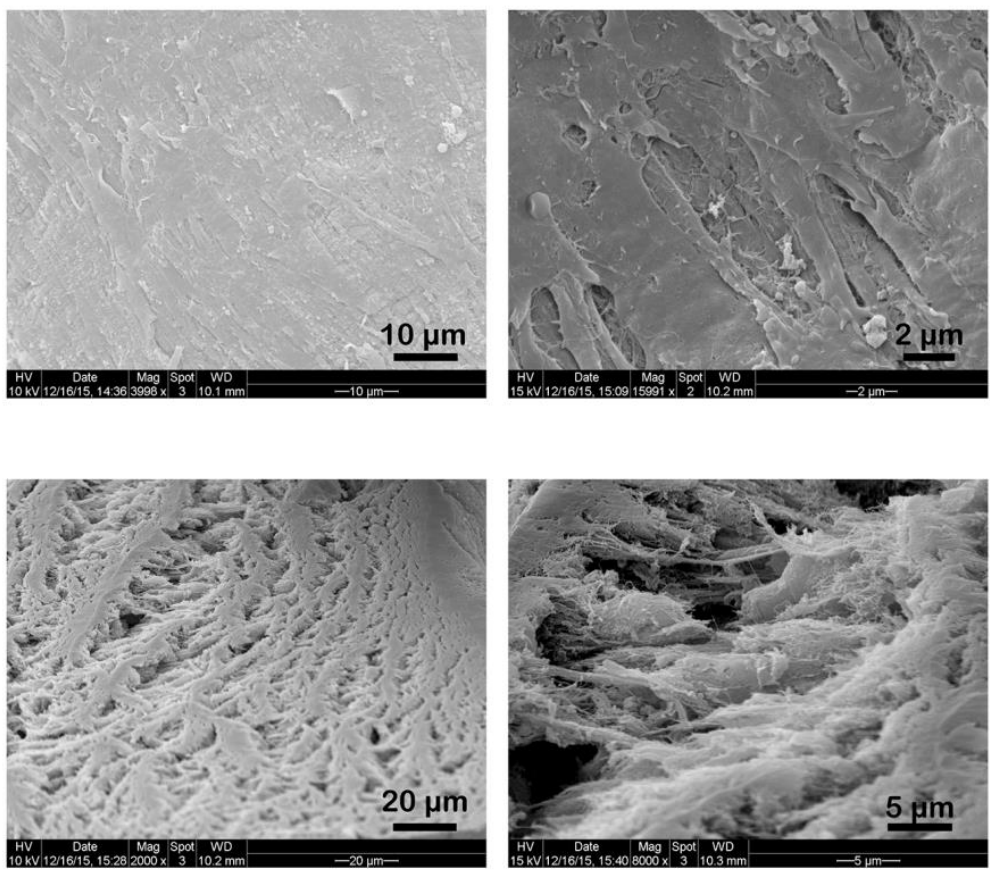

Figure S11: Stapes morphology by scanning electron microscopy showed a smooth outer surface and an inner fibrillar structure typical of the extra-cellular matrix. 
A

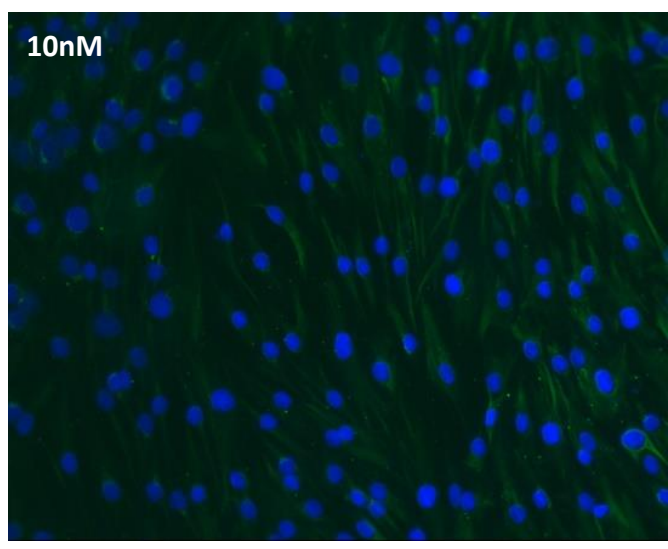

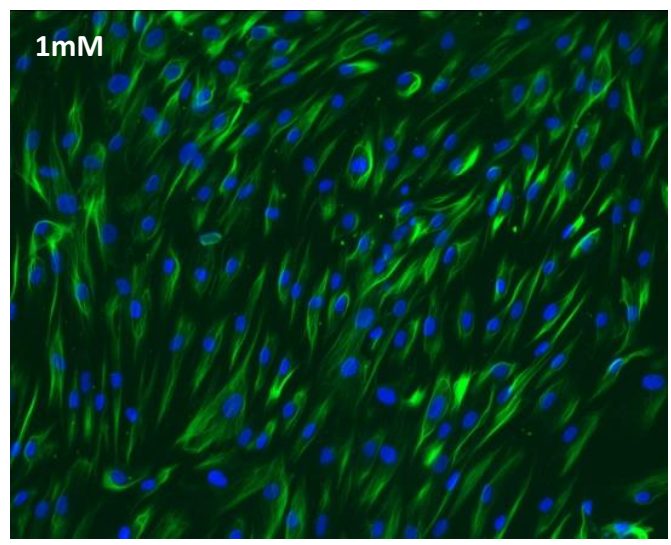
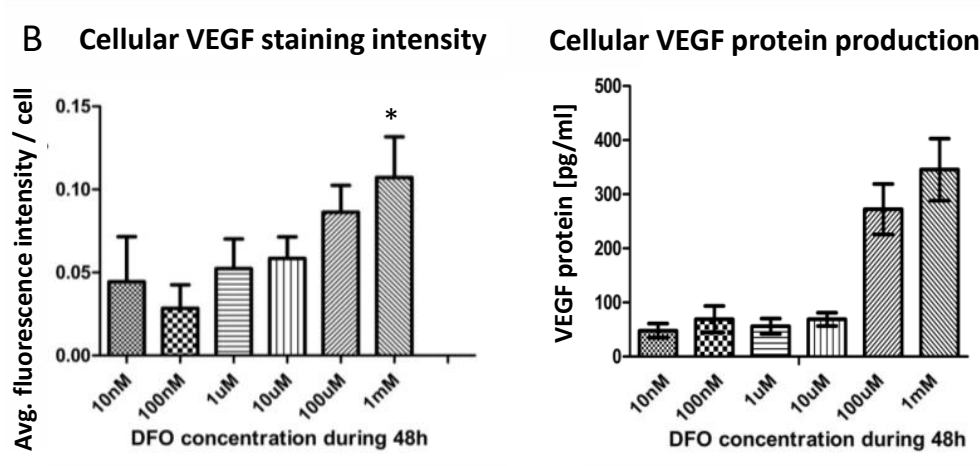

Correlation

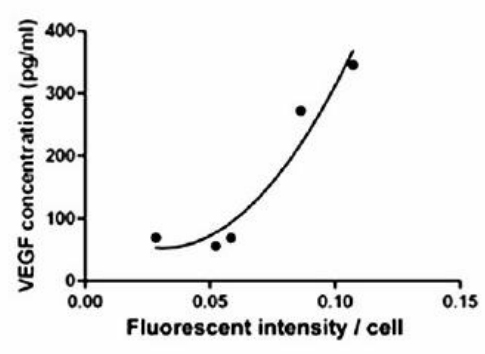

Figure S12: VEGF immunofluorescence intensity positively correlated with the concentration of DFO. (A) representative immunofluorescence images for $10 \mathrm{nM}$ and $1 \mathrm{mM}$ DFO. (B) Cellular VEGF intensity and cellular VEGF production showed a significant increase with increasing concentrations of DFO. ${ }^{*}$ denotes $p<0.05$ and *** denotes $p<0.001$ compared to $0.01 \mu \mathrm{M}$, ANOVA with Tukeys multiple comparison post-test.

\section{Normalized to lowest overall control}

\section{Expression of Vegf isoforms}

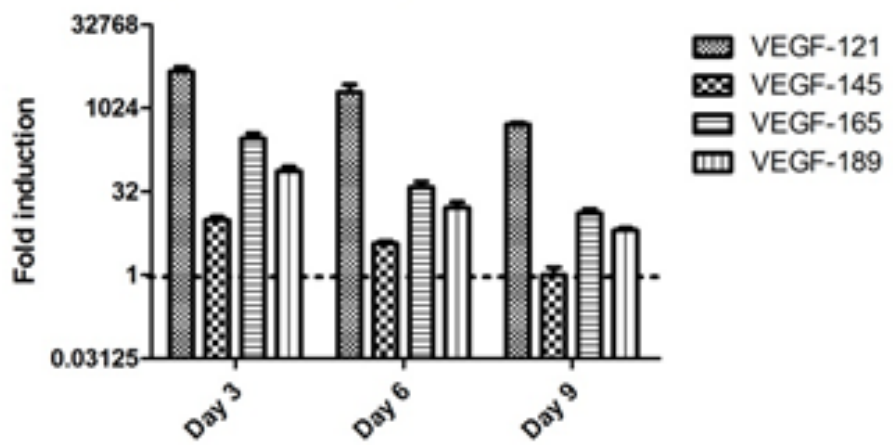

Figure S13: Mutual comparison of VEGF isoform expression in contracting tissues. Expression of mRNA product for VEGF-121, 145, 169 and 189 proteins in contracting tissue pellets normalized to the overall lowest value of mRNA expression (VEGF-145, day 9) in compacted tissues. 


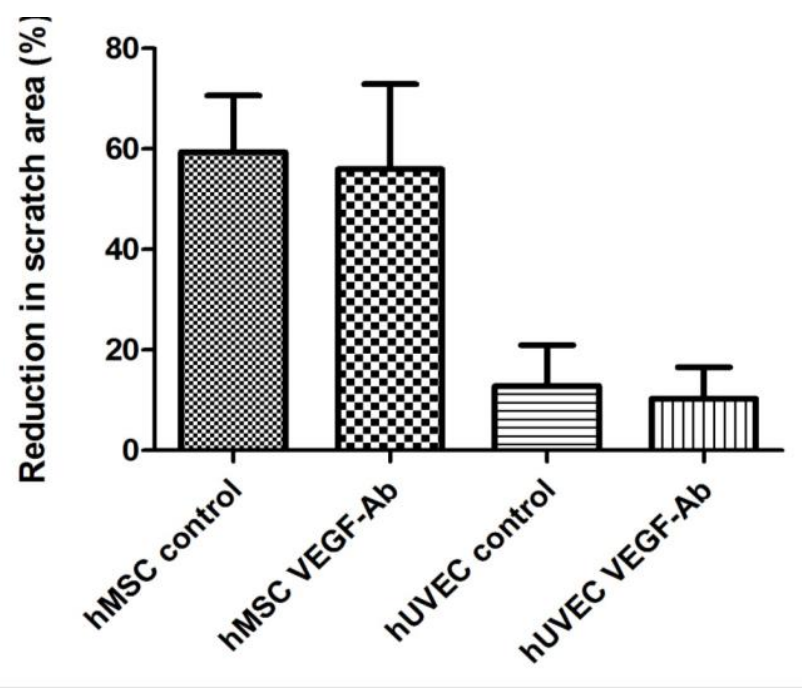

Figure S14: No signification effect on cell migration was observed from the addition of a VEGF-A-capturing antibody during culture (6 hour scratch assay).

\section{References}

1. Rivron, N.C. et al. Tissue deformation spatially modulates VEGF signaling and angiogenesis. Proceedings of the National Academy of Sciences of the United States of America 109, 6886-6891 (2012).

2. Ranga, A. et al. 3D niche microarrays for systems-level analyses of cell fate. Nature communications $\mathbf{5}$, 4324 (2014).

3. Allazetta, S., Kolb, L., Zerbib, S., Bardy, J. \& Lutolf, M.P. Cell-Instructive Microgels with Tailor-Made Physicochemical Properties. Small 11, 5647-5656 (2015).

4. Mosiewicz, K.A. et al. In situ cell manipulation through enzymatic hydrogel photopatterning. Nature materials 12, 1072-1078 (2013).

5. Nelson, C.M. et al. Emergent patterns of growth controlled by multicellular form and mechanics. Proceedings of the National Academy of Sciences of the United States of America 102, 11594-11599 (2005).

6. Kelm, J.M., Ittner, L.M., Born, W., Djonov, V. \& Fussenegger, M. Self-assembly of sensory neurons into ganglia-like microtissues. Journal of biotechnology 121, 86-101 (2006).

7. Nelson, C.M. \& Bissell, M.J. Of extracellular matrix, scaffolds, and signaling: tissue architecture regulates development, homeostasis, and cancer. Annual review of cell and developmental biology 22, 287-309 (2006).

8. Nichol, J.W. \& Khademhosseini, A. Modular Tissue Engineering: Engineering Biological Tissues from the Bottom Up. Soft matter 5, 1312-1319 (2009).

9. Liu, J.S. \& Gartner, Z.J. Directing the assembly of spatially organized multicomponent tissues from the bottom up. Trends in cell biology 22, 683-691 (2012).

10. Guven, S. et al. Multiscale assembly for tissue engineering and regenerative medicine. Trends in biotechnology (2015).

11. Pataky, K. et al. Microdrop Printing of Hydrogel Bioinks into 3D Tissue-Like Geometries. Advanced Materials 24, 391-+ (2012).

12. Kolesky, D.B. et al. 3D bioprinting of vascularized, heterogeneous cell-laden tissue constructs. Adv Mater 26, 3124-3130 (2014).

13. Pati, F. et al. Printing three-dimensional tissue analogues with decellularized extracellular matrix bioink. Nature communications 5, 3935 (2014).

14. Gurkan, U.A. et al. Engineering anisotropic biomimetic fibrocartilage microenvironment by bioprinting mesenchymal stem cells in nanoliter gel droplets. Molecular pharmaceutics 11, 2151-2159 (2014).

15. Stevens, K.R. et al. InVERT molding for scalable control of tissue microarchitecture. Nature communications 4, 1847 (2013). 
16. Leferink, A. et al. Engineered micro-objects as scaffolding elements in cellular building blocks for bottom-up tissue engineering approaches. Adv Mater 26, 2592-2599 (2014).

17. Napolitano, A.P. et al. Scaffold-free three-dimensional cell culture utilizing micromolded nonadhesive hydrogels. BioTechniques 43, 494, 496-500 (2007).

18. Rago, A.P., Dean, D.M. \& Morgan, J.R. Controlling cell position in complex heterotypic 3D microtissues by tissue fusion. Biotechnology and bioengineering 102, 1231-1241 (2009).

19. Bratt-Leal, A.M., Kepple, K.L., Carpenedo, R.L., Cooke, M.T. \& McDevitt, T.C. Magnetic manipulation and spatial patterning of multi-cellular stem cell aggregates. Integrative biology : quantitative biosciences from nano to macro 3, 1224-1232 (2011).

20. Chen, P., Guven, S., Usta, O.B., Yarmush, M.L. \& Demirci, U. Biotunable acoustic node assembly of organoids. Advanced healthcare materials 4, 1937-1943 (2015).

21. Gjorevski, N., Boghaert, E. \& Nelson, C.M. Regulation of Epithelial-Mesenchymal Transition by Transmission of Mechanical Stress through Epithelial Tissues. Cancer microenvironment : official journal of the International Cancer Microenvironment Society 5, 29-38 (2012).

22. Cheng, S.L., Yang, J.W., Rifas, L., Zhang, S.F. \& Avioli, L.V. Differentiation of human bone marrow osteogenic stromal cells in vitro: induction of the osteoblast phenotype by dexamethasone. Endocrinology 134, 277-286 (1994).

23. Cohen, D.E. \& Melton, D. Turning straw into gold: directing cell fate for regenerative medicine. Nature reviews. Genetics 12, 243-252 (2011).

24. Li, W., Jiang, K., Wei, W., Shi, Y. \& Ding, S. Chemical approaches to studying stem cell biology. Cell research 23, 81-91 (2013).

25. Artus, J., Piliszek, A. \& Hadjantonakis, A.K. The primitive endoderm lineage of the mouse blastocyst: sequential transcription factor activation and regulation of differentiation by Sox17. Developmental biology 350, 393-404 (2011).

26. Ying, Q.L. et al. The ground state of embryonic stem cell self-renewal. Nature 453, 519-523 (2008).

27. Stavridis, M.P., Collins, B.J. \& Storey, K.G. Retinoic acid orchestrates fibroblast growth factor signalling to drive embryonic stem cell differentiation. Development 137, 881-890 (2010).

28. Cho, L.T. et al. Conversion from mouse embryonic to extra-embryonic endoderm stem cells reveals distinct differentiation capacities of pluripotent stem cell states. Development 139, 2866-2877 (2012).

29. Plusa, B., Piliszek, A., Frankenberg, S., Artus, J. \& Hadjantonakis, A.K. Distinct sequential cell behaviours direct primitive endoderm formation in the mouse blastocyst. Development 135, 30813091 (2008).

30. Jakab, K., Neagu, A., Mironov, V., Markwald, R.R. \& Forgacs, G. Engineering biological structures of prescribed shape using self-assembling multicellular systems. Proceedings of the National Academy of Sciences of the United States of America 101, 2864-2869 (2004).

31. Levenberg, S. et al. Engineering vascularized skeletal muscle tissue. Nature biotechnology 23, 879-884 (2005).

32. Rouwkema, J., de Boer, J. \& Van Blitterswijk, C.A. Endothelial cells assemble into a 3-dimensional prevascular network in a bone tissue engineering construct. Tissue engineering 12, 2685-2693 (2006).

33. Rouwkema, J., Rivron, N.C. \& van Blitterswijk, C.A. Vascularization in tissue engineering. Trends Biotechnol 26, 434-441 (2008).

34. Rivron, N.C. et al. Sonic Hedgehog-activated engineered blood vessels enhance bone tissue formation. Proceedings of the National Academy of Sciences of the United States of America 109, 4413-4418 (2012).

35. Heisenberg, C.P. \& Bellaiche, Y. Forces in tissue morphogenesis and patterning. Cell 153, 948-962 (2013).

36. Keller, R. Developmental biology. Physical biology returns to morphogenesis. Science 338, 201-203 (2012).

37. McBeath, R., Pirone, D.M., Nelson, C.M., Bhadriraju, K. \& Chen, C.S. Cell shape, cytoskeletal tension, and RhoA regulate stem cell lineage commitment. Developmental cell 6, 483-495 (2004).

38. Beckermann, B.M. et al. VEGF expression by mesenchymal stem cells contributes to angiogenesis in pancreatic carcinoma. Br J Cancer 99, 622-631 (2008).

39. Zisa, D., Shabbir, A., Suzuki, G. \& Lee, T. Vascular endothelial growth factor (VEGF) as a key therapeutic trophic factor in bone marrow mesenchymal stem cell-mediated cardiac repair. Biochem Biophys Res Commun 390, 834-838 (2009). 
40. Gerhardt, H. VEGF and endothelial guidance in angiogenic sprouting. Organogenesis 4, 241-246 (2008).

41. Siekmann, A.F., Covassin, L. \& Lawson, N.D. Modulation of VEGF signalling output by the Notch pathway. BioEssays : news and reviews in molecular, cellular and developmental biology 30, 303-313 (2008).

42. Vempati, P., Popel, A.S. \& Mac Gabhann, F. Extracellular regulation of VEGF: isoforms, proteolysis, and vascular patterning. Cytokine \& growth factor reviews 25, 1-19 (2014).

43. Beerepoot, L.V., Shima, D.T., Kuroki, M., Yeo, K.T. \& Voest, E.E. Up-regulation of vascular endothelial growth factor production by iron chelators. Cancer research 56, 3747-3751 (1996).

44. Robinson, C.J. \& Stringer, S.E. The splice variants of vascular endothelial growth factor (VEGF) and their receptors. Journal of cell science 114, 853-865 (2001).

45. Giacca, M. Non-redundant functions of the protein isoforms arising from alternative splicing of the VEGF-A pre-mRNA. Transcription 1, 149-153 (2010).

46. Martino, M.M. et al. Growth factors engineered for super-affinity to the extracellular matrix enhance tissue healing. Science 343, 885-888 (2014).

47. Chen, T.T. et al. Anchorage of VEGF to the extracellular matrix conveys differential signaling responses to endothelial cells. The Journal of cell biology 188, 595-609 (2010).

48. Tung, Y.C. et al. High-throughput 3D spheroid culture and drug testing using a 384 hanging drop array. The Analyst 136, 473-478 (2011).

49. Gottwald, E. et al. A chip-based platform for the in vitro generation of tissues in three-dimensional organization. Lab on a chip 7, 777-785 (2007).

50. Sridhar, A., de Boer, H.L., van den Berg, A. \& Le Gac, S. Microstamped Petri dishes for scanning electrochemical microscopy analysis of arrays of microtissues. PloS one 9, e93618 (2014).

51. Altmann, B. et al. Microstructuring of multiwell plates for three-dimensional cell culture applications by ultrasonic embossing. Biomedical microdevices 14, 291-301 (2012).

52. Desroches, B.R. et al. Functional scaffold-free 3-D cardiac microtissues: a novel model for the investigation of heart cells. American journal of physiology. Heart and circulatory physiology 302 , H2031-2042 (2012).

53. Ye, X. et al. Scalable units for building cardiac tissue. Adv Mater 26, 7202-7208 (2014).

54. Sekine, H. et al. In vitro fabrication of functional three-dimensional tissues with perfusable blood vessels. Nature communications 4, 1399 (2013).

55. Folkman, J. Tumor angiogenesis: therapeutic implications. N Engl J Med 285, 1182-1186 (1971).

56. Zhou, J., Kim, H.Y. \& Davidson, L.A. Actomyosin stiffens the vertebrate embryo during crucial stages of elongation and neural tube closure. Development 136, 677-688 (2009).

57. Olsson, A.K., Dimberg, A., Kreuger, J. \& Claesson-Welsh, L. VEGF receptor signalling - in control of vascular function. Nature reviews. Molecular cell biology 7, 359-371 (2006).

58. Vander Heiden, M.G., Cantley, L.C. \& Thompson, C.B. Understanding the Warburg effect: the metabolic requirements of cell proliferation. Science 324, 1029-1033 (2009).

59. Miquerol, L., Langille, B.L. \& Nagy, A. Embryonic development is disrupted by modest increases in vascular endothelial growth factor gene expression. Development 127, 3941-3946 (2000).

60. Kamba, T. et al. VEGF-dependent plasticity of fenestrated capillaries in the normal adult microvasculature. American journal of physiology. Heart and circulatory physiology 290, H560-576 (2006).

61. Banai, S. et al. Upregulation of vascular endothelial growth factor expression induced by myocardial ischaemia: implications for coronary angiogenesis. Cardiovasc Res 28, 1176-1179 (1994).

62. Siddappa, R. et al. cAMP/PKA pathway activation in human mesenchymal stem cells in vitro results in robust bone formation in vivo. Proceedings of the National Academy of Sciences of the United States of America 105, 7281-7286 (2008).

63. Lamprecht, M.R., Sabatini, D.M. \& Carpenter, A.E. CellProfiler: free, versatile software for automated biological image analysis. BioTechniques 42, 71-75 (2007).

64. Xia, Y. et al. Complex Optical Surfaces Formed by Replica Molding Against Elastomeric Masters. Science 273, 347-349 (1996). 


\section{Chapter 3}

\section{D High Throughput Screening and Monitoring of Embryoid Bodies in Thermoformed Microwell Plates}

E.J. Vrij, ${ }^{1,2}$, S. Espinoza ${ }^{1,5}$, M. Heilig ${ }^{3}$, A. Kolew ${ }^{3}$, A.M. Schneider ${ }^{3}$, C.A. van Blitterswijk ${ }^{1,2}$, R. Truckenmüller $^{1,2}$, N.C. Rivron ${ }^{1,2,4}$

1. MIRA -Institute for Biomedical Technology and Technical Medicine- University of Twente, Enschede, The Netherlands.

2. MerIn Institute for Technology-Inspired Regenerative Medicine, Maastricht University, Maastricht, The Netherlands.

3. Institute of Microstructure Technology, Karlsruhe Institute of Technology (KIT), Eggenstein-Leopoldshafen, Germany

4. Hubrecht Institute for developmental biology and stem cells research, Utrecht, The Netherlands

5. Fachbereich Physikalische Technik, Münster University of Applied Sciences, Steinfurt, Germany 
Chapter 3 


\section{Abstract}

3D organoids using stem cells to study development and disease are now widespread. These models are powerful to mimic in vivo situations but are currently associated with high variability and low throughput. For biomedical research, platforms are thus necessary to increase reproducibility and allow High-Throughput Screens (HTS). Here, we introduce a microwell platform, integrated in standard culture plates, for functional HTS. Using micro-thermoforming, we form round-bottom microwell arrays from optically clear cyclic olefin polymer films, and assemble them with bottomless 96-well plates. We show that embryonic stem cells aggregate faster and more reproducibly (centricity, circularity) as compared to a state-of-the-art microwell array. We then run a screen of a chemical library to direct differentiation into primitive endoderm ( $\operatorname{PrE}$ ) and, using on-chip High Content Imaging $(\mathrm{HCl})$, we identify molecules, including regulators of the cAMP pathway, regulating tissue size, morphology and PrE gene activity. We propose that this platform will benefit to the systematic study of organogenesis in vitro.

Keywords: Spheroids, embryoid bodies, microwells, micro-thermoforming, high-throughput screening, high-content imaging, directed differentiation, primitive endoderm 


\section{Introduction}

In vivo, cells reside within 3D environments, which create specific interactions that are not necessarily replicated in conventional (2D) cell culture. However, many cell types aggregate into 3D clusters, which better recapitulate structural organizations and biological functions. ${ }^{1}$ Embryonic Stem (ES) cells aggregate into Embryoid Bodies (EBs) with the intrinsic capacity to recapitulate development and form organ models in vitro, including the gut, ${ }^{2}$ the eye ${ }^{3}$ and the brain. ${ }^{4,5}$ These organoids form upon sequential exposure to combinations of soluble factors that orchestrate proliferation, differentiation and morphogenesis. Such models are important to understand organ development and disease and, in the context of drug screens, as models of intermediate complexity between 2D culture and animal models. ${ }^{6,7}$ However, they are currently produced at a low throughput and with a low efficiency. There is thus a need for platforms increasing control and throughput while allowing direct on-chip measurements.

EBs are classically and still widely formed using the hanging drop method or round-bottom 96 well plates (96wps). Evolutions of these culture techniques aim at decreasing the complexity of manipulations while increasing the reproducibility and throughput. In that context, laboratories working at the interface of microfabrication and cell-biology developed non-adhesive microwell arrays to spatially confine small, defined numbers of cells and control aggregation. Microwell arrays can be formed by hot embossing of polystyrene, ${ }^{8,9}$ replica molding of polydimethylsiloxane ${ }^{10,11}$ or hydrogels ${ }^{12-14}$ or micro-thermoforming. ${ }^{15}$ Of special interest for organoid systems, microwell arrays also preserve control over culture conditions (medium refreshment, substrate interaction, sequential addition of soluble factors) while allowing for active monitoring over increased number of replicates. Microwell platforms require high resolution microwells, high number of microwells per well, high number of conditions per plate, uniform cell seeding, easy harvesting of aggregates, compatibility with liquid handling systems and automated microscopes, and potential for manufacturing.

Here, we propose a micro-thermoformed microwell platform for the formation, screening and automated imaging of organoids. Micro-thermoforming of thermoplastic films ${ }^{16}$ is a method applicable to numerous materials, which bridges length scales and allows surface pre-modifications. 17-19 An important benefit of micro-thermoforming over, for example, hot embossing is that it permits large-area high-precision forming of microwells with a very thin bottom (below 10 micrometers), thus enabling reproducible, high-magnification microscopy. The platform we present is based on a standard 96wp to maximize the compatibility with established instruments, such as automated liquid-handling and imaging systems. We validated its potential for HTS using a library of small molecules classified as kinase inhibitors. Via $\mathrm{HCl}$, we extracted features of cellular proliferation, gene activity, tissue size and morphology and assessed the functions of these small molecules in the context of primitive endoderm differentiation. 


\section{Results}

\section{Fabrication of thermoformed microwell screening plate}

The platform consists of a bottomless 96wp mounted with a thermoformed Cyclic Olefin coPolymer (COP) film $(50 \mu \mathrm{m})$ imprinted with 96 microwell arrays. COP is a thermoplastic material with superior optical properties for fluorescent imaging as compared to polystyrene. It has low turbidity (haze value $<0.1 \%$ ) and low wavelength dispersion in the visible and near-UV spectrum. Additionally, the non-polar surface minimizes cellular adhesion. ${ }^{20}$ Microwells were microthermoformed all at once by a negative free-forming process. COP films were heated up to their rubber-elastic state by contact with the mold and then stretched into the evacuated mold recesses by pressurized nitrogen (Figure $1 \mathrm{~A}$ ). Depending on the COP material and the location on the plate this yielded microwells with a depth of approximately $260 \mu \mathrm{m}$ (Figure 1B) and an inner diameter of about $300 \mu \mathrm{m}$ (Figure 1C). The microwells are hemispherical, which should improve cell aggregation.

The thermoformed films had lateral dimensions equivalent to that of a full 96wp. They were aligned and pressure bonded to bottomless $96 \mathrm{wps}$ by inserting a cytocompatible double-sided tape (acrylic elastomer, Figure 1D). The 96wp, the film and the tape, with punched holes aligned with the wells of the plate, constitute the full platform. Each well of the 96wp contains an array of 144 microwells constituted in a $12 \times 12$ grid aligned to the center of the well (Figure 1E). After sterilization with ethanol, the platform was used by simply dispensing a cell suspension into the wells of the $96 \mathrm{wp}$.

a

Micro-thermoforming process
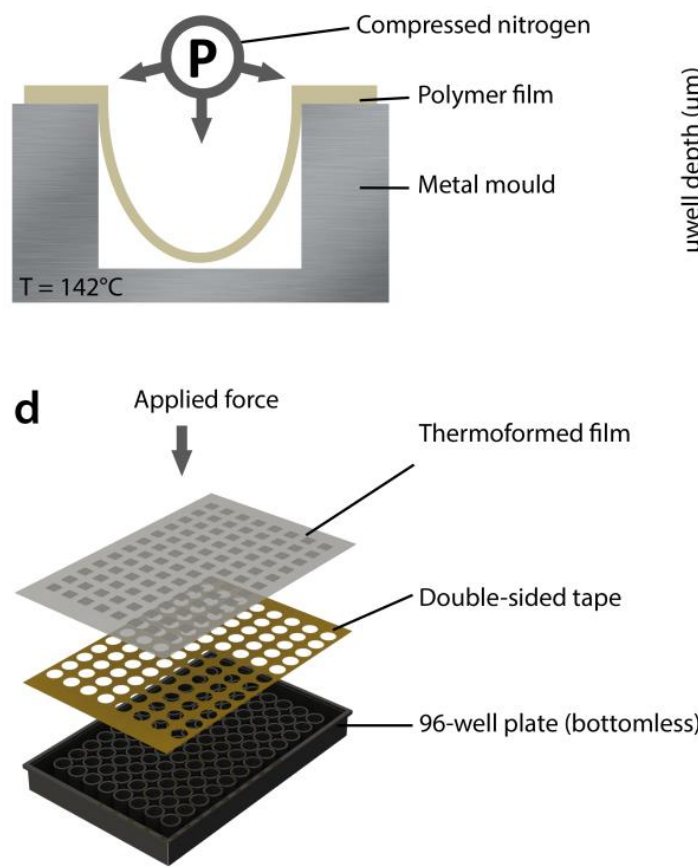

b

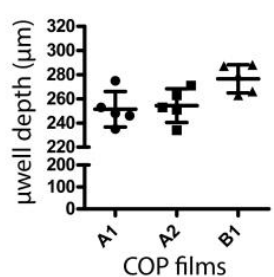

C

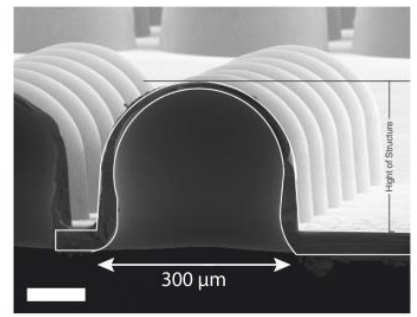

e

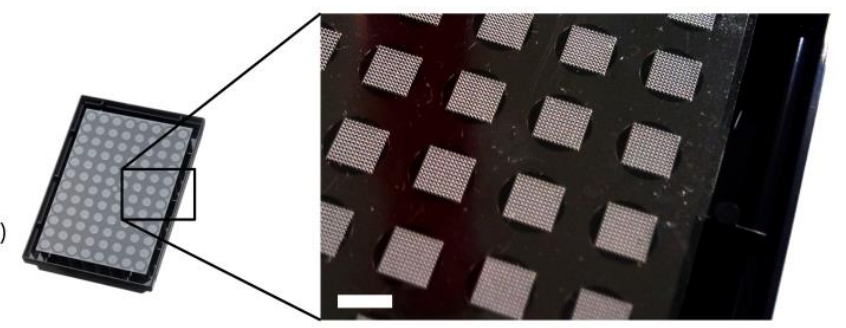

Figure 1: Fabrication method of thermoformed microwell screening plate. (A) The microwells are fabricated in a $50 \mu \mathrm{m}$ thin COP film through a process of micro negative free-(thermo)forming. Thereby, the heated and softened film is formed into a mold of circular microcavities under high pressure. (B) Micro-thermoforming depth was measured at multiple locations on different COP films. The depth was measured at 5 different 
locations on the plate. (C) Scanning electron microscopy image of a microwell section depicting its hemispherical geometry. The microwells have a diameter of around $300 \mu \mathrm{m}$ and a depth of approximately 260 $\mu \mathrm{m}$. Scale bar: $100 \mu \mathrm{m}$. (D) The micro-thermoformed film was pressure-bonded to the bottomless 96wp through a punched cytocompatible double-sided tape. (E) Image of the backside of the full thermoformed microwell screening plate, including an enlarged image showing the microwell arrays. Each array corresponds with a single well of the 96wp. Scale bar: $5 \mathrm{~mm}$.

\section{ESC aggregation kinetics}

COP is described as a low-adherent material for cells. ${ }^{20}$ When mouse ES cells were seeded within the microwells, they spontaneously aggregated into EBs. However, partial cell adhesion affected the circularity and centricity of EBs (Figure 2A negative control). To further reduce cell adhesion and improve formation of EBs, we pre-treated the film with a solution of Pluronics F108. Centricity and circularity of the aggregate increased with increasing concentrations of Pluronics F108 (Figure 2A). Because differences in centricity and circularity were low between $1 \%$ and $5 \% \mathrm{w} / \mathrm{v}$ Pluronics (Figure $2 \mathrm{~A}$ ), we chose $1 \%$ as a standard concentration in further experiments.

We compared the round-bottom microwell array, with our state-of-the-art flat-bottom hydrogel microwell array. ${ }^{14}$ The geometry of the thermoformed microwells rapidly concentrated cells in the center, which formed a single aggregate within 1 hour (Figure 2B, C). In contrast, cells in the flatbottom microwells formed a single aggregate within 6 to 11 hours (Figure 2B, C). In addition, EBs centricity and circularity within microwells increased more rapidly in the round-bottom microwells as compared to the flat-bottom microwells (Figure 2A, D). Within 24 hours EBs in the round-bottom microwells had an average circularity of 0.9 (Figure 2D) and their size increased from 11 hours of culture onward (Figure 2E), which suggested cellular proliferation.

After 48 hours of culture, the number of dead cells was similarly low between the two microwell arrays as assessed with calcein AM and ethidium homodimer (Figure 2F). As a control, we added glue extract from the tape directly to the medium, which did not increase cell death (Figure 2F). We concluded that the bonding process and the glue did not affect cell viability.

Overall, the data demonstrated that the thermoformed microwell array increased the efficiency of ES cell aggregation (speed, centricity, circularity) as compared to our standard array. 
a
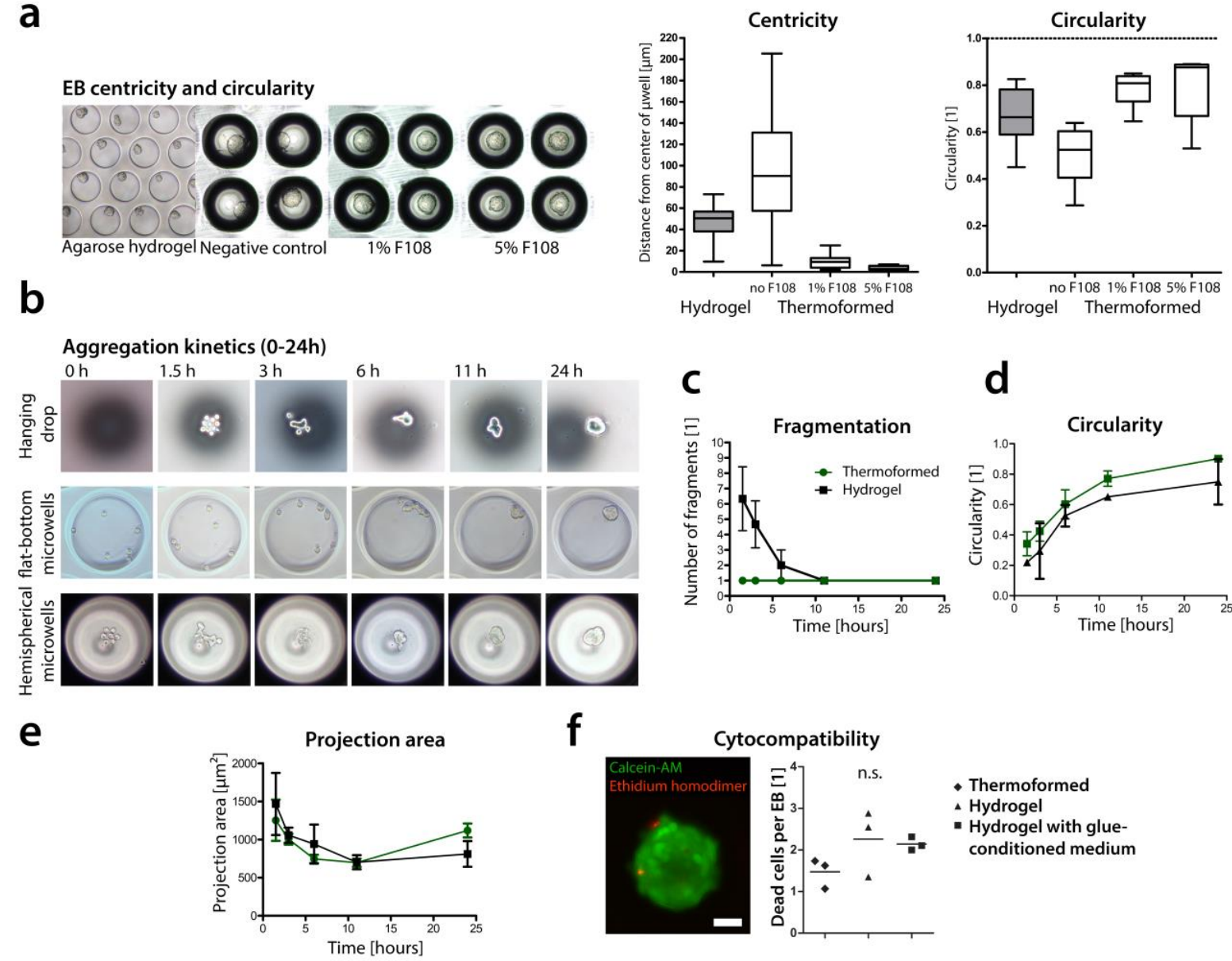

d

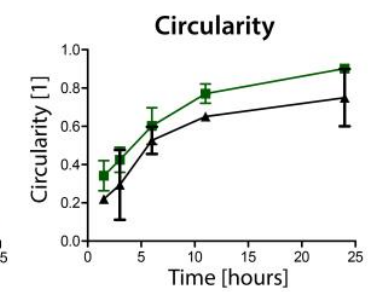

Figure 2: Embryonic stem cell aggregation kinetics. (A) Thermoformed COP films were pre-treated with 0 (negative control), 1 and 5\% w/v Pluronics F108 in demi-water for 1 hour at $37^{\circ} \mathrm{C}$. EBs from 100 cells per microwell formed within 24 hours of culture. Box-and-whiskers plots displaying the maxima and minima (whiskers) and the median with quartiles (boxes) for the centricity (distance between center of EB and center of microwell) and circularity of EBs ( $n=5$ wells). (B) EB aggregation kinetics in thermoformed and hydrogel microwells and in hanging drops displayed in a time series of bright field images. (C) Number of cellular fragments, (D) circularity of the biggest fragments, and (E) total projection area of fragments measured over time ( $n=3$ wells). (F) Viability assay on ES cells using calcein-AM and ethidium homodimer shows dead cells in red and viable cells in green $(n \geq 12)$. 50 cells per microwell were seeded and cultured for 48 hours before stained and imaged. Scale bar: $50 \mu \mathrm{m}$. No statistically significant difference was observed between the conditions (ANOVA), suggesting no toxic effects from the glue.

\section{Embryoid body culture}

Obtaining proper statistics in the context of 3D development necessitates a uniform seeding distribution of cells over a high number of replicates. We assessed the distribution of cells within microwells by seeding a target number of 50 or 100 cells per microwell. At 72 hours, the distribution of the projection area of EBs (area seen from the top, used as a proxy for EB volume) deviated $12 \%$ from the median for 100 cells and $21 \%$ for 50 cells (Figure $3 A$ ). Results were similar between the two arrays, during the first 48 hours (Figure 3B). Over time, we observed a drastic increase in EBs size on the thermoformed array (Figure 3B). Conversely, the flat-bottom hydrogel microwells, having a smaller diameter $(200 \mu \mathrm{m})$, showed a plateau for EB size at approximately $50 \%$ of the projection area of the microwell. The on-chip measurement of the number of cells per microwell is an 
important feature for such a platform. Here, we observed that the thermoformed array differs from the hydrogel array by allowing on-chip trypsinization and subsequent counting of the number of cells per microwell. Such an on-chip trypsinization and counting assay showed high correlation between the projection area and the number of cells (Figure 3C).

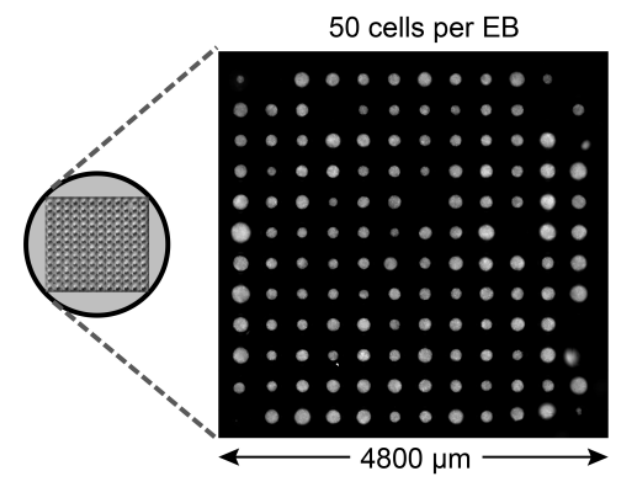

b

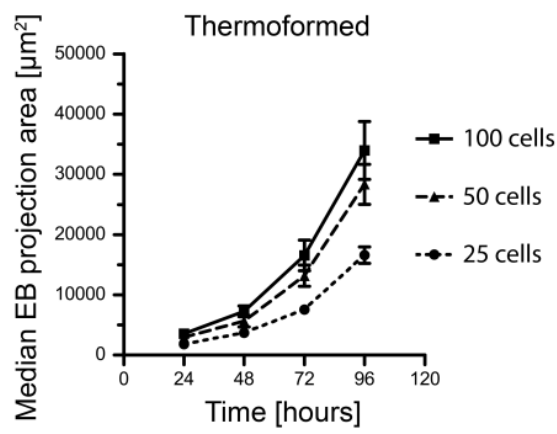

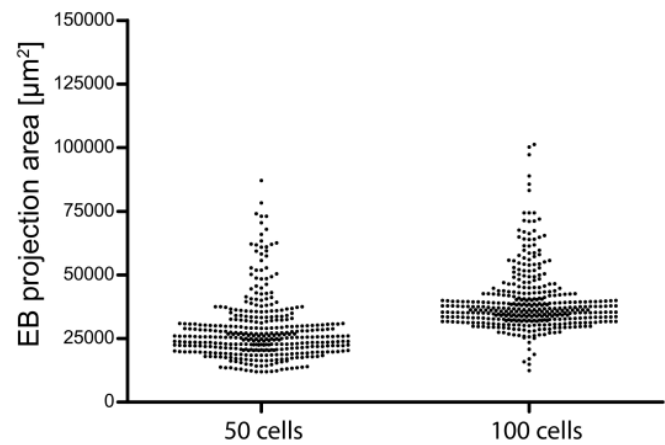

C

Figure 3: Embryoid body culture. (A) EBs were cultured for 72 hours, captured using montage imaging, and analyzed using Cell Profiler software ${ }^{21}$. The distribution of EB size was plotted for 3 pooled wells, each containing $>100$ EBs. (B) Projection area of EBs as a function of time ( $n=6$ wells, median was taken among $>80$ and $>200$ EBs per well from hydrogel and thermoformed plates, respectively). For comparison, $31400 \mu \mathrm{m}^{2}$ and $\sim 70.000 \mu \mathrm{m}^{2}$ equals the projection area of a hydrogel and a thermoformed microwell, respectively. (C) Average number of cells per EB counted after on-chip trypsinization. Data points from different time points $(24,48$ and 72 hours) and cell seeding densities (50 and 100 cells per microwell) were plotted together and fitted by a power function, $\mathrm{n}=3$ wells per data point.

\section{Screening EBs for PDGFR $\alpha$-H2B-GFP expression using a kinase}

\section{inhibitor library}

Primitive endoderm (PrE) is the first tissue arising from pluripotent stem cells within the inner cell mass of the blastocyst and within EBs. Here, we used this model of differentiation to test the potential of the platform for $\mathrm{HTS}$ and $\mathrm{HCl}$. We used an embryonic stem cell line containing a fluorescent nuclear reporter for the genetic activity of the PrE marker PDGFR $\alpha$ (PDGFR $\alpha$-H2B-GFP). ${ }^{22,23}$ We formed EBs by seeding a target number of 13 ES cells per microwell. After aggregation (24 hours), we administered a kinase inhibitor library ( 80 compounds) both at 24 and 72 hours. After 96 hours, we fixed the EBs and assessed differentiation via on-chip imaging (Figure 4A). We quantified 
the yield of fluorescent reporter expression as the percentage of EBs positive for PDGFR $\alpha$-H2B-GFP (Figure 4B). PDGFR $\alpha-H 2 B-G F P^{+}$cells were mostly located in the periphery of EBs as observed by onchip epifluorescence imaging (Figure 4C) and on-chip confocal imaging (Figure 4D). In addition, we quantified morphological parameters, such as the projection area (Figure 4B) and the form-factor (Figure $4 \mathrm{E}$ ), as proxies for size and circularity, respectively. The compounds that were lethal to cells or autofluorescent were omitted from the analysis (7 compounds). We used Retinoic acid (RA) as a positive control inducing PrE differentiation. ${ }^{24}$ We used the combination of PD325901 (MEK inhibitor) and CHIR-99021 (GSK3-beta kinase inhibitor) as a negative control, due to its assessed function in inhibiting ES cell differentiation. ${ }^{25}$ As expected, Indirubin and Indirubin-3'-monoxime, which also target GSK3-beta kinase, had effects similar to the negative control along the 3 parameters (PDGFR $\alpha-H 2 B-G F P$ expression, projection area and form-factor) (Figure 4B, D), thus confirming the validity of the assay. The screen revealed that the small molecule Palmitoyl-DLcarnitine up-regulates PDGFR $\alpha$ activity. Palmitoyl-DL-carnitine is classified as a Protein Kinase C inhibitor, with recurrent function in epithelial transformation, which is essential for PrE differentiation ${ }^{26}$. Surprisingly, the small molecule AG-370, which was previously classified as a PDGFR $\alpha$ kinase inhibitor, acted as a PDGFR $\alpha$ activator in the context of PrE formation. 


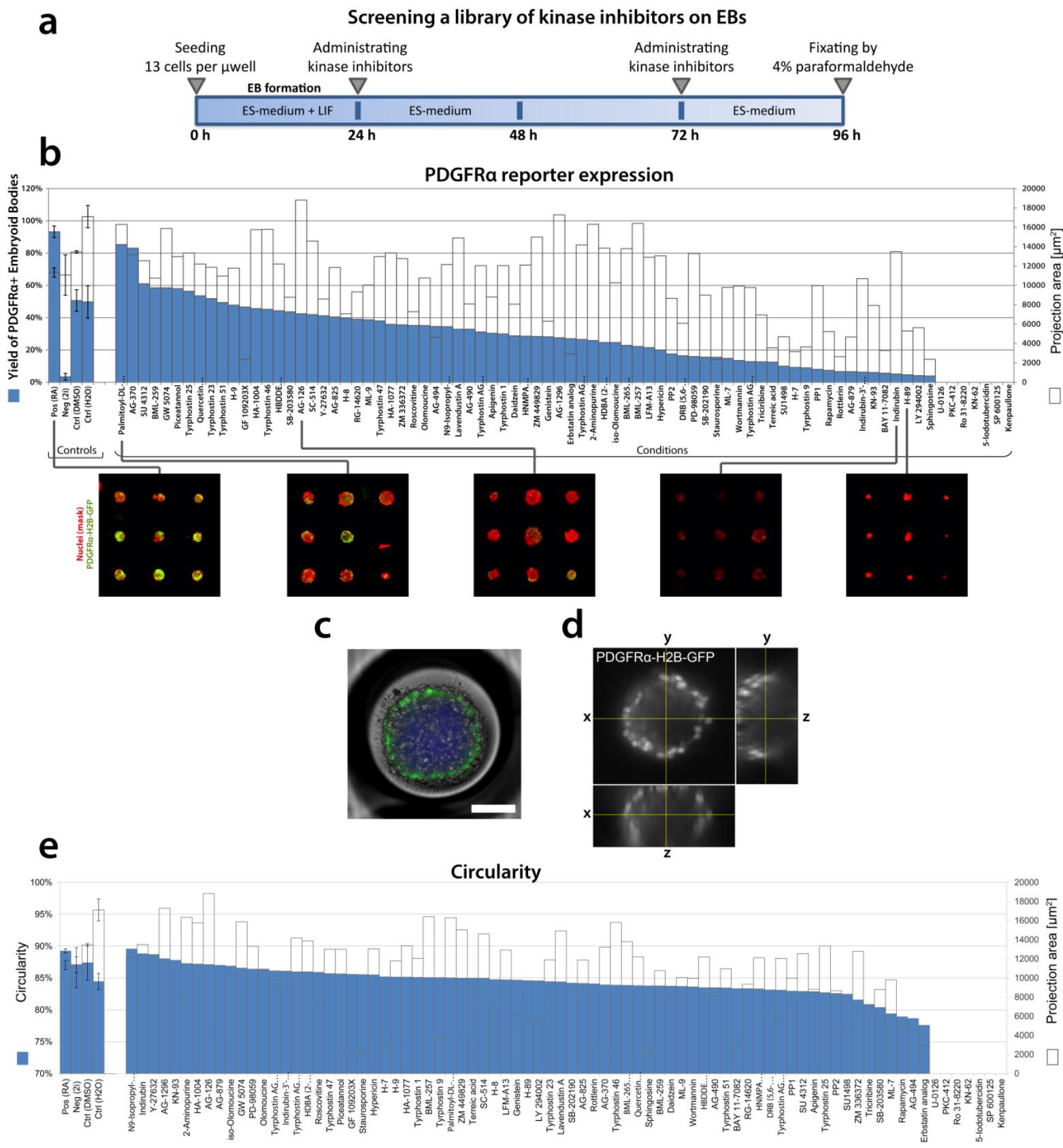

Figure 4: Kinase inhibitor screening on PDGFRa-H2B-GFP expression in EBs. (A) Schematic depicting screening. (B) Yield of EBs positive for PDGFR $\alpha-H 2 B-G F P$ expression sorted from high to low (blue bars) with the $E B$ size (white bars) including a series of representative fluorescent images ( $n=3$ wells for controls). (C) Overlay of bright field and fluorescent image of a single EB positive for PDGFR $\alpha-\mathrm{H} 2 \mathrm{~B}-\mathrm{GFP}$ (20x, blue: DAPI, green: PDGFR $\alpha-H 2 B-G F P)$. Scale bar: $100 \mu \mathrm{m}$. (D) Orthogonal images acquired via spinning disk confocal microscopy of a single EB including PDGFR $\alpha-\mathrm{H}_{2} \mathrm{~B}-\mathrm{GFP}^{+}$cells $(1 \mu \mathrm{m}$ interspacing using a 20x objective on BD Pathway 435). (E) Circularity of EBs sorted from high to low (blue bars) with the EB size (white bars). 


\section{Kinase classes}

We further analyzed the data by classifying compounds based on their reported kinase class and along the 3 features (Figure $5 \mathrm{~A}-\mathrm{C}$ ). Figure 5 shows that compounds with the same predicted kinaseclass target clearly have different effects (Figure 5D). For example, molecules classified as PDGFR $\alpha$ inhibitors either up-regulated or down-regulated PDGFR $\alpha$ activity in EBs (Figure 5A). Similar effects were observed with compounds classified as PKA, PKC and PI3K inhibitors. This observation correlates with studies pointing at the context-dependent and off-target effects of small chemicals ${ }^{27}$ and thus emphasizes the importance of cell type-specific HTS assays. In addition, our classification shows that molecules with the same predicted kinase-class target have similar effects on PDGFR $\alpha$ activity but dissimilar effects on EB size. We propose that this range of phenotypes reflects different biological functions such as the induction of ES cells differentiation into PDGFR $\alpha^{+}$cells or of proliferation of PDGFR $\alpha+$ cells. Multifactorial on-chip analysis, combining proliferation, morphological and genetic activity might thus yield new insights on the context-dependent functions of small molecules. Overall, these results show that the platform allows for HTS and the rapid monitoring of multiple parameters on-chip via automated $\mathrm{HCl}$.
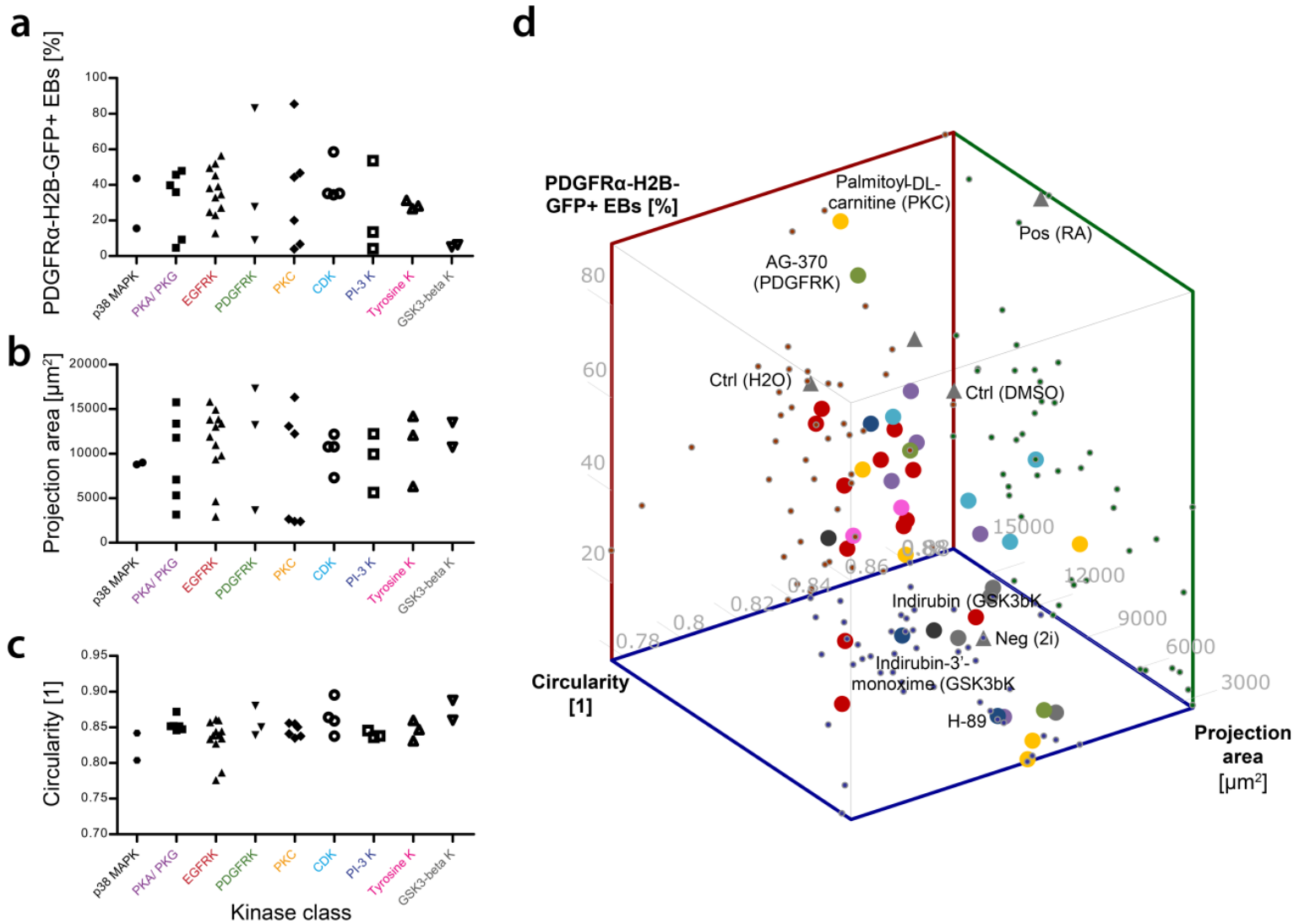

Figure 5: Kinase inhibitor screening analysis. (A) PDGFR $\alpha-H 2 B-G F P$ expression, (B) projection area of EBs, and (C) circularity of EBs sorted by the most prevalent kinase classes in the library. Depicted in green and red are the pathways currently known to regulate PrE. Depicted in purple and yellow are the novel pathways found to regulate PrE. (D) 3D graph combining data from the previous three graphs and including controls. 
Negative regulation of the CAMP/PKA pathway, specifically by small molecule $\mathrm{H}-89$, showed inhibition of PDGFR $\alpha$ in EBs (Figure 4B and 5A). This finding let us to the hypothesis that activation of the CAMP/PKA pathway might increase PDGFR $\alpha$ expression in EBs. To test this hypothesis, we designed an assay including the small molecules Forskolin, an upstream activator of cAMP/PKA signaling, and 8Br-cAMP, a cell-permeable analog of cAMP. Shown in Figure 6, both compounds significantly increased the yield of PDGFR $\alpha$-H2B-GFP positive EBs (more than 1.6 fold), hereby validating CAMP/PKA signalling as being a novel positive regulator of PDGFR $\alpha$ expression in EBs (Figure 6).

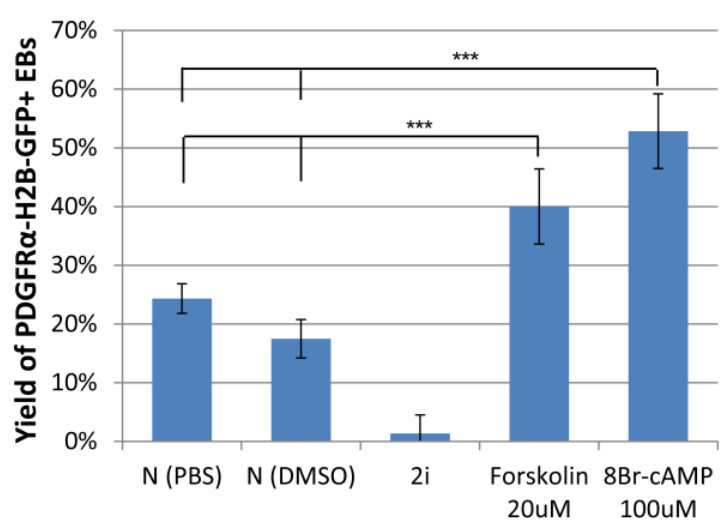

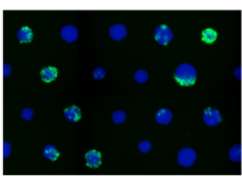

$8 \mathrm{Br}-\mathrm{CAMP} 100 \mu \mathrm{M}$

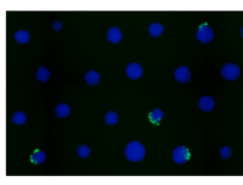

Neutral (PBS)

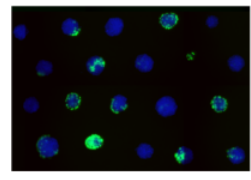

Forskolin $20 \mu \mathrm{M}$

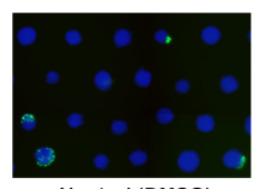

Neutral (DMSO)

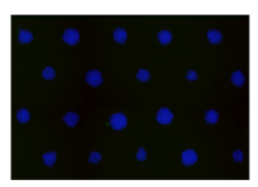

Negative (2i)

Figure 6: Activators of CAMP/PKA signaling 8Br-CAMP and Forskolin show an upregulation of EBs positive for PDGFRa-H2B-GFP compared to PBS and DMSO controls ( $n=6$ wells, ${ }^{* * *}$ denotes $p<0.001$, ANOVA with Tukey's multiple comparison test).

\section{Discussion}

Using a novel microwell platform, we described the effect of a panel of small molecule kinase inhibitors on EB size, form and gene activity. We describe two small molecules that acted in the primary screen as positive regulators for the reporter gene PDGFR $\alpha$ in the context of $\operatorname{PrE}$ differentiation. Negative regulation of the CAMP/PKA pathway, specifically by small molecule $\mathrm{H}-89$, showed inhibition of PDGFR $\alpha$ in EBs. This led us to validate activators of the CAMP/PKA pathway as PDGFR $\alpha$ is a gene involved in early embryonic differentiation of the PrE, from which the visceral yolk sac endoderm develops. The yolk sac is the primary site of hematopoiesis. ${ }^{28}$ As such, the 3D directed differentiation of EBs into PrE might serve as a starting point, for example, for the development of blood cells precursors. ${ }^{29,30}$ More generally, we speculate that platforms allowing for the reproducible, controlled and rapid formation of multiple replicates along with HTS will accelerate the use of organoids as models with greater prediction power for scientific and clinical studies.

In our system, however, the formed EBs are comparatively small to benefit larger organoid models. Also, on-chip imaging problems might occur for cells located in the periphery of biological models that approach the size of the microwells due to internal reflection of light within the curved polymer film. Therefore, developing a range of larger microwells (optionally with a flatter bottom) could aid the imaging quality and support the culture of larger organoid models, such as the kidney.

${ }^{31}$ To preserve a high number of microwells and to augment cell seeding uniformity even further, the squared array of microwells should be extended to cover the full surface area of the wells. For this 
purpose, costly new metal molds have to be micro-machined, which necessitates a thorough examination of useful sizes.

In micro-thermoforming a thin thermoplastic polymer film is formed within its softened, but still solid, thermoelastic state, which allows for the preservation of previously generated (surface and bulk) modifications of the film, such as topographical imprints of micro- and nanoscale, ${ }^{32}$ and localized cell adhesion domains through patterned UV-irradiation. ${ }^{18,19}$ Accordingly, the microwell screening plate can be tailored with desirable features by pre-modification of the polymer film. As was previously reported, thermoplastic films can be ion track-etched to yield micropores within microwells. ${ }^{18,33}$ Porous microwells, then in a 3D-Boyden-chamber or Transwell ${ }^{\circledR}$ approach, can potentially function as a biomimetic interface that allows soluble factors to be exchanged, which could be useful to study paracrine signaling between different tissues. We propose that microthermoforming technology opens new possibilities for the creation of more controlled and complex in vitro microenvironments.

\section{Conclusions}

We showed a microwell platform, fabricated by assembling micro-thermoformed films with standard 96-well culture plates, whose format is compatible with $\mathrm{HTS}$ and $\mathrm{HCl}$ systems. The platform increases the controlled manifold formation and chemical exposure of EBs along with the acquisition of features directly on-chip, such as cellular proliferation via direct trypsinization/counting and aggregate morphology and gene activity via automated microscopy. We validated the platform for HTS using a library of small molecule kinase inhibitors and observed the functional specificity of small molecules in the context of PrE differentiation. The finding of CAMP/PKA as a novel pathway in PrE differentiation shows that this platform is suitable for the unbiased identification of compounds directing tissue development. We propose that this approach will benefit to the systematic production of cells, tissues and organs with an impact in pharmacology and regenerative medicine.

\section{Materials and Methods}

\section{Thermoplastic films}

Cyclic olefin copolymer (COP) films had a thickness of $50 \mu \mathrm{m}$ and were obtained from two different suppliers: 'Zeonor mcs foil 051' films were obtained from microfluidic ChipShop GmbH and 'ZeonorFilm ZF14-050, Zeonex' films were obtained from Sidaplax. This polymer has a glass transition temperature of ca. $136^{\circ} \mathrm{C}$.

\section{Mold fabrication and microthermoforming}

The wellplate-sized brass mold containing the microholes was fabricated by mechanical micromachining (microdrilling). Then, the cyclic olefin polymer film was clamped between the brass mold and a brass counterplate. Microthermoforming was performed by heating the film followed by applying a differential pressure through an integrated channel within the counterplate, which 
stretched the film onto the micromachined mold. After cooling down the microthermoformed COP film was released.

\section{Assembly of the plate}

$3 \mathrm{M}$ VHB acrylic elastomer tape, a biocompatible double-sided tape, was punched with holes using a custom-made punching device. Then, this tape was used to adhere the COP film containing the microwell arrays to a bottom-less 96 -well plate. A force of about $30 \mathrm{~N}$ was applied at room temperature for 12 hours on top of the assembly for bonding. Sterilization was done by immersing the plate in $70 \% \mathrm{v} / \mathrm{v}$ ethanol in demi-water for 15 minutes. Full wetting of the microwells was achieved by shortly applying a vacuum.

\section{Cell repellence}

A solution of 0,1 and 5\% w/v of Pluronics F108 (Sigma-Aldrich) was prepared in sterile demiwater, dispensed into the thermoformed microwell array plate as $100 \mu \mathrm{L}$ per well and incubated overnight at $37^{\circ} \mathrm{C}$. The Pluronics solution was gently washed out from the wells using sterile demiwater before the cells were seeded into the microwells.

\section{Cell culture and reagents}

Mouse ES cells were expanded on a feeder layer of mouse embryonic fibroblasts (mEF) in ES medium containing: Dulbecco's Modified Eagle's Medium High Glucose (Life Technologies) supplemented with $10 \%$ fetal bovine serum (FBS, Greiner), $4 \mathrm{mM}$ Glutamax (Life Technologies), $100 \mathrm{U} / \mathrm{mL}$ penicillin (Life Technologies), $100 \mathrm{mg} / \mathrm{mL}$ streptomycin (Life Technologies), $10 \mathrm{mM}$ nonessential amino acids (Life Technologies), and freshly supplemented with $0.05 \mathrm{mM} 2$ mercaptoethanol (Life technologies), $1000 \mathrm{U} / \mathrm{mL}$ leukaemia inhibitory factor (LIF, Life Technologies), $3 \mu \mathrm{M}$ CHIR99021 (GSK3 $\beta$ inhibitor, Axon Medchem) and $1 \mu \mathrm{M}$ PD0325901 (MEK/ERK inhibitor, Sigma Aldrich). EBs were formed using a mouse ES cell line comprising a PDGFR ${ }^{\mathrm{H} 2 \mathrm{~B}-\mathrm{gfp} /+}$ knock-in allele (a histone H2B-GFP fusion protein reporter under the control of PDGFR $\alpha$ regulatory elements ${ }^{23}$ ).

\section{Kinase inhibitor screening}

A kinase inhibitor library V2.2 (80 chemicals reconstituted at $10 \mathrm{mM}$ in DMSO, Enzo Life Sciences) was used to screen for molecules that modulate H2B-GFP reporter activity in EBs formed using a mouse ES cell line comprising a PDGFR ${ }^{\mathrm{H} 2 \mathrm{~B}-\mathrm{gfp} /+}$ knock-in allele. A target number of $1872 \mathrm{ES}$ cells was seeded per well, which corresponds to 13 ES cells per microwell. ES cells were allowed to aggregate over 24 hours in $250 \mu \mathrm{L} /$ well of ES-medium including 10\% FBS (selected batch for ES culture, Greiner) and supplemented with $1 \mu \mathrm{g} / \mathrm{mL}$ LIF (LifeTechnologies) and $50 \mu \mathrm{M} \beta$ mercaptoethanol (Sigma-Aldrich). After 24 hours and 72 hours $200 \mu \mathrm{L}$ of medium was refreshed with ES medium including $10 \% \mathrm{FBS}$ and supplemented with $50 \mu \mathrm{M} \beta$-mercaptoethanol, and the kinase inhibitor compounds were administered in a working concentration of $20 \mu \mathrm{M}$. As a negative control $2 \mathrm{i}$ conditions were applied (combination of $1 \mu \mathrm{M}$ PD325901, $3 \mu \mathrm{M}$ CHIR-99021 and $1 \mu \mathrm{g} / \mathrm{mL}$ LIF). As a positive controls $1 \mu \mathrm{M}$ RA was used. During embryonic development the PrE is firmly established after 96 hours, ${ }^{23}$ thus after 96 hours of culture EBs were fixated using 4\% w/v paraformaldehyde, washed in PBS, stained with $100 \mu \mathrm{L}$ of $0.7 \mu \mathrm{g} / \mathrm{mL}$ DAPI solution (Sigma Aldrich, D9542), washed in PBS and imaged on-chip using the BD Pathway 435 and its image-montage function in combination with 
a 10x objective. Phenotypical data were acquired from montaged images using a custom-made pipeline in CellProfiler2.0 (Broad Institute). ${ }^{34}$

\section{Measurements}

For the ES cell aggregation kinetics the projection area, circularity and fragment number were measured using Image J (http://rsbweb.nih.gov/ij/). Circularity measured in Image J and CellProfiler (circularity equals formfactor) was calculated as $4 \pi^{*}[$ Area $] /\left([\text { Perimeter }]^{2}\right)$. Background intensity was removed from all images, except the PDGFR $\alpha$ images, by subtracting the average overall intensity from the whole image.

\section{Acknowledgements}

EJV gratefully acknowledge the Netherlands Institute for Regenerative Medicine program (NIRM). NCR gratefully acknowledge the Translational Adult Stem Cell program (ZonMW). This work was carried out with the technical support of the Karlsruhe Nano Micro Facility (KNMF, www.kit.edu/knmf), a Helmholtz Research Infrastructure at Karlsruhe Institute of Technology (KIT, www.kit.edu), and of European Research Infrastructure for multimaterial micro and nano fabrication and characterization (EUMINAfab). We also want to express our gratitude to Anna-Katerina Hadjantonakis for providing the PDGFRa ${ }^{\mathrm{H2B}-G F P /+}$ embryonic stem cell line.

\section{References}

1. Schwartz, M.A. \& Chen, C.S. Cell biology. Deconstructing dimensionality. Science 339, 402-404 (2013).

2. McCracken, K.W. et al. Modelling human development and disease in pluripotent stem-cell-derived gastric organoids. Nature 516, 400-404 (2014).

3. Eiraku, M. et al. Self-organizing optic-cup morphogenesis in three-dimensional culture. Nature 472, 51-56 (2011).

4. Lancaster, M.A. et al. Cerebral organoids model human brain development and microcephaly. Nature 501, 373-379 (2013).

5. Pasca, A.M. et al. Functional cortical neurons and astrocytes from human pluripotent stem cells in 3D culture. Nature methods 12, 671-678 (2015).

6. Shamir, E.R. \& Ewald, A.J. Three-dimensional organotypic culture: experimental models of mammalian biology and disease. Nature reviews. Molecular cell biology 15, 647-664 (2014).

7. van de Wetering, M. et al. Prospective derivation of a living organoid biobank of colorectal cancer patients. Cell 161, 933-945 (2015).

8. Sridhar, A., de Boer, H.L., van den Berg, A. \& Le Gac, S. Microstamped Petri dishes for scanning electrochemical microscopy analysis of arrays of microtissues. PloS one 9, e93618 (2014).

9. Altmann, B. et al. Microstructuring of multiwell plates for three-dimensional cell culture applications by ultrasonic embossing. Biomedical microdevices 14, 291-301 (2012).

10. Choi, Y.Y. et al. Controlled-size embryoid body formation in concave microwell arrays. Biomaterials 31, 4296-4303 (2010).

11. Kwapiszewska, K., Michalczuk, A., Rybka, M., Kwapiszewski, R. \& Brzozka, Z. A microfluidic-based platform for tumour spheroid culture, monitoring and drug screening. Lab on a chip 14, 2096-2104 (2014).

12. Desroches, B.R. et al. Functional scaffold-free 3-D cardiac microtissues: a novel model for the investigation of heart cells. American journal of physiology. Heart and circulatory physiology 302, H2031-2042 (2012). 
13. Stevens, K.R. et al. InVERT molding for scalable control of tissue microarchitecture. Nature communications 4, 1847 (2013).

14. Rivron, N.C. et al. Tissue deformation spatially modulates VEGF signaling and angiogenesis. Proceedings of the National Academy of Sciences of the United States of America 109, 6886-6891 (2012).

15. Buitinga, M. et al. Microwell scaffolds for the extrahepatic transplantation of islets of Langerhans. PloS one 8, e64772 (2013).

16. Truckenmuller, R. et al. Thermoforming of film-based biomedical microdevices. Adv Mater 23, 13111329 (2011).

17. Gottwald, E. et al. A chip-based platform for the in vitro generation of tissues in three-dimensional organization. Lab on a chip 7, 777-785 (2007).

18. Giselbrecht, S. et al. 3D tissue culture substrates produced by microthermoforming of pre-processed polymer films. Biomedical microdevices 8, 191-199 (2006).

19. Giselbrecht, S. et al. Microthermoforming as a novel technique for manufacturing scaffolds in tissue engineering (CellChips). IEE proceedings. Nanobiotechnology 151, 151-157 (2004).

20. Niles, W.D. \& Coassin, P.J. Cyclic olefin polymers: innovative materials for high-density multiwell plates. Assay and drug development technologies 6, 577-590 (2008).

21. Carpenter, A.E. et al. CellProfiler: image analysis software for identifying and quantifying cell phenotypes. Genome biology 7, R100 (2006).

22. Artus, J., Panthier, J.J. \& Hadjantonakis, A.K. A role for PDGF signaling in expansion of the extraembryonic endoderm lineage of the mouse blastocyst. Development 137, 3361-3372 (2010).

23. Plusa, B., Piliszek, A., Frankenberg, S., Artus, J. \& Hadjantonakis, A.K. Distinct sequential cell behaviours direct primitive endoderm formation in the mouse blastocyst. Development 135, 30813091 (2008).

24. Stavridis, M.P., Collins, B.J. \& Storey, K.G. Retinoic acid orchestrates fibroblast growth factor signalling to drive embryonic stem cell differentiation. Development 137, 881-890 (2010).

25. Ying, Q.L. et al. The ground state of embryonic stem cell self-renewal. Nature 453, 519-523 (2008).

26. Saiz, N., Grabarek, J.B., Sabherwal, N., Papalopulu, N. \& Plusa, B. Atypical protein kinase C couples cell sorting with primitive endoderm maturation in the mouse blastocyst. Development 140, 4311-4322 (2013).

27. Uitdehaag, J.C. et al. A guide to picking the most selective kinase inhibitor tool compounds for pharmacological validation of drug targets. British journal of pharmacology 166, 858-876 (2012).

28. Toles, J.F., Chui, D.H., Belbeck, L.W., Starr, E. \& Barker, J.E. Hemopoietic stem cells in murine embryonic yolk sac and peripheral blood. Proceedings of the National Academy of Sciences of the United States of America 86, 7456-7459 (1989).

29. Zambidis, E.T., Peault, B., Park, T.S., Bunz, F. \& Civin, C.I. Hematopoietic differentiation of human embryonic stem cells progresses through sequential hematoendothelial, primitive, and definitive stages resembling human yolk sac development. Blood 106, 860-870 (2005).

30. Dang, S.M., Kyba, M., Perlingeiro, R., Daley, G.Q. \& Zandstra, P.W. Efficiency of embryoid body formation and hematopoietic development from embryonic stem cells in different culture systems. Biotechnology and bioengineering 78, 442-453 (2002).

31. Takasato, M. et al. Directing human embryonic stem cell differentiation towards a renal lineage generates a self-organizing kidney. Nature cell biology 16, 118-126 (2014).

32. Truckenmuller, R. et al. Fabrication of cell container arrays with overlaid surface topographies. Biomedical microdevices 14, 95-107 (2012).

33. Truckenmuller, R. et al. Flexible fluidic microchips based on thermoformed and locally modified thin polymer films. Lab on a chip 8, 1570-1579 (2008).

34. Lamprecht, M.R., Sabatini, D.M. \& Carpenter, A.E. CellProfiler: free, versatile software for automated biological image analysis. BioTechniques 42, 71-75 (2007). 


\section{Supplementary information}

Below we describe the (hemispherical) geometry of the microwells and the uniformity of their distribution across the plate.

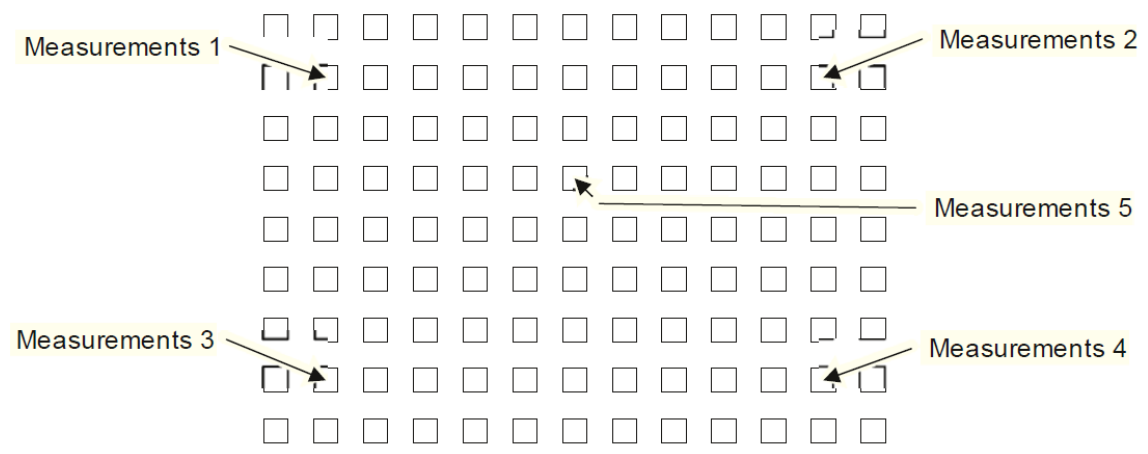

Figure S1: Optical height measurements of micro-thermoformed microwells were performed on different locations of the film.

\begin{tabular}{|l|l|l|}
\hline \multirow{2}{*}{} & Height in $\mu \mathrm{m}$ \\
\cline { 2 - 3 } & Sample 1 & Sample 2 \\
\hline Measurements 1: & 253 & 263 \\
\hline Measurements 2: & 246 & 253 \\
\hline Measurements 3: & 248 & 251 \\
\hline Measurements 4: & 275 & 271 \\
\hline Measurements 5: & 235 & 234 \\
\hline
\end{tabular}

Table S1: The microwells depth of two COP films was optically measured at five different locations and showed an uniform distribution across the film. COP material properties are: Zeonor mcs-foil 051, thickness $50 \mu \mathrm{m}$, product code: 10-0682-0000-05, supplier: microfluidic ChipShop. 


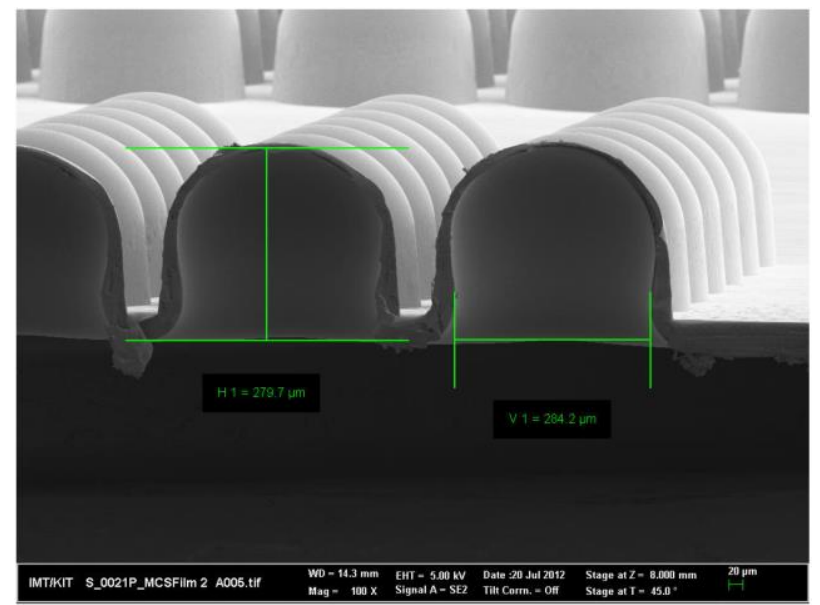

Figure S2: Validation of microwell depth. Sample 2 was cut through one row of microwells at the location of 'measurements 4 ' and the height and width was measured using SEM. The optical measurement of $271 \mu \mathrm{m}$ correlates with the SEM measurement of $279.7 \mu \mathrm{m}$.

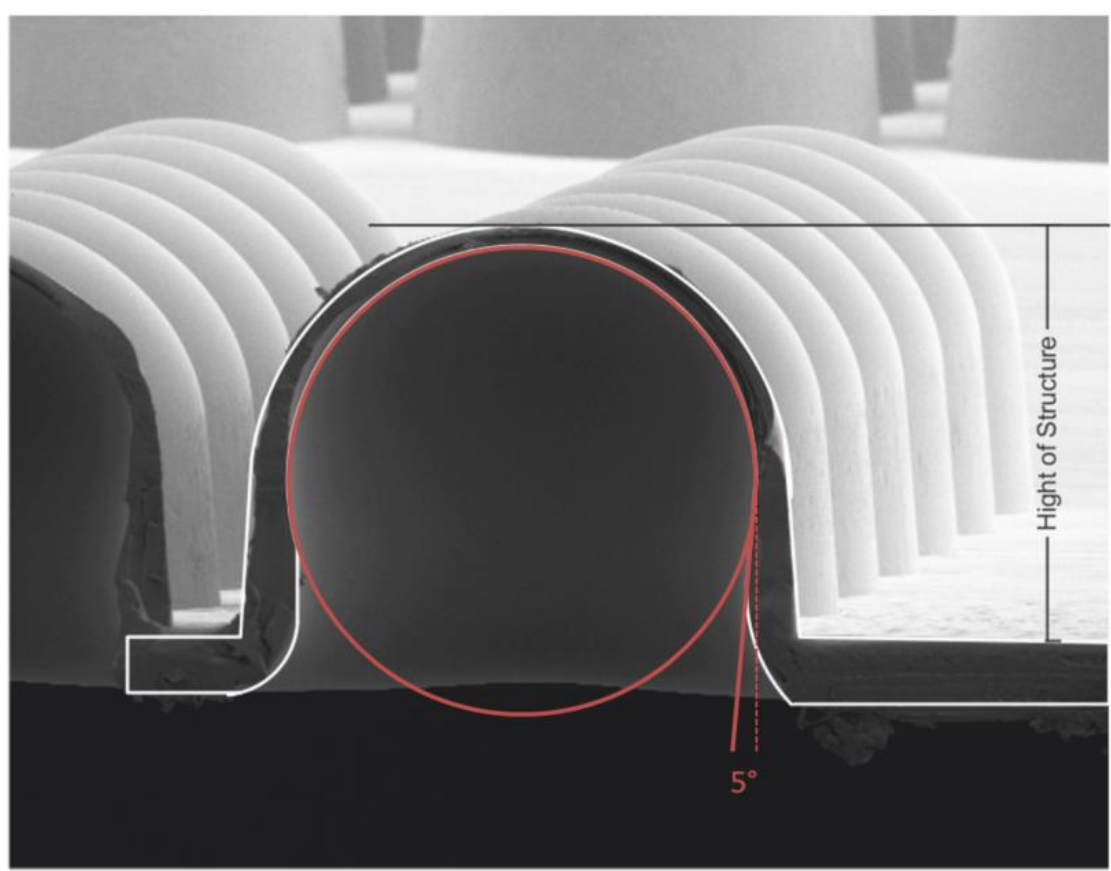

Figure S3: Microwell geometry. The microwell consists of a nearly hemispherical bottom from where the sidewalls are directed inside with a $5^{\circ}$ inclination towards the opening. The aspect ratio of depth/ diameter is almost 1.

The yield of EBs positive for PDGFRa-H2B-GFP in positive (RA), negative (2i) and neutral controls (DMSO, $\mathrm{H} 2 \mathrm{O}$ ) was compared between the thermoformed and the hydrogel plate. The results show a similar trend between the plates. 


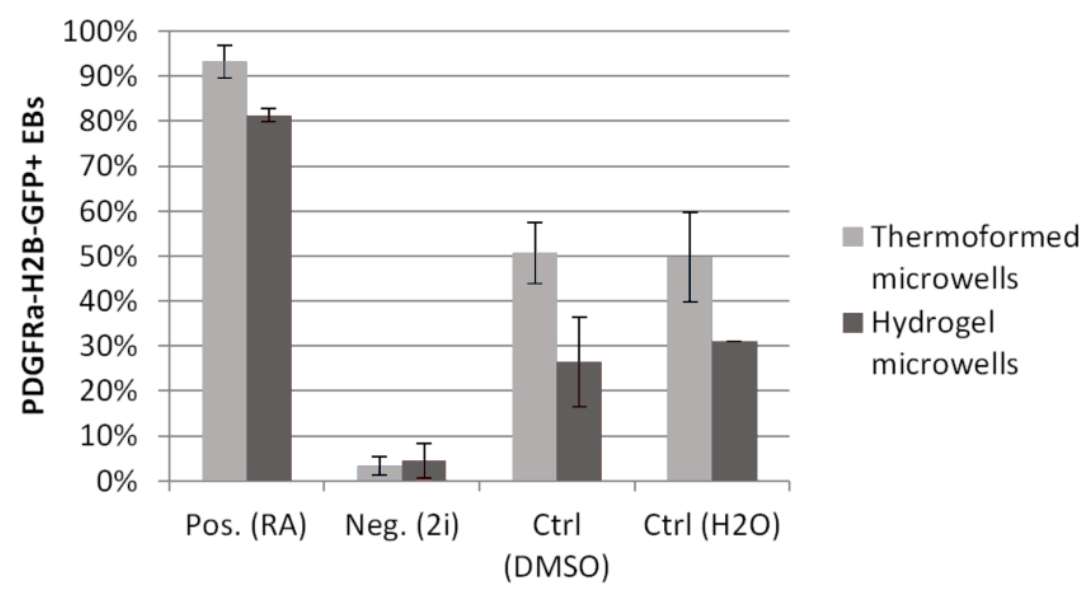

Figure S4: Yield of EBs positive for PDGFRa-H2B-GFP expression compared between plates (measured at 96 hours, $n=3$ ). 
Chapter 3 


\section{Chapter 4}

\section{Improving a Model of Preimplantation Embryonic Development using HCS}

Erik Vrij, Inês Leite, João Crispim, Javier Frias Aldeguer, Ester Caljouw, Niels Geijsen, Clemens van Blitterswijk, Roman Truckenmüller, Nicolas Rivron 
Chapter 4 


\section{Introduction}

Eukaryotic organisms exhibit diverse morphologies, yet their development covers conserved mechanisms. Our current knowledge about the molecular and genetic mechanisms that underlie the unfolding of the developmental plan of multicellular organisms has hugely benefited from biochemical and cell biological studies using model systems such as C. elegans, Drosophila, zebrafish and the mouse. Among these, the mouse model has been of foremost importance in understanding mammalian development and disease. Studies using the early mouse embryo have shed light on many developmental questions including pluripotency, lineage specification, embryonic axis formation and developmental potency. Mice are such attractive and useful models for study as they convey high biological similarity to humans. Around $75 \%$ of mouse genes have equivalents in humans ${ }^{1}$ and $99 \%$ of mouse genes have analogues in humans ${ }^{2}$. Additionally, their small scale, rapid breeding, ability to inbred, and accelerated aging compared to humans, are associated benefits that ease and speed up studies. An example of such an invaluable study is the use of mouse embryos for reproductive strategies, such as human in vitro fertilization and embryo transfer studies. Other examples are using the mouse model for cellular replacement therapies and as a biological tool to introduce and correct genetic mutations that resemble similar phenotypes of disease in humans. In this way, the mouse has become the leading model organism for experimentation in mammalian genetics and the most studied alternative for humans. In fact, due to these pioneering studies it is now possible to obtain somatic cells and reprogram them back to a pluripotent state ${ }^{3}$, which opened up a whole new field for regenerative medicine with the potential to cure degenerative diseases. Yet, many questions remain on what are the mechanisms underlying pluripotency and differentiation towards functional cells and tissues and how we can harness those mechanisms to directing these processes. Basic studies on early development of the mouse may reveal how cell lineage choice is controlled and tissues become functional. In this chapter, a methodological optimization is described that allows the formation of a cellular model that resembles early murine embryonic development to the blastocyst stage, including its stereotypical morphogenetic processes.

\section{Early embryonic development of the mouse}

Preimplantation mammalian embryogenesis encompasses the developmental course from a totipotent zygote (fertilized egg) resulting in the specification of three distinct cell lineages of the blastocyst. One consists of a group of pluripotent cells, the epiblast, that will become the embryo proper including all the organisms' adult tissues. The other two lineages, the trophectoderm and primitive endoderm (PrE), are extraembryonic and support the foetus during development ${ }^{4}$. 


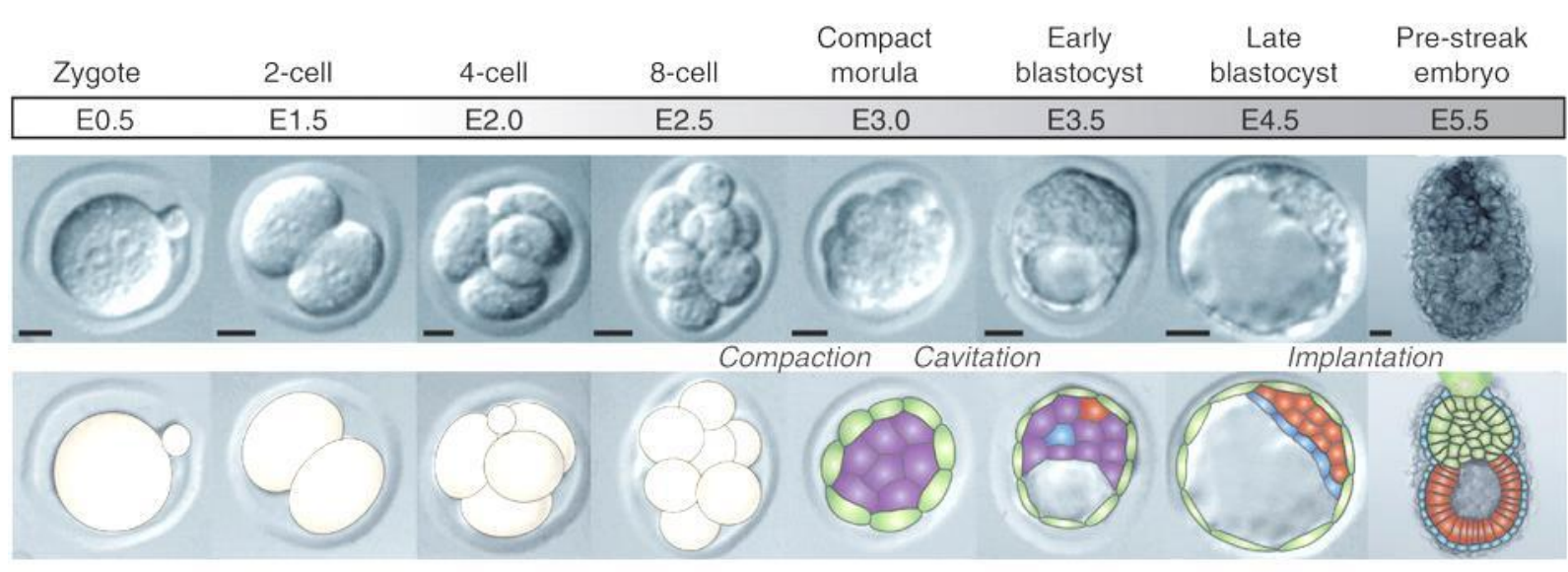

Developmental potential

Differentiation

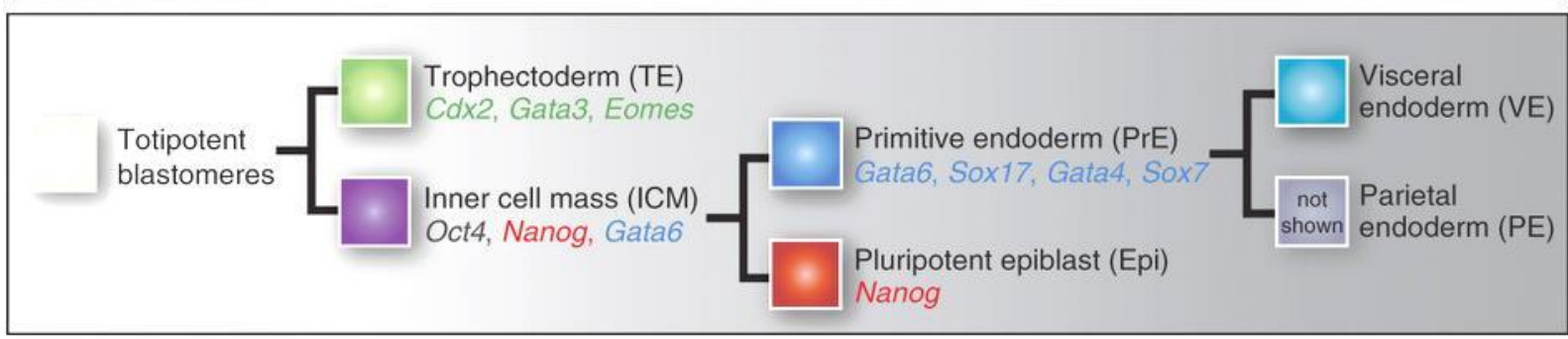

Figure 1: Stages of mouse preimplantation development. Phase-contrast microscopy images and illustrations of mouse preimplantation development, from the totipotent one-cell zygote (fertilized egg) till the post-implantation embryo at embryonic day 5.5 (E5.5). Three rounds of cell cleavage result in the 8-cell stage embryo (E2.5). Cells start to compact by the expression of tight and adherens junctions and form a solid mass of cells, the morula. Distinct outside and inside cell populations are established through (a)symmetric division. The early blastocyst is characterized by the establishment of an inner fluid filled cavity, the blastocoel. The blastocoel continues to expand in size and two distinct cell lineages can be distinguished: an outer epithelial layer, the trophectoderm (TE); and an inner cell mass (ICM). Mid and late blastocysts are then characterized with the emergence of a third distinct cell type, the primitive endoderm ( $P r E)$, which initiates in an apparent stochastic pattern amidst the ICM and segregates as an epithelial layer enclosing the ICM from the blastocoel. After implantation the PrE generates the visceral and parietal endoderm (E5.5) Scales bars are $50 \mu \mathrm{m}$. Figure adapted from ${ }^{5}$.

Development of placental mammals initiates with three successive series of cell division taking place between embryonic day $0.5(E 0,5)$ and E2,5, in which the zygote produces a cluster of 8 cells which are collectively of equal size as the zygote. These cells, also called blastomeres, are morphologically indistinguishable and retain the potential to form all cell lineages ${ }^{6,7}$. In these initial stages protein synthesis relies on maternal loaded RNA. Although molecular differences between cells are established during the rounds of cleavage from single-cell zygote to the two-, four- and eight-cell stage embryo ${ }^{8-12}$, cell fates remain plastic. Gradually, the zygotic genome is activated, Ecadherin is expressed, tight and adherens junctions start to appear and cells form a compact mass called the morula (Figure 1). Blastomeres transition from a fully totipotent state into cells with a bias for either a pluripotent or trophectoderm (TE) fate. This bias and subsequent differentiation of TE occurs prior any other body patterning event and is thus considered the first differentiated progenitor cell type that emerges during development ${ }^{13}$. Several transcription factors are known to have important functions in allocating TE and ICM fate. One key stem cell transcription factor in 
defining the identity of the trophectoderm and the ICM is Oct4 ${ }^{14}$. Before any morphological differences can be distinguished, cells in the developing embryo already show distinct Oct4 kinetics ${ }^{15}$. Another key transcription factor in maintaining the pluripotent state of the ICM (and in ES cells) is the homeoprotein Nanog ${ }^{16,17}$, which is acquired from the 8-cell stage. On the contrary, caudalrelated homeobox transcription factor $\mathrm{Cdx} 2$ is gradually acquired in precursor cells for the $\mathrm{TE}^{18}$, and represses the pluripotency factors Nanog and Oct4 ${ }^{19}$. Later during development the TE will form the extraembryonic chorio-allantoic placenta.

Around the 16- to 32-cell stage the cells in the TE form a polarized monolayer and form tight junctions, ion pumps and aquaporins that facilitate the morphogenetic process of cavitation by pumping fluid towards the basolateral side of the TE ${ }^{20}$. A blastocoelic cavity emerges around E3.5 and the embryo is now called a blastocyst, which gradually expands as more water accumulates in the cavity (Figure 1).

The ICM in the blastocyst consists of a heterogeneous population of cells that gradually diverge into the epiblast and the primitive endoderm (PrE) lineage. The activation of Gata zinc-finger transcription factors induces target genes, such as laminin and dab2, which modulate cellular adhesive function. This initiates the sorting of the two lineages and, together with selective apoptosis, leads to the formation of a PrE layer covering the pluripotent epiblast (see Figure 1) ${ }^{21}$. By the late blastocyst (E4.5), these two lineages and the TE are restricted in their future developmental fate, as was indicated by chimera studies ${ }^{22,23}$. 


\begin{tabular}{|c|c|}
\hline Term & Definition \\
\hline Totipotency & $\begin{array}{l}\text { An acquired state of cells which allows them to form all lineages of an organism. } \\
\text { In mammals only a one-cell embryo (zygote) and the first cleavage blastomeres } \\
\text { are totipotent. }\end{array}$ \\
\hline Pluripotency & An acquired state of cells \\
\hline Blastocyst & $\begin{array}{l}\text { The cystic structure that forms when a fluid-filled cavity (the blastocoel) opens up } \\
\text { in the morula. The blastocyst consists of an outer trophoblast layer surrounding } \\
\text { the blastocoel and an inner cell mass. }\end{array}$ \\
\hline Inner cell mass & $\begin{array}{l}\text { The naïve pluripotent cell mass of the blastocyst that will form the fetus and part } \\
\text { of the extra-embryonic tissues. }\end{array}$ \\
\hline Primitive endoderm & $\begin{array}{l}\text { One of the two distinct layers forming from the inner cell mass. This } \\
\text { extraembryonic cell layer later forms the extraembryonic endoderm including the } \\
\text { yolk sac. }\end{array}$ \\
\hline Epiblast & $\begin{array}{l}\text { The primed pluripotent cell mass that is formed from the inner cell mass after } \\
\text { cells from the primitive endoderm have segregated. }\end{array}$ \\
\hline Trophoblast & $\begin{array}{l}\text { Constitutes the extra-embryonic cells forming the outer polarized layer of a } \\
\text { blastocyst. It provides soluble cues and nutrients to the embryo and forms into a } \\
\text { large part of the placenta. }\end{array}$ \\
\hline Extra-embryonic tissues & $\begin{array}{l}\text { All tissues that do not contribute to the final embryo proper but facilitate } \\
\text { developing it. }\end{array}$ \\
\hline Germ layers & $\begin{array}{l}\text { The three layers of cells (ectoderm, mesoderm and definitive endoderm) } \\
\text { originating from the primed pluripotent epiblast from which all fetal and some } \\
\text { extra-embryonic tissues develop. }\end{array}$ \\
\hline Conceptus & $\begin{array}{l}\text { The embryo and its adjunct part, which includes all embryonic and } \\
\text { extraembryonic structures. }\end{array}$ \\
\hline Trophectoderm & $\begin{array}{l}\text { Encompasses the initial cells of with trophoblast identity, the polarized and } \\
\text { expanded trophoblast of the blastocyst and the derivatives of the trophoblast } \\
\text { after the blastocyst has implanted and the three germ layers have formed. }\end{array}$ \\
\hline
\end{tabular}

Box 1: Definition of terms involved in preimplantation development. Further described in ${ }^{24}$.

\section{Trophoblast differentiation: the first cell-fate decision}

Accumulating evidence shows that the apical-basal polarity of outer cells in the morula translates positional cues into molecular differences that drive differentiation of the TE (reviewed in 25-27. During morula development (8- to 16-cell and 16- to 32-cell stages) inner and outer cells progressively form by symmetric and asymmetric cell division ${ }^{28,29}$, hereby regulating the cell number ratio between the ICM and trophectoderm. The acquisition of cell polarity, including cell-cell contact asymmetries, determines cell fate choice. In fact, cell polarity has been shown to be crucial for TE specification and thus for the first cell fate decision to occur. However, whether cell position follows polarization or vice versa remains unclear. Most likely, both determinants are 
complementary in which cell position and molecular polarity reinforce the cellular identity to become either ICM or TE until these fates are restricted by the time of the 64-cell stage ${ }^{30}$.

\section{Molecular specification of the trophoblast}

Protein kinase $C(P K C)$ is a central node in controlling apical-basal polarity and thus cell fate. $A$ conserved factor is the Par3/Par6/aPKC complex, which regulates asymmetry in the distribution of membrane proteins and cytoplasmic factors ${ }^{31,32}$. The key genetic regulator for TE development, $\mathrm{Cdx2}$, is then progressively expressed in cells that inherited the polarity domain (outer cells with low cell contact) and transcriptionally promotes TE genes such as Eomes and represses pluripotency genes ${ }^{33,34}$. Yet, at the same time it is repressed in cells which inherited the basal domain (inner cells with high cell contact) ${ }^{34}$. Correspondingly, the activation of pluripotency transcription factor Sox 2 occurs in inner cells of the morula ${ }^{30}$, in conjunction with other pluripotency factors Nanog and Oct4 to coordinate ICM viability and maintenance of pluripotency. In outer TE cells, cdx2 and polarity mutually reinforce each other through the upregulation of aPKC by $\mathrm{Cd} \times 2$ and apically favoured $\mathrm{Cdx} 2$ mRNA distribution in polarized blastomeres ${ }^{10}$. It is described that $\mathrm{cdx} 2$ transcripts preferentially localize apically at the late eight-cell stage and thereby become asymmetrically inherited during cell division ${ }^{9}$. Although $\mathrm{cdx} \times 2$ is probably not required for the initial predisposition towards the TE lineage, it is essential for TE propagation and self-renewal ${ }^{8}$. Collectively, such heterogeneities in transcription factor activity between cells drive developmental lineage patterning in the early embryo.

\section{Hippo signalling}

Currently, the model that describes the delineation of TE and ICM precursors involves the regulation of Hippo signalling. The most upstream components in Hippo signalling identified so far are the adherens junctions-associated angiomotin (Amot) family and Merlin (Nf2) proteins ${ }^{35-37}$, which transduce cellular polarity into molecular signals through differential hippo signalling. In the Hippo pathway, Lats1/2 kinases phosphorylate Yap/Taz in inner cells of the early morula, which results in their degradation and inability to co-activate Tead4 target genes. On the contrary, Hippo signalling is idle in outer cells which leads to nuclear localization of Yap/Taz and, in concert with Tead4, expression of TE-specific target genes, such as Cdx2, Eomes and Gata3 (Ralston et al., 2010).

Another pathway that was described to bypass the Hippo pathway is Notch signalling. Notch directly regulates $\mathrm{Cdx} 2$ expression in concert with Tead4 and is able to drive blastomeres to the outer positions ${ }^{38}$. Hence, Notch acts in parallel to Hippo signalling.

\section{Blastocyst cavitation}

After the 32-cell stage the outer cdx2+ epithelial cells have matured to functional trophectoderm including polarized $\mathrm{Na} / \mathrm{K}$-ATPase distribution at the basolateral membrane of the TE monolayer and firm tight junctions that allow the morphogenetic process of cavitation ${ }^{39,40}$. Hence, $\mathrm{Na} / \mathrm{K}-\mathrm{ATPases}$ mediate pumping fluid across the membrane, and together with the tight junctional permeability seal of the TE, this allows the internal accumulation of fluid ${ }^{20}$. The morphogenetic process of cavitation results in the formation of a blastocyst structure, which separates the ICM and the TE layer and which is critical for further development of the embryo proper. 
Cyclic adenosine monophosphate (CAMP) is a second messenger important in many biological processes ${ }^{41}$ including a modulator of cavitation ${ }^{42}$. Its downstream actions are regulated through the exchange protein activated by CAMP (EPAC) and protein kinase A (PKA) signaling pathways. In response to CAMP stimulation aquaporins, that allow water influx, relocate from intracellular vesicles to the basolateral plasma membrane domains of the trophectoderm ${ }^{43}$. During blastocyst development cAMP levels increase slightly from $0.3 \mu \mathrm{M}$ at the 8-cell stage embryo to $1 \mu \mathrm{M}$ at the blastocyst stage. When CAMP levels are exogenously elevated in vitro culture by supplementing 1 $\mathrm{mM}$ of cAMP analog the rate of blastocyst formation significantly increases ${ }^{44}$, which was shown to be cAMP/PKA signalling mediated.

Blastocyst formation is also regulated by mobilization of intracellular $\mathrm{Ca}^{2+}$ stores through the IP3 signalling pathway ${ }^{45}$. Interestingly, exposure of $0.1 \%$ ethanol (and not of related alcohols such as isopropanol) stimulates blastocyst cavitation and expansion through a transient increase in intracellular calcium levels ${ }^{46}$.

\section{Primitive endoderm formation: the second cell-fate decision}

The second cell-fate decision involves cells from the ICM committing to either the primitive endoderm (PrE) or epiblast (EPI) lineage. Initially, the ICM is a heterogeneous cell population with cells co-expressing markers of PrE and EPI ${ }^{47}$. As development progresses, and cavitation occurs, Nanog expression is repressed in some cells in conjunction with an upregulation of Gata6 expression ${ }^{48}$, while in other cells Nanog levels are maintained ${ }^{49}$. Gradually, cells within the ICM become mutually exclusive for Nanog and Gata6 in a seemingly random salt \& pepper distribution. Gata6+ cells commit to the PrE lineage, including expression of markers such as Gata4, serpinh1 and PDGFRalfa ${ }^{21}$. Cells biased towards the PrE lineage start to emerge at least as early as the 16-cell stage ${ }^{50}$. The initial signals leading to the formation of these two lineages remains unclear. However, the progressive formation of PrE and allocation of cells with a PrE identity involve several mechanisms such as cell sorting and selective apoptosis ${ }^{21}$. Movement of PrE precursor cells towards the ICM surface is associated with the formation of cell protrusions ${ }^{51}$, also seen in with scanning electron microscopy. By the late blastocyst (E4.5) stage the PrE has formed a morphologically distinct epithelial monolayer on the surface of the EPI ${ }^{52}$. By then, all three lineages are restricted in their future developmental fate as was indicated by chimera studies ${ }^{22,23}$. The extra-embryonic tissues, including the TE and PrE and derivatives, are involved in patterning the early embryo and providing a foetal-maternal connection which is required to sustain post-implantation embryonic development. For both rounds of cell fate decisions (TE versus ICM, and PE versus EPI) accounts that a first wave of signalling biases cell fate, which is then reinforced by lineage-specific transcription factors. 
E3.5

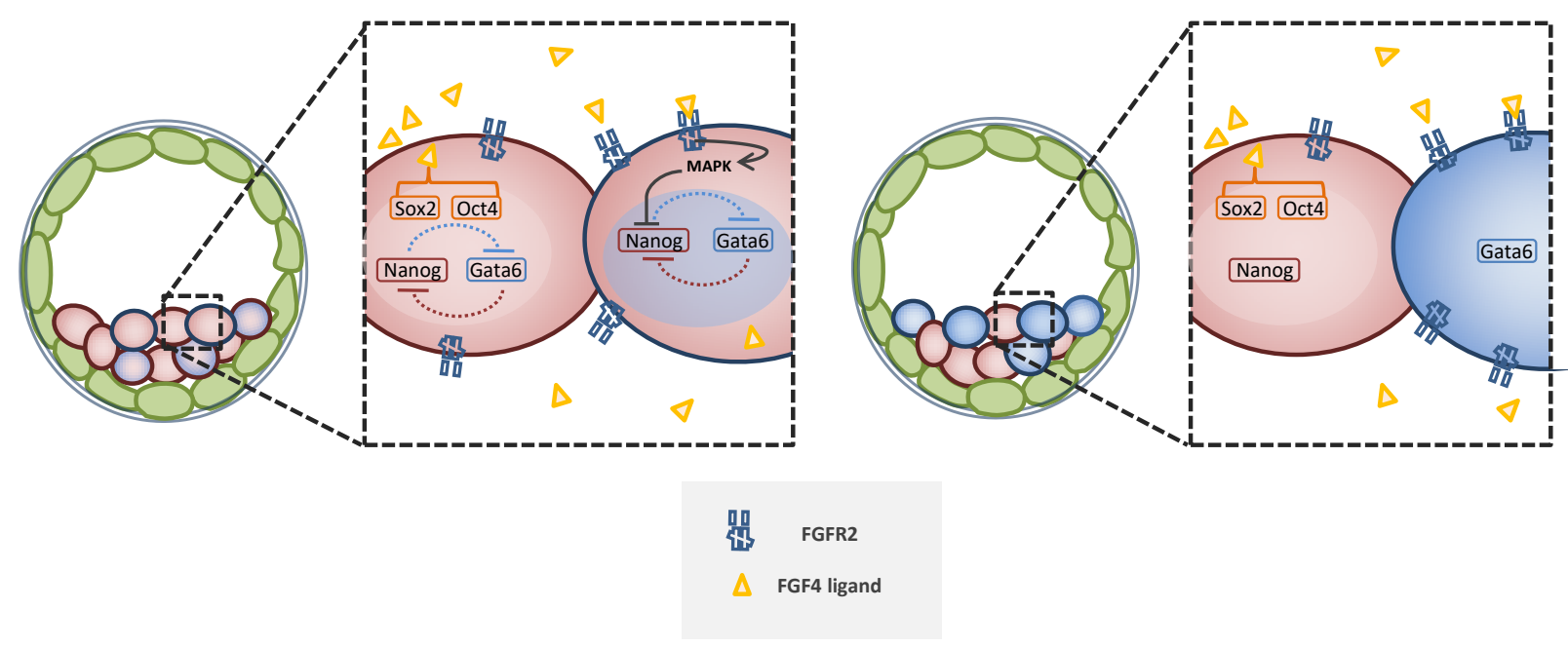

Figure 2: PrE identity reinforcement by FGF signaling. (A) At the onset of cavitation, cells in the ICM express an overlap in FGF4 and FGFR2 transcripts. pluripotent factors promote the expression and secretion of FGF4 which binds to FGFR2, transduces via MAPK signalling and increases Gata6 expression. Gata6 represses Nanog and enhances expression of PrE genes. (B) Around the 64-cell stage (E3.75), this FGF signalling circuit has reinforced itself and mutually exclusive lineages of PrE and pluripotent epiblast have established.

\section{Signalling pathways involved in PrE specification and development}

Diverse molecular mechanisms orchestrate the morphogenetic processes that culminate in the peri-implantation blastocyst. The key signaling cascade involved in PrE specification is FGF signaling.

\section{FGF signaling}

FGF signaling is a recognized component involved in PrE formation. The ligand FGF4 is secreted by the pluripotent epiblast and binds to the FGF receptor 2 on Gata6-expressing PrE progenitor cells, where it reinforces the PrE identity ${ }^{53,54}$. FGF signaling is not required for the initial co-expression of FGF4 and FGFR2 but is involved in the followed mutual exclusion of PrE and epiblast-associated transcription factors that positively feedback on this circuit which culminates into the establishment of a salt-and-pepper distribution of lineage-biased cells in the ICM (Figure 2) ${ }^{55}$. This mutual repression circuit is reinforced by other FGF signaling components such as FGFR3 and FGF3 ${ }^{54}$ and is suggested to be a general network architecture to integrate the activity of signal transduction pathways and transcriptional regulators ${ }^{56}$. The binding of FGF4 to the receptor causes the receptor tyrosine kinase (RTK) domain to initiate the RTK-Grb2-Sos-Ras-MEK-Erk signaling cascades, which play a crucial role in lineage specification ${ }^{57}$. This is emphasized by peri-implantation lethality in mice carrying mutations in the FGF4 gene ${ }^{58}$. This FGF/MAPK signaling pathway produces a delicate balance between cells of EPI and PrE specification in the ICM, as an excess of FGF4 ligand drives all ICM cells into the PrE lineage ${ }^{53}$, whereas a mutation of FGF4 restricts the ICM from differentiating into PrE and maintains Nanog-high levels ${ }^{55}$. A similar phenotype is observed when blocking the FGF/MAPK signaling downstream such as in embryos with a mutant form of the adaptor protein Grb2 ${ }^{52}$ or when using chemical MAPK inhibitors (PD032 ${ }^{59}$ ). Interestingly, the mechanism that initiates the heterogeneity in FGF4/FGFR2 expression is still unknown and could be stochastic ${ }^{60}$. 
However, ICM cells internalized at different times during development show a differential response to FGF signaling which influences lineage segregation ${ }^{61}$.

\section{PKC signaling}

Atypical protein kinase $\mathrm{C}(\mathrm{aPKC})$ expression is enriched within PrE precursors in the ICM and is involved in cell sorting and PrE maturation. After cell sorting, when PrE cells reach the blastocyst cavity, aPKC adopts a polarized localization in these cells ${ }^{62}$.

\section{Jak/Stat signaling}

It was recently shown that downstream of LIF signaling the Jak/Stat cascade promotes the expansion of ICM cells primed for PrE within the blastocyst ${ }^{63}$. Exogenous addition of LIF increased the number of Gata6+ cells, whereas inhibition of Jak/Stat signaling reduced both Nanog+ epiblast cells and Gata6+ cells. This indicates a role for LIF-producing cells in modulating the size of the PrE layer.

\section{post-implantation development}

Right after implantation, by E5.0, cells from the PrE start to differentiate, migrate and segregate into two subpopulations of extra-embryonic endoderm. One of those populations consists of parietal endoderm (PE) cells which scatter over the inner surface of TE giant cells and secrete basement membrane proteins to form the Reichert's membrane ${ }^{64}$. The early functioning transient parietal yolk sac is derived from the thick Reichert's membrane in conjunction with PE cells and trophoblastic giant cells ${ }^{65}$. The other PrE derived subpopulation is the visceral endoderm which differentiates from the PrE cells in contact with the epiblast ${ }^{66}$. By E8.0 the closely associated extra-embryonic mesoderm and VE form the visceral (definitive) yolk sac, in which the VE plays an inductive role in the formation of blood islands amongst other functions ${ }^{67,68}$.

\section{Developmental plasticity}

Dissociated blastomeres from both the inner or the outer compartments of 16-cell stage embryos are able to form blastocysts after re-aggregation and develop into normal mice ${ }^{69}$. In these blastomeres-aggregates $\mathrm{Cdx} 2$ and Oct4 expression was re-established accordingly, which implies at least some blastomeres were still totipotent. Moreover, cells from 32-cell stage embryos that were randomly re-aggregated repositioned their cells accordingly and gave rise to mice. However, the selecting for inner or outer cells from the 32-cell stage embryo did not yield embryos capable of full development. This implies that by the time of a 32-cell stage embryo the cells are yet committed to their respective states. The major aberrant phenotype of isolated blastomeres-derived embryos are trophoblastic vesicles devoid of an inner cell mass ${ }^{69-71}$. In another study cell-cell interaction and cell position was eliminated by continuously separating the blastomeres after each cell division ${ }^{72}$. This resulted in mixed expression of ICM- and TE specific genes in single blastomeres, but preferentially of TE fate. Also, when blastomeres were re-aggregated they formed partly developmentally-capable blastocysts and blastocysts with a smaller ICM. Although maternal factors are important for mammalian development ${ }^{73}$, no protein factors have been identified that crucially affect cell fate in the embryo. This strongly indicates that maternal determinants do not govern preimplantation 
development. This in contrast to other organisms, such as flies and frogs, in which developmental information about head and tail location is encoded within the fertilized egg ${ }^{74,75}$.

Clearly, multiple determinants govern the progressive cell fate allocation of and segregation. These include the differential activity of transcriptional regulators, inter-cellular cues through cellcell contact and soluble factors, and polarity, both inherited during (a)symmetric cell division and acquired through positional state. Redundancy in these signals, at least during a certain time window, provides the embryo with flexibility to adjust when perturbed and safeguards the correct specification of the primary cell lineages ${ }^{25,26,76}$. For example, blastocysts that are artificially twinned result in smaller embryos but progress development synchronously with their un-split sibling controls ${ }^{77}$. How the molecular timing of developmental milestones is achieved still remains elusive.

\section{In vitro expansion of embryonic stem cell lines}

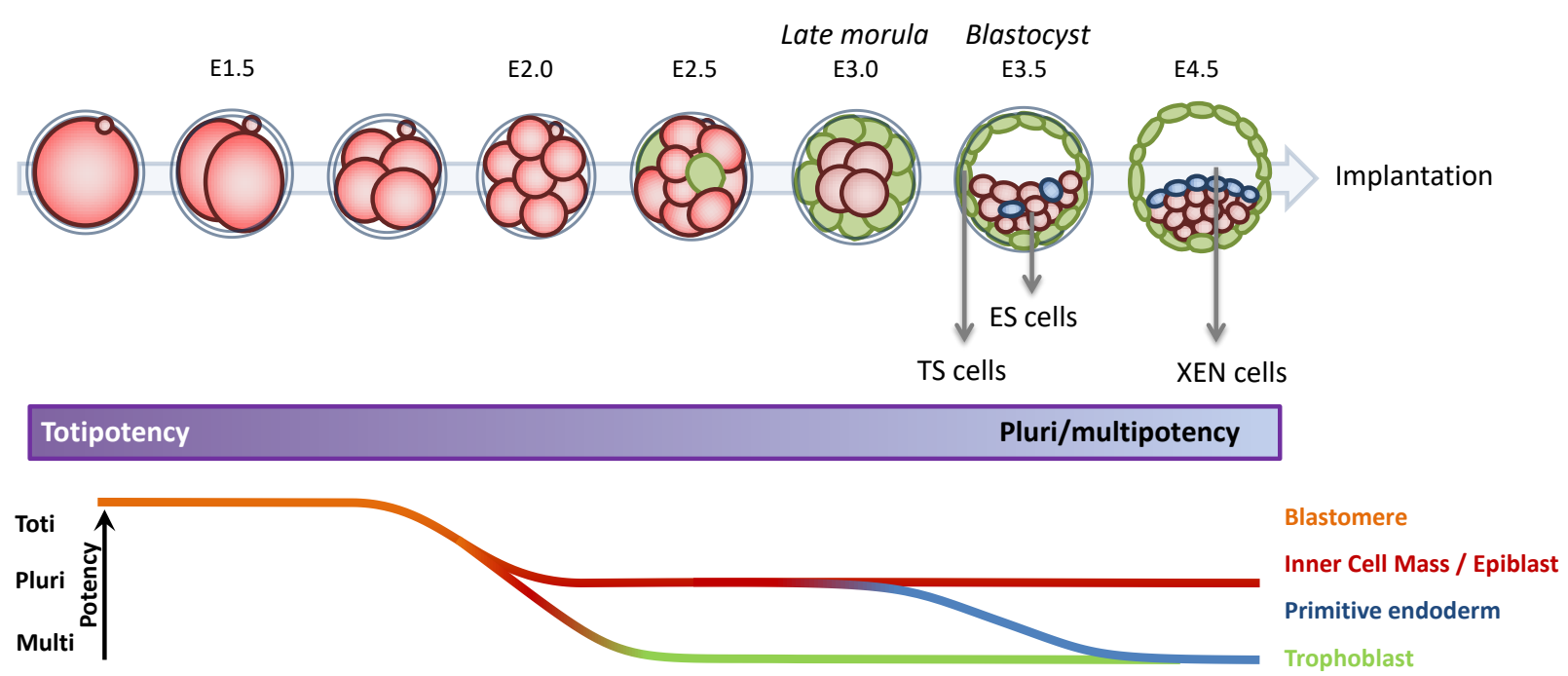

Figure 3: The totipotent cell mass gradually acquires lineage bias. The three distinct lineages that are specified during preimplantation development can be derived from the embryo and expanded in vitro.

\section{In vitro pluripotency: naïve versus primed}

Totipotent stem cells are so far impossible to expand in vitro. Yet, pluripotent mouse embryonic stem (mES) cells can be derived from the late inner cell mass or epiblast of the blastocyst and can be propagated in vitro culture indefinitely by a process of symmetrical self-renewal (Figure 3). In contrast to adult stem cells, which also possess the ability to self-renewal, mES cells have a pluripotent identity which permits them to differentiate in any adult tissue. Self-renewal in mES cells is characterized by rapid proliferation and high telomerase activity, which allows these cells to proliferate indefinitely without accumulating genetic anomalies and maintain their pluripotent identity. A unique feature of mES cells is their unusually short G1 phase and lack of regulation at the G1-to-S phase transition (reviewed by ${ }^{78}$.

Mouse ES cells can be expanded on feeder cells in serum-containing medium supplemented with leukemia inhibitory factor (LIF) ${ }^{79}$. In these conditions a state of 'primed' pluripotency is established 
which includes heterogeneous expression of pluripotency markers and presence of developmentally progressed cells ${ }^{80}$. Alternatively, a culture regime termed $2 i$ culture was found that enables the propagation of mES cells in a 'naïve' ground state of pluripotency ${ }^{81}$, characterized by more homogenous expression of pluripotency markers such as Nanog, Stella, Pecam1, klf4 and Rex1 ${ }^{82}$. Herein, two small molecules are supplemented in the culture media, PD0325901 and CHIR99021, that inhibit the MEK/ERK pathway and activate the canonical Wnt pathway (inhibition of GSK3 $\beta$ ) respectively. This $2 \mathrm{i}$ culture of $\mathrm{mES}$ cells is characterized by more homogenous expression of pluripotency markers such as Nanog, Stella, Pecam1, klf4 and Rex $1^{82}$. Specifically Rex 1 is a validated marker correlating closely with naïve pluripotency ${ }^{83}$. Time lapse studies have shown that a drastically higher portion of serum/LIF expanded mES cells is eliminated after blastocyst injection compared to $2 \mathrm{i} /$ LIF expanded ES cells ${ }^{84}$.

\section{Epiblast stem cells}

Another heterogeneous but pluripotent cell population, named epiblast stem cells (EpiSC), can be expanded in vitro, and represents the epiblast of the early post-implantation stage embryo ${ }^{85}$. Omitting Wnt signaling in ES cells triggers differentiation to EpiSCs ${ }^{86}$, which can form teratomas but are restricted in chimera contribution.

\section{Trophoblast stem cells}

Multipotent stem cells can be derived from the extraembryonic lineages of a blastocyst. Hence, cells from the trophoblast can be expanded in vitro as trophoblast stem (TS) cells (Figure 3), which can give rise to all the trophectoderm derivatives in vivo ${ }^{87,88}$. To promote their self-renewal and multipotency in serum containing medium the activation of FGF and Activin/Nodal signaling is required ${ }^{89}$. Activin is either secreted by mouse embryonic feeder (mEF) cells or giant trophoblast cells or supplied exogenously. Recently, several serum-free culture protocols have been developed, which include a matrigel-based substrate and the addition of TGF- $\beta$ in case of so-called Tx-medium ${ }^{90}$, and in other reports the ROCK inhibitor Y27632 either supplemented with Activin A and a Wnt inhibitor ${ }^{91}$ or supplemented with a pan-retinoic acid receptor inverse agonist and a fibronectin/laminin substrate ${ }^{92}$.

\section{XEN cells}

Extraembryonic endoderm cells can be derived from the blastocyst (Figure 3), expanded in vitro culture exhibiting self-renewal and contribute to the extraembryonic endoderm cell types in chimeras ${ }^{5,93}$. These cells represent the primitive endoderm of the blastocyst stage embryo. Several protocols have also described the differentiation of ES cells into XEN-like cells either using growth factors retinoic acid and Activin $A^{94}$ or the conversion of ES into XEN cells using forced overexpression of Gata6 ${ }^{48}$ or Sox $17{ }^{95}$. Moreover, aggregating ES cells into embryoid bodies (EBs) results in an outer layer of XEN cells ${ }^{96,97}$. 


\section{The blastoid: an in vitro model of preimplantation embryonic development of the mouse}

\section{Embryoid bodies as state-of-the-art models for embryonic development}

To recapitulate the spatial organization of embryonic development in vitro researchers have developed multicellular models based on embryonic stem cells. These models have enabled researchers to perform detailed studies on the molecular mechanisms of embryonic patterning. Thus far, aggregates of embryonic stem cells termed embryoid bodies (EBs) have been the primary model to recapitulate and study particular aspects of embryonic development, such as symmetry breaking and axial organization ${ }^{98}$ or the identification of transduction pathways involved in establishing polarity ${ }^{99}$. To faithfully recapitulate embryonic development, however, the extraembryonic trophectoderm should be included. Here, we developed a stem cell-based model of murine preimplantation development consisting of a pluripotent embryonic compartment surrounded by a trophoblast-derived blastocoel. Unarguably, as discussed, the early mouse embryo has greatly facilitated studies of mammalian embryonic development. However, embryos are far from amenable to the powerful tools of high-throughput screening and phenotype-based highcontent screening. Additionally, ethical concerns of animal welfare are actively debated in society.

\section{The Blastoid: an artificial blastocyst}

Morphogenetic processes during development, including cell fate allocation, segregation and cavitation, are governed by a compounding set of molecular and positional cues. Collectively, these mechanisms provide an inherent robustness for the embryo which allows it to adjust when perturbed. This flexibility is demonstrated by the embryo's ability to self-reorganize, for example when blastomeres or ICM cells are removed, morulae are disintegrated or blastocysts are split. Moreover, isolated individual ICM cells from the early blastocyst (32- to 64-cell stage) retain the ability to form blastocyst-like structures ${ }^{100}$. This indicates that these cells, similar to blastomeres, can polarize, respond to positional cues and form TE epithelium ${ }^{71}$. Interestingly however, this plasticity disappears at the point when the blastocyst is fully expanded and ready for implantation ${ }^{101,102}$. The cell types that can be derived from the blastocyst, i.e. ES, TS and XEN cells, each merely recapitulate developmental features restricted to their respective niche.

Here, we use a model termed the blastoid; a multicellular system formed by a combination of ES cells and TS cells to recapitulate preimplantation development more accurately than existing models. Blastoids comprise both the embryonic and extraembryonic compartments and display similar morphology and cell allocation to blastocyst-stage embryos. They are formed by positioning the ES and TS cells into a structure resembling the late morula assisted by cellular self-assembly. Subsequently, processes of self-organization combined with exposure to the proper chemical cues drives differentiation and morphogenesis. 


\section{Results}

\section{Formation of the blastoid}

In the blastoid, TS and ES cells are associated to form a structure that recapitulates a congregation of trophectoderm and inner cell mass, respectively. Upon modulation of signal transduction pathways active blastocysts TS cells cavitate around an aggregate of ES cells, resulting in a structure that is morphologically analogous to a blastocyst.

Multiple ES cell lines have been used for the formation of blastoids, although with varying effectiveness. Generally, ES cells were first expanded for several rounds and up to copious numbers using naïve culture conditions consisting of ES medium supplemented with LIF and GSK3 and Mek/erk inhibitors (2i culture), either on a feeder layer of mouse embryonic fibroblast (mEF) or directly on gelatin-coated dishes (Figure 4A). Correspondingly, TS cells were expanded on mEF in TS medium ${ }^{103}$ freshly supplemented with FGF4 and heparin (Figure 4B).

A

Embryonic Stem Cells

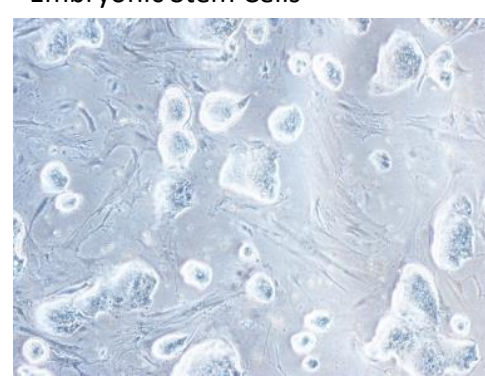

B

ES medium
LIF
CHIR
PD32 $\} \begin{aligned} & 2 i \\ & \text { condition }\end{aligned}$

\section{Trophoblast Stem Cells}

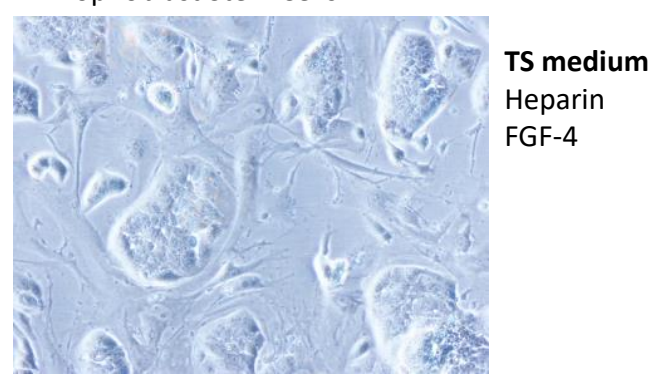

Figure 4 - Expansion of embryonic and trophoblast stem cells. A) Colonies of embryonic stem cells cultured in naïve conditions on a feeder layer of mEF. B) Colonies of trophoblast stem cells expanding in TS medium on a feeder layer of mEF. Images taken using phase contrast microscopy.

To allow the parallelized culture of hundreds of blastoids within the same condition, we utilized the microwell screening array platform. This platform, as described in Chapter 2, comprises of a standard 96-well plate with in every well an array of 430 cylindrical hydrogel microwells. Each microwell with a diameter of $200 \mu \mathrm{m}$ allows the spontaneous aggregation of cells that settle within and thus permits the potential formation of a blastoid structure.

Late morula stage embryos consist of approximately 22 cells in total, which proliferate to approximately 45 cells when cavitation starts around E3.25 ${ }^{62}$, with a ratio of TE:ICM cells of 1.67 For that reason, a total number of around 22 cells were seeded to allow the formation of an initial ES cell body engulfed by TS cells. This is done by first seeding an average target number of 6 ES cells that aggregate into an embryoid body within 24 hours of culture (Figure 5, step 1). Then, around 17 TS cells are seeded in on top to engulf the EB by forming a tight monolayer around it (Figure 5A, step 2). This step is complemented with the addition of specific small molecule signal transduction 
modulators: 8Br-cAMP to activate CAMP/PKA signaling, CHiR to activate canonical Wnt signaling and y27632 to inhibit Rock signaling (Figure 5, step 3).

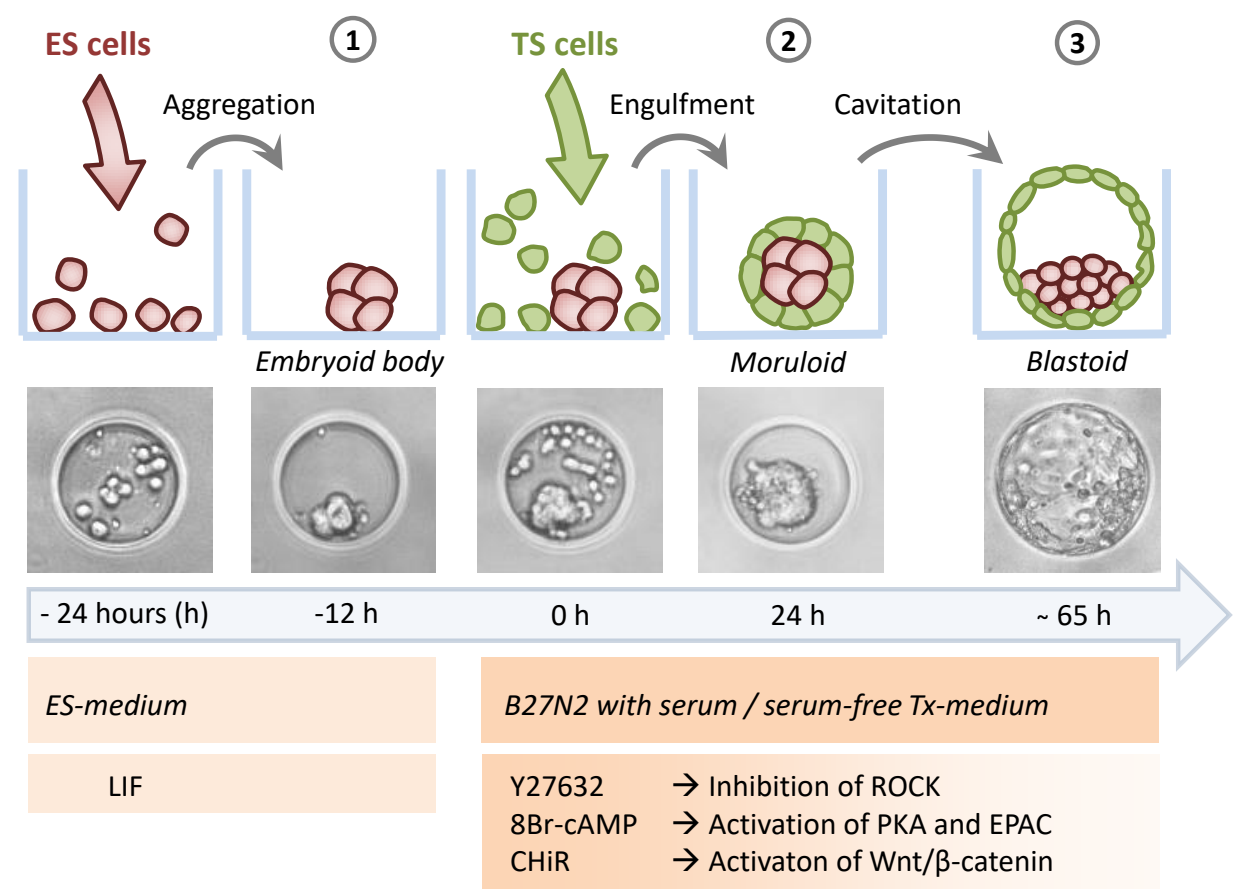

Figure 5: Cellular aggregation, organization and formation of blastoids depicted by schematics and phase contrast light microscopy images. A) Approximately 7 ESC cells per microwell aggregate into an embryoid body of about $25-50 \mu \mathrm{m}$ in diameter and are cultured for 24 hours in ES medium before medium is substituted with B27N2 blastoid culture medium together with the addition of approximately 17 TS cells per microwell. TS cells engulf the ESC aggregate within 24 hours and initiate cavitation around 48 hours of culture and continue pumping liquid inward. Around 65 hours of culture in blastoid medium a blastocyst-like structure has formed including a fluid filled cavity with an inner cell mass.

\section{Engulfment of ES cell aggregates by TS cells}

Inhibition of Rock signaling has been shown to affect the hippo signaling in zygotes and in early pre-compaction stage embryos, thereby enhancing the pluripotent ICM lineage and leading to reduced Cdx2 expression in TE precursors ${ }^{104,105}$.. However, when y27632 was added beyond the 8cell stage development was not critically affected. Inhibition of Rock signaling by adding y 27632 during blastoid culture helps positioning the TS cells surrounding the ES cell compartment. The prototype of a successfully engulfed structure morphologically resembles a late stage morula and has an inner core of pluripotent ES cells with a monolayer of TS cells surrounding it (Figure 6A, step 2), which are termed moruloids. Using fluorescently labeled V6.5 mouse ES cell line containing a fusion protein of $\mathrm{H} 2 \mathrm{~B}$ and RFP which are constitutively expressed (red colored fluorescence) and live stained TS cells using CellTracker Green CMFDA dye (green colored fluorescence), such moruloids can be straightforwardly recognized using epifluorescence microscopy. High-content imaging and high-content automated analysis were exploited to obtain quantified data effectively describing the morphological features of these moruloids. This was done using a method which calculates the 
overlap in projection area between a synthetized ring around the inner embryonic core and the TS cell layer (Figure 6A, step 2). Clustering the Y27632 (Rho/ROCK inhibitor) treated group versus the control group on circularity (vertical axis) and engulfment efficiency (horizontal axis) showed that Y27632 treated moruloids were more circular and had a higher engulfment efficiency than moruloids from the control group (Figure 5A, step 3).

\section{ROCK inhibition and modulating TS cell number}

Rho-associated protein kinase (ROCK) is an intracellular serine-threonine kinase (family of protein kinase A, among others) and downstream effector of Rho. The ROCK pathway is a key regulator of actin organization (stress fibre formation) including coordinating effects on cellular morphology, contractility, adhesion and migration ${ }^{106}$. Among other processes, ROCK is also involved in apoptosis through its activation by the pro-apoptotic protease caspase 3 . Consistently, selective inhibition of ROCK using the small chemical Y27632 in human and mouse embryonic stem cell culture permits increased survival of cells after dissociation ${ }^{107}$. Y27632 is often used at a concentration around $10 \mu \mathrm{M}^{108}$ and, importantly, does not show long-term cytotoxic effects nor does it negatively affect ESC pluripotency.

For optimizing the blastoid culture conditions we performed an exponential titration range for Y27632, which resulted in the highest engulfment efficiency for working concentrations between 5 and $20 \mu \mathrm{M}$ (Figure 6B). Similarly, a TS cell number titration range yielded an optimal number of 17 TS cells (Figure 6C). And indeed, on fluorescence images it can be clearly seen that moruloids efficiently engulfed with 17 TS cells using $20 \mu \mathrm{M}$ Y27632 also had a higher circularity (Figure 6D). Finally, as epifluorescence images are only a 2D reflection, we performed confocal microscopy to obtain data about the positional information of cells in the 3D structure. This confirmed that moruloids identified as efficiently engulfed actually consist of an ES cell-based compartment enclosed by TS cells (Figure 6E). 
A
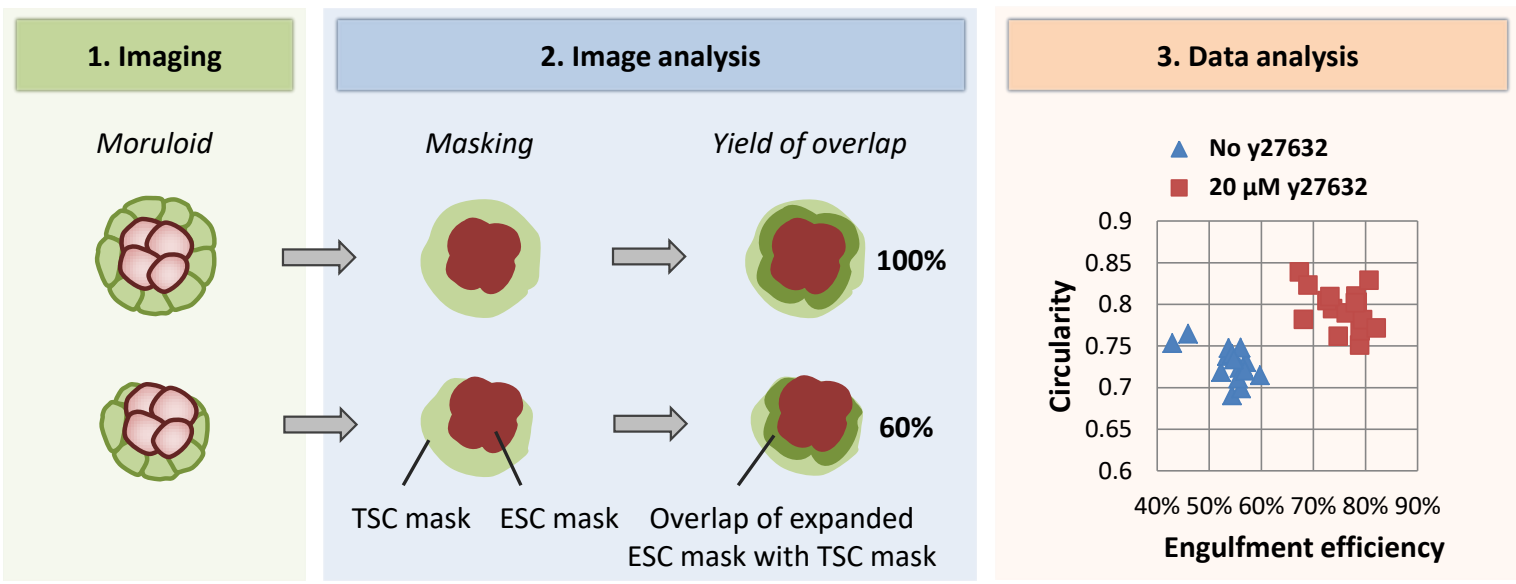

B

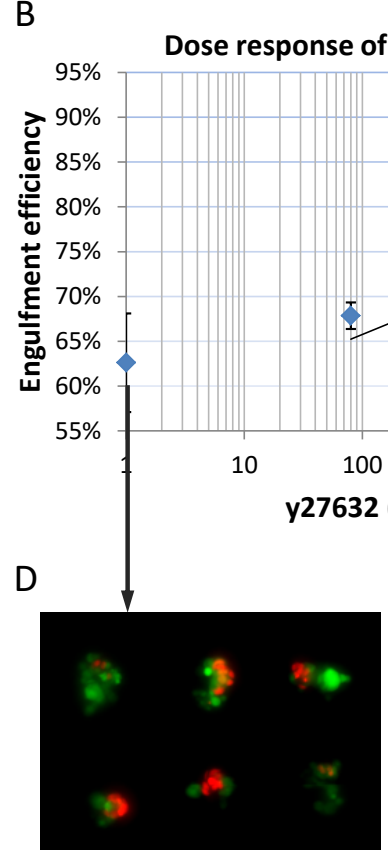

$0 \mu \mathrm{M}$ of Y27632 (ROCKi)
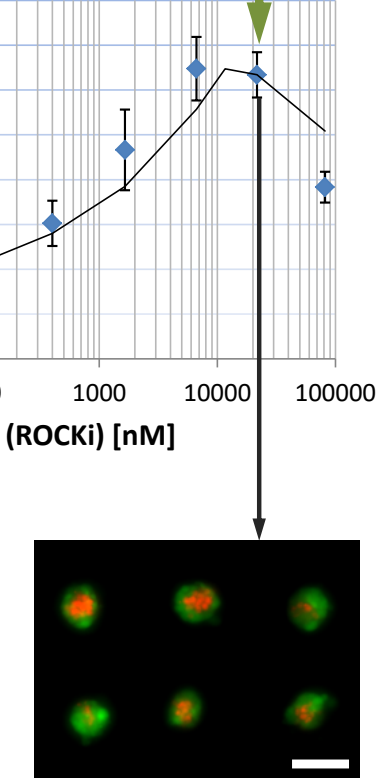

$20 \mu \mathrm{M}$

C

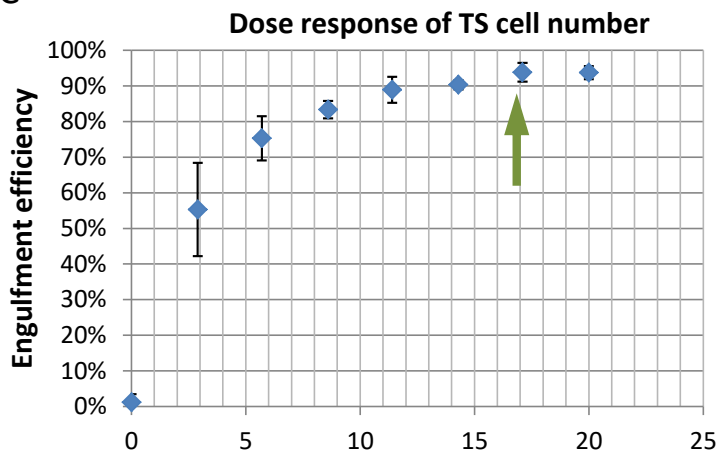

Number of TSCs per microwell [1]

$y$

$y$

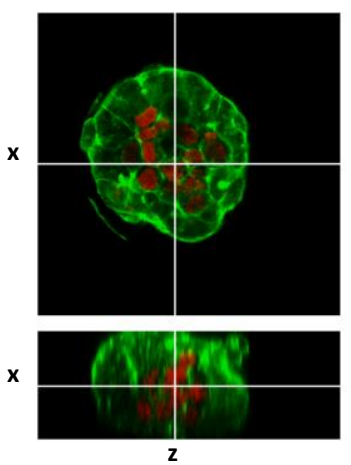

Figure 6: Engulfment efficiency of moruloids. (A) Schematic of high-content image analysis. The degree of engulfment is measured as the percentage of overlap of fluorescently labelled TS cells (light green) with an artificially introduced layer (dark green) around the ES cell aggregates (red). Circularity versus engulfment efficiency of ESC bodies with TSC shows two clusters. (B) Engulfment efficiency of ES cell bodies by TS cells in response to an increasing concentration of Rho/ROCK inhibitor Y27632 (24 hours after seeding TS cells, $n=6$ wells, median of $\sim 250$ moruloids per well. The groups including 1650 $\mathrm{nM}$ and higher are statistically different from $0 \mathrm{nM}$ with a confidence level of $\mathrm{p}<0.001$ ). The arrow depicts the concentration of y27632 that was chosen to be used in further experiments. (C) Engulfment efficiency of ES cell bodies by TS cells in response to increasing number of TSCs (24 hours after seeding TS cells, $n=6$ wells, median of $\sim 250$ moruloids per well. The group of 17 (arrow) cells is statistically different from the groups of 0,3 and 6 cells with a confidence level of $p<0.001)$. (D) Representative fluorescence images depicting differences in engulfment efficiency of ES cell bodies (H2B-RFP nuclear reporter depicted in red) and TS cells (stained by CellTracker green) 24 hours after seeding TS cells. (E) Orthogonal images made by confocal microscopy showing an ES cell body engulfed by TS cells (H2B-RFP nuclear reporter depicted in red, F-actin depicted in green). 


\section{Cavitation}

\section{High-content imaging and analysis}

Using high-content imaging analysis the number and morphometries of blastoids can be identified and quantified. First, a number of at least 200 microwells per well are captured using montaged epifluorescence imaging as an end-point measurement (96 hours). Multicellular structured comprised of ES cells with a H2B-RFP reporter and TS cells, counterstained for F-actin using Phalloidin-488. The moruloids that were adequately engulfed were amenable to form a blastoid. As such, every microwell contained either a structure that had developed into a blastoid or a non-cavitated cluster of ES and TS cells (Figure 6A, step 1). After image acquisition, Cell Profiler was used to discriminate the blastoids from non-cavitated structures based on a radial intensity profile of F-actin (Figure 6A, step 2). Then, the yield of blastoids was determined and of all structures the projection area of the total structure and of the ICM, including morphometric features such as circularity (Figure 6A, step 3).

\section{Activation of cAMP/PKA signaling}

cAMP levels can be pharmaceutically modulated by compounds such as Forskolin (activates the upstream enzyme adenylyl cyclase), 8Br-cAMP (a CAMP analogues) and H-89 (a cAMP/PKA inhibitor). As previously described, cAMP signaling during embryonic development mainly affects the CAMP/PKA pathway. The stress related components of the CAMP-activated pathway EPAC1 and EPAC2 are dispensable for normal development ${ }^{109}$. Moreover, selective activation of EPAC1 using the cAMP analogue 8-CPT-2Me-CAMP-AM (007AM) did not lead to increased cavitation (data not shown). Conversely, 8Br-cAMP increased the yield of blastoids slightly, but the total blastoid size remained constant (Figure SI 1). Interestingly, omitting the addition of $8 \mathrm{Br}$-cAMP still resulted in a fair yield of blastoids (32\%, Figure SI 1 ). However, cAMP/PKA might either be endogenously activated or by factors in the serum such as GPCR ligands that are known to signal via CAMP ${ }^{110}$. Moreover, inhibition of CAMP/PKA by H-89 led to a reduced yield and smaller blastoids

More importantly, we found that CAMP(/PKA) activation resulted in a larger ICM, maintaining ES cell viability. To study the dose effect, $8 \mathrm{Br}$-cAMP was administered at $0 \mathrm{~h}$ and $48 \mathrm{~h}$ in concentrations ranging from 0 to $5 \mathrm{mM}$ and exposed to blastoids for prolonged culture time (96 hours) before fixation. It was found that a concentration of $1 \mathrm{mM}$ led to the largest ICM in blastoids (Figure 6B). When $200 \mu \mathrm{M}$ or $1 \mathrm{mM}$ of $8 \mathrm{Br}$-cAMP was supplemented, the PA of the embryonic compartment within blastoids showed a significant increase of $116 \%$ and $130 \%$, respectively, compared to no $8 \mathrm{Br}$ CAMP ( $p<0.001)$. The addition of 8Br-cAMP not only improved ICM maintenance in blastoid but also in non-cavitating structures (Figure $6 \mathrm{C}$ ). Compared to $1 \mathrm{mM}$ of $8 \mathrm{Br}$-cAMP, the addition of $5 \mathrm{mM}$ slightly compromises cystic expansion (measured as PA) and reduced the yield of cystic structure formation by 12 and $9 \%$, respectively (Figure SI 1). Yet, the latter values do not differ in a statistically significant manner. Altogether, the results suggest that the optimal concentration of 8Br-cAMP to induce blastocyst-like structures can be found around $1 \mathrm{mM}$. 
A

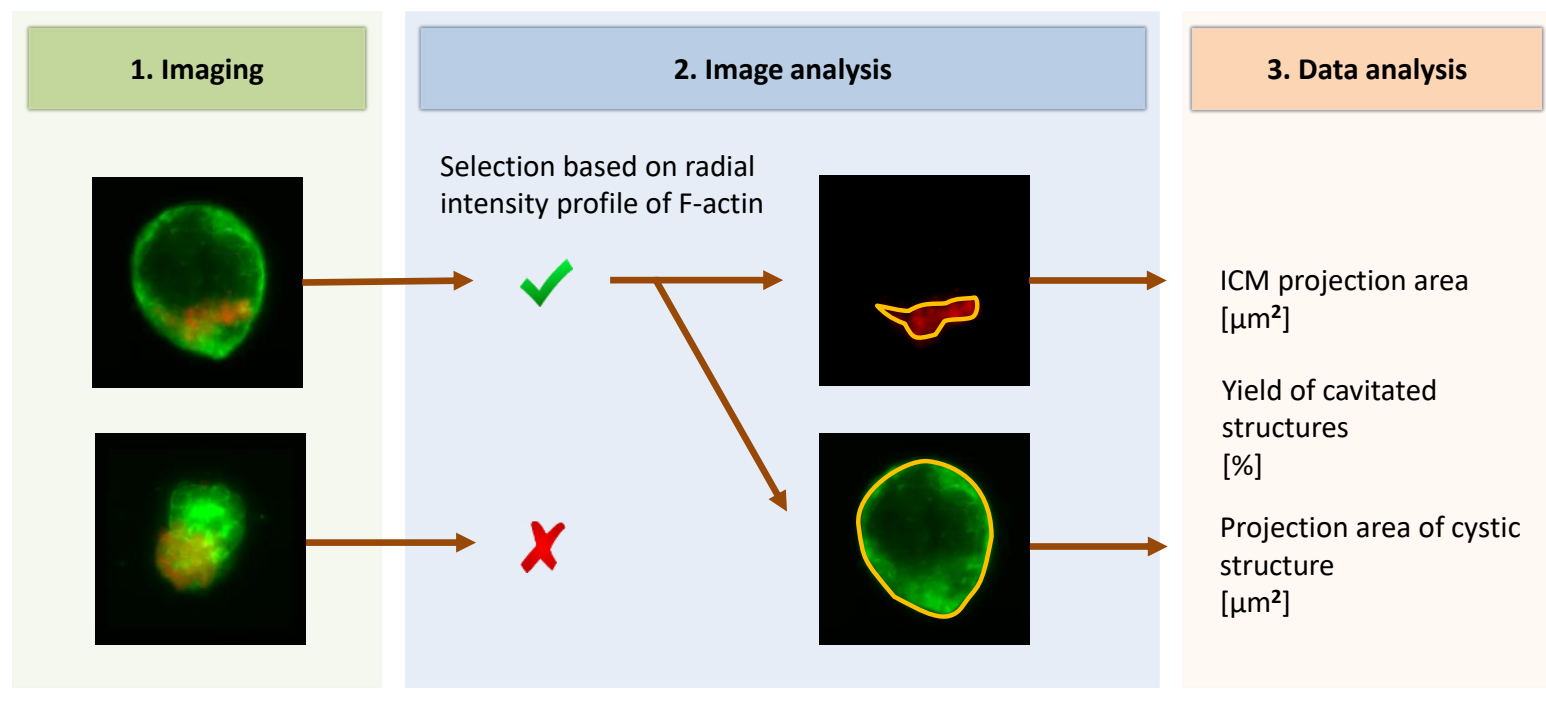

в

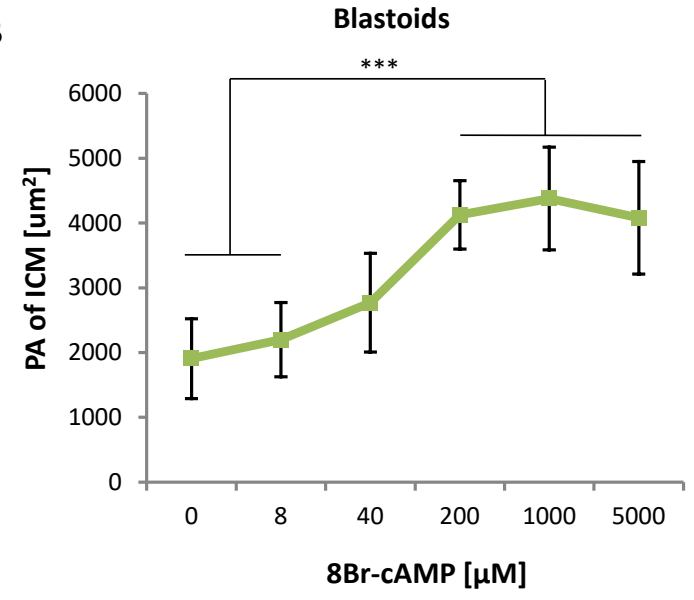

C

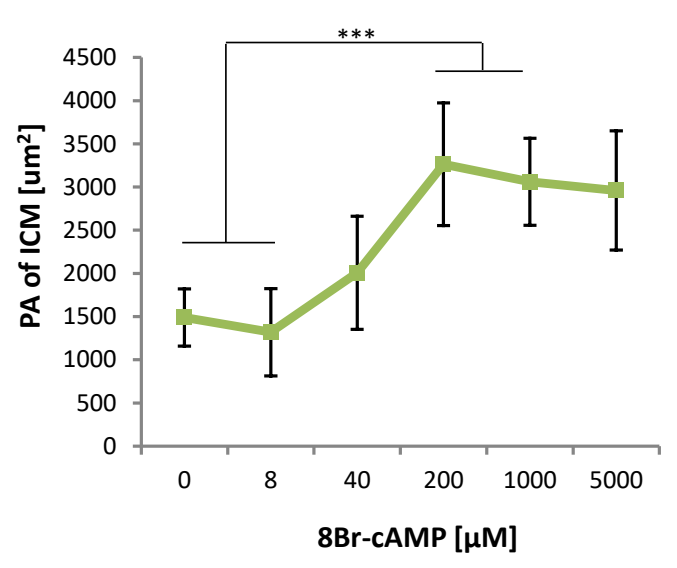

Figure 6: CAMP/PKA signaling maintains the ICM of blastoids. (A) Schematic of image processing, including: 1) high-content imaging, where up to 430 structures are imaged per well; 2 ) image analysis, where a selection of corresponding structures are identified, linked and outlined; 3) data quantification and analysis. (B) Projection area of the embryonic compartment (ICM) for cavitated structures (blastoids) and (C) non-cavitated structures at 96 hours of culture. A tipping point for maintaining the ICM appears to be positioned between 200 and $1000 \mu \mathrm{M}$ of $8 \mathrm{Br}$-cAMP. $\mathrm{n}=6, * * *$ denotes $p<0.001$, ANOVA with Bonferroni post-test. 


\section{Mutual regulation of the embryonic compartments}

\section{The embryonic compartment regulates trophectoderm development in vitro}

Aggregates of embryonic stem cells are able to form cystic structures that resemble the visceral yolk sac endoderm ${ }^{111,112}$. As EBs do not comprise a trophectoderm layer, they are not molecularly or mechanically guided in their development by this extraembryonic tissue and therefore lack resemblance to blastocyst development. Here, on the contrary, the formation of a morula-like structure (named moruloid) including a polarized trophectoderm monolayer surrounding the EBs enables the formation of a blastocoel through cavitation. It is known that the inner cell mass and the trophectoderm interact ${ }^{113}$. The blastoid system permits modulating the number of ES cells in the embryonic compartment to study the effect on trophectoderm development.

By dosing the average number of seeded ES cells per microwell from 0 to 14 data was obtained on the yield of cavitated structures (yield of formed blastoids and cystic EBs), the projection area of the embryonic compartment (EBs and blastoids) as a proxy of ICM size, and the projection area of the blastoids and EBs as a proxy of their total size. The average number of ES cells, that make up the 'ICM', modulated the yield of cystic structures (blastoids) that formed (Figure 7B), probably via paracrine signaling, juxtacrine signaling or both. An optimal yield of blastoids was obtained with an average number of 6 ES cells per blastoid (91\% higher compared to no ICM, $p<0.001$ ). The projection area of the 'ICM' showed, as expected, a positive correlation with the average ES cell number (Figure 7B). However, notably, the increase in ICM size stabilized with higher ES cell number (Figure $7 C$ ). This can be an artefact resulting from the method of measuring the ICM size, which is a 2D representation of a 3D compartment. Alternatively, it could be an internal regulation of pluripotent stem cells within the ICM by modulating proliferation or apoptosis for instance, as is seen in native development ${ }^{114}$.

Additionally, the total projection area (PA) of blastoids increased with the presence of an embryonic compartment and showed a dependence on the number of ES cells (Figure 7C). Correspondingly, it has been shown that prior to implantation a minimum of four pluripotent cells should be present to prevent developmental arrest ${ }^{77}$. The largest blastoids formed when 6 ES cells were seeded. The PA of blastoids seeded with 6 ES cells was $70 \%$ larger than when no ICM was present $(p<0.001)$. Notably, both in terms of blastoid yield and total size, the trophectoderm development reached an optimum when an average of 6 ES cells was seeded to form moruloids (Figure 7D). Interestingly, this number corresponds with the number of pluripotent ICM-precursors that are observed during embryonic preimplantation development at the E3.0 stage of morula compaction ${ }^{115}$. These results indicate that trophectoderm development is tightly regulated by the embryonic compartment.

Upon seeding cells onto the microwell array, cells from the bulk volume stochastically settle down within the microwells. As of this, the number of seeded cells within the microwells followed a normal distribution and the average value corresponded with the targeted cell seeding number 
(Figure 7E). The optimal target seeding number of 6 ES cells resulted in an average of 6.88 ES cells per microwell.

Overall, concluding from these results, the size of the embryonic compartment in blastoids, which resembles the inner cell mass (ICM) of blastocysts, regulates the development of the trophectoderm.

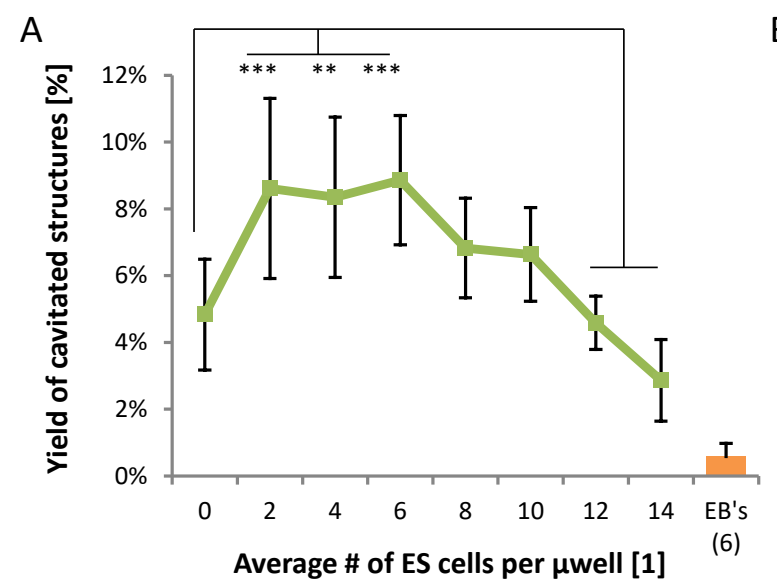

C

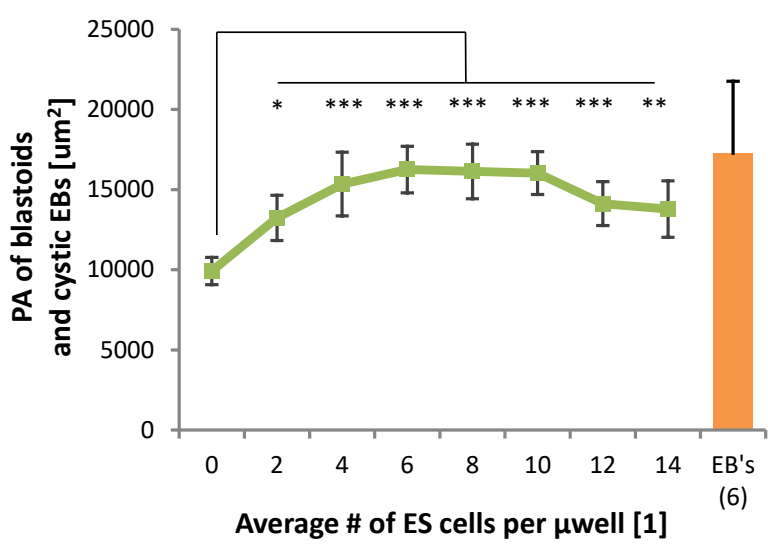

B

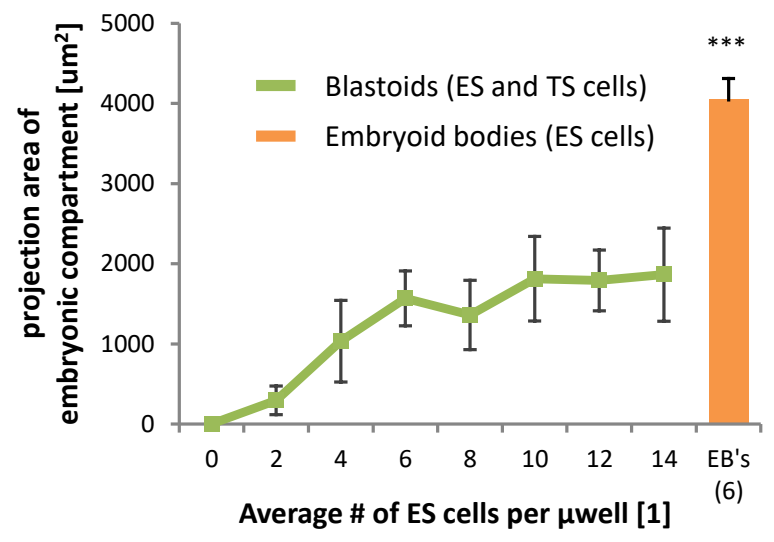

D
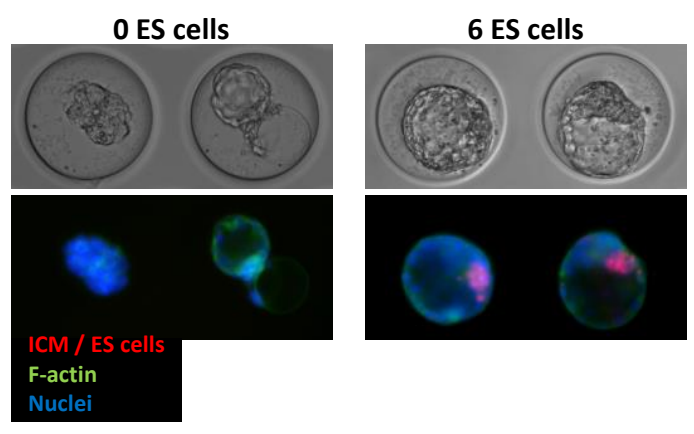

$E$

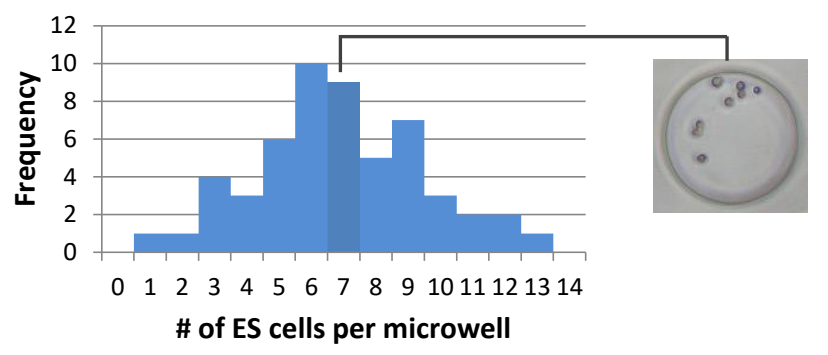

Seeding target: $\quad 6$

Average: $\quad 6.88$

Median: $\quad 7$

Figure 7: Modulating the embryonic portion affects trophectoderm development and morphogenesis. (A) Yield of cavitated structures after 69 hours of culture: blastoids (green) and EBs (orange). The average number of ES cells within the embryonic compartment affects the yield of blastoids with a maximum at 6 ES cells. (B) The projection area of the embryonic compartment increases with the average number of ES cells per structure. EBs from 6 ES cells show a projection area more than $2 x$ larger than the corresponding embryonic compartment in blastoids. (C) The projection area of cystic structures (blastoid and cystic EBs). The number of ES cells in the embryonic compartment regulates the size of the blastoid cavity, with the largest size at 6 ES cells. (D) 
Representative phase contrast and fluorescence images of a non-cavitated structure and an empty blastoid (left), and two blastoids including an ICM (right). (E) Distribution of the number of ES cells per microwell when a targeted number of 6 ES cells was seeded. ${ }^{* * *}$ denotes $p<0.001, n=9$ wells, ANOVA with Tukey's multiple comparison test.

\section{The trophectoderm regulates proliferation of the embryonic compartment}

In Figure 7B it was observed that EBs grow larger over time than the embryonic compartment within blastoids. Now, the projection area (as a proxy for size) of the embryonic compartment was measured for EBs, blastoids and non-engulfed (non-cavitated) structures (Figure 8A). While the initial cell number was similar (a target seeding number of 6 ES cells per microwell), after 72 hours of culture the EBs were substantially larger than the ICM of blastoids $\left(4073 \mathrm{um}^{2}\right.$ for blastoids versus 1864 um $^{2}$ for EBs), and only slightly bigger than the embryonic compartment of non-engulfed structures (Figure 8B). The results indicate that a blastocyst-like morphology including the trophectoderm slows down the proliferation of ES cells. This is underlined by the finding that nonengulfed bodies of ES cells (non-blastoids) were less severely restricted in their growth than blastoids (Figure 8A). Also, this excludes the possibility of nutrient depletion as the primary cause for restriction of the embryonic compartment, as TS cells were added in both conditions (blastoids and non-engulfed structures). Instead, physical restriction such as the stiff F-actin rich TS cells that engulf the ICM or the higher osmotic pressure within the blastocoel could play a role. However, paracrine or juxtacrine signals may also be involved as the embryonic compartment of non-engulfed structures is yet smaller than EBs.

A

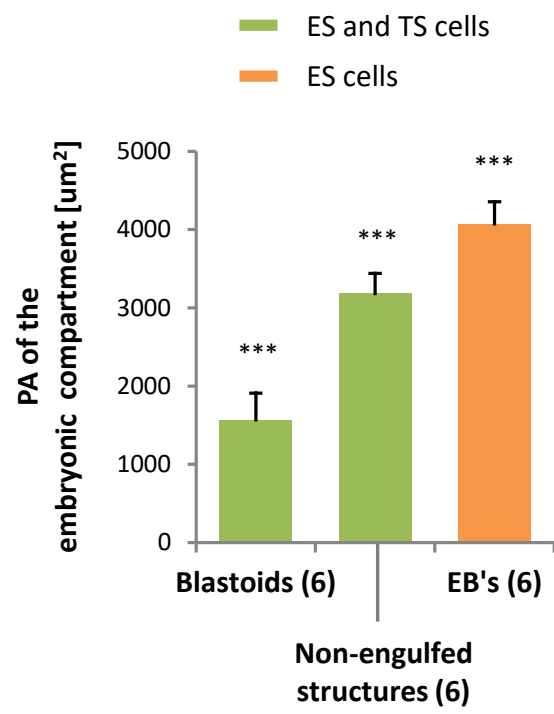

B

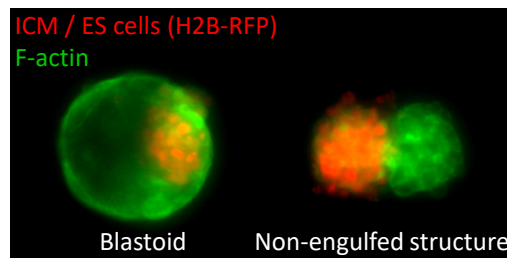

Figure 8: The trophectoderm restricts the growth of the embryonic compartment. (A) The embryonic compartment of structures non-engulfed by TS cells grow bigger than those of blastoids. EBs grow to the largest size. (B) Illustrative images of a blastoid and a non-engulfed structure. ${ }^{* * *}$ denotes $p<$ 0.001 between all conditions, $\mathrm{n}=9$ wells, ANOVA with Bonferroni's multiple comparison test. 


\section{Primitive endoderm formation}

\section{Downregulation of Nanog}

During blastocyst formation cells within the ICM gradually reinforce a mutually exclusive identity of either pluripotent epiblast or primitive endoderm. This coincides with overall lower Nanog expression in the embryonic compartment as primitive endoderm precursors start to repress Nanog. Here, ES cells with a knock-in allele for a TdTomato reporter for Nanog were used to form blastoids and cultured until appropriate time points after being live stained using Hoechst and imaged. Figure 9A displays the average fluorescence intensity of the Nanog-TdTomato reporter within the ICM of blastoids, which shows a decline from $72 \mathrm{~h}$ to $96 \mathrm{~h}$ (artificial units, $\mathrm{p}<0.01$ ). This time window corresponds with the formation of PrE in blastocysts ${ }^{26}$.

\section{Lineage segregation within the embryonic compartment}

Using an ES cell line comprising a fluorescent histone $\mathrm{H} 2 \mathrm{~B}$ reporter under the control of the PrE marker PDGFR $\alpha$ (PDGFR $\alpha-\mathrm{H}_{2} \mathrm{~B}-\mathrm{GFP}{ }^{21,116}$ ) blastoids can be formed that show a green fluorescent signal in cells that drive into the PrE lineage. Using a high-content screening approach 430 blastoids per well of a 96-wellplate can be analyzed for the presence of this reporter (Figure 9B). Blastoids were counterstained for F-actin using Phalloidin-594 to discrimate between blastoids and noncavitated structures. Occasionally, a blastoid with PDGFR $\alpha-H 2 B-G F P+$ cells lined-up on top of embryonic compartment was observed, which was then further analyzed using confocal microscopy (Figure 9C). Often, however, PDGFRa-H2B-GFP+ cells were found in a unsystematic distributed manner within the ICM. Approximately $3 \%$ of all structures had one or more PDGFR $\alpha-H 2 B-G F P+$ cells in the ICM. (Figure 9D). 
A

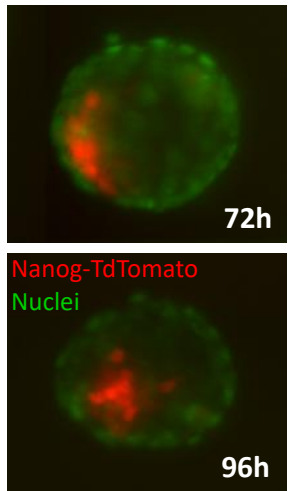

C

Confocal microscopy images
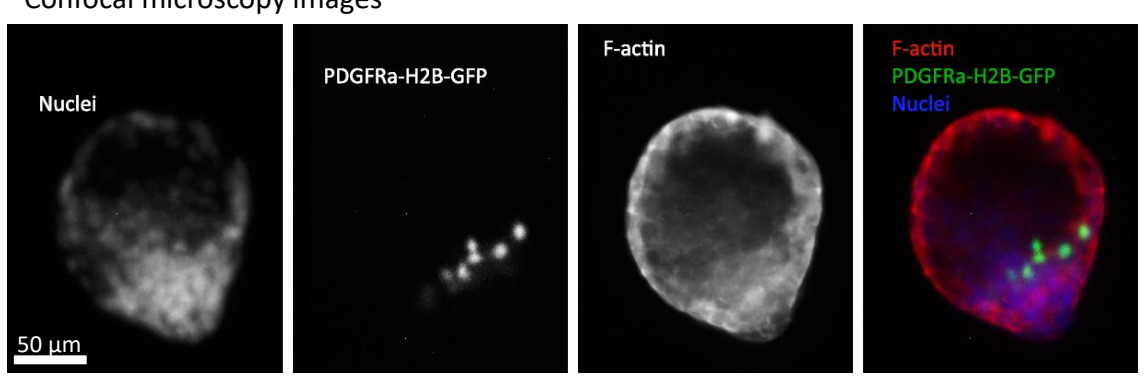

D
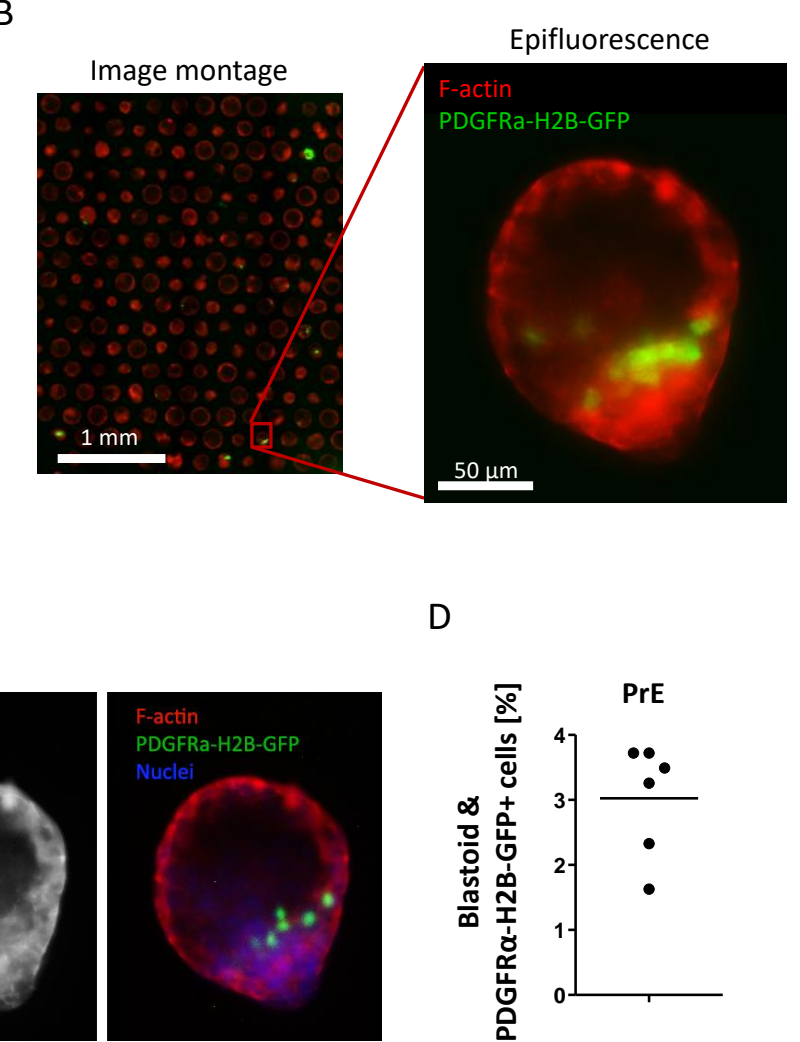

Figure 9: Primitive endoderm formation within the blastoid. (A) Fluorescence intensity of NanogTdTomato reporter in the ICM of blastoids decreases during development from $72 \mathrm{~h}$ to $96 \mathrm{~h} .{ }^{* *}$ denotes a $p<0.01, n=5$, T-test. (B) Epifluorescence montage image showing blastoid morphology in red ( $F$ actin staining with Phalloidin-594) and primitive endoderm in green (PDGFR $\alpha$-H2B-GFP reporter). (C) Confocal images of a blastoid with a lined-up layer of primitive endoderm marked by PDGFRa-H2BGFP+ cells. (D) Yield over the total amount of structures of blastoids including PDGFR $\alpha-H 2 B-G F P+$ cells in the ICM. 


\section{Optimizing blastoid formation}

\section{Cascading yield loss}

Beyond cavitation, the next hallmark of preimplantation development is the formation of primitive endoderm, which emerges as Gata6+ cells, and later PDGFR $\alpha+$ among other markers, within the ICM in a seemingly stochastic fashion after which they segregate from the Nanog+ cells and form an epithelial sheet on top of the epiblast. Unfortunately, blastoid development is subject to cascaded yield loss, which means that every successive step progressively results in a lower yield (Figure 10). Proper statistics requires a minimal number of biological replicates per condition, which necessitate the optimization of every single step of blastoid formation.
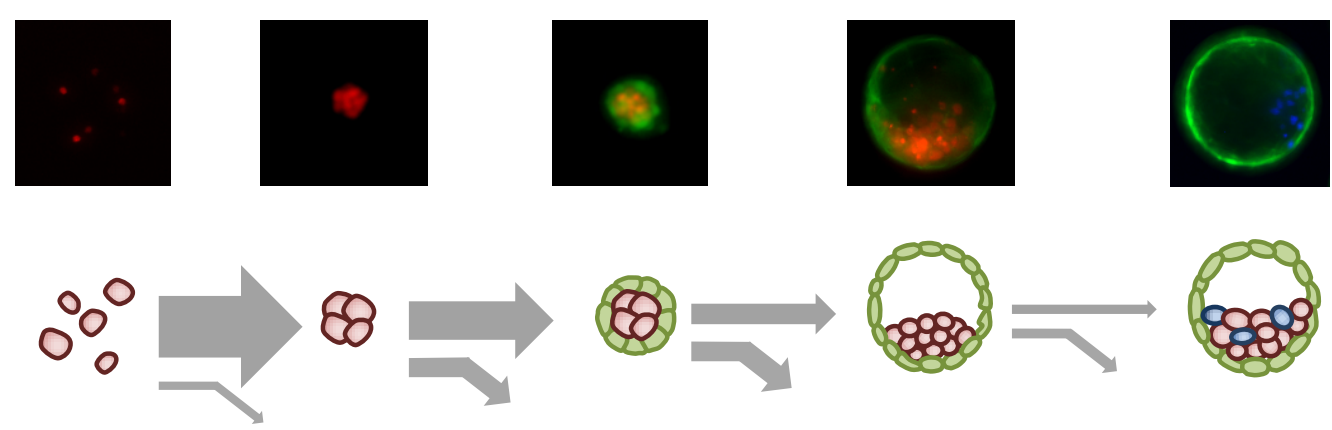

ES cells

Trophectoderm PDGFR $\alpha+$ cells

Figure 10: Cascading yield loss in blastoid culture. With every step in development the accumulated loss in yield increases.

\section{Histone deacetylase inhibitor SAHA}

Often, moruloids that show a proper engulfment of TS cells do not cavitate. Here, the small molecule suberanilohydroxamic acid (SAHA, tradename Vorinostat) that belongs to the class of histone deacetylase inhibitors ( $\mathrm{HDACi}$ ), was found to promote the yield of cavitation. HDAC inhibitors have a wide spectrum of epigenetic activities and have been used for inducing pluripotency and differentiation ${ }^{117}$. SAHA was previously found to reduce apoptosis in blastocysts, facilitate the generation of nuclear transfer-derived embryonic stem cell lines and improve the development of cloned mice to full-term without obvious abnormalities ${ }^{118}$. Also, it was shown to upregulate STAT3 signaling in multiple cell lines, which controls ES cell self-renewal and maintains pluripotency ${ }^{119}$.

\section{SAHA boosts the yield of cavitated structures}

When administered at the start of blastoid culture, SAHA showed an improvement in cavitation yield (Figure 11A) with increasing concentrations up to $200 \mathrm{nM}$. SAHA was found to act by synergizing with CHiR. Hence, adding 200nM SAHA without CHiR increased the cavitation yield negligibly (from $4 \%$ to $6 \%$ ). In contrast, supplementing cultures with $200 \mathrm{nM}$ SAHA and $6 \mu \mathrm{M} \mathrm{CHiR}$ significantly boosted the yield of blastoid formation; from $15 \%$ to almost $26 \%$ (Figure $11 \mathrm{~A}$ and $\mathrm{B}$ ). 
Then, dosing the concentration of $\mathrm{CHiR}$ with and without SAHA showed a CHiR dose-dependent increase in yield by SAHA (Figure 11C). Ramping up CHiR concentrations to $10 \mu \mathrm{M}$ or higher resulted in yields of more than 50\%. However, particularly in combination with $200 \mathrm{nM}$ SAHA, this led to a reduction of ICM size in blastoids (Figure 11D). This suggests a toxic effect on ES cells for CHiR concentrations higher than $6 \mu \mathrm{M}$.

A

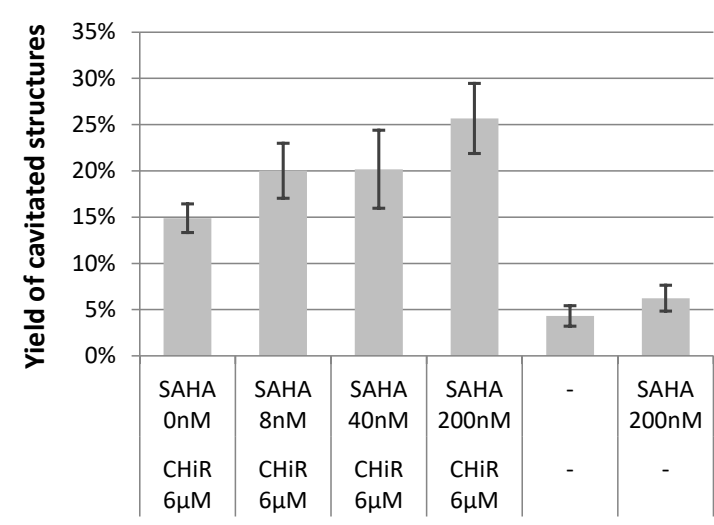

C

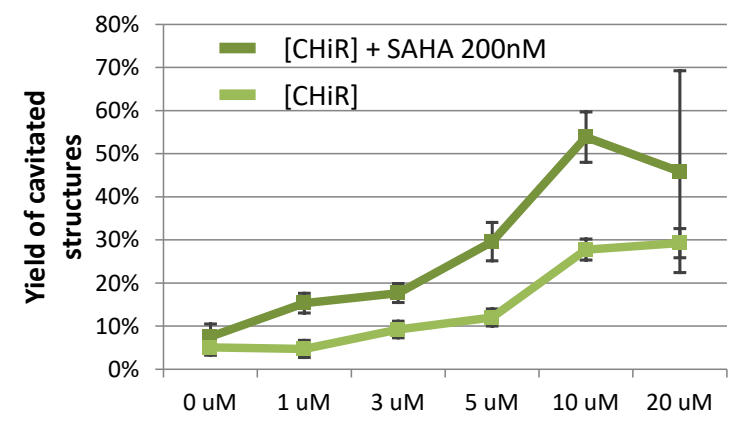

B
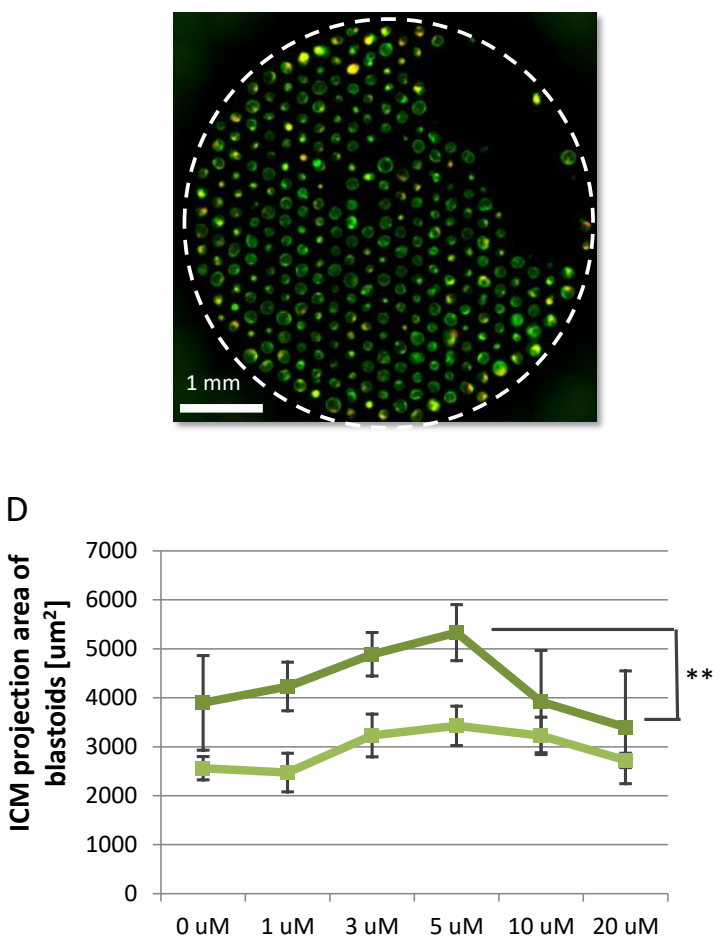

Figure 11: The yield of cavitated blastoids is CHiR and SAHA concentration dependent. (A) Dose response for SAHA; higher concentrations lead to an increased yield of cavitated structures. (B) Montage image covering a full microwell array with fluorescently labeled structures, indicating the yield of $10 \mu \mathrm{M}$ CHiR with $200 \mathrm{nM}$ SAHA culture conditions. (C) Yield of cavitated structures as a function of CHiR concentration; $10 \mu \mathrm{M}$ CHiR with $200 \mathrm{nM}$ SAHA showed the highest consistent yield. (D) Projection area of the ICM within blastoids as a function of CHiR concentration. The ICM projection area of blastoids decreases with concentrations of $10 \mu \mathrm{M} \mathrm{CHiR}$ and higher. This decrease is emphasized with the addition of $200 \mathrm{nM}$ SAHA. ${ }^{* *}$ denotes $\mathrm{p}<0.01, \mathrm{n}=6$ wells, ANOVA with Bonferroni's multiple comparison test, 12 ES cells were seeded per microwell.

\section{SAHA's effect on cavitation scales with the size of the ICM}

While blastoid formation is triggered by activating canonical Wnt signaling (Figure 11), the blastoid formation rate is dependent on the size of the ICM (Figure 7). To understand the mechanism of SAHA, the yield of blastoid formation was measured while titrating the average number of ES cells with and without supplementing 200nM of SAHA. Clearly, when ES cells were absent or very few (an average of two cells per microwell), the yield of blastoid formation was similar for both the control and SAHA (Figure 12A). This indicates that there is no preferential 
formation of blastoids in microwells where only TS cells reside, thus the ES compartment appears critical in propagating the effect of SAHA. However, other reasons for the fact that trophoblastic vesicles do barely form without an ICM cannot be excluded yet. For example, there may be a requirement for cell-cell contact or TS cells need to a cellular substrate in the form of a spherical cell cluster to form a polarized monolayer onto.

When the ES cell number was increased the yield with SAHA increased correspondingly, following the same trend as the control. However, relative to the control, the yield of blastoids gradually increased from 1.57x higher than the control at 6 ES cells, to 2.47x higher at 10 ES cells, and up to $3.27 x$ higher at 14 ES cells (Figure 12B). This suggests that the molecular mechanism of SAHA acts via the embryonic compartment.

It can be seen in Figure $12 \mathrm{C}$ that the size of the ICM was equal to the control. This suggests that proliferation of the ICM cells was not affected by SAHA, Also, it suggests that there is no preferential cavitation in microwells where the exact optimal number of 6 ES cells was seeded. Obviously, the chance lowers of finding 6 ES cells in a microwell when increasing the average ES cell seeding number. Similar to the ICM, the overall size of blastoids did not change under influence of SAHA (Figure 12D). Notably, blastoids cultured with SAHA were found to be more circular (Figure 12E).

An intrinsic property of stochastic seeding of low ES cell numbers onto the microwell array is that in a portion of the microwells no cell will settle. It can be seen in Figure $12 \mathrm{~F}$ that there is no increased incidence of empty blastoids in SAHA conditions compared to the control as the lines are identical. This indicates that SAHA does not augment ICM cell death within blastoids.

The number of fluorescent speckles observed outside the ICM (the ICM was defined as the largest embryonic compartment) was measured and classified as the number of segregated islands of embryonic origin (Figure 12G). These were observed to be single cells, potentially migrating, or a group of cells up to the size of the major embryonic compartment (artificial twinning). Adding SAHA to the blastoid culture led to a higher number of segregated islands. , which could indicate that there is a higher degree of cell motility.

Another possibility, however, is that the higher number of fluorescent speckles are cellular remnants from dead cells, which is a characteristic of cells comprising an H2B-RFP reporter. This would suggest there is simply more cellular debris in blastoids formed including SAHA. A small number of dead cells could already lead to such speckles while it does not severly affect the size of the ICM. In conclusion, SAHA appears to affect TS cells and renders them receptive for signals from the embryonic compartment which regulates trophectoderm development. 
A

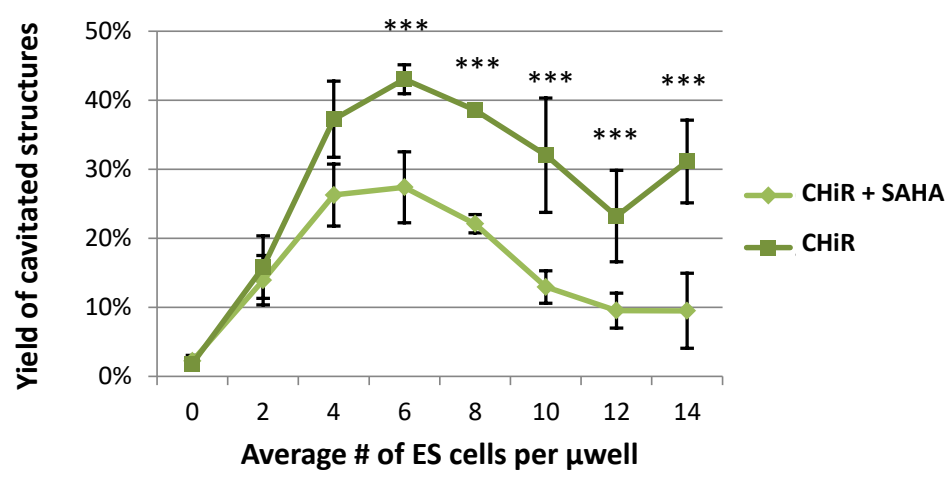

B

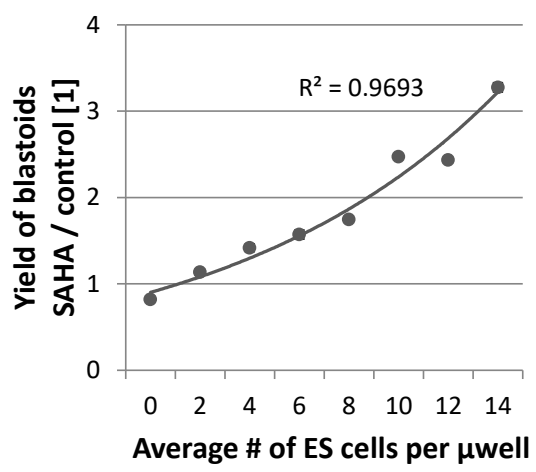

C

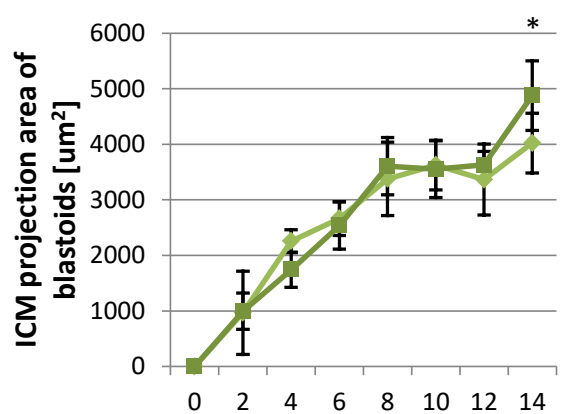

Average \# of ES cells per $\mu$ well

$\mathrm{F}$

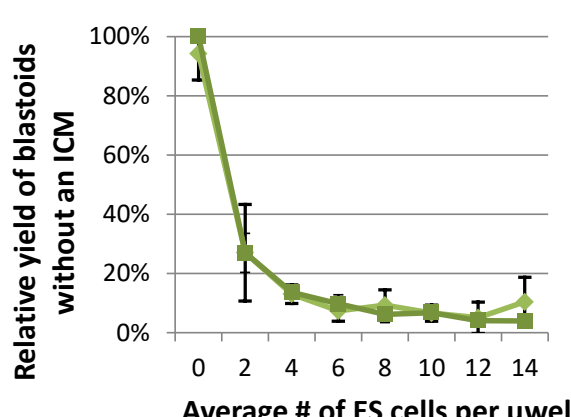

D

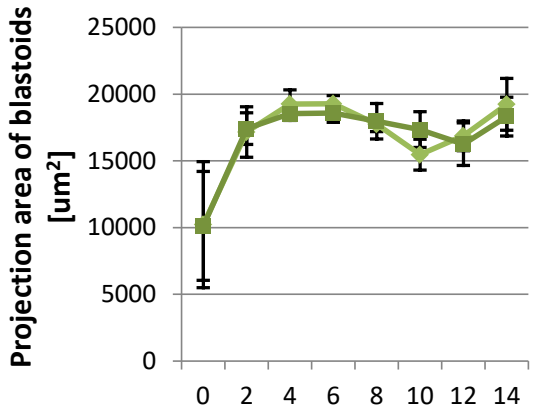

Average \# of ES cells per $\mu$ well

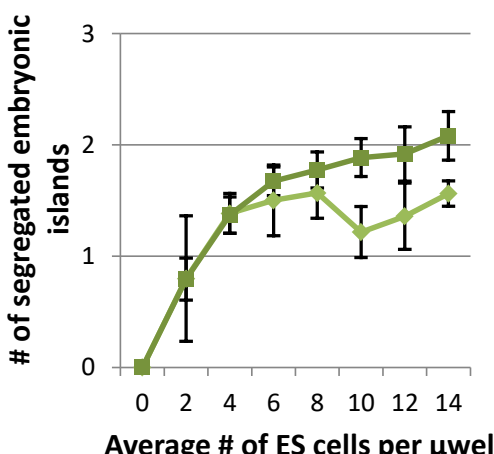

E
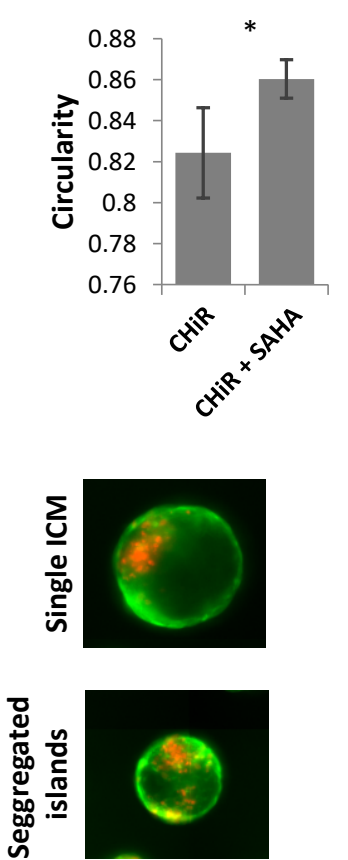

Figure 12: SAHA scales with the size of the ICM. (A) The blastoid formation rate versus the average seeding number of ES cells. The effect of SAHA was dependent on the presence of an ICM. (B) The blastoid formation rate of SAHA compared to control amplified with increasing seeding number of ES cells. (C) The projection area, as a proxy for the size of the ICM, was not affected by SAHA. (D) The overall projection area, as a proxy for blastoid size, was not affected by SAHA. (E) Circularity measurements of blastoids formed using 6 ES cells showed more circular blastoids in the presence of SAHA. (F) The yield of blastoids without an ICM showed similar values for SAHA and the control. (G) The fragmentation of the ICM measured as the number of embryonic islands segregated from the major embryonic compartment. Introducing SAHA elicited a higher number of segregated islands. 


\section{SAHA amplifies Wnt signaling}

The addition of CHiR leads to selective inhibition of GSK3, which in turn suppresses $\beta$-catenin degradation and thus activates Wnt signaling ${ }^{120}$. Activating Wnt signaling in blastoids induced cavitation, as was shown previously (Figure 11C). To investigate the role of the Wnt signaling transduction pathway in SAHA-mediated cavitation two chemical antagonists of Wnt signaling were administered on top of Wnt activation by CHiR at Oh of culture. The first one is XAV939, which stabilizes Axin and thereby stimulates the degradation of $\beta$-catenin ${ }^{121}$. The second one is IWP2, which is an inhibitor of Wnt processing through inactivation of Porcupine. When this protein is inactivated the enzyme $\mathrm{O}$-acyltransferase is not encoded and Wnt ligand secretion is inhibited ${ }^{121}$. Both of these Wnt antagonists transiently and reversibly suppress Wnt signaling.

Among the tested conditions XAV939 $(10 \mu \mathrm{M})$ led to the largest inhibiting effect on the yield of blastoid formation, including diminishing the effect from Wnt activator $\mathrm{CHiR}$ ( $80 \%$ reduction) and the synergistic effect of CHiR and SAHA (67\% reduction, Figure 13 ). Nevertheless, the blastoid yield of $\mathrm{CHiR}+\mathrm{SAHA}$ was still higher than CHiR only when treated with XAV939 as it showed a fractional decrease, suggesting that SAHA may act downstream of Wnt (exposing Wnt target genes).

The conditions including the Wnt ligand secretion inhibitor IWP2 $(2 \mu \mathrm{M})$ resulted in an minor decrease in the yield of cavitation ( $47 \%$ reduction for $\mathrm{CHiR}$ and $23 \%$ for $\mathrm{CHiR}+\mathrm{SAHA}$ ). However, the readout of this assay was $24 \mathrm{~h}$ after refreshing $50 \%$ of the medium ( $72 \mathrm{~h}$ of culture), thus removing a portion of Wnt signal inhibitors. Time lapse observations by eye witnessed a sudden increase in cavitation in the conditions with IWP2 after refreshing medium. The effect of the Wnt antagonists on SAHA only (no CHiR) was minimal and can possibly be attributed to low levels of endogenous Wnt signals. These results indicate that paracrine Wnt signaling is involved in the yield promoting effect of SAHA on blastoid formation.

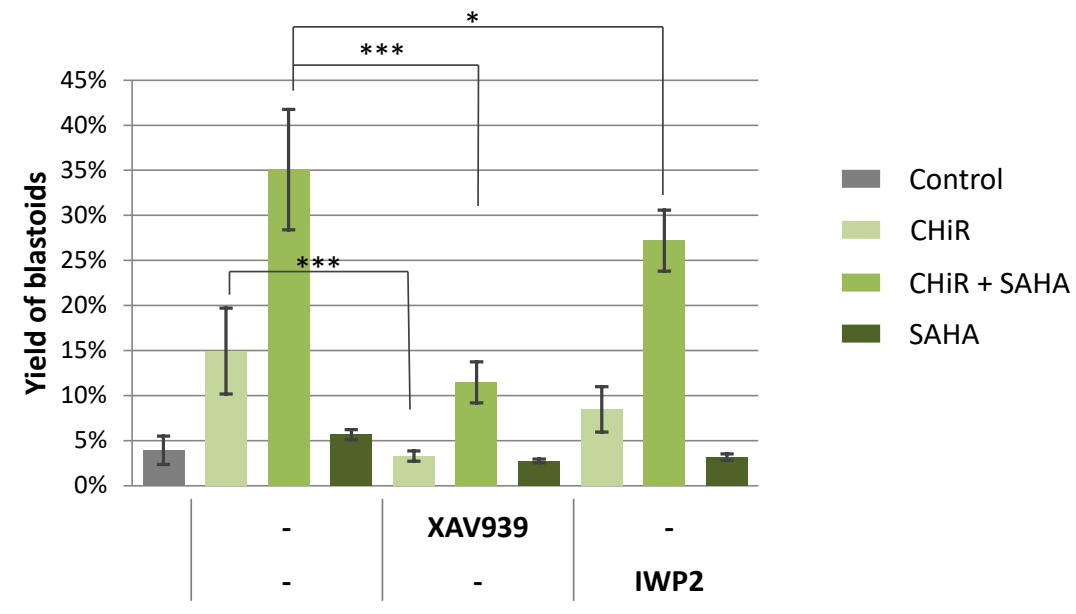

Figure 13: Inhibiting Wnt signaling attenuated the effect of SAHA on blastoid formation. Blastoids were formed with and without XAV939, an inhibitor of $\beta$-catenin-mediated Wnt signaling; and IWP2, an inhibitor of Wnt processing and secretion. In conditions with CHiR and CHiR + SAHA blastoid formation was significantly downregulated upon addition with XAV939. Addition of IWP2 also affected both conditions, but only significantly in the case of CHiR + SAHA. In the control condition no CHiR and no inhibitors were added. ${ }^{* * *}$ and $*$ denote $\mathrm{p}<0.001$ and $\mathrm{p}<0.05$ respectively, $\mathrm{n}=4$ wells, ANOVA with Bonferroni's multiple comparison test. 
Altogether, and consistent with the results from SAHA (Figure 12), Wnt signaling is essential for recapitulating the morphogenetic process of cavitation in this biofabricated model of preimplantation development, the blastoid. In the aforementioned section, SAHA was shown to synergize with the Wnt Pathway to enhance the cavitation rate. Here, adding the $\beta$-catenin transduction inhibitor XAV939 was shown to reduce the cavitation rate of $\mathrm{CHiR}$ and $\mathrm{CHiR}+\mathrm{SAHA}$ to a similar relative extend. This indicates canonical / $\beta$-catenin Wnt signaling to be the dominant form of transducing Wht signals which lead to cavitation, in both $\mathrm{CHiR}$ and CHiR + SAHA conditions. Considering the HDAC inhibiting effect of SAHA that results in relaxation of the chromatin structure, this could facilitate a range of factors to access the DNA causing transcriptional activation ${ }^{122}$. Apart from altering the chromatic folding structure, SAHA and other HDAC inhibitors have shown to acetylate proteins involved in development such as NF-KB, ER $\alpha$, GATA, stat and, importantly, the Wnt target TCF ${ }^{123}$. Acetylation of TCF can promote its retention in the nucleus and enhance activation of Wnt target genes when forming a complex with its coactivator $\beta$-catenin ${ }^{124}$. As such, SAHA may directly amplify and sustain $\beta$-catenin Wnt signaling in the nucleus, leading to enhanced cavitation rates in blastoids.

\section{SAHA affects the pluripotency of the embryonic compartment}

The effect of SAHA on blastoid formation morphology was described in Figure 12 by using spatial descriptors based on fluorescence images. In addition to this valuable information, HCS can also be employed to extract information about genetic activity within groups of cells. Here, embryonic stem cells with a knock-in allele of a TdTomato reporter for Nanog were used to form blastoids and in turn to measure the relative fluorescence intensity over time (Figure 14).

Upon culture with SAHA, the average Nanog fluorescence reporter intensity was shown to reduce over time, both in blastoids and non-cavitated structures (Figure 14). This suggests that SAHA negatively affects Nanog expression levels and thus affects the pluripotency of the embryonic compartment. Whether this is detrimental for the developmental potency of blastoids is yet uncertain, indicators of later stage embryonic development may give clues here.
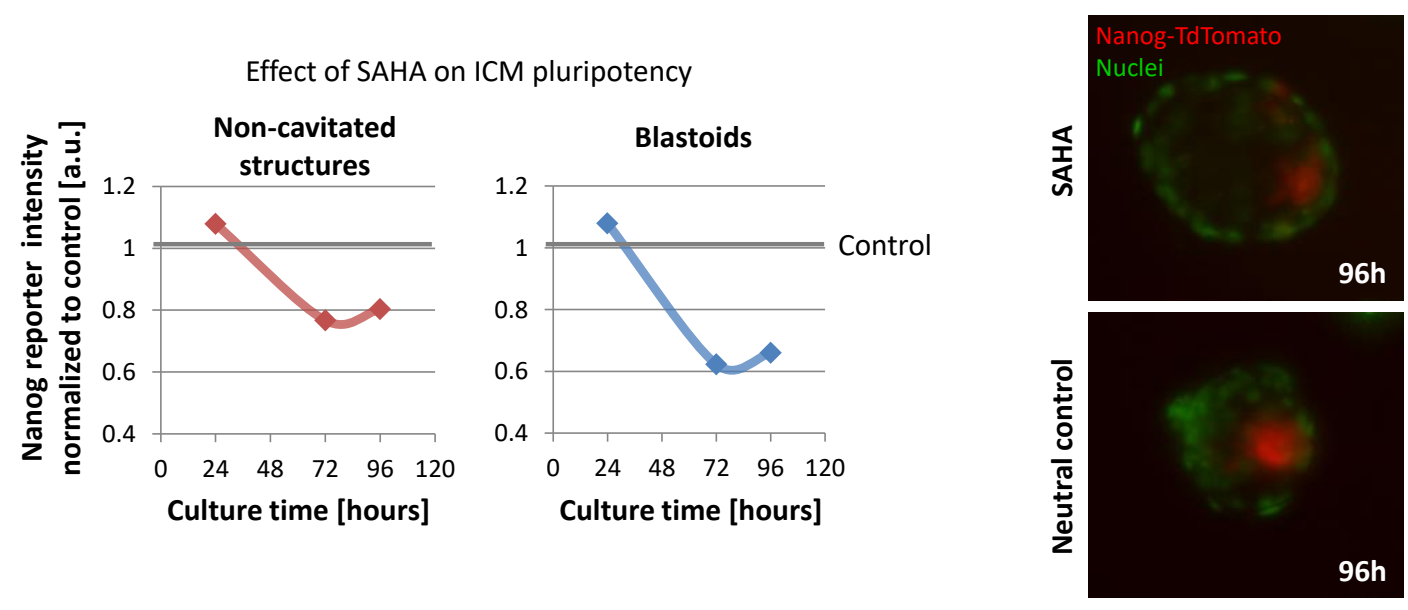

Figure 14: SAHA affects Nanog-TdTomato reporter intensity. The fluorescence intensity of the embryonic compartment in blastoids and in non-cavitated structures reduces over time upon culture with 200 nM SAHA (normalized to neutral DMSO control). Displayed are two fluorescence images representative of the blastoids with corresponding conditions. 


\section{Effect of SAHA on blastoid cavitation is maintained via TSC exposure}

In order to avoid a negative impact from SAHA on the pluripotency of the ICM and, concomitantly, retain the improved effect of SAHA on the yield of blastoids that form, it is hypothesized that blastoid culture could benefit from an indirect approach of applying SAHA. As the results of previous experiments strongly indicated, SAHA renders the trophoblast more receptive for Wnt signals, which may either be self-enforced autocrine signals in the trophoblast or paracrine signals from the ICM.

Here we employed an approach of conditioning TS cells with SAHA during 2D expansion. TS cells were expanded with and without 200nM SAHA for 48 hours, and using mEF-conditioned medium to exclude the presence of potential SAHA-stimulated paracrine factors from feeder cells. TS cells showed normal morphology and no signs of altered proliferation speed in the 2D expansion phase (Figure $15 \mathrm{~A}$ ). TS cells were harvested and used for blastoid culture. When SAHA pre-treated TS cells were used for blastoid formation the average yield was increased with 69\% (Figure $15 \mathrm{~B}$ ), although not statistically significant. Yet, this illustrates that TS cells can be pre-treated with SAHA and retain their capacity for enhanced cavitation.

Interestingly, administering SAHA on top of blastoid culture using pre-treated TS cells even further pushed up the yield of blastoid formation, which suggests higher SAHA concentrations during TS expansion may be used. Of higher importance, the yield of blastoids formed using pre-treated TS cells (10.8\%) was almost similar to that of blastoids formed using normal TS cells and supplemented with SAHA during blastoid culture $(12.8 \%$, no statistical significant difference). Of note, in this specific experiment the overall yield of blastoid formation was comparably low ( $12.8 \%$ in the SAHA condition versus at least $25 \%$ in other experiments).

These results confirm that SAHA promotes cavitation by acting directly on TS cells instead of an indirect mechanism via the embryonic compartment. In conclusion, and considered from an overarching perspective, conditioning TS cells can positively impact the developmental potency of blastoids.

A

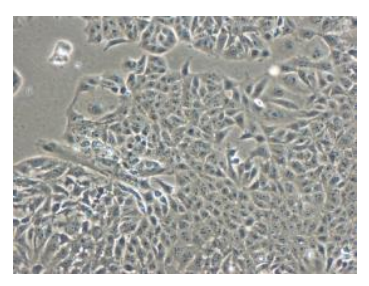

DMSO control

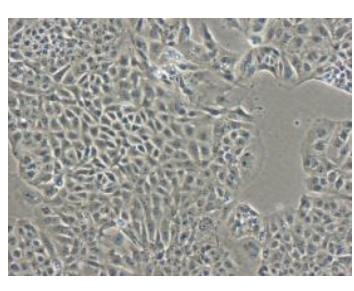

SAHA [200nM]
B

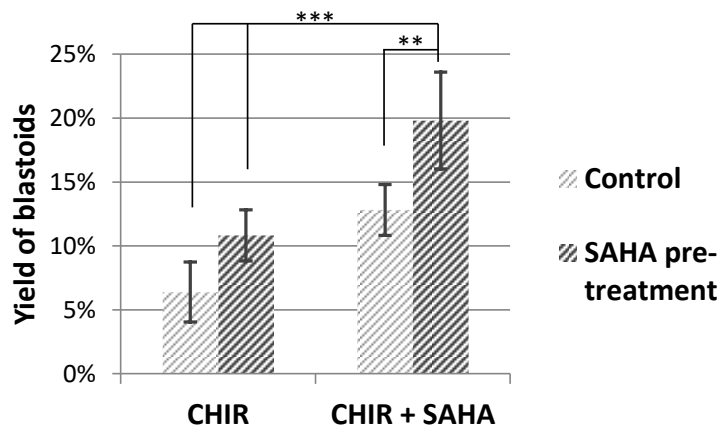

Figure 15: Pre-treating TS cells with SAHA boosts cavitation. (A) Phase-contrast images of expanding TS cells after 48 hours showed no considerable effect of 200nM SAHA on cell morphology or proliferation. (B) Expanding TSCs for 48 hours with 200nM SAHA increased cavitation yield when blastoids were formed. Cavitation increased even further when blastoid culture medium was supplemented with 200nM SAHA. Samples were fixated at 96 hours (24h EB formation $+72 \mathrm{~h}$ culture) $(10 \mu \mathrm{M}$ CHIR, 200nM SAHA, ANOVA with Bonferroni's multiple comparison test, $\mathrm{n}=6$ wells). 


\section{SAHA has a minimal effect on trophoblast identity}

To verify the suitability of using SAHA as a cavitation promoting compound, a small genetic study was done to complement the morphological read outs from previous experiments. Two important transcription factors defining the identity of TS cells are CDX2 and Eomes. Here, a qPCR analysis was performed to measure the mRNA expression levels of these genes in TS cells cultured in mEFconditioned medium and exposed to different concentrations of SAHA for 24 and $48 \mathrm{~h}$. Exposing TS cells to SAHA for 24 hours did not affect gene expression of CDX2 and Eomes (Figure 16A). Prolonging exposure till $48 \mathrm{~h}$ slightly lowered CDX2 levels (16\%) and Eomes levels (30\%), however, these small fold changes were not statistically significant (Figure 16B). Moreover, others have shown by immunostaining that $1 \mu \mathrm{M}$ of SAHA did not affect CDX2 levels in mouse blastocysts ${ }^{118}$. Altogether, it is unlikely that an exposure of SAHA up to 48 hours does have a large impact on TS pluripotency genes.

A

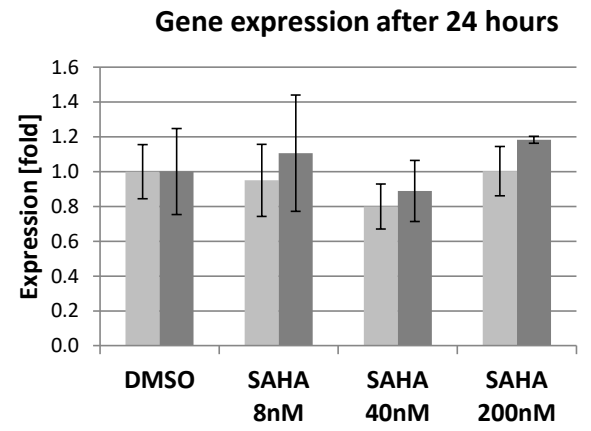

B

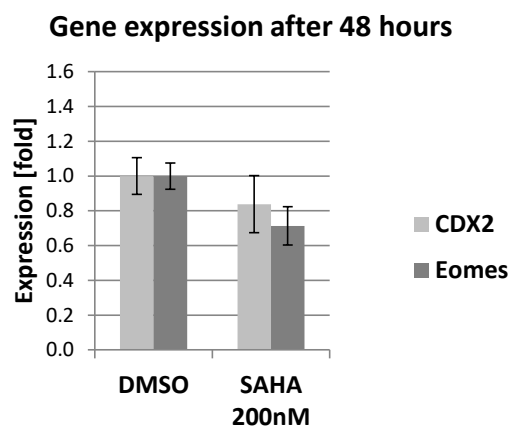

Figure 16: qPCR analysis on CDX2 and Eomes in TS cells after SAHA exposure. (A) Exposure of TSCs to SAHA for 24 hours did not result in any significant changes in mRNA levels for CDX2 and Eomes (ANOVA). (B) Extended exposure to 48 hours reduced Eomes expression with $30 \%$ ( $n=3$, n.s. ANOVA with Tukey post-test).

\section{Discussion: Molecular mechanisms of SAHA}

Concentrations of $10 \mu \mathrm{M}$ CHiR in combination with SAHA (high Wnt activation) resulted in more segregated embryonic islands or cells in blastoids, preferentially within or close to the trophectoderm. A reason may be Wnt-stimulated cell migration, as in human trophoblast cells it has been shown that migration is induced when $\beta$-catenin/ Wnt signaling is activated ${ }^{125}$. Another reason may be that ES cells were contributing in the trophectoderm by trans-differentiating into TS cells. Although TE differentiation in ES cells can be triggered by genetic overexpression of the transcription factor CDX2, Tead4 or ectopic Ras/MAPK activation ${ }^{8,126-128}$, its native plasticity towards TE conversion is disputed ${ }^{129}$. Hence, there are claims that TE differentiation from ES cells can be facilitated under Lymphoid enhancer factor 1 (LEF)-mediated Wnt signaling ${ }^{130}$. Interestingly, LEF1 expression is regulated by LIF and BMP, which are both secreted by TS cells and the trophectoderm. Taking these factors into account makes the possibility of ES cells contributing to the trophectoderm a plausible hypothesis. Especially when supplementing SAHA, which is known to facilitate reprogramming ${ }^{118}$ and was shown to boost Wnt signaling in blastoids. 


\section{EGF and FGF (MAPK) signaling downregulate cavitation in SAHA conditions}

Various growth factors that are potentially important in preimplantation development and TS and ES cell identity were tested for their effect on blastoid formation with SAHA. The growth factors HB-EGF, FGF4, IL6, TGF- $\beta 1$ and IGF2 were added in different concentrations at the time of seeding TS cells ( 0 hours of blastoid development). The results show that activation of FGF and EGF signaling reduced the blastoid formation yield in the presence of SAHA (EGF showed a reduction around 32\%, Figure 17A). Activation of insulin signaling by IGF2 also reduced the yield, however not statistically significant. Both factors signal through a receptor tyrosine kinases (RTK) which activates the MAPK pathway, known to play an important role in cell lineage specification during embryonic development ${ }^{131}$. Interestingly, only the yield was affected and not the size of blastoids (Figure 17B), indicating that EGF and IGF2 affect the initiation of cavitation. Alternatively, low concentrations of TGF- $\beta 1$ reduced the yield of blastoids and their size $(p<0.001)$.

\begin{tabular}{c|ccc}
$n G / m L$ & [low] & [medium] & [high] \\
\hline HB-EGF & 17 & 50 & 150 \\
FGF4 & 2 & 10 & 50 \\
IL6 & 1 & 5 & 25 \\
TGFb1 & 8.3 & 50 & 300 \\
IGF2 & 5 & 30 & 180
\end{tabular}
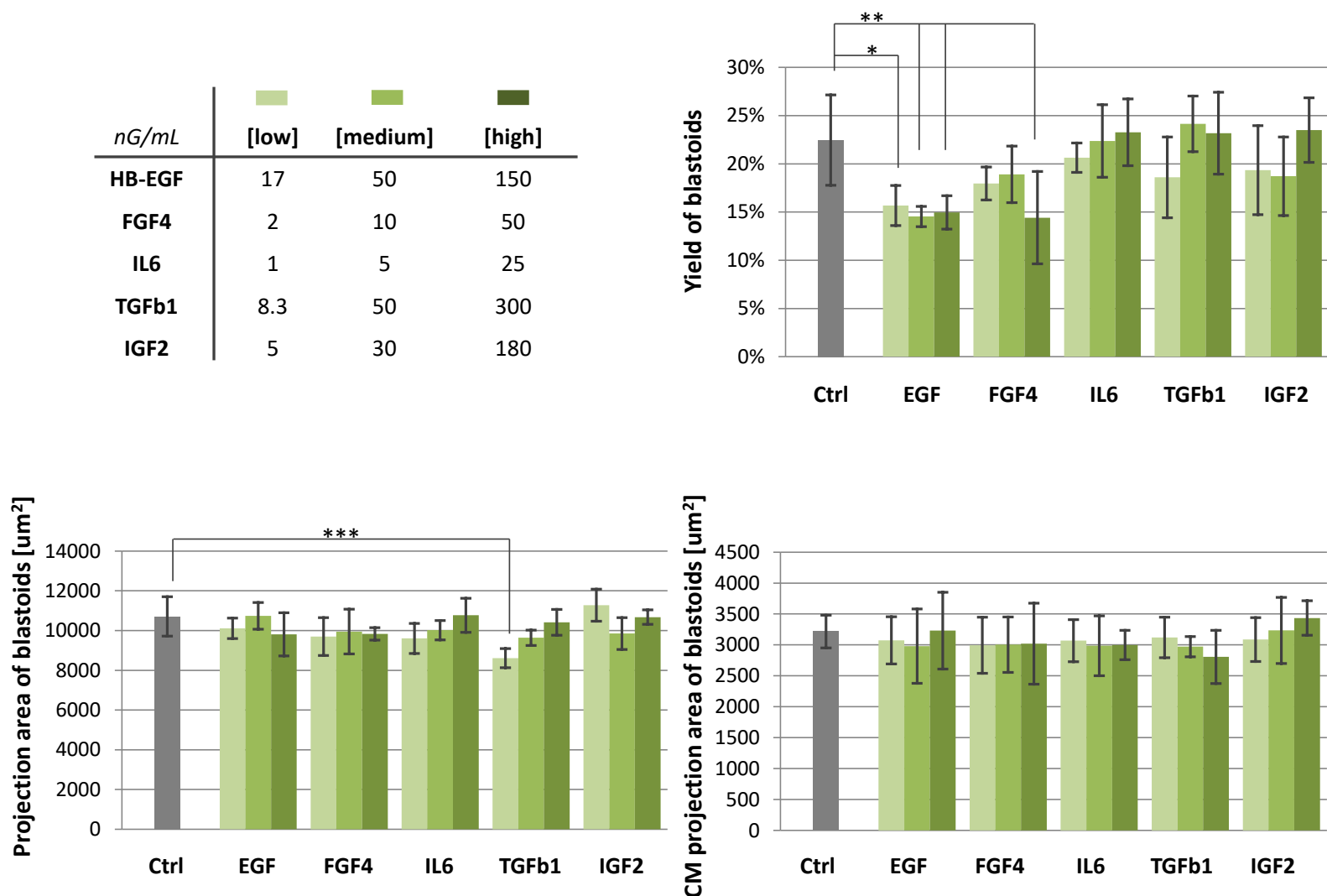

Figure 17: Effect of growth factors on blastoids cultured with SAHA. At $0 \mathrm{~h}$ of blastoid culture growth factors were administered in different concentrations in conjunction with $200 \mathrm{nM} \mathrm{SAHA}$. The effect on blastoid morphology was read-out by the projection area (as proxy for size) of the blastoid and the ICM, and the yield of blastoid formation. EGF and FGF4 showed a reduction in blastoid yield. The blastoid size was affected by low concentrations of TGFb1. The effect on the size of the ICM was limited to small deviations for TGFb1 and IGF2. 


\section{Methods}

\section{Statistical analysis}

Multiple conditions were compared using ANOVA including Bonferroni's multiple comparison test as a post-hoc test. $\mathrm{P}$ values of 0.001 , of 0.01 and of 0.05 are denoted as $* * *, * *$, and * respectively.

\section{Cell culture}

\section{Cell culture}

All cells were cultured in standard tissue culture incubators $\left(37^{\circ} \mathrm{C}\right.$ and $\left.5 \% \mathrm{CO}_{2}\right)$.

\section{Embryonic stem cells}

ES cells were trypsinized and passaged (1:20 to 1:40) every 2-3 days or when cell confluency reached approximately $80 \%$ confluency. The medium was refreshed every 2 days with twice the amount of medium or daily in the case of higher than $60 \%$ confluency.

ES-medium consisted of Dulbecco's Modified Eagle's Medium (DMEM) High Glucose (Life Technologies) supplemented with $10 \%$ fetal bovine serum (FBS selected batch, Greiner), $4 \mathrm{mM}$ Glutamax (Life Technologies), 100U/mL penicillin (Life Technologies), $100 \mathrm{mg} / \mathrm{mL}$ streptomycin (Life Technologies), $10 \mathrm{mM}$ non-essential amino acids (Life Technologies), and freshly supplemented with $0.05 \mathrm{mM}$ 2-mercaptoethanol (Life technologies), $1000 \mathrm{U} / \mathrm{mL}$ leukemia inhibitory factor (LIF, Life Technologies), $3 \mu \mathrm{M}$ CHIR99021 (GSK3 $\beta$ inhibitor, Axon Medchem) and $1 \mu \mathrm{M}$ PD0325901 (MEK/ERK inhibitor, Sigma Aldrich).

Serum-free medium (B27N2 medium) consisted of a 1:1 ratio of DMEM/F12 (Life Technologies) and Neurobasal medium (Life Technologies) supplemented with 1\% N2 and 2\% B27 (Life Technologies), $1 \mathrm{mM}$ Glutamax (Life Technologies), 1\% Non-Essential Amin Acids 100x (NEAA, Life Technologies), $100 \mathrm{U} / \mathrm{mL}$ penicillin (Life Technologies), $100 \mathrm{mg} / \mathrm{mL}$ streptomycin (Life Technologies), $0.5 \%$ bovine serum albumin (Sigma), 10mM HEPES (Fisher Scientific), $1 \mathrm{mM}$ Sodium Pyruvate (Life Technologies) freshly supplemented with $0.05 \mathrm{mM}$ 2-mercaptoethanol (Life technologies), 1000 $\mathrm{U} / \mathrm{mL}$ leukemia inhibitory factor (LIF, Life Technologies), $3 \mu \mathrm{M}$ CHIR99021 (GSK3 $\beta$ inhibitor, Axon Medchem) and $1 \mu \mathrm{M}$ PD0325901 (MEK/ERK inhibitor, Sigma Aldrich).

\section{Trophoblast stem cells}

TS wild-type and TS cells with an enhanced GFP (eGFP) reporter ${ }^{87}$ were used to form blastoids before being expanded for at least two passages. Cells were typically passaged every 3 days. Fresh medium was supplied during passaging and on day 2. Cells were either cultured on mEF cells 
$\left[10 \mathrm{k} / \mathrm{cm}^{2}\right]$ on gelatin coated dished in TS medium or on reduced Matrigel (reduced growthfactor) coated dishes in Tx-medium.

TS medium consisted of RPMI medium 1640 (Life Technologies) supplemented with 20\% fetal bovine serum (selected batch), $50 \mu \mathrm{g} / \mathrm{ml}-100 \mathrm{U} / \mathrm{ml}$ Pen/strep (Life Technologies), $1 \mathrm{mM}$ Sodium

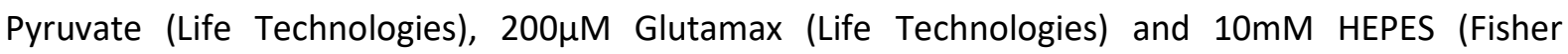

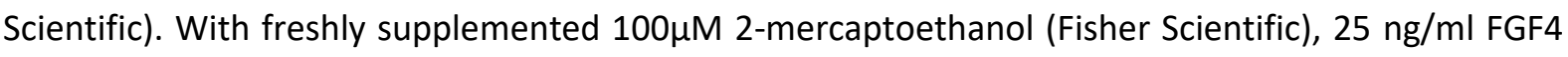
(R\&D Systems) and $1 \mu \mathrm{g} / \mathrm{ml}$ Heparin (Sigma).

Tx-medium consisted of phenolred-free DMEM/F12 (Life Technologies) supplemented with 64 $\mu \mathrm{g} / \mathrm{ml}$ L-Ascorbic-Acid-2-Phosphate (ASAP, Life Technologies), 1\% Insulin-Transferrin-Selenium (ITS G; $19.4 \mu \mathrm{g} / \mathrm{ml}$ Insulin, $10.7 \mu \mathrm{g} / \mathrm{ml}$ Transferrin, $14 \mathrm{ng} / \mathrm{ml}$ Sodium-Selenit, ThermoFisher), $200 \mu \mathrm{M}$ Glutamax (Life Technologies), $54.3 \mathrm{mg} / \mathrm{ml}$ Sodium-bicarbonate (Sigma), $1 \mathrm{mM}$ Sodium Pyruvate (Life Technologies), with freshly added $25 \mathrm{ng} / \mathrm{ml} \mathrm{FGF4} \mathrm{(R \& D} \mathrm{Systems),} 1 \mu \mathrm{g} / \mathrm{ml}$ Heparin (Sigma), $2 \mathrm{ng} / \mathrm{ml}$ TGF- $\beta 1$ (Peprotech) and optionally $2 \mu \mathrm{M}$ y27632 (Enzo Life Sciences).

\section{Blastoid culture}

Blastoid medium was comprised of serum-free B27N2 medium (described above) supplemented with $10 \%$ fetal bovine serum (FBS selected batch, Greiner), 100 $\mathrm{MM}$ 2-mercaptoethanol (Fisher Scientific), $6 \mu \mathrm{M}$ CHIR99021 (GSK3 $\beta$ inhibitor, Axon Medchem), $20 \mu \mathrm{M}$ y27632 (Enzo Life Sciences), $1 \mathrm{mM} 8 \mathrm{Br}$-cAMP (Biolog).

First, embryonic stem cells were trypisinized for 4 minutes using $0.25 \%$ Trypsin with EDTA. mEF feeder cells were depleted by differential speed of adhesion; seeding the cells twice for half an hour on TCPS dishes and harvesting the cell suspension containing a purified amount of TS cells. This was done similarly for TS cells cultured on mEF feeder cells.

Depending on the ESC line, culture medium was supplemented with $1 \mu \mathrm{M}$ ROCK inhibitor (Y27632) to facilitate EB formation within the hydrogel microwells. After 24 hours, ES-medium was removed and substituted with blastoid medium including the TS cells and soluble factors. Culture was maintained for 65 till 72 hours and fixated in $4 \%$ paraformaldehyde overnight at $4^{\circ} \mathrm{C}$.

\section{Stainings and fluorescence}

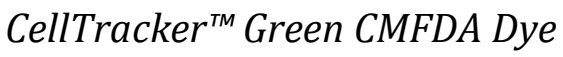

TSC were stained alive using $5 \mu \mathrm{M}$ CellTracker ${ }^{\mathrm{TM}}$ Green CMFDA (ThermoFisher scientific) Dye according manufacture's protocol. CellTracker ${ }^{\mathrm{TM}}$ Green CMFDA (5-chloromethylfluorescein diacetate) freely passes through the cell membrane where it is then enzymatically cleaved into cell membrane-impermeant products and becomes fluorescently active.

\section{Nanog-TdTomato readout}

For calculating the fluorescence intensity, CellProfiler ${ }^{132}$ was used to measure the average intensity from the median value of 3 replicates ( 3 wells), each consisting of at least 10 blastoids or moruloids. 


\section{qPCR on TSCS}

TSCs were expanded in 12 wps on gelatin in $\mathrm{mEF}$-conditioned medium for 36 hours before exposed to SAHA or DMSO ( $0.1 \%$ of total medium volume) for 24 or 48 hours.

\begin{tabular}{lll}
\hline Gene & Forward primer & Reverse primer \\
\hline CDX2 & AAAGTGAGCTGGCTGCCACACTTG & TCCATCAGTAGATGCTGTTCGTGG \\
Eomes & TGATCATCACCAAACAGGGC & ACTGTGTCTCTGAGAAGGTG \\
$\beta$-actin & TGTCGAGTCGCGTCCACC & TCGTCATCCATGGCGAACTGG \\
B2M & AAATCGTGCGTGACATCAAA & AAGGAAGGCTGGAAAAGAGC \\
\hline
\end{tabular}

Table 1: Murine qPCR primer sequences.

\section{Conclusion}

Blastoids resemble the morphological milestones that can be observed during preimplantation development of the mouse. If blastoids also genetically and functionally represent blastocysts remains to be studied. Yet, models of early embryonic development can be cultured in highthroughput using agarose hydrogel microwell arrays and are amenable to high-content screening and analysis using epifluorescence imaging, which facilitates their study in a unprecedented fashion by the ability to decouple the TS and ES compartments.

It was found that cavitation is largely driven by the embryonic compartment, probably through a combination of juxtacrine or paracrine signals, which validates this novel approach for revealing mechanisms involved in embryonic development. In conclusion, blastoids pave the way for new experimental means to study mammalian embryogenesis.

Moreover, the blastoids that are formed within the hydrogel microwell screening arrays are multicellular structures of approximately $200 \mu \mathrm{m}$ in diameter, which likely permits the extension of this microfabricated platform with high-content imaging and analysis capabilities to other multicellular models of similar size. 


\section{Discussion}

\section{Interaction between trophoblast and pluripotent niches.}

In native development, the 8-cell stage blastomeres start to compact, polarize and divide either symmetrically, yielding two outer polar cells, or asymmetrically, yielding an inner and an outer cell. The (a)symmetrical cell divisions, cell-cell contact and polarity, including intracellular molecular polarization, are central in the lineage divergence between TE and ICM cell fate and subsequent morphogenesis ${ }^{15,50}$. The method described in this chapter, however, is absent for totipotent blastomeres and instead uses cells that represent the TE and ICM cell fate and are already reinforced in their identity. Yet, this permits the formation of a structure reminiscent of a morula-stage embryo, including a polarized trophoblast that subsequently develops into a structure morphologically resembling a blastocyst. To obtain such a structure, the adequate engulfment of the pluripotent cell mass by TS cells appeared a critical initial step.

Dosing the number of pluripotent stem cells within moruloids differently modulated the yield of blastoid formation and their size. An optimum was found around 6 ES cells. In cellular aggregates formed by TS cells only, cavity formation occurred but irregularly and often including multiple fluid filled 'bubbles', similar to another study that reported the formation of trophoblast vesicles ${ }^{133}$. Considering the prerequisite of correct ES engulfment by TS cells, this suggests that other critical signals, potentially of physical or juxtacrine origin, emanate from the inner cell mass to direct trophoblast development, in addition to well-known paracrine factors such as FGF4 ${ }^{134}$. However, this does not rule out the possibility that trophoblast development, including polarization and controlled cavity formation, is mediated by particular cues from the pluripotent cell mass. To test if not any cell type at the core is sufficient to form a polarized trophoblastic monolayer and drive cavitation, a cellular mass of non-pluripotent origin should be used as a control. Nevertheless, specific soluble factors secreted by the pluripotent inner cell mass, such as FGF4, have been shown to be instrumental for proper development of the trophoblast ${ }^{135}$.

Three-dimensional cultures of trophoblast stem cells autonomously develop vascular-like spaces lined by trophoblast giant cells

\section{Alternative approaches to increase culture efficiency}

An alternative to the Poisson statistics of cells seeded in bulk on top of microwell arrays could be heralded by single-cell printing techniques ${ }^{136}$. Such systems allow depositing single cells at designated positions on a substrate material, which permit assembling specified heterotypic cell combinations applicable to blastoid formation. Several variations on cell printing techniques exist, of which the most amenable to high-throughput blastoid formation are probably the ones that generate microdroplets encapsulating single cells ${ }^{137}$. Such microdroplets can be fused to yield an accurate cell number and would allow for fusing such cell-loaded microdroplets with ones that contain a defined specification of nutrients, transcriptional modulators or growth factors 
(comparable with exosomes that allow cellular communication by transport of mRNA, miRNA and proteins between cells in vivo ${ }^{138}$ ).

\section{What would be the next step?}

\section{Post-implantation embryonic development in vitro}

Already in the mid-19 $9^{\text {th }}$ century embryology pioneers such as Karl Ernst von Baer and Robert Remak described the process of germ layer formation in chickens and introduced the terms mesoderm, ectoderm and endoderm: the fundamental precursors that give rise to all the different cell types in the body. Studying development within eggs is more accessible than in utero.

In blastocysts, after implantation into the uterus, it is very challenging to follow the subsequent developmental dynamics due to inaccessibility of the embryo. To study the direct morphogenetic processes followed by implantation, in vitro models have been developed that can recapitulate the early events of epiblast development up to the early somite stage ${ }^{139}$, as well as anterior-posterior axis and rosette formation ${ }^{140,141}$. Such advanced models necessitate the presence of extracellular components to compensate for the lack of extraembryonic tissues and maternal cues. These extraembryonic tissues provide chemical and physical signals such as integrin signalling, cell-cell adhesion and cortical tensions through matrix elasticity that help coordinate development. Anchoring EBs on a substrate mimicking the natural range of mechanical forces results in aspects reminiscent of self-organization of the three germ layers ${ }^{142}$. However, these signals do not substitute the extraembryonic compartments and therefore developmental recapitulation is limited. Conversely, the blastoid model includes both preimplantation extraembryonic tissues, which makes it an ideal candidate to explore post-implantation development in vitro. Nonetheless, this will likely be in conjunction with a maternal-based ECM embodiment to direct self-organization. The sheer high numbers of blastoids that are formed synchronously can help in the search for the essential signalling factors, by means of a screening approach for instance.

\section{Mimicking implantation in vitro}

The recent report from Zernicka-Goetz et al. demonstrated that providing hormones and ECM components in vitro that stimulate $\beta 1$-integrin signalling can regulate the polarization of epiblast cells into a rosette structure ${ }^{141}$, thereby recapitulating peri-implantation morphogenesis. This finding implicates that embryonic development can be pushed further in vitro post the implantation stage by mimicking specific assets of the extraembryonic compartments and the uterus. The basalmembrane that was provided in vitro coordinated the polarization of epiblast cells and constricted their apical conformation. In addition, supplementing the hormones $\beta$-estradiol and progesterone appeared vital. This remarkably demonstrates the in vitro organismal potential in development that aggregates of embryonic stem cells have, provided the correct specification of external cues. However, with progressing development, supplying all the soluble and physical cues necessary for appropriate patterning of the embryo becomes increasingly complex. The incorporation of a trophectoderm would therefore be desirable. For human embryos it was recently demonstrated that development could be continued in vitro up to day $10^{143}$. 
The endometrial ECM is known to modulate trophoblast adhesion during implantation and triggers peri-implantation morphogenesis of the embryo. For blastoids to implant, it is therefore important to provide the required ECM components, such as fibronectin or collagen, which binds to mouse embryos through $\beta 1$ - and $\beta 1$-class integrins on the apical surface ${ }^{144}$. Astoundingly, human cord serum was previously shown to allow embryos to develop to the somite stage in vitro including a beating heart ${ }^{145}$. In a more recent study, a polyacrylamide hydrogel with optimized elasticity and coated with type 1 rat-tail collagen was found to permit more than $80 \%$ of mouse embryos to develop beyond the blastocyst stage and form egg cylinders ${ }^{140}$. Here, the human cord serum was replaced by knock-out serum.

\section{Implantation of blastoids in vivo}

The endometrium is capable of sensing and responding to embryonic signals relating to embryo quality. For example, in bovine endometrium distinct gene signatures were identified corresponding to the origin of embryo formation: somatic cell nuclear transfer, in vitro fertilization (IVF), or artificial insemination, which related to embryo quality ${ }^{146}$. Moreover, signals emanating from developmentally viable embryos trigger the secretion of endometrial factors important for implantation ${ }^{147}$. As such, molecular mechanisms involving the endometrium contribute to the active selection of embryos for implantation. Non- invasive in vitro detection of such factors in blastoid culture and selection thereupon may increase chances of success in implantation studies.

Yet, a useful first approach would be spotting for embryos that display a correctly lined-up layer of cells positive for the PDGFRa-H2B-GFP reporter, which is a good indicator of developmental fitness of the embryonic proper. To yield a purified selection of developmentally fit blastoids, they can be 'cherry picked' from the microwell arrays and collected.

Although morphological parameters have been used with success in selecting for implantation competent blastocysts, the correct specification of the trophectoderm, including its gene expression profile is of vital importance for successful implantation and pregnancy ${ }^{148}$. The blastocyst is activated before implantation, hereby upregulating factors that coordinate the embryo-uterine dialogue such as prostaglandins and EGF signalling ${ }^{149}$. Moreover, there is a plethora of reciprocal signalling between the blastocyst and the uterus that render the endometrium receptive for implantation for example. Apart from this, the physical adhesion onto the endometrium is necessitates cell adhesion complexes within the trophectoderm such as Trophinin and E-cadherin ${ }^{150}$. The presence of such key factors should be verified and triggered within the blastoid if necessary. Alternatively, culture protocols for TS cell expansion may be optimized to obtain trophectoderm identities more similar to native trophectoderm.

\section{Acknowledgements}

Ewart Kuijk for providing the mouse H2B-RFP V6.5 ES cells. Valerie Prideaux, Jodi Garner and Janet Rossant for providing the mouse F4 TS cells, Anna-Katerina Hadjantonakis for providing the mouse H2B-GFP-PDGFRa ES cells. 


\section{References}

1. Church, D.M. et al. Lineage-specific biology revealed by a finished genome assembly of the mouse. PLoS biology 7, e1000112 (2009).

2. Mouse Genome Sequencing, C. et al. Initial sequencing and comparative analysis of the mouse genome. Nature 420, 520-562 (2002).

3. Bergsmedh, A., Donohoe, M.E., Hughes, R.A. \& Hadjantonakis, A.K. Understanding the molecular circuitry of cell lineage specification in the early mouse embryo. Genes 2, 420-448 (2011).

4. Rossant, J. \& Tam, P.P. Emerging asymmetry and embryonic patterning in early mouse development. Developmental cell 7, 155-164 (2004).

5. Niakan, K.K., Schrode, N., Cho, L.T. \& Hadjantonakis, A.K. Derivation of extraembryonic endoderm stem (XEN) cells from mouse embryos and embryonic stem cells. Nature protocols 8, 1028-1041 (2013).

6. Hadjantonakis, J.A.a.A.-K. Troika of the mouse blastocyst lineage segregation and stem cells. Curr Stem Cell Res Ther 7, 78-91 (2012).

7. Alarcon, Y.M.a.V.B. Creation of Trophectoderm, the First Epithelium, in Mouse Preimplantation Development, in Mouse Development - Results and Problems in Cell Differentiation, Vol. 55 165-184 (2012).

8. Niwa, H. et al. Interaction between Oct3/4 and $\mathrm{Cdx} 2$ determines trophectoderm differentiation. Cell 123, 917-929 (2005).

9. Skamagki, M., Wicher, K.B., Jedrusik, A., Ganguly, S. \& Zernicka-Goetz, M. Asymmetric localization of Cdx2 mRNA during the first cell-fate decision in early mouse development. Cell reports 3, 442-457 (2013).

10. Jedrusik, A. et al. Role of $\mathrm{Cdx} 2$ and cell polarity in cell allocation and specification of trophectoderm and inner cell mass in the mouse embryo. Genes \& development 22, 2692-2706 (2008).

11. Sun, J.H. et al. Differential expression of Axin1, Cdc25c and Cdkn2d mRNA in 2-cell stage mouse blastomeres. Zygote 20, 305-310 (2012).

12. Roberts, R.M., Katayama, M., Magnuson, S.R., Falduto, M.T. \& Torres, K.E. Transcript profiling of individual twin blastomeres derived by splitting two-cell stage murine embryos. Biology of reproduction 84, 487-494 (2011).

13. Marikawa, Y. \& Alarcon, V.B. Creation of trophectoderm, the first epithelium, in mouse preimplantation development. Results and problems in cell differentiation 55, 165-184 (2012).

14. Palmieri, S.L., Peter, W., Hess, H. \& Scholer, H.R. Oct-4 transcription factor is differentially expressed in the mouse embryo during establishment of the first two extraembryonic cell lineages involved in implantation. Developmental biology 166, 259-267 (1994).

15. Plachta, N., Bollenbach, T., Pease, S., Fraser, S.E. \& Pantazis, P. Oct4 kinetics predict cell lineage patterning in the early mammalian embryo. Nature cell biology 13, 117-123 (2011).

16. Chambers, I. et al. Functional expression cloning of Nanog, a pluripotency sustaining factor in embryonic stem cells. Cell 113, 643-655 (2003).

17. Mitsui, K. et al. The homeoprotein Nanog is required for maintenance of pluripotency in mouse epiblast and ES cells. Cell 113, 631-642 (2003).

18. Beck, F., Erler, T., Russell, A. \& James, R. Expression of Cdx-2 in the mouse embryo and placenta: possible role in patterning of the extra-embryonic membranes. Developmental dynamics : an official publication of the American Association of Anatomists 204, 219-227 (1995).

19. Wang, K. et al. Brg1 is required for $\mathrm{Cdx2}$-mediated repression of Oct4 expression in mouse blastocysts. PloS one 5, e10622 (2010).

20. Watson, A.J. \& Barcroft, L.C. Regulation of blastocyst formation. Frontiers in bioscience : a journal and virtual library 6, D708-730 (2001).

21. Plusa, B., Piliszek, A., Frankenberg, S., Artus, J. \& Hadjantonakis, A.K. Distinct sequential cell behaviours direct primitive endoderm formation in the mouse blastocyst. Development 135, 30813091 (2008).

22. Rossant, J., Chazaud, C. \& Yamanaka, Y. Lineage allocation and asymmetries in the early mouse embryo. Philosophical transactions of the Royal Society of London. Series B, Biological sciences 358 , 1341-1348; discussion 1349 (2003).

23. Gardner, R.L. Clonal analysis of early mammalian development. Philosophical transactions of the Royal Society of London. Series B, Biological sciences 312, 163-178 (1985). 
24. Tam, P.P. \& Loebel, D.A. Gene function in mouse embryogenesis: get set for gastrulation. Nature reviews. Genetics 8, 368-381 (2007).

25. Bedzhov, I., Graham, S.J., Leung, C.Y. \& Zernicka-Goetz, M. Developmental plasticity, cell fate specification and morphogenesis in the early mouse embryo. Philosophical transactions of the Royal Society of London. Series B, Biological sciences 369 (2014).

26. Schrode, N. et al. Anatomy of a blastocyst: cell behaviors driving cell fate choice and morphogenesis in the early mouse embryo. Genesis 51, 219-233 (2013).

27. Leung, C.Y. \& Zernicka-Goetz, M. Mapping the journey from totipotency to lineage specification in the mouse embryo. Current opinion in genetics \& development 34, 71-76 (2015).

28. Fleming, T.P. A quantitative analysis of cell allocation to trophectoderm and inner cell mass in the mouse blastocyst. Developmental biology 119, 520-531 (1987).

29. Johnson, M.H. \& Ziomek, C.A. The foundation of two distinct cell lineages within the mouse morula. Cell 24, 71-80 (1981).

30. Guo, G. et al. Resolution of cell fate decisions revealed by single-cell gene expression analysis from zygote to blastocyst. Developmental cell 18, 675-685 (2010).

31. Plusa, B. et al. Downregulation of Par3 and aPKC function directs cells towards the ICM in the preimplantation mouse embryo. Journal of cell science 118, 505-515 (2005).

32. Vinot, S. et al. Asymmetric distribution of PAR proteins in the mouse embryo begins at the 8-cell stage during compaction. Developmental biology 282, 307-319 (2005).

33. Strumpf, D. et al. Cdx2 is required for correct cell fate specification and differentiation of trophectoderm in the mouse blastocyst. Development 132, 2093-2102 (2005).

34. Ralston, A. \& Rossant, J. Cdx2 acts downstream of cell polarization to cell-autonomously promote trophectoderm fate in the early mouse embryo. Developmental biology 313, 614-629 (2008).

35. Hirate, Y. et al. Polarity-dependent distribution of angiomotin localizes Hippo signaling in preimplantation embryos. Current biology : CB 23, 1181-1194 (2013).

36. Leung, C.Y. \& Zernicka-Goetz, M. Angiomotin prevents pluripotent lineage differentiation in mouse embryos via Hippo pathway-dependent and -independent mechanisms. Nature communications 4 , 2251 (2013).

37. Cockburn, K., Biechele, S., Garner, J. \& Rossant, J. The Hippo pathway member Nf2 is required for inner cell mass specification. Current biology : CB 23, 1195-1201 (2013).

38. Rayon, $\mathrm{T}$. et al. Notch and hippo converge on $\mathrm{Cdx} 2$ to specify the trophectoderm lineage in the mouse blastocyst. Developmental cell 30, 410-422 (2014).

39. Fleming, T.P., Sheth, B. \& Fesenko, I. Cell adhesion in the preimplantation mammalian embryo and its role in trophectoderm differentiation and blastocyst morphogenesis. Frontiers in bioscience : a journal and virtual library 6, D1000-1007 (2001).

40. Moriwaki, K., Tsukita, S. \& Furuse, M. Tight junctions containing claudin 4 and 6 are essential for blastocyst formation in preimplantation mouse embryos. Developmental biology 312, 509-522 (2007).

41. Cheng, X., Ji, Z., Tsalkova, T. \& Mei, F. Epac and PKA: a tale of two intracellular cAMP receptors. Acta biochimica et biophysica Sinica 40, 651-662 (2008).

42. Manejwala, F., Kaji, E. \& Schultz, R.M. Development of activatable adenylate cyclase in the preimplantation mouse embryo and a role for cyclic AMP in blastocoel formation. Cell 46, 95-103 (1986).

43. Barcroft, L.C., Offenberg, H., Thomsen, P. \& Watson, A.J. Aquaporin proteins in murine trophectoderm mediate transepithelial water movements during cavitation. Developmental biology 256, 342-354 (2003).

44. Manejwala, F.M. \& Schultz, R.M. Blastocoel expansion in the preimplantation mouse embryo: stimulation of sodium uptake by CAMP and possible involvement of cAMP-dependent protein kinase. Developmental biology 136, 560-563 (1989).

45. Stachecki, J.J. \& Armant, D.R. Transient release of calcium from inositol 1,4,5-trisphosphate-specific stores regulates mouse preimplantation development. Development 122, 2485-2496 (1996).

46. Stachecki, J.J., Yelian, F.D., Schultz, J.F., Leach, R.E. \& Armant, D.R. Blastocyst cavitation is accelerated by ethanol- or ionophore-induced elevation of intracellular calcium. Biology of reproduction 50, 1-9 (1994).

47. Gardner, R.L. \& Rossant, J. Investigation of the fate of 4-5 day post-coitum mouse inner cell mass cells by blastocyst injection. Journal of embryology and experimental morphology 52, 141-152 (1979).

48. Shimosato, D., Shiki, M. \& Niwa, H. Extra-embryonic endoderm cells derived from ES cells induced by GATA factors acquire the character of XEN cells. BMC developmental biology 7, 80 (2007). 
49. Wicklow, E. et al. HIPPO pathway members restrict SOX2 to the inner cell mass where it promotes ICM fates in the mouse blastocyst. PLoS genetics 10, e1004618 (2014).

50. Goolam, M. et al. Heterogeneity in Oct4 and Sox2 Targets Biases Cell Fate in 4-Cell Mouse Embryos. Cell 165, 61-74 (2016).

51. Meilhac, S.M. et al. Active cell movements coupled to positional induction are involved in lineage segregation in the mouse blastocyst. Developmental biology 331, 210-221 (2009).

52. Chazaud, C., Yamanaka, Y., Pawson, T. \& Rossant, J. Early lineage segregation between epiblast and primitive endoderm in mouse blastocysts through the Grb2-MAPK pathway. Developmental cell 10, 615-624 (2006).

53. Yamanaka, Y., Lanner, F. \& Rossant, J. FGF signal-dependent segregation of primitive endoderm and epiblast in the mouse blastocyst. Development 137, 715-724 (2010).

54. Ohnishi, Y. et al. Cell-to-cell expression variability followed by signal reinforcement progressively segregates early mouse lineages. Nature cell biology 16, 27-37 (2014).

55. Kang, M., Piliszek, A., Artus, J. \& Hadjantonakis, A.K. FGF4 is required for lineage restriction and saltand-pepper distribution of primitive endoderm factors but not their initial expression in the mouse. Development 140, 267-279 (2013).

56. Schroter, C., Rue, P., Mackenzie, J.P. \& Martinez Arias, A. FGF/MAPK signaling sets the switching threshold of a bistable circuit controlling cell fate decisions in embryonic stem cells. Development 142, 4205-4216 (2015).

57. Wang, Y., Smedberg, J.L., Cai, K.Q., Capo-Chichi, D.C. \& Xu, X.X. Ectopic expression of GATA6 bypasses requirement for Grb2 in primitive endoderm formation. Developmental dynamics : an official publication of the American Association of Anatomists 240, 566-576 (2011).

58. Feldman, B., Poueymirou, W., Papaioannou, V.E., DeChiara, T.M. \& Goldfarb, M. Requirement of FGF4 for postimplantation mouse development. Science 267, 246-249 (1995).

59. Nichols, J. \& Smith, A. Naive and primed pluripotent states. Cell stem cell 4, 487-492 (2009).

60. Morris, S.A. et al. Origin and formation of the first two distinct cell types of the inner cell mass in the mouse embryo. Proceedings of the National Academy of Sciences of the United States of America 107, 6364-6369 (2010).

61. Morris, S.A., Graham, S.J., Jedrusik, A. \& Zernicka-Goetz, M. The differential response to Fgf signalling in cells internalized at different times influences lineage segregation in preimplantation mouse embryos. Open biology 3, 130104 (2013).

62. Saiz, N., Grabarek, J.B., Sabherwal, N., Papalopulu, N. \& Plusa, B. Atypical protein kinase C couples cell sorting with primitive endoderm maturation in the mouse blastocyst. Development 140, 4311-4322 (2013).

63. Morgani, S.M. \& Brickman, J.M. LIF supports primitive endoderm expansion during pre-implantation development. Development 142, 3488-3499 (2015).

64. Hogan, B.L., Cooper, A.R. \& Kurkinen, M. Incorporation into Reichert's membrane of laminin-like extracellular proteins synthesized by parietal endoderm cells of the mouse embryo. Developmental biology 80, 289-300 (1980).

65. Dickson, A.D. The disappearance of the decidua capsularis and Reichert's membrane in the mouse. Journal of anatomy 129, 571-577 (1979).

66. Solter, D., Damjanov, I. \& Skreb, N. Ultrastructure of mouse egg-cylinder. Zeitschrift fur Anatomie und Entwicklungsgeschichte 132, 291-298 (1970).

67. Damert, A., Miquerol, L., Gertsenstein, M., Risau, W. \& Nagy, A. Insufficient VEGFA activity in yolk sac endoderm compromises haematopoietic and endothelial differentiation. Development 129, 18811892 (2002).

68. Dyer, M.A., Farrington, S.M., Mohn, D., Munday, J.R. \& Baron, M.H. Indian hedgehog activates hematopoiesis and vasculogenesis and can respecify prospective neurectodermal cell fate in the mouse embryo. Development 128, 1717-1730 (2001).

69. Suwinska, A., Czolowska, R., Ozdzenski, W. \& Tarkowski, A.K. Blastomeres of the mouse embryo lose totipotency after the fifth cleavage division: expression of Cdx2 and Oct4 and developmental potential of inner and outer blastomeres of 16- and 32-cell embryos. Developmental biology 322, 133144 (2008).

70. Tarkowski, A.K. \& Wroblewska, J. Development of blastomeres of mouse eggs isolated at the 4- and 8cell stage. Journal of embryology and experimental morphology 18, 155-180 (1967).

71. Rossant, J. \& Lis, W.T. Potential of isolated mouse inner cell masses to form trophectoderm derivatives in vivo. Developmental biology 70, 255-261 (1979). 
72. Lorthongpanich, C., Doris, T.P., Limviphuvadh, V., Knowles, B.B. \& Solter, D. Developmental fate and lineage commitment of singled mouse blastomeres. Development 139, 3722-3731 (2012).

73. Li, L., Zheng, P. \& Dean, J. Maternal control of early mouse development. Development 137, 859-870 (2010).

74. Weaver, C. \& Kimelman, D. Move it or lose it: axis specification in Xenopus. Development 131, 34913499 (2004).

75. St Johnston, D. \& Nusslein-Volhard, C. The origin of pattern and polarity in the Drosophila embryo. Cell 68, 201-219 (1992).

76. Saiz, N. \& Plusa, B. Early cell fate decisions in the mouse embryo. Reproduction 145, R65-80 (2013).

77. Morris, S.A., Guo, Y. \& Zernicka-Goetz, M. Developmental plasticity is bound by pluripotency and the Fgf and Wnt signaling pathways. Cell reports 2, 756-765 (2012).

78. Burdon, T., Smith, A. \& Savatier, P. Signalling, cell cycle and pluripotency in embryonic stem cells. Trends in cell biology 12, 432-438 (2002).

79. Smith, A.G. et al. Inhibition of pluripotential embryonic stem cell differentiation by purified polypeptides. Nature 336, 688-690 (1988).

80. Hayashi, K., Lopes, S.M., Tang, F. \& Surani, M.A. Dynamic equilibrium and heterogeneity of mouse pluripotent stem cells with distinct functional and epigenetic states. Cell stem cell 3, 391-401 (2008).

81. Ying, Q.L. et al. The ground state of embryonic stem cell self-renewal. Nature 453, 519-523 (2008).

82. Marks, H. et al. The transcriptional and epigenomic foundations of ground state pluripotency. Cell 149, 590-604 (2012).

83. Toyooka, Y., Shimosato, D., Murakami, K., Takahashi, K. \& Niwa, H. Identification and characterization of subpopulations in undifferentiated ES cell culture. Development 135, 909-918 (2008).

84. Alexandrova, S. et al. Selection and dynamics of embryonic stem cell integration into early mouse embryos. Development 143, 24-34 (2016).

85. Han, D.W. et al. Epiblast stem cell subpopulations represent mouse embryos of distinct pregastrulation stages. Cell 143, 617-627 (2010).

86. ten Berge, D. et al. Embryonic stem cells require Wnt proteins to prevent differentiation to epiblast stem cells. Nature cell biology 13, 1070-1075 (2011).

87. Tanaka, S., Kunath, T., Hadjantonakis, A.K., Nagy, A. \& Rossant, J. Promotion of trophoblast stem cell proliferation by FGF4. Science 282, 2072-2075 (1998).

88. Tanaka, S. Derivation and culture of mouse trophoblast stem cells in vitro. Methods Mol Biol 329, 3544 (2006).

89. Erlebacher, A., Price, K.A. \& Glimcher, L.H. Maintenance of mouse trophoblast stem cell proliferation by TGF-beta/activin. Developmental biology 275, 158-169 (2004).

90. Kubaczka, C. et al. Derivation and maintenance of murine trophoblast stem cells under defined conditions. Stem cell reports 2, 232-242 (2014).

91. Ohinata, Y. \& Tsukiyama, T. Establishment of trophoblast stem cells under defined culture conditions in mice. Plos one 9, e107308 (2014).

92. Hayakawa, K., Himeno, E., Tanaka, S. \& Kunath, T. Isolation and manipulation of mouse trophoblast stem cells. Current protocols in stem cell biology 32, 1E 4 1-32 (2015).

93. Kunath, T. et al. Imprinted $\mathrm{X}$-inactivation in extra-embryonic endoderm cell lines from mouse blastocysts. Development 132, 1649-1661 (2005).

94. Cho, L.T. et al. Conversion from mouse embryonic to extra-embryonic endoderm stem cells reveals distinct differentiation capacities of pluripotent stem cell states. Development 139, 2866-2877 (2012).

95. Niakan, K.K. et al. Sox17 promotes differentiation in mouse embryonic stem cells by directly regulating extraembryonic gene expression and indirectly antagonizing self-renewal. Genes \& development 24, 312-326 (2010).

96. Abe, K. et al. Endoderm-specific gene expression in embryonic stem cells differentiated to embryoid bodies. Experimental cell research 229, 27-34 (1996).

97. Coucouvanis, E. \& Martin, G.R. Signals for death and survival: a two-step mechanism for cavitation in the vertebrate embryo. Cell 83, 279-287 (1995).

98. van den Brink, S.C. et al. Symmetry breaking, germ layer specification and axial organisation in aggregates of mouse embryonic stem cells. Development 141, 4231-4242 (2014).

99. ten Berge, D. et al. Wnt signaling mediates self-organization and axis formation in embryoid bodies. Cell stem cell 3, 508-518 (2008).

100. Solter, D. \& Knowles, B.B. Immunosurgery of mouse blastocyst. Proceedings of the National Academy of Sciences of the United States of America 72, 5099-5102 (1975). 
101. Dyce, J., George, M., Goodall, H. \& Fleming, T.P. Do trophectoderm and inner cell mass cells in the mouse blastocyst maintain discrete lineages? Development 100, 685-698 (1987).

102. Gardner, R.L. \& Nichols, J. An investigation of the fate of cells transplanted orthotopically between morulae/nascent blastocysts in the mouse. Hum Reprod 6, 25-35 (1991).

103. Staff, P.O. Correction: establishment of trophoblast stem cells under defined culture conditions in mice. PloS one 10, e0121167 (2015).

104. Kono, K., Tamashiro, D.A. \& Alarcon, V.B. Inhibition of RHO-ROCK signaling enhances ICM and suppresses TE characteristics through activation of Hippo signaling in the mouse blastocyst. Developmental biology 394, 142-155 (2014).

105. Duan, X. et al. ROCK inhibition prevents early mouse embryo development. Histochemistry and cell biology 142, 227-233 (2014).

106. Riento, K. \& Ridley, A.J. Rocks: multifunctional kinases in cell behaviour. Nature reviews. Molecular cell biology 4, 446-456 (2003).

107. Watanabe, K. et al. A ROCK inhibitor permits survival of dissociated human embryonic stem cells. Nature biotechnology 25, 681-686 (2007).

108. Davies, S.P., Reddy, H., Caivano, M. \& Cohen, P. Specificity and mechanism of action of some commonly used protein kinase inhibitors. The Biochemical journal 351, 95-105 (2000).

109. Enserink, J.M. et al. A novel Epac-specific CAMP analogue demonstrates independent regulation of Rap1 and ERK. Nature cell biology 4, 901-906 (2002).

110. Vilardaga, J.P., Jean-Alphonse, F.G. \& Gardella, T.J. Endosomal generation of cAMP in GPCR signaling. Nature chemical biology 10, 700-706 (2014).

111. Yasuda, E. et al. Development of cystic embryoid bodies with visceral yolk-sac-like structures from mouse embryonic stem cells using low-adherence 96-well plate. Journal of bioscience and bioengineering 107, 442-446 (2009).

112. Bielinska, M., Narita, N., Heikinheimo, M., Porter, S.B. \& Wilson, D.B. Erythropoiesis and vasculogenesis in embryoid bodies lacking visceral yolk sac endoderm. Blood 88, 3720-3730 (1996).

113. Copp, A.J. Interaction between inner cell mass and trophectoderm of the mouse blastocyst. I. A study of cellular proliferation. Journal of embryology and experimental morphology 48, 109-125 (1978).

114. Brison, D.R. \& Schultz, R.M. Apoptosis during mouse blastocyst formation: evidence for a role for survival factors including transforming growth factor alpha. Biology of reproduction 56, 1088-1096 (1997).

115. Aiken, C.E., Swoboda, P.P., Skepper, J.N. \& Johnson, M.H. The direct measurement of embryogenic volume and nucleo-cytoplasmic ratio during mouse pre-implantation development. Reproduction $\mathbf{1 2 8}$, 527-535 (2004).

116. Artus, J., Panthier, J.J. \& Hadjantonakis, A.K. A role for PDGF signaling in expansion of the extraembryonic endoderm lineage of the mouse blastocyst. Development 137, 3361-3372 (2010).

117. Kretsovali, A., Hadjimichael, C. \& Charmpilas, N. Histone deacetylase inhibitors in cell pluripotency, differentiation, and reprogramming. Stem cells international 2012, 184154 (2012).

118. Ono, T. et al. Inhibition of class Ilb histone deacetylase significantly improves cloning efficiency in mice. Biology of reproduction 83, 929-937 (2010).

119. Cartwright, P. et al. LIF/STAT3 controls ES cell self-renewal and pluripotency by a Myc-dependent mechanism. Development 132, 885-896 (2005).

120. MacDonald, B.T., Tamai, K. \& He, X. Wnt/beta-catenin signaling: components, mechanisms, and diseases. Developmental cell 17, 9-26 (2009).

121. Chen, B. et al. Small molecule-mediated disruption of Wnt-dependent signaling in tissue regeneration and cancer. Nature chemical biology 5, 100-107 (2009).

122. Kumagai, T. et al. Histone deacetylase inhibitor, suberoylanilide hydroxamic acid (Vorinostat, SAHA) profoundly inhibits the growth of human pancreatic cancer cells. International journal of cancer. Journal international du cancer 121, 656-665 (2007).

123. Drummond, D.C. et al. Clinical development of histone deacetylase inhibitors as anticancer agents. Annual review of pharmacology and toxicology 45, 495-528 (2005).

124. Gay, F. et al. Acetylation regulates subcellular localization of the Wnt signaling nuclear effector POP-1. Genes \& development 17, 717-722 (2003).

125. Sonderegger, S. et al. Wingless (Wnt)-3A induces trophoblast migration and matrix metalloproteinase2 secretion through canonical Wnt signaling and protein kinase B/AKT activation. Endocrinology 151, 211-220 (2010). 
126. Lu, C.W. et al. Ras-MAPK signaling promotes trophectoderm formation from embryonic stem cells and mouse embryos. Nature genetics 40, 921-926 (2008).

127. Blij, S., Parenti, A., Tabatabai-Yazdi, N. \& Ralston, A. Cdx2 efficiently induces trophoblast stem-like cells in naive, but not primed, pluripotent stem cells. Stem cells and development 24, 1352-1365 (2015).

128. Nishioka, N. et al. The Hippo signaling pathway components Lats and Yap pattern Tead4 activity to distinguish mouse trophectoderm from inner cell mass. Developmental cell 16, 398-410 (2009).

129. Hayashi, Y. et al. BMP4 induction of trophoblast from mouse embryonic stem cells in defined culture conditions on laminin. In vitro cellular \& developmental biology. Animal 46, 416-430 (2010).

130. He, S., Pant, D., Schiffmacher, A., Meece, A. \& Keefer, C.L. Lymphoid enhancer factor 1-mediated Wnt signaling promotes the initiation of trophoblast lineage differentiation in mouse embryonic stem cells. Stem Cells 26, 842-849 (2008).

131. Nichols, J., Silva, J., Roode, M. \& Smith, A. Suppression of Erk signalling promotes ground state pluripotency in the mouse embryo. Development 136, 3215-3222 (2009).

132. Carpenter, A.E. et al. CellProfiler: image analysis software for identifying and quantifying cell phenotypes. Genome biology 7, R100 (2006).

133. Rai, A. \& Cross, J.C. Three-dimensional cultures of trophoblast stem cells autonomously develop vascular-like spaces lined by trophoblast giant cells. Developmental biology 398, 110-119 (2015).

134. Nichols, J. et al. Formation of pluripotent stem cells in the mammalian embryo depends on the POU transcription factor Oct4. Cell 95, 379-391 (1998).

135. Murohashi, M. et al. An FGF4-FRS2alpha-Cdx2 axis in trophoblast stem cells induces Bmp4 to regulate proper growth of early mouse embryos. Stem Cells 28, 113-121 (2010).

136. Zhang, K., Chou, C.K., Xia, X., Hung, M.C. \& Qin, L. Block-Cell-Printing for live single-cell printing. Proceedings of the National Academy of Sciences of the United States of America 111, 2948-2953 (2014).

137. Wu, L., Chen, P., Dong, Y., Feng, X. \& Liu, B.F. Encapsulation of single cells on a microfluidic device integrating droplet generation with fluorescence-activated droplet sorting. Biomedical microdevices 15, 553-560 (2013).

138. Ludwig, A.K. \& Giebel, B. Exosomes: small vesicles participating in intercellular communication. The international journal of biochemistry \& cell biology 44, 11-15 (2012).

139. Hsu, Y.C. Differentiation in vitro of mouse embryos to the stage of early somite. Developmental biology 33, 403-411 (1973).

140. Morris, S.A. et al. Dynamics of anterior-posterior axis formation in the developing mouse embryo. Nature communications 3, 673 (2012).

141. Bedzhov, I. \& Zernicka-Goetz, M. Self-organizing properties of mouse pluripotent cells initiate morphogenesis upon implantation. Cell 156, 1032-1044 (2014).

142. Poh, Y.C. et al. Generation of organized germ layers from a single mouse embryonic stem cell. Nature communications 5, 4000 (2014).

143. Shahbazi, M.N. et al. Self-organization of the human embryo in the absence of maternal tissues. Nature cell biology 18, 700-708 (2016).

144. Schultz, J.F. \& Armant, D.R. Beta 1- and beta 3-class integrins mediate fibronectin binding activity at the surface of developing mouse peri-implantation blastocysts. Regulation by ligand-induced mobilization of stored receptor. The Journal of biological chemistry 270, 11522-11531 (1995).

145. Hsu, Y.C. In vitro development of individually cultured whole mouse embryos from blastocyst to early somite stage. Developmental biology 68, 453-461 (1979).

146. Mansouri-Attia, N. et al. Endometrium as an early sensor of in vitro embryo manipulation technologies. Proceedings of the National Academy of Sciences of the United States of America 106, 5687-5692 (2009).

147. Brosens, J.J. et al. Uterine selection of human embryos at implantation. Scientific reports 4, 3894 (2014).

148. Parks, J.C., McCallie, B.R., Janesch, A.M., Schoolcraft, W.B. \& Katz-Jaffe, M.G. Blastocyst gene expression correlates with implantation potential. Fertility and sterility 95, 1367-1372 (2011).

149. Wang, H. \& Dey, S.K. Roadmap to embryo implantation: clues from mouse models. Nature reviews. Genetics 7, 185-199 (2006).

150. Zhang, S. et al. Physiological and molecular determinants of embryo implantation. Molecular aspects of medicine 34, 939-980 (2013). 


\section{Supplementary information}
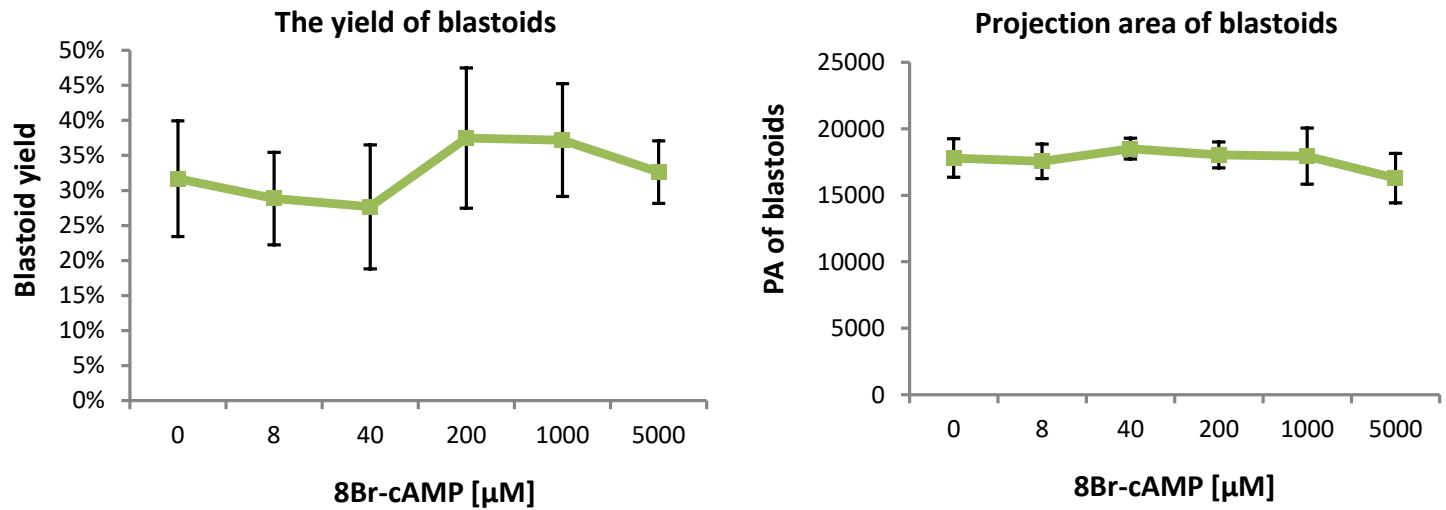

Figure SI 1: Titration of 8Br-cAMP. The blastoid yield increased from 8Br-cAMP concentrations of 200 $\mu \mathrm{M}$ and higher. The total projection area of blastoids showed no dependence on $8 \mathrm{Br}$-cAMP concentration, except for a small non-statistically significant decline at $5 \mathrm{mM}$. 

Chapter 5 


\section{Introduction}

\section{Phenotypic screening}

Small molecule screenings can roughly be divided in phenotype- or target-based screenings. Target-based drug screening is hypothesis-driven, based on disease modeling and pathway analysis, which generates candidate targets (e.g. GPCRs, kinases) for which a drug is to be found using highthroughput biochemical screening ${ }^{1}$. Conversely, phenotype-based screenings are less-biased and are set up to finding a specific phenotypic effect. Yet, they have an important additional benefit of potentially revealing unexpected targets or previously-unknown mechanisms that may provide a framework to target new biology ${ }^{2}$. An analysis of drug discovery covering the time frame between 1999 and 2008 showed that more than 50\% of first-in-class drugs were found using phenotypebased screening. This can be attributed to the fact that prior understanding of the molecular mechanism of action (MMOA) is not necessitated in phenotypic screening, which is also not mandatory for regulatory approval from for instance the US food and drug administration (FDA). Moreover, the therapeutic impact of drugs is often associated with the fact that it targets multiple molecular sites, which are harder to find using rationale target-based screenings. Also, in practice many screenings are molecular mechanism-informed phenotypic drug screenings. In the search for cancer-targeting drugs, for example, target-based selected panels of kinase inhibitors can be used in phenotype-based screening assays to identify the best candidates ${ }^{3}$. Considering the recent increase in the number of in vitro cultures that more closely replicate primary tissues and organs, including diseased ones, phenotypic screenings are expected to play an even more valuable role in approaches to identify new therapeutics and elucidate underlying mechanisms.

\section{Directed differentiation and cellular organization}

Recently, similar phenotypic screening approaches are used to interrogate stem cell biology ${ }^{4}$. One particular application of such stem cell-based screenings are directed differentiation assays. These opt in finding molecular candidates that steer progenitor cells or stem cells into a specific lineage, often with the help of fluorescent reporters ${ }^{5}$. Novel microfabricated platforms permit scaling up the level of bio-complexity in molecular screenings, for instance to multicellular models or whole organisms. These systems of hetero-cellularity include paracrine and juxtacrine signals and therefore inherently increase the signaling complexity of the model that is perturbed ${ }^{6}$. As such, screenings in the context of directing differentiation and multicellular development are typically phenotypic in nature.

Here, the agarose microwell screening array was employed to perform two small molecule screenings on 3D cellular models. The first one involves the identification of hits from a G-protein coupled receptor (GPCR) ligand library for the directed differentiation of embryonic stem (ES) cells towards the primitive endoderm lineage within embryoid bodies (EBs). The second assay includes screening a FDA-approved drug library for the identification of chemicals that enhance the engulfment of trophoblast stem (TS) cells onto small pluripotent EBs, as a step in forming blastoids. 


\section{Primitive endoderm formation and specification}

Concerted action of both intracellular transcriptional regulators and extracellular cues coordinates the cell fate decisions during early embryonic development. During preimplantation development of the mouse two rounds of distinct cell fate specification take place, one being the differentiation between the pluripotent inner cell mass and the trophoblast, and the second being the segregation from the inner cell mass into a pluripotent epiblast and the formation of primitive endoderm (PrE). The FGF/MAPK signaling pathway plays a critical role in reinforcing PrE cell fate ${ }^{7}$, but how these molecular cues are integrated to yield the correct ratio of specified cell types still remains elusive ${ }^{8}$.

\section{Transcription factor activity}

Gata6 is a potent reprogramming factor: Overexpressing the transcription factor gata6 in pluripotent stem cells initiates the upregulation of extraembryonic endoderm genes and step-wise repression of pluripotency genes Nanog and Esrrb, followed by sox 2 and oct $4{ }^{9,10}$.

Two platelet-derived growth factor receptors, PDGFR $\alpha$ and PDGFR $\beta$, can be found in higher vertebrates, which bind to at least four PDGF ligands ${ }^{11}$. Platelet-derived growth factor receptor alpha (PDGFR $\alpha$ ) was identified as one of the earliest markers of the PrE lineage. PDGFR $\alpha$ is also expressed in the PrE derivatives: the visceral endoderm (VE) and parietal endoderm (PE) ${ }^{12}$. Activation of PDGFR $\alpha$ expression in these tissues requires GATA6, which is the earliest expressed transcriptional regulator of the PrE ${ }^{13}$. Expression of gata4 and Sox17 quickly follows and both are expressed at E5.0 in the extraembryonic derivatives PE and VE. Moreover, PE cells can be reprogrammed into VE cells by a combination of laminin and BMP4 ${ }^{14}$, which exhibit reduced Gata6 expression compared to PE cells ${ }^{13}$. This implicates that VE and PE are not terminally differentiated tissues and remain amenable to cues initiating their trans-differentiation.

\section{Embryoid body culture as an in vitro model}

Differentiation of pluripotent stem cells into primitive endoderm ( $P r E)$ can be modeled in vitro by the aggregation of embryonic stem cells into embryoid bodies (EBs), which induces spontaneous extraembryonic endoderm differentiation ${ }^{15,16}$. Moreover, PrE differentiation can be induced by an exogenous pulse of Retinoic Acid (RA) ${ }^{17,18}$, which triggers the FGF signaling pathway ${ }^{18,19}$. Identical to native development, the transcription factor Gata6 is essential for both EB-mediated and RAinduced PrE differentiation ${ }^{20}$.

\section{Results}

\section{Time window of primitive endoderm differentiation in embryoid}

\section{bodies}

To identify the time window for activation of the primitive endoderm program an embryonic stem cell line was employed containing a fluorescent nuclear reporter for PDGFR $\alpha$ (PDGFR $\alpha-\mathrm{H} 2 \mathrm{~B}-$ GFP) ${ }^{12,21}$. The small retinoid retinoic acid (RA) was used as a positive control for stimulated $\operatorname{PrE}$ 
differentiation in EBs. ES cells were seeded within microwells and allowed to form EBs in ES medium supplemented with LIF for 24 hours before PrE modulating conditions were applied in a timedependent fashion. These included conditions of PrE inhibition by adding a GSK3 $\beta$ and MEK/ERK inhibitor (2i) or PrE activation by adding RA to the culture medium at 0,24 and 48 hours after EB formation (Figure 1A). After 96 hours of culture EBs were fixated using $4 \% \mathrm{w} / \mathrm{v}$ paraformaldehyde solution (PFA) and the EBs positive for PDGFRa-H2B-GFP were identified and quantified (Figure 1B). Interestingly, when $2 \mathrm{i}$ conditions were applied at 0 hours ( 24 hours after seeding ES cells), the expression of PDGFR $\alpha$-H2B-GFP was almost fully inhibited (Figure 1A). However, when 2i conditions were applied at 24 or 48 hours, there was no difference compared to the control (13 cells). Contrariwise, RA conditions led to an upregulation of PDGFR $\alpha$-H2B-GFP only when not administered from 24 hours and onwards. This indicates that there is a limited time window in which ES cells within EBs can be inhibited in their differentiation towards PrE (Figure 1C). Additionally, the results suggest that expression of the early PrE marker PDGFR $\alpha$ takes at least 48 hours from the onset of triggering differentiation. Moreover, a higher ES seeding number of 17 cells appears to result in more PDGFRa-H2B-GFP+ EBs than seeding 9 or 13 ES cells, possibly resulting from more rapidly established cell-cell junctions (collective cellular cause) or increased chances of a propagating signal originating from a single differentiating cell.

A
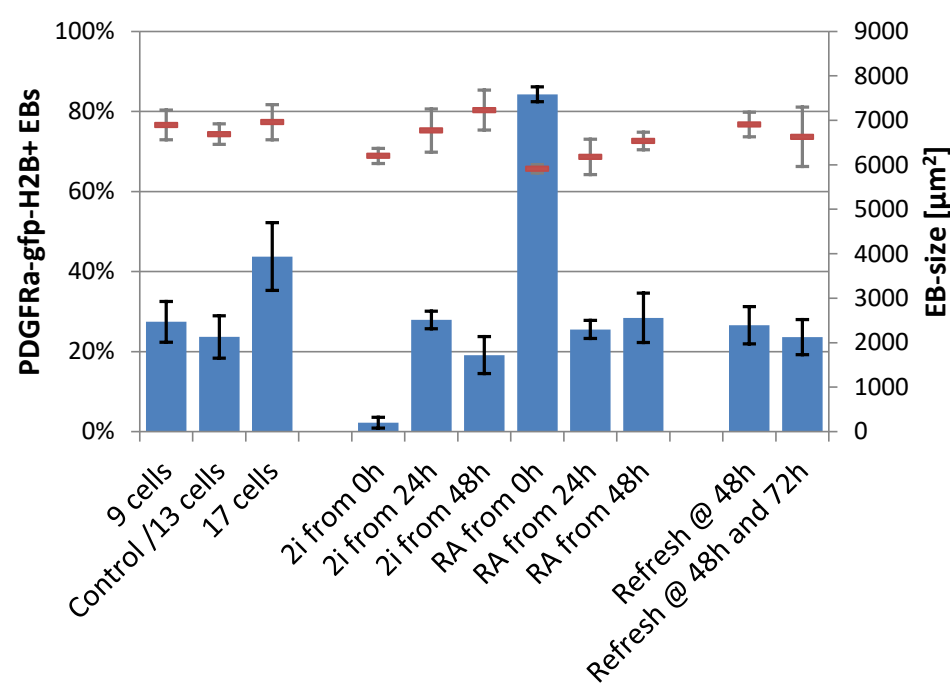

C

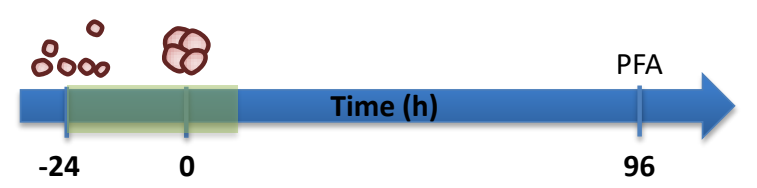

B

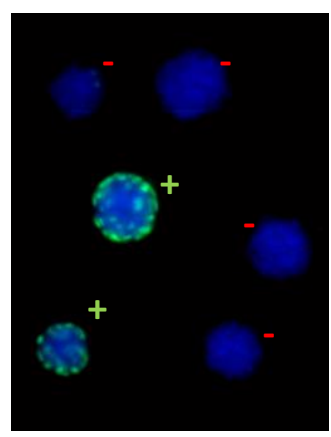

Figure 1: Time window for inhibiting PrE formation in EBs. (A) Administering inhibitors (2i) or activators (RA) of primitive endoderm differentiation in EBs at 0 hours resulted in differential yields of PDGFR $\alpha-\mathrm{H} 2 \mathrm{~B}-\mathrm{GFP}+\mathrm{EBs}$ compared to other time points. (B) Image of EBs from control conditions (13 ES cells) after 96 hours displaying the identification of EBs positive for PDGFR $\alpha-H 2 B-G F P$, based on intensity thresholding. (C) Schematic showing potential time window where differentiation of ES cells towards PrE can be modulated. Of note, time points compare with those for blastoid culture, thus Oh equals when EBs have formed 24 hours after ES cells were seeded. 


\section{Directing differentiation by screening EBs on GPCR ligands}

\section{GPCR signaling}

GPCRs are seven-transmembrane domain receptors (7TM receptors), and constitute a superfamily of diverse proteins which amplify signals from the extracellular domain into the cell. These signals include the propagation of physical signals from the outside world as well as different endogenous signals. External signals include those from odors, pheromones, taste (the chemosensory GPCRs) and photons. Most other GPCRs respond to endogenous signals like Ca2+, peptides, fatty acid derivatives, neurotransmitters or nucleotides. These signals are involved in processes encompassing behavior, development, reproduction and hormonal homeostasis ${ }^{22,23}$. All these functions associate GPCRs as the most ubiquitously present plasma membrane receptors in the cell. Moreover, most GPCRs that bind to endogenous signals are differentially expressed in cell types from different tissues. This typical feature of GPCRs, in conjunction with their high structural diversity, has made them a favorite target in the pharmaceutical industry for developing therapeutics. In fact, more than $25 \%$ of all FDA approved drugs act on GPCRs (data from 2006) ${ }^{24}$. Naturally, the repertoire of GPCRs that bind to endogenous ligands is highly preserved between mice and human, and results from about 400 genes ${ }^{22}$.

\section{cAMP-dependent and Phospholipase C signal transduction pathways}

The cascade of cellular responses after ligand binding to GPCRs is generally initiated through the activation of G-proteins, which are heterotrimeric proteins consisting of $\alpha, \beta$, and $\gamma$ subunits that can bind to and hydrolyze both the nucleotides guanosine triphosphate (GTP, 'on' state) and guanosine diphosphate (GDP, 'off' state). These then trigger second messengers with downstream activation including the mitogen-activated protein kinase (MAPK) and ROCK pathway. However, the two principal downstream transduction pathways involved in G-protein coupled receptors are cyclic adenosine monophosphate (CAMP) and Phosphoinositide 3-kinase (PI3K) ${ }^{25}$. The phospholipase $\mathrm{C}$ pathway activates the two second messengers IP3 and diacylglycerol (DAG). IP3 triggers Ca2+ signaling by releasing these ions from the intracellular calcium store in the endoplasmic reticulum, whereas DAG recruits and activates proteins of kinase C (PKC). In the CAMP-dependent transduction pathway the membrane-associated enzyme adenylyl cyclase catalyzes CAMP synthesis, which in turn leads to the activation of protein kinase $A$ and the exchange proteins activated by CAMP (EPAC).

\section{Delineating the effect of blastoid factors on PDGFR $\alpha$ expression in EBS}

To test the effect of individual blastoid culture components on the yield of PDGFR $\alpha-H 2 B-G F P$ expression in EBs, the compounds CHiR, 8Br-CAMP, y27632, TGFB1, FGF4 and IL-11 were administered to EBs (Figure 2). The results show that $\mathrm{Wnt} / \beta$-catenin activation via GSK3 $\beta$ inhibition ( 3 and $10 \mu \mathrm{M} \mathrm{CHiR}$ ) did not affect the differentiation of ES cells towards the PrE lineage within EBs. In contrast, CAMP ( $1 \mathrm{mM} 8 \mathrm{Br}$-cAMP) did increase the number of EBs positive for PDGFR $\alpha$-H2B-GFP, suggesting a potential role in extraembryonic endoderm formation for the second messenger CAMP, including its downstream signal transduction via PKA and/or EPAC.

Interestingly, all blastoid culture components together resulted in a larger positive effect on the yield of GFP+ EBs than the cumulative net effect of the individual components (Figure 2). This indicates a synergistic effect between factors in stimulating PrE differentiation within EBs. 


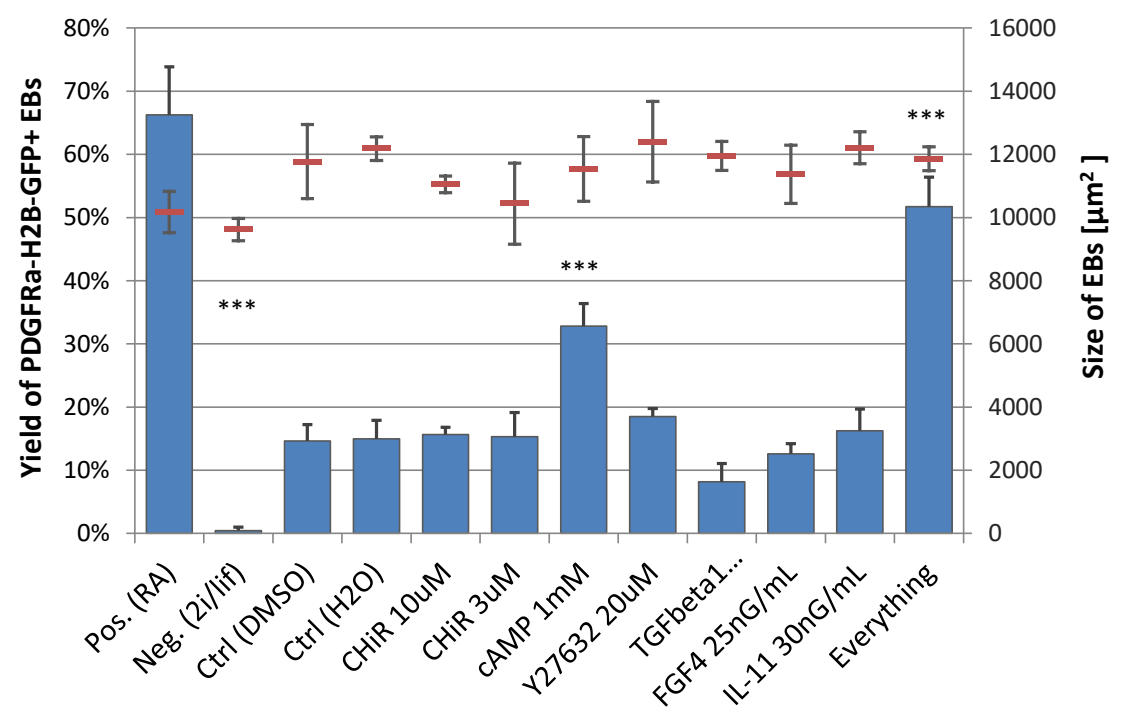

Figure 2: Delineating the effect of blastoid culture components on PDGFR $\alpha$-H2B-GFP expression in EBs. Addition of $1 \mathrm{mM}$ cAMP resulted in an increased yield of GFP+ EBs. All factors combined (everything) led to the highest yield after the positive control (RA). *** denotes a statistical significant effect of $p<0.001$ by ANOVA with Tukey's multiple comparison test among all *** conditions and compared to control (DMSO), $\mathrm{n}=4$.

\section{Screening a GPCR ligand library}

A GPCR compound screening library from SelleckChem (Catalog No. L2200), containing 264 agonists, antagonists and direct downstream effectors of GPCRs, was used to screen for the effect on PDGFRa expression in EBs (Figure 3A). The screen was performed in duplicate and in two different basal culture conditions: 1) B27N2 medium only, and 2) B27N2 medium supplemented with serum (10\% FBS) and the basic factors for blastoid formation (CHiR, 8Br-cAMP and y27632).

Firstly, the basic yield of PDGFR $\alpha-H 2 B-G F P+E B s$ was significantly higher in conditions with serum and blastoid factors, which was also observed in hit validation experiments (Figure $3 \mathrm{~B}$ and $\mathrm{C}$ ). Basic PrE formation in B27N2-only medium was low with 10 to $25 \%$ of EBs that were PDGFR $\alpha$-H2B-GFP+ (Figure $3 C$ ). The addition of serum and blastoid factors elevated basic expression (DMSO control) to around $40 \%$ (Figure $3 \mathrm{~B}$ ). This may be explained by the presence of GPCR stimulating factors in serum and of the cAMP isoform (8Br-cAMP) in blastoid culture medium. Secondly, GPCR ligand compounds displayed strong differential effects on PDGFR $\alpha$ expression when either combined with serum and blastoid factors or without (Figure 3B and C). For example, the cAMP/PKA activating B-adrenergic receptor agonist DL-adrenaline had a significant effect in blastoid medium but not in B27N2 with serum and blastoid factors (Figure $3 \mathrm{~B}$ and $\mathrm{C}$ ). The $\mathrm{CAMP}$ signal may have been saturated by the addition of $8 \mathrm{Br}$-cAMP, thus masking or diminishing the indirect effect of cAMP activation via $\beta$ adrenergic receptor binding. 


\section{Confirmation of hits}

The confirmation assay of primary hits in B27N2-only medium yielded the following adrenergic receptor agonists with a statistical significant difference of at least $p<0.01$ : DL-Adrenaline, LAdrenaline, Ritodrine ( $\beta 2$-adrenergic agonist), Isoprenaline ( $\beta 1,2$-adrenergic agonist) and Adrenalone (all at a working concentration of $20 \mu \mathrm{M}$ ). Ritodrine and Isoprenaline are $\beta$-adrenergic agonists and DL-Adrenaline is a non-selective adrenergic receptor agonist, which includes $\alpha 1, \alpha 2, \beta 1$, $\beta 2, \beta 3$ receptors. Agonist binding to $\beta$-adrenoceptors causes a rise in cAMP, which could explain the simulative effect of DL-adrenaline (290\% higher than control) and $\beta$-specific agonists (Figure 3C). Isoprenaline has an additional PDE3 inhibiting effect, thereby preventing the inactivation of CAMP. Correspondingly, the indirect cAMP activator Forskolin $(20 \mu \mathrm{M})$ and 8Br-cAMP $(100 \mu \mathrm{M})$ upregulated PDGFR $\alpha$-H2B-GFP expression in EBs with $117 \%$ and $68 \%$ respectively. Interestingly, $8 \mathrm{Br}$-cAMP appeared more effective at a concentration of $100 \mu \mathrm{M}$ than at $1 \mathrm{mM}$. This suggests that the standard concentration in blastoid culture conditions of $1 \mathrm{mM} 8 \mathrm{Br}$-cAMP could limit the full potential of cAMP/PKA stimulated PrE formation in blastoids.

The confirmation assay of primary hits in B27N2 medium including serum and blastoid factors yielded a statistical significant difference of at least $p<0.01$ for the following compounds: Betaxolol, Almotriptan, Gabapentin, Benserazide, BMY7378 and Adrenalone. These compounds act on different GPCR classes, including receptors for serotonin (5-HT), gaba and dopamine (Figure 3B). Gabepentin showed the largest additional increase on top of 8Br-cAMP (45\% increase compared to DMSO control). Almotriptan (5-HT1B/1D agonist) and Gabapentin do not show affinity with adrenergic receptors according to the Similarity Ensemble Approach ${ }^{26}$. In fact, both compounds couple with high specificity to $G_{i}$ protein coupled receptors that lead to inhibition of adenylate cyclase and lower cellular levels of cAMP, indicating that in these conditions the high supplemented levels of cAMP ( $1 \mathrm{mM}$ of $8 \mathrm{Br}$-cAMP) are disadvantageous.

Altogether, the results suggest that elevated levels of intracellular cAMP contribute to PDGFR $\alpha$ H2B-GFP expression in EBs, however, with an optimal value lower than $1 \mathrm{mM}$. 
A

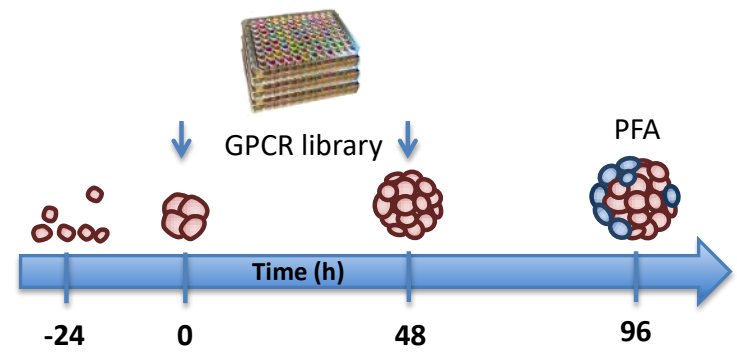

B B27N2 + FBS + blastoid factors
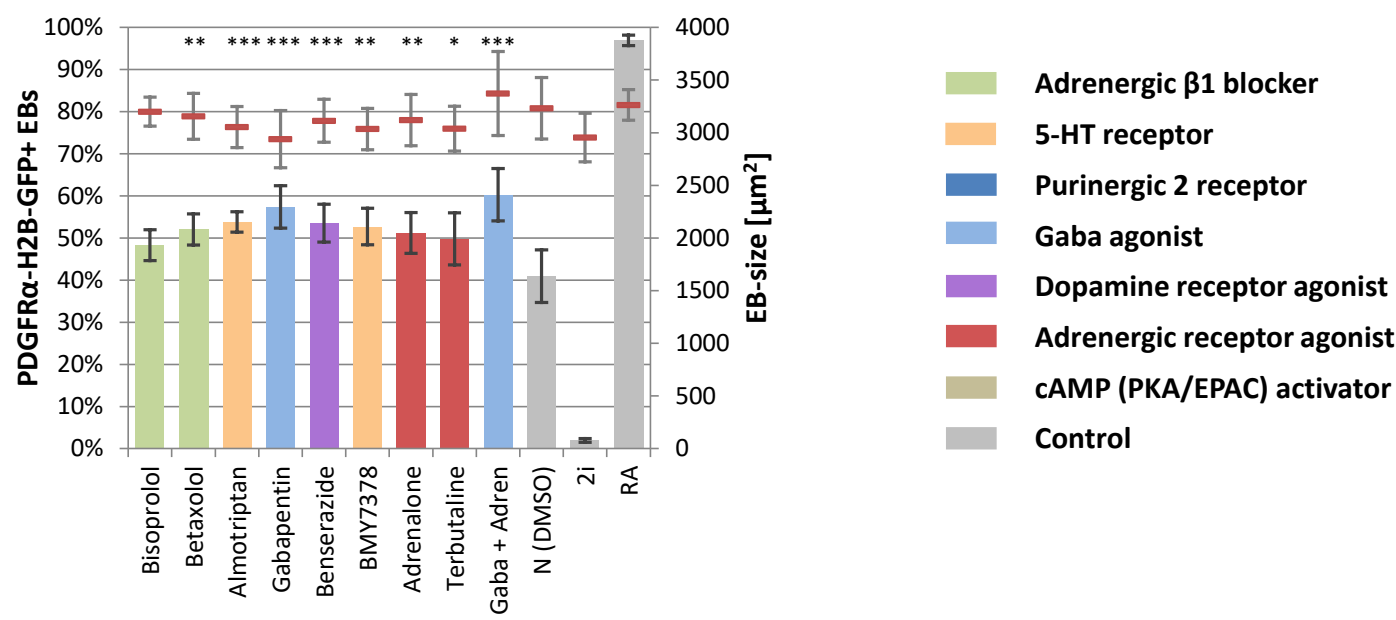

C B27N2

B27N2
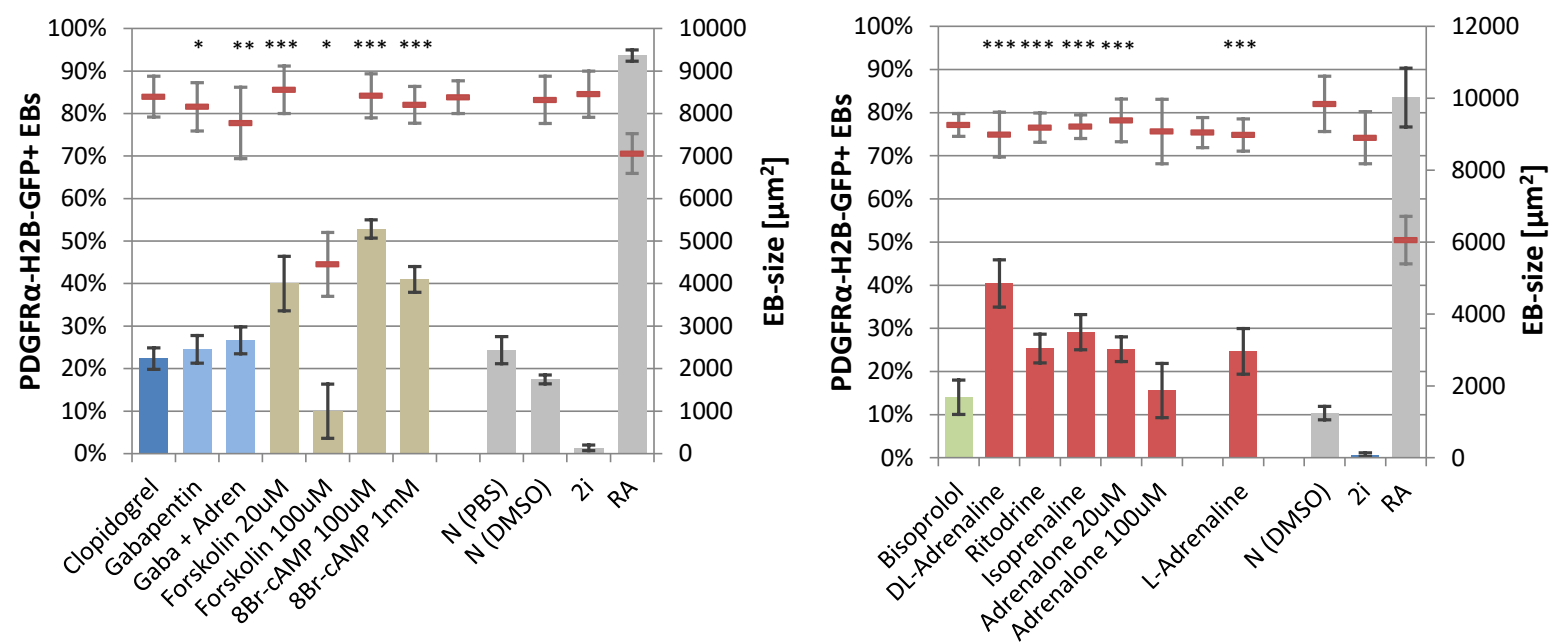

Figure 3: GPCR ligand screening on PDGFR $\alpha$-H2B-GFP expression in EBs and validation of primary hits. (A) GPCR ligand screening includes administering compounds twice at $20 \mu \mathrm{M}$ at 0 and 48 hours of culture. (B) Primary hits from the screen using B27N2 medium including serum (FBS) and the blastoid factors. (C) Primary hits from the same screen using only B27N2 medium. ${ }^{* * *},{ }^{* *}$ and ${ }^{*}$ denote a statistical significance of $p<$ $0.001, p<0.01$ and $p<0.05$ respectively, ANOVA with Tukey's post-hoc test, asterisks depict comparison with respective control. 


\section{Outlook}

Next steps will include optimizing the modulation of cAMP levels, either by directly adding $8 \mathrm{Br}$ cAMP or indirectly via $\beta$-adrenergic receptor activation. Meanwhile, hits from the EB screens were tested for their potential translation to the blastoid model, by performing a small explorative study.

\section{Priming of EBs versus direct exposure of blastoids}

Hit compounds were assessed for their translation into blastoid culture using two approaches: 1) adding the compounds directly together with ES cell seeding, hereby priming the ES cells for differentiation for a time window of 24 hours, and 2) adding the compounds simultaneously with blastoid culture factors and TS cell seeding (Figure 4A). Both strategies were employed in a preliminary assay of $n=2$ wells ( 430 microwells per well) in which blastoids were generated using a combination of ES cells containing the PDGFR $\alpha$-H2B-GFP reporter and TS cells with a cytoplasmic GFP reporter. This resulted in blastoids that showed the presence of cytoplasmic GFP in their trophoblast (TS cells) and occasionally cells positive for PDGFRa-H2B-GFP within their ICM. The total yield that describes structures both cavitated and containing PDGFR $\alpha-\mathrm{H} 2 \mathrm{~B}-\mathrm{GFP}+$ cells within their ICM, indicative of PrE formation, ranged from $2.56 \%$ to $4.90 \%$ (Figure $4 \mathrm{~B}$ and C). Although replicate numbers were low $(n=2)$, both strategies of priming and adding the hit compound Gabapentin showed an increase in yield of PDGFR $\alpha-H 2 B-G F P+$ blastoids of at least $45 \%$ (Figure 4C).

A

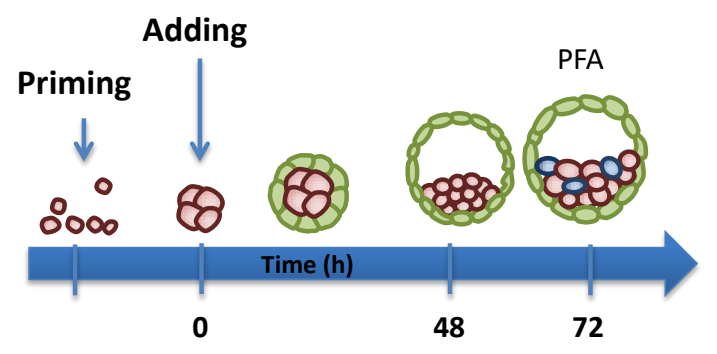

B
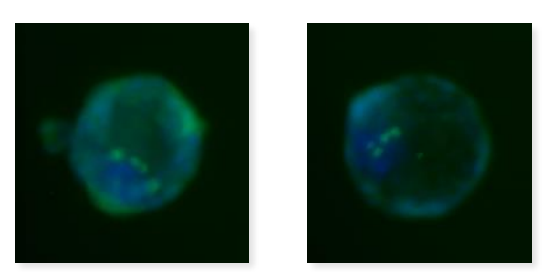

- TS-gfp and ES-PDGFRa-H2B-GFP - Nuclei

C

\begin{tabular}{|ll|lr|}
\hline Priming EBs & & \multicolumn{2}{|l|}{ Adding to blastoid culture: } \\
\cline { 3 - 3 } & $2.56 \%$ & DMSO: & $3.26 \%$ \\
Gabapentin: & $3.72 \%$ & Gabapentin: & $4.90 \%$
\end{tabular}

Figure 4: Priming and administering hit compounds for blastoid culture. (A) Two strategies of priming and adding hit compounds to stimulate PrE formation in blastoids. (B) Fluorescence images of blastoids showing a partial alignment of PDGFR $\alpha-H 2 B-G F P+$ cells. (C) Preliminary results from an assay of $n=2$ wells shows ratio of blastoids compared to all structures that contain ES cells positive for PDGFR $\alpha-\mathrm{H} 2 \mathrm{~B}-\mathrm{GFP}$, for both priming and addition of Gabapentin.

As of now, hits should be confirmed and optimized for their concentration in blastoids formed using wild-type TS cells and in high replicate numbers that allows proper statistics. Additionally, PrE identity should be validated by immunocytochemistry for markers such as gata6 and gata4 that colocalize with PDGFR $\alpha-\mathrm{H}_{2 \mathrm{~B}-G F P}{ }^{21}$, and sox17. Logically, this also applies for the epiblast and trophectoderm identity. In case hit compounds successfully translate to blastoids, the molecular mechanisms downstream of cAMP may be studied, for example using EPAC and PKA-specific small molecule activators ${ }^{27,28}$. 


\section{Small molecule screening to optimize moruloid formation}

The multicellular structure that precedes blastoid formation is one where a TS cell monolayer effectively engulfs a small population of ES cells. Such a structure, including a polarized trophoblast membrane that completely surrounds a pluripotent cell mass, morphologically resembles the late stage morula and enables the pumping of fluid inwards to form a cavitated structure ${ }^{29}$.

The yield of moruloid structures that show proper engulfment is partially dependent on soluble factors in the culture medium. In chapter 4 it was shown how high-content imaging and analysis were used to obtain quantitative data from the morphological features of these moruloids to effectively describe the engulfment efficiency. This descriptor was obtained using a method that calculates the overlap in projection area between a synthesized ring around the inner embryonic core (using an ES cell line comprising a constitutively active H2B-RFP fluorescent reporter) and the TS cell layer (using a TS cell line comprising a constitutively active cytoplasmic eGFP reporter or live staining using Celltracker green CMFDA, Chapter 4, Figure 5A, step 2). Hence, it was shown that the p160ROCK inhibitor y27632 increased the engulfment efficiency dramatically with an optimal concentration around $10 \mu \mathrm{M}$.

Here, using the same morphological descriptor as described above, a selected panel of small chemicals including ROCK inhibitors (Thiazovivin and Hydroxyfasudil) was tested to acquire an indication of the range of engulfment efficiencies that can be obtained in moruloids. The results show that no compound exceeded the effect of y27632 (Figure 5).

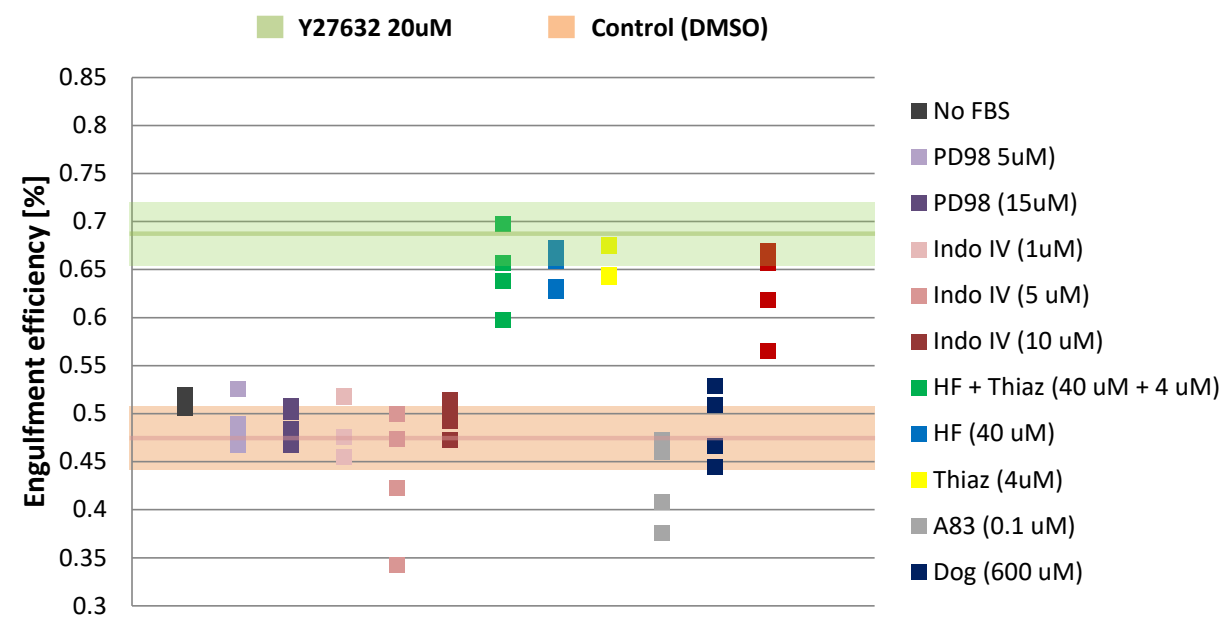

Figure 5: A panel of small molecules tested for their effect on the engulfment efficiency in moruloids. No compounds showed a larger effect than the positive control y27632. N=4 for all compounds: PD98 is the MAPK/ERK inhibitor PD98059, Indo $V$ is the PKC activator Indolactam $V, H F$ is the ROCK inhibitor Hydroxyfasudil, Thiaz is the ROCK inhibitor Thiazovivin. A83 is the TGF $\beta 1$ receptor inhibitor A-83-01 and Dog is the PKC activating DAG analog Dioctanoyl. Bands for positive (y27632) and negative (DMSO) control demarcate +1 and -1 SD from average value, $\mathrm{N}=16$ wells.

\section{Screening the Prestwick chemical library}

To further increase the moruloids engulfment efficiency on top of the positive control y27632 the Prestwick chemical library was screened (Figure 6A and B). The Prestwick chemical library is a collection of 1280 small molecules that are all FDA approved drugs and aliquoted in 96-wellplates at 
a $10 \mathrm{mM}$ concentration dissolved in DMSO. For screening, the compounds were administered in a working concentration of $20 \mu \mathrm{M}$ distributed over 15 plates including negative controls (no y27632) and basic controls (with y27632), and incubated for 24 hours with TS cells allowing them to engulf the ES bodies (Figure 6B).

\section{Trichlorfon and Ethacrynic acid}

Eight hits were selected that scored between 2 and 5 standard deviations higher than the y27632 basic control: Trichlorfon, Indapamide, Ampyrone, Midodrine, Fentiazac, Etretinate, Oxyphenbutazone and Ethacrynic acid. These hits were then validated using a dose response from 0.2 to $100 \mu \mathrm{M}$, which indicated two compounds as true hits. One is Ethacrynic acid, which is known for exerting a glutathione S-transferase inhibiting effect, and boosted the average engulfment efficiency by $13 \%$ at an optimal concentration of $20 \mu \mathrm{M}$. Although $100 \mu \mathrm{M}$ of Ethacrynic acid led to the highest engulfment efficiency, cell adherence was impaired and individual cells could be distinguished, thereby prohibiting the formation of a polarized monolayer (Figure 6C). Trichlorfon, an anthelminthic prodrug that exerts a cholinesterase inhibiting effect, was the second validated hit and boosted the engulfment efficiency by $14 \%$ at a concentration of $20 \mu \mathrm{M}$ (Figure $5 \mathrm{C}$ ). When both Trichlorfon and Ethacrynic acid were administered together, the engulfment efficiency reached a $18 \%$ higher than control, which was higher than for each of the individual compounds (Figure 5D), which suggests that both hit compounds exert an independent molecular effect.

A

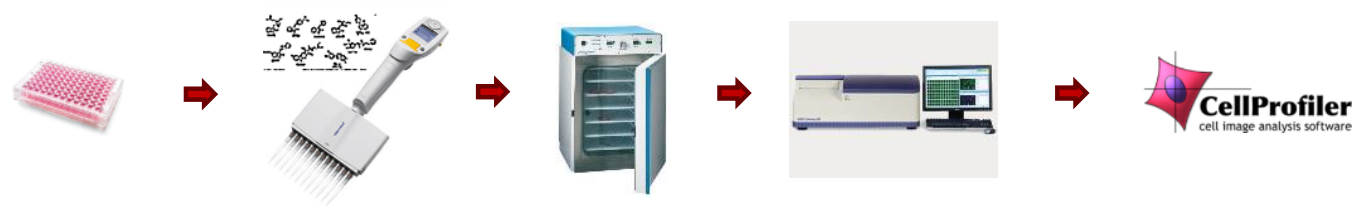

B

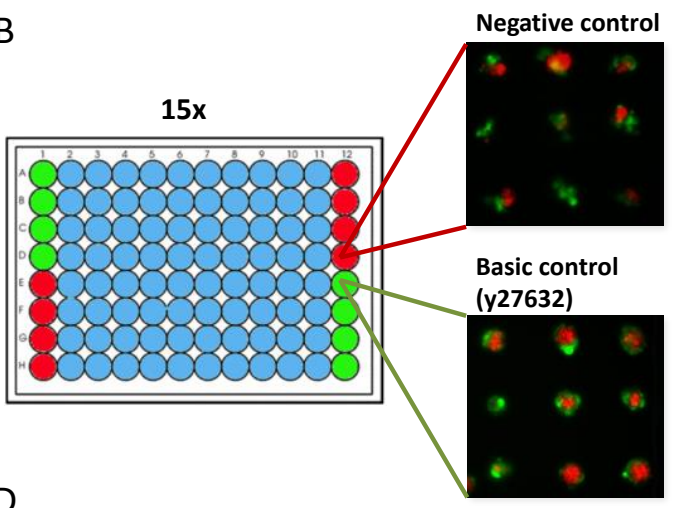

C
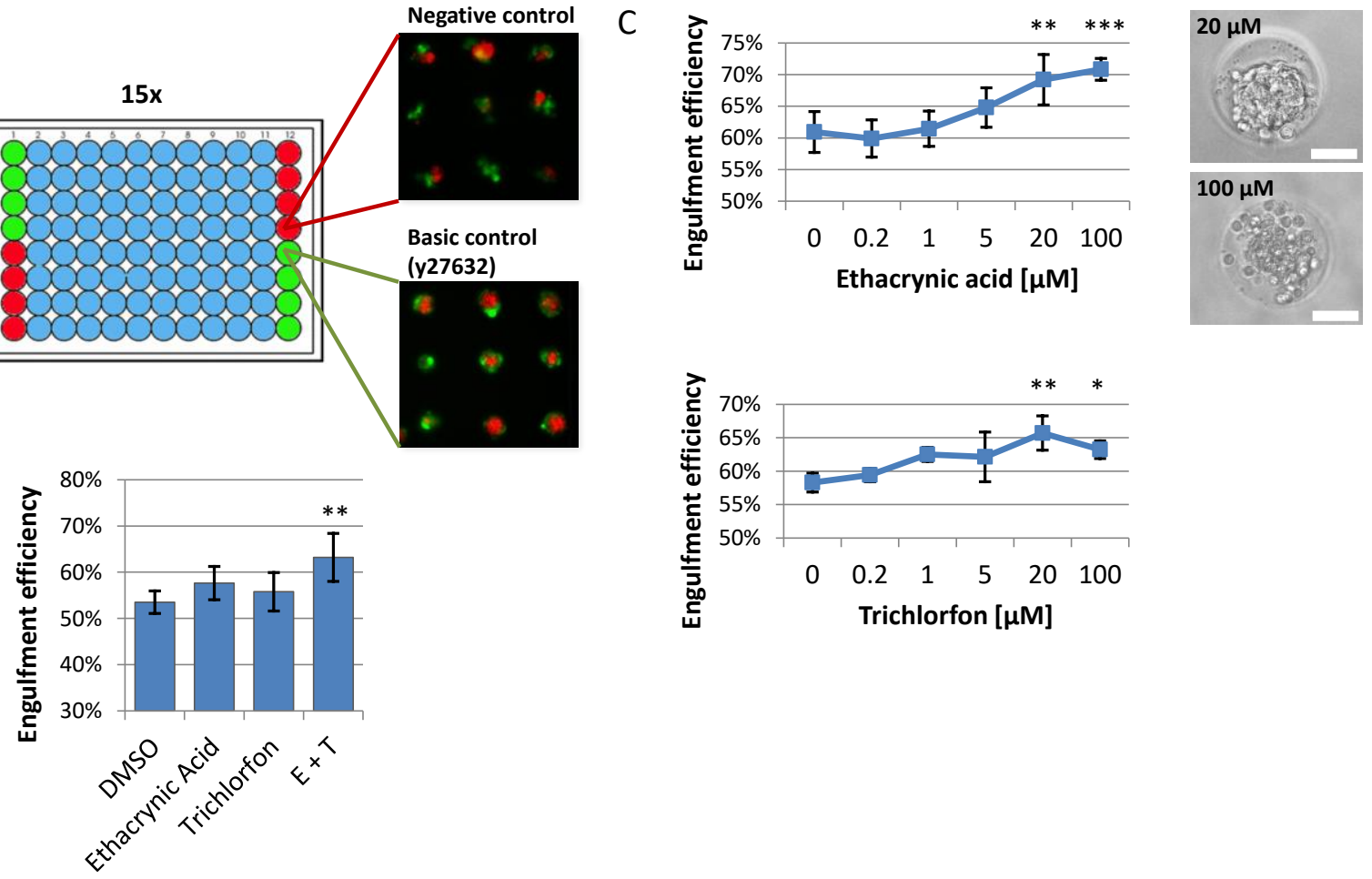

Trichlorfon $[\mu \mathrm{M}]$

Figure 6: Screening the Prestwick chemical library to find small molecules that increase the engulfment efficiency of TS cells on ES bodies. (A) Schematic overview of screening approach displaying stem cell culture 
on a hydrogel microwell screening plate, administration of the Prestwick chemical library, incubation, highcontent imaging and analysis using CellProfiler. (B) 15 hydrogel microwell screening plates were used to screen the Prestwick chemical library, each plate including a different subset of small molecules and negative (no y27632) and basic controls (with y27632). (C) Two hits from the primary screen were validated: Ethacrynic acid and Trichlorfon. A dose response curve confirmed $20 \mu \mathrm{M}$ as the optimal working concentration for both compounds. Bright field images showed the difference in cell-cell adhesion between 20 and $100 \mu \mathrm{M}$ Ethacrynic acid. Scale bar depicts $50 \mu \mathrm{m}$. (D) Combining Ethacrynic acid and Trichlorfon resulted in a higher engulfment efficiency than for the individual compounds. ${ }^{*}$ and ${ }^{* *}$ denote a statistical significance of $p<0.05$ and 0.01 respectively, ANOVA with Tukey's post-hoc test, $n=3$ for ethacrynic acid and $n=4$ for Trichlorfon, $n=16$ for controls.

\section{Discussion}

\section{Hits on engulfment efficiency}

The function of Ethacrynic acid may be an indirect one and explained by its effect of decreasing the phase II metabolism of xenobiotics, which may prolong the residence time of blastoid culture factors that promote the engulfment efficiency, such as y27632. Alternatively, Trichlorfon has an inhibiting effect on the breakdown of neurotransmitter acetylcholine. Compounds with similar characteristics may be tested for a more potent effect, such as caffeine. Altogether, the effect of hit compounds on increasing the engulfment efficiency was low compared to the large contribution that the ROCK inhibitor y27632 independently made already.

\section{Potential hits for primitive endoderm formation}

Activation of $\beta$-adrenergic receptors, leading to a rise in intracellular cAMP levels, boosted the yield of PDGFR $\alpha$-H2B-GFP expression in EBs which is indicative of PrE formation. Similar effects were obtained with exogenous CAMP in the form of 8Br-CAMP, however, the dose appeared an important factor. Of interest is the downstream activation of CAMP, either through EPAC, PKA or a combination as it is described that both pathways may act independently, converge synergistically, or oppose each other in regulating a specific cellular function ${ }^{30}$.

Notably, Bisoprolol and Betaxolol, being $\beta$-adrenoceptor blockers which prevent a downstream increase in cAMP levels, also increase the yield. These $\beta$-blockers may contribute in conditions with serum and $8 \mathrm{Br}$-cAMP in lowering an thus optimizing the effective net levels of intracellular cAMP. Similar effects result from compounds that activate the Gi subunit, such as 5-HT1R agonists BY7378 and Almotriptan. Collectively, the results strongly indicate that rising CAMP levels induce differentiation into the PrE lineage but with an optimal concentration below $1 \mathrm{mM}$. Other optimizations that can be done is the timing, frequency and duration of GPCR activation. For example, in $\beta 2$-adrenergic receptors it is described that its prolonged activation can reach a phosphorylation threshold that may result in switching the G-protein coupling of a Gs class to a Gi class ${ }^{31}$, hereby initiating a feedback mechanism by inhibiting the production of cAMP from ATP. The challenge now is to find that concentration and the temporal dynamics with it.

Moreover, it would be interesting to study the necessary context of $\beta$-adrenergic/cAMPmediated PrE differentiation. For example, others have described that EBs show an increased basal level of $\mathrm{G}_{\alpha \mathrm{s}}$-activation compared to pluripotent ES cells ${ }^{32}$, suggesting cell-cell adhesion is involved. 
Also, results from chapter 2 suggest that cell adhesion is essential in forming primitive endoderm. There, cell adhesion was disrupted during EB culture after administering the small molecules KN-62 and KN-93 (inhibitors of $\mathrm{Ca} 2+/$ calmodulin-dependent protein kinase II (CaMKII)), leading to noncompacted EBs, characterized by round distinguishable cells within the EB, and no PDGF $\alpha$-H2B-GFP activity.

\section{Methods}

\section{Cell expansion}

The expansion ES cells and TS cells was performed as described in Chapter 4 (methods section).

\section{EB culture}

EBs were formed during 24 hours in ES-medium supplemented with $10 \mathrm{ng} / \mathrm{mL}$ LIF (Invitrogen) and $50 \mu \mathrm{M} \beta$-Mercaptoethanol (Sigma). Medium was then replaced by ES-medium without Lif for standard EB differentiation culture or by basic blastoid culture medium.

B27N2 medium consisted of a 1:1 ratio of DMEM/F12 (Life Technologies) and Neurobasal medium (Life Technologies) supplemented with 1\% N2 and 2\% B27 (Life Technologies), $1 \mathrm{mM}$ Glutamax (Life Technologies), 1\% Non-Essential Amin Acids 100x (NEAA, Life Technologies), 100U/mL penicillin (Life Technologies), $100 \mathrm{mg} / \mathrm{mL}$ streptomycin (Life Technologies), $0.5 \%$ bovine serum albumin (Sigma), 10mM HEPES (Fisher Scientific), 1mM Sodium Pyruvate (Life Technologies) freshly supplemented with $0.05 \mathrm{mM}$ 2-mercaptoethanol (Life technologies).

Blastoid culture medium comprised of B27N2 medium supplemented with $10 \mathrm{ng} / \mathrm{mL}$ leukemia inhibitory factor (LIF, Life Technologies), 10\% fetal bovine serum (selected batch), $10 \mu \mathrm{M}$ CHIR99021 (GSK3 $\beta$ inhibitor, Axon Medchem), $20 \mu \mathrm{M}$ y27632 (Enzo Life Sciences), 1mM 8Br-cAMP (Biolog).

\section{Screening EBs with a GPCR ligand library}

The screen was performed in two different conditions of media: 1) B27N2 medium, and 2) b27N2 medium supplemented with $10 \%$ fetal bovine serum and the blastoid factors $10 \mu \mathrm{M} \mathrm{CHiR}$, $1 \mathrm{mM} 8 \mathrm{Br}$-cAMP and $20 \mu \mathrm{M}$ y27632. The standard procedure for both screens included seeding $13 \mathrm{ES}$ cells per microwell followed by a time window of $24 \mathrm{~h}$ in ES-medium supplemented with LIF to allow EB formation. At 0 and 48 hours, medium was replaced by the appropriate medium and the GPCR compounds (dissolved in DMSO at a concentration of $10 \mathrm{mM}$ ) were administered at a working concentration of $20 \mu \mathrm{M}$. After 96 hours of culture the EBs were washed $3 x$ using PBS and fixated with PFA $4 \% \mathrm{w} / \mathrm{v}$ overnight at $4^{\circ}$ degree $\mathrm{C}$. The day after, cell nuclei of EBs were fluorescently stained using DAPI for 1 hour. Wells were washed 3x using PBS and approximately 430 EBs per well were imaged using a 10x objective and montage function on the BD Pathway 435 system. A custom Matlab script was used to reduce background fluorescence. Then using CellProfiler ${ }^{33}$, the projection area of EBs was measured as a proxy for size and they were classified into structures positive for PDGFRa-H2B-GFP based on intensity thresholding. 


\section{Screening for improved engulfment efficiency}

An average of 10 ES cells per microwell was seeded onto the microwell arrays of the hydrogel microwell screening plate in ES medium supplemented with $10 \mathrm{ng} / \mathrm{mL}$ LIF (Invitrogen) and $50 \mu \mathrm{M} \beta$ Mercaptoethanol (Sigma). ES cells were allowed to aggregate for 24 hours before ES medium was removed and an average of 17 TS cells was dispensed on top in B27N2 medium containing $10 \%$ fetal bovine serum (selected batch), 10ng/mL LIF, $10 \mu \mathrm{M}$ CHiR, $20 \mu \mathrm{M}$ y27632 and $1 \mathrm{mM} 8 \mathrm{Br}$-cAMP. To discriminate between ES and TS cells an ES cell line comprising a constitutively active H2B-RFP fluorescent reporter was used in combination with TS cells that were live stained using Celltracker green CMFDA. Hydrogel microwells were washed 3x using PBS before approximately 180 moruloids per well were imaged using a 10x objective and montage function on the BD Pathway 435 system. Analysis was done as described in chapter 4.

\section{References}

1. Wagner, B.K. \& Schreiber, S.L. The Power of Sophisticated Phenotypic Screening and Modern Mechanism-of-Action Methods. Cell chemical biology 23, 3-9 (2016).

2. Eggert, U.S. The why and how of phenotypic small-molecule screens. Nature chemical biology 9, 206209 (2013).

3. Moffat, J.G., Rudolph, J. \& Bailey, D. Phenotypic screening in cancer drug discovery - past, present and future. Nature reviews. Drug discovery 13, 588-602 (2014).

4. Zanella, F., Lorens, J.B. \& Link, W. High content screening: seeing is believing. Trends in biotechnology 28, 237-245 (2010).

5. Zhu, Z. \& Huangfu, D. Human pluripotent stem cells: an emerging model in developmental biology. Development 140, 705-717 (2013).

6. Tape, C.J. Systems Biology Analysis of Heterocellular Signaling. Trends in biotechnology (2016).

7. Ohnishi, Y. et al. Cell-to-cell expression variability followed by signal reinforcement progressively segregates early mouse lineages. Nature cell biology 16, 27-37 (2014).

8. Hermitte, S. \& Chazaud, C. Primitive endoderm differentiation: from specification to epithelium formation. Philosophical transactions of the Royal Society of London. Series B, Biological sciences 369 (2014).

9. Wamaitha, S.E. et al. Gata6 potently initiates reprograming of pluripotent and differentiated cells to extraembryonic endoderm stem cells. Genes \& development 29, 1239-1255 (2015).

10. Wang, Y., Smedberg, J.L., Cai, K.Q., Capo-Chichi, D.C. \& Xu, X.X. Ectopic expression of GATA6 bypasses requirement for Grb2 in primitive endoderm formation. Developmental dynamics : an official publication of the American Association of Anatomists 240, 566-576 (2011).

11. Hoch, R.V. \& Soriano, P. Roles of PDGF in animal development. Development 130, 4769-4784 (2003).

12. Artus, J., Panthier, J.J. \& Hadjantonakis, A.K. A role for PDGF signaling in expansion of the extraembryonic endoderm lineage of the mouse blastocyst. Development 137, 3361-3372 (2010).

13. Cai, K.Q., Capo-Chichi, C.D., Rula, M.E., Yang, D.H. \& Xu, X.X. Dynamic GATA6 expression in primitive endoderm formation and maturation in early mouse embryogenesis. Developmental dynamics : an official publication of the American Association of Anatomists 237, 2820-2829 (2008).

14. Paca, A. et al. BMP signaling induces visceral endoderm differentiation of XEN cells and parietal endoderm. Developmental biology 361, 90-102 (2012).

15. Doughton, G., Wei, J., Tapon, N., Welham, M.J. \& Chalmers, A.D. Formation of a polarised primitive endoderm layer in embryoid bodies requires fgfr/erk signalling. PloS one 9, e95434 (2014).

16. Moore, R., Cai, K.Q., Tao, W., Smith, E.R. \& Xu, X.X. Differential requirement for Dab2 in the development of embryonic and extra-embryonic tissues. BMC developmental biology 13, 39 (2013).

17. Simandi, Z., Balint, B.L., Poliska, S., Ruhl, R. \& Nagy, L. Activation of retinoic acid receptor signaling coordinates lineage commitment of spontaneously differentiating mouse embryonic stem cells in embryoid bodies. FEBS letters 584, 3123-3130 (2010). 
18. Cho, L.T. et al. Conversion from mouse embryonic to extra-embryonic endoderm stem cells reveals distinct differentiation capacities of pluripotent stem cell states. Development 139, 2866-2877 (2012).

19. Stavridis, M.P., Collins, B.J. \& Storey, K.G. Retinoic acid orchestrates fibroblast growth factor signalling to drive embryonic stem cell differentiation. Development 137, 881-890 (2010).

20. Capo-Chichi, C.D. et al. Perception of differentiation cues by GATA factors in primitive endoderm lineage determination of mouse embryonic stem cells. Developmental biology 286, 574-586 (2005).

21. Plusa, B., Piliszek, A., Frankenberg, S., Artus, J. \& Hadjantonakis, A.K. Distinct sequential cell behaviours direct primitive endoderm formation in the mouse blastocyst. Development 135, 30813091 (2008).

22. Vassilatis, D.K. et al. The G protein-coupled receptor repertoires of human and mouse. Proceedings of the National Academy of Sciences of the United States of America 100, 4903-4908 (2003).

23. Howard, A.D. et al. Orphan G-protein-coupled receptors and natural ligand discovery. Trends in pharmacological sciences 22, 132-140 (2001).

24. Overington, J.P., Al-Lazikani, B. \& Hopkins, A.L. How many drug targets are there? Nature reviews. Drug discovery 5, 993-996 (2006).

25. Gilman, A.G. G proteins: transducers of receptor-generated signals. Annual review of biochemistry 56, 615-649 (1987).

26. Keiser, M.J. et al. Relating protein pharmacology by ligand chemistry. Nature biotechnology 25, 197206 (2007).

27. Enserink, J.M. et al. A novel Epac-specific CAMP analogue demonstrates independent regulation of Rap1 and ERK. Nature cell biology 4, 901-906 (2002).

28. Leech, C.A. et al. Facilitation of ss-cell K(ATP) channel sulfonylurea sensitivity by a cAMP analog selective for the cAMP-regulated guanine nucleotide exchange factor Epac. Islets 2, 72-81 (2010).

29. Fleming, T.P., Sheth, B. \& Fesenko, I. Cell adhesion in the preimplantation mammalian embryo and its role in trophectoderm differentiation and blastocyst morphogenesis. Frontiers in bioscience : a journal and virtual library 6, D1000-1007 (2001).

30. Cheng, X., Ji, Z., Tsalkova, T. \& Mei, F. Epac and PKA: a tale of two intracellular cAMP receptors. Acta biochimica et biophysica Sinica 40, 651-662 (2008).

31. Chen-Izu, Y. et al. G(i)-dependent localization of beta(2)-adrenergic receptor signaling to L-type $\mathrm{Ca}(2+)$ channels. Biophys J 79, 2547-2556 (2000).

32. Layden, B.T., Newman, M., Chen, F., Fisher, A. \& Lowe, W.L., Jr. G protein coupled receptors in embryonic stem cells: a role for Gs-alpha signaling. PloS one 5, e9105 (2010).

33. Carpenter, A.E. et al. CellProfiler: image analysis software for identifying and quantifying cell phenotypes. Genome biology 7, R100 (2006). 


\section{Supplementary information}
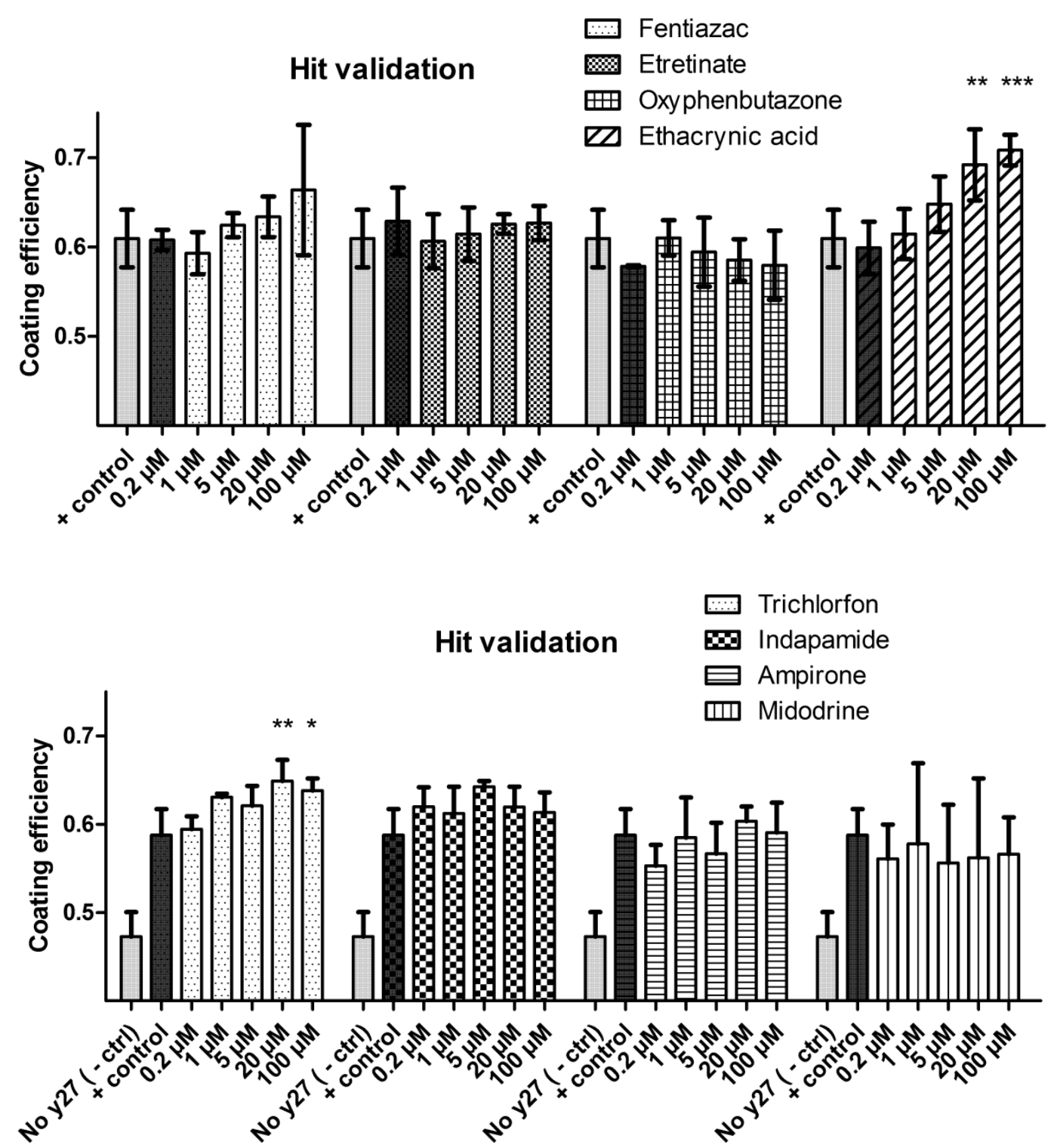

Figure S1: Eight hit compounds from the small molecule screen using the Prestwick chemical library for increasing the engulfment efficiency. The two compounds Ethacrynic acid and Trichlorfon were validated as hits at a working concentration of $20 \mu \mathrm{M}$. * and ** denote a significance of $p<0.05$ and 0.01 respectively, ANOVA with Tukey's post-hoc test, $n=3$ for top graphs and $n=4$ for bottom graphs, for controls $n=16$. 
Chapter 5 
Chapter 6 


\section{Multicellular organization and morphogenesis}

As was described in chapter 2, cellular building blocks are naturally fusing without an external applied force (self-assembly). Such tissue deformation and remodeling occurs by internal, cellgenerated forces, which are fundamental forces shaping tissues and organ development. Nuclear and actin staining showed that cells are still properly distributed and form a continuous tissue after tissue shaping processes have taken place.

\section{Self-organization}

Recent advances in omics (e.g. genomic and proteomic) technologies combined with big data approaches such as machine- and deep-learning shape a plausible near future where humanity obtains a detailed picture of the genetic and molecular regulatory mechanisms involved in tissue and organ formation. However, similar to brain simulation approaches where every synapse is mapped ${ }^{1}$ is unlikely to result in an understanding of consciousness, a complete map of all expressed genes does not explain the formation of an organ or organism. Yet, important clues are elucidated that allow the interrogation of tissue and organ formation, which can be exploited to recapitulate them in vitro using the self-organization capacity they possess.

These self-organizational processes are believed to be based on adaptive feedback control systems. Methods based on the same mathematical principles used in system engineering, such as mechanical system dynamics, electrical engineering, were already engaged by Turing to describe the diffusion kinetics responsible for tissue formation ${ }^{2}$. Genetic engineering is using these same principles to map, model and predict the behavior of genes within the cell in time and space.

\section{Can we push engineered tissues to the next level?}

The elegant aspect of self-organization in biology is that it functions at different scales of size. When a minimal level of understanding is attained about the mechanisms coordinating cellular action, one can use these as semi-autonomous building blocks in forming a structure of higher order and function. As a matter of fact, the possibilities of recapitulating tissue and organ development in vitro are rapidly branching out. It is astonishing to see the extent to which tissues can develop from the right combination of cells in vitro with none or a limited set of external signals, thus in principle developing in isolation from their native tissue surroundings.

So far, many of these in vitro generated organs (i.e. organoids) are either small-scale models or they replicate a repeating organ or tissue module comprised of a multicellular structure with a specific tissue or organ function, such as lobules from the liver and breast, islets of Langerhans, lymph nodes or intestinal and colonic crypts. By spatially ordering these multicellular functional units, for example by directed self-assembly, a tissue of higher biomimicry and complexity can be created.

Mechanical and biochemical cues, including morphogen gradients, synergistically regulate tissue morphogenesis and organogenesis, including their integration and macro-shaping. For example, chimeric mice deficient for a critical transcription factor for pancreas development were injected with wild-type pluripotent stem cells from a rat and developed a full pancreas with the shape and size of that from a mouse ${ }^{3}$. This demonstrates that the macro-features of organ complementation 
occurs on terms of the host species and is thus directed by signals from the surrounding tissues, indicative of a developmental niche including physical signals.

Several crucial aspects of developing tissues are still challenging to implement in vitro, such as innervation and blood vessels. In organoid models the lack of such structures often hinders their maturation. Another essential contributor is the proper distribution of physical forces, arising from fluid pressure, ECM reinforcements or cell buckling for instance. These forces may well be recapitulated using bioreactors. Mathematical frameworks derived from studies on soft biomechanics for example, can help to describe the force vectors necessary for growth and remodeling of tissues ${ }^{4}$.

Importantly, strategies using directed assembly of tissue modules and organoids may elucidate how stem cell niches from multiple tissue types or organs interact and reciprocally coordinate their structure and cellular composition during integration and remodeling. This particularly applies to the interaction with stroma which supports the function of surrounding tissues and organs, physically and biochemically. By combining strategies such as micro-patterning, directed self-assembly and directed differentiation, by using simple and practical microfabricated tools, tissues and organs can be made physiologically and functionally more similar to their native analogues. In addition, modulating circuits of molecular signaling by either genetically editing specific cell types within a developing tissue or by using high-throughput screening of soluble factors, may shine light on the parameters involved in complex tissue formation. Advanced 3D high-content imaging, using technologies such as light sheet microscopy, would provide an attractive solution to read out these complex tissues.

\section{The blastoid}

\section{Plasticity and redundancy}

Self-organization, which can be defined as the formation of structures with complex patterns built by local interactions between units of less complexity, is heavily exploited in biological systems

${ }^{2}$. It has been proposed that all the interdepend factors that affect developmental processes such as cell decision making, symmetry breaking and lineage divergence can be reconciled if early embryonic development is considered as a self-organizing system ${ }^{5}$. Internal interactions form feedback loops, thereby establishing a degree of robustness to these systems.

Although it is known that the activation of the zygotic genome is tightly regulated to transiently take over the maternal instructions, provided as mRNA transcript, maternal factors are not known to be essential for patterning of the mammalian embryo. These findings are congruent with computational modeling of preimplantation development, which underlines the absence of an a priori need for intrinsic differences in blastomeres ${ }^{6}$. Essentially, embryo patterning processes, including the extensively studied symmetry breaking of blastomeres, occur by self-organization. Herein, apparent stochastic processes are reinforced and supported by feedback loops ${ }^{5}$. 
Signaling redundancy contributes to robustness. For example, a molecular signaling process that conveys genetic redundancy is the fate decision between TE and ICM cells, in which Tead4 and Notch signaling play a redundant role in the polarity-dependent HIPPO-mediated regulation of their fate-specific gene expression ${ }^{7}$. Another example is trophectoderm fate allocation for which the amount of cell-cell adhesion is a determinant ${ }^{8}$. The abundance of cell-cell contact is mediated by the intensity of extracellular cadherin domains but also by the position and number of cells surrounding it. This is highlighted by studies that showed when dissociated cells from a 32-cell stage embryo are randomly re-aggregated, TE and ICM precursors spatially re-organize themselves and blastocysts form that develop into normal mice ${ }^{9}$.

Surprisingly, ES and TS cells did not spontaneously rearrange their position into a pluripotent core with a TS peripheral monolayer. Instead, they segregated and formed two attached but distinct cell populations. Therefore, in moruloids the embryonic compartment (i.e. EB) was pre-formed and the seeding number of TS cells and their actin organization was adjusted (using a ROCK inhibitor) to facilitate effective engulfment into a polarized TE monolayer. This spatial organization might reinforce trophectoderm-specific gene expression, similar as in the developing embryo. Clearly, the formation of a blastoid demonstrates that cellular and molecular underpinnings of morphogenetic events such as epithelialization of the TE and cavitation are obviously not pre-patterned in the egg or at any stage prior to morula formation and cell fate specification.

\section{Implementing microfluidics into microwell systems}

Exposing cellular bodies to bulk culture conditions permits merely a crude approximation of the intricate combination of stimuli found during embryogenesis. In order to faithfully recapitulate the signaling during embryogenesis, such as the soluble cues between the uterus and the periimplantation embryo, one needs an in vitro culture system that allows administering soluble factors in a tightly controlled spatiotemporal fashion. The designated solution can probably be found in microfluidics. Hence, these systems should be adapted to prove compatibility with 3D cultures, on which efforts are being made ${ }^{10,11}$. If such a system proves functional, it has the added benefit of minimizing the use of expensive reagents, such as growth factors, by applying micro- or nano-liter fluid handling.

\section{Ethical concerns}

\section{Potentiality of embryos}

No blastoid has yet implanted into a mouse uterus and developed to term. However, selecting blastoids that exhibit markers of implantation competence, such as a correctly aligned primitive endoderm, may increase the chances of implantation and even post-implantation embryonic development. It is important to emphasize that this applies to mouse, and therefore largely circumvents the scrutiny that would apply when equaling this research to human material.

Yet, if it would be possible to translate these results in mice to human, either by using extraembryonic tissues differentiated from pluripotent stem cells or from animal origin, this is expected to provoke controversy which requires to inspect the ethical and legal ramifications. As of now, the Human Fertilization and Embryology Act (HFEA) that was signed in 1990 permits human 
embryo experimentation up till 14-days or till the formation of a primitive streak ${ }^{12}$. However, voices are rising to revisit this rule in the light of new technologies and its potential benefits ${ }^{13}$.

An embryo has the potential to become a full grown organism, for instance a mouse or a human. The route of embryo development from conception to birth is the development of an organism, with a gradual increase in complexity. It is questionable whether a specific breaking point can be specified when an embryo (i.e. fetus) becomes an organism (i.e. human). One definition holds that an organism should be able to maintain its vital processes. Arguments about abortion add to this definition the possibilities of using external instruments in attaining this goal. In case of experimentation, the aforementioned point that HFEA agreed on was chosen based on the last point at which twinning can occur.

A preimplantation stage embryo is far from both these points in development. Explicitly, a preimplantation embryo has merely formed the extraembryonic compartments necessary to connect to the host and further sustain development of the embryo. These extraembryonic tissues are discarded at birth and therefore regarded as a temporary necessity, not required for mature function. This implicates that the 'uniform' pluripotent epiblast cell mass of the peri-implantation embryo is at a similar stage of mature organismal development as compared to a fertilized egg cell.

Viewing development as a process of gradually acquiring the state of individuality holds that an embryo has higher moral value than cells due to its autonomous potential. This also discriminates an embryo from pluripotent stem cells that are capable of forming an entire organism, as the latter need support (i.e. external manipulation) to exploit their similar level of potential.

\section{Clonal origin of blastoids}

As blastoids are derived from a single pluripotent cell line, and thus share identical genetic code, they are considered clonal. Speculating one step further, when blastoids would be able to be reproduced using human cells, such technology can be used to form a multitude of human embryos sharing an identical genetic make-up. This would violate the laws that prohibited human cloning many countries. Yet, other countries or states have no legislation on this topic or only permit therapeutic cloning ${ }^{14}$, which may not, however, per se be interpreted as a tolerance position towards human cloning when the technological possibilities present themselves. 


\section{References}

1. Union, E. human brain project framework partnership agreement. www.humanbrainproject.eu (2015).

2. Turing, A.M. The chemical basis of morphogenesis. 1953. Bulletin of mathematical biology 52, 153197; discussion 119-152 (1990).

3. Kobayashi, T. et al. Generation of rat pancreas in mouse by interspecific blastocyst injection of pluripotent stem cells. Cell 142, 787-799 (2010).

4. Gjorevski, N. \& Nelson, C.M. The mechanics of development: Models and methods for tissue morphogenesis. Birth defects research. Part C, Embryo today : reviews 90, 193-202 (2010).

5. Wennekamp, S., Mesecke, S., Nedelec, F. \& Hiiragi, T. A self-organization framework for symmetry breaking in the mammalian embryo. Nature reviews. Molecular cell biology 14, 452-459 (2013).

6. Honda, H., Motosugi, N., Nagai, T., Tanemura, M. \& Hiiragi, T. Computer simulation of emerging asymmetry in the mouse blastocyst. Development 135, 1407-1414 (2008).

7. Frum, T. \& Ralston, A. Cell signaling and transcription factors regulating cell fate during formation of the mouse blastocyst. Trends in genetics : TIG 31, 402-410 (2015).

8. Nishioka, N. et al. The Hippo signaling pathway components Lats and Yap pattern Tead4 activity to distinguish mouse trophectoderm from inner cell mass. Developmental cell 16, 398-410 (2009).

9. Suwinska, A., Czolowska, R., Ozdzenski, W. \& Tarkowski, A.K. Blastomeres of the mouse embryo lose totipotency after the fifth cleavage division: expression of Cdx2 and Oct4 and developmental potential of inner and outer blastomeres of 16- and 32-cell embryos. Developmental biology 322, 133144 (2008).

10. Cosson, S. \& Lutolf, M.P. Hydrogel microfluidics for the patterning of pluripotent stem cells. Scientific reports 4, 4462 (2014).

11. Occhetta, P. et al. High-Throughput Microfluidic Platform for 3D Cultures of Mesenchymal Stem Cells, Towards Engineering Developmental Processes. Scientific reports 5, 10288 (2015).

12. Pera, M.F. et al. What if stem cells turn into embryos in a dish? Nature methods 12, 917-919 (2015).

13. Hyun, I., Wilkerson, A. \& Johnston, J. Embryology policy: Revisit the 14-day rule. Nature 533, 169-171 (2016).

14. Human cloning laws. National conference of state legislatures (2008). 
Chapter 6 


\section{Chapter 7}

Valorization of Research Findings 
Chapter 7 


\section{Valorization}

The term "valorization" includes the use or application of an invention, object, process or activity so that it generates value. In the European Union's perspective on academic research, this can be described by the dissemination and exploitation of the results obtained within a given project. Here, a few potentially interesting options are outlined for valorizing the research described in this thesis.

\section{Microwell platforms}

Two microwell screening platforms are described in this thesis, namely 1) the (wet) agarose hydrogel-based microwell screening platform which is incorporated into a standard transparent polystyrene 96-well culture plate, and 2) the thermoformed microwell platform which is based on a (dry) thermoformed COP film that is bonded to a bottomless standard 96-well plate. Both share a similar purpose but have their own characteristics. Above all, the hydrogel system is wet and thus requires additional conduct to preserve sterility. The benefit of a wet system is the absence of noticeable diffraction differences between the microwell material and the culture medium. Conversely, the advantages of using the thermoformed system are its accessible handling and compatibility with most (confocal) microscopy objectives, as the sample-objective distance is smaller.

The powerful aspect of microwell screening arrays is their versatility in assay application. First of all, many cell types allow their culture as unanchored aggregates, either as single cell type spheroids or multicellular aggregates that can organize and develop into structures that replicate tissues and organs, including diseased ones. Moreover, microwells can be modified to allow cells attaching onto a surface or to anchor cells within a substrate of ECM components. As of the hydrogel microwell screening platform, initial trials were performed of modifying the hydrogel material, in this case gelatin, as it is cheap and easy-to-handle. However, the system can expectedly be tailored with a range of hydrogel materials such as fibronectin, collagen or matrigel that could extent culture options to tissues and organoids necessitating their integration into such ECM-like microenvironments ${ }^{1}$. Similarly, thermoformed microwell plates permit the engraftment of ECM components such as a basal membrane-like substrates onto the polymer film. As such, functionalized surfaces can be introduced as a (patterned) interface to control cell adhesion, morphology and differentiation.

\section{The competition}

Recently, various companies have been founded that jump in on the trend of 3D cultures and their analysis. The company Microtissues sells the '3D Petri Dish' which includes a polymeric stamp used for replica molding microwells into hydrogel, however not directly in multiwell plates but on a tissue culture surface such as a standard single dish. Alternatively, the company Stemcell licensed the product 'AggreWell' ${ }^{\mathrm{TM}}$ ' which is based on standard multiwell plates, specifically 6-well plates. They offer a large array of polymeric microwells integrated into the multiwell dish. Similarly, the enterprise Elplasia offers a range of standard multiwell plate-based microwell platforms comprised of either square or round bottom microwells. As a 96-wellplate is also in their assortment, this 
permits high-throughput screening (HTS) of aggregates with a decent biological replicate number per well/ condition.

Others also attempt to translate cell aggregate cultures to the realm om HTS. The company 3DBiomatrix sells the 'Perfecta3D ${ }^{\circledR}$ Hanging Drop Plate' in a 96 and 384 format that allows aggregation by the classical hanging drop methods and accessible imaging, although only one cellular structure is formed per "well". An identical approach is pursued by the company InSphero with their 'GravityTRAP'M ULA Plate'.

Then there is Alvetex, that does not focus on self-organizing tissues but offers cells a polymeric scaffold substrate in the form of inserts for existing standardized culture platforms. Others do not sell the platform but instead provide services for 3D high-content imaging and analysis of over 150 different 3D cellular models. The company Ocello generates these models in-house and employs them for phenotypic screening and compound profiling. As an extra service they perform in-depth 3D imaging and analysis on plates from customers.

\section{Unique selling point}

The valorization potential of the microwell screening platforms - as they are now - regards the massively parallelized culture of non-adherent and matrix-free 3D cellular aggregates. The unique selling point of both the thermoformed and hydrogel-based platform is this integration within a 96wellplate format which renders their compatibility with HTS. Importantly, every well contains more than hundred microwells which provides statistical robustness in screening assays. Also, both platforms are compatible with liquid handling machines and high-content imaging systems. Comparing with competitor's platforms, the round-bottom plates from Elplasia display the highest similarity to our thermoformed and hydrogel microwell plates. However, the imaging possibilities of this plate are so far unknown as the information provided by Elplasia is limited.

\section{The hydrogel microwell platform}

The hydrogel microwell platform is already successfully applied in a variety of research projects. Examples include the controlled re-aggregation of primary human pancreatic islet cells ${ }^{2}$ and the facilitation of changing identity of mature human beta-cells into glucagon-producing alpha-cells ${ }^{3}$, which have potential impact on regenerative strategies in diabetes.

The polymeric mold is the most valuable part of this microwell replica-molding system. It contains pillars placed in an arrayed format for 96-well plates. Every pillar contains hundreds of micropillars that are copied into an agarose hydrogel by replica molding which is a sort of stamping. Two potential modifications to the system that may be considered for improvement:

- Speeding up the production; the stamp material may be substituted to something that is more stiff and easily releases from the agarose hydrogel without the need of a wet environment. For example, a thermoplastic polymer may be considered, such as polystyrene. However, polystyrene has a linear expansion coefficient of $0.7 \%$ per 100 degree, yielding in shrinkage. This in contrast to $0.03 \%$ for silicon. Alternatively, an antisticking coating may be applied such that the stamp can be released more easily after molding the hydrogel. 
- The microwell geometry could be modified to one with a hemispherical or conical shaped bottom instead of a flat one. This would extend the rapid formation of aggregates to cells that are resilient to spontaneous aggregation.

\section{The thermoformed microwell platform}

Meanwhile, thermoformed microwell systems have been used for a plethora of cell biological studies in academic research. For example as a promising approach in confining Individual islets, thereby providing a protective environment to preserve islets during and after transplantation ${ }^{4}$. Another study showed augmented bone formation in 3D aggregate cultures from human osteoblasts under fluid flow-mediated biomechanical stimulation ${ }^{5}$. Such studies validate the thermoformed microwell system as a culture platform for 3D models that mimic native tissue.

\section{Capitalization}

Pioneers of microthermoforming technology have introduced a thermoformed microwell screening plate into the market through a newly founded company named 300Microns. Moreover, the focus is on providing tailored polymer film-based 3D cell culture systems. The technology for microthermoforming polymer film-based products is protected by intellectual property that is licensed from Karlsruhe Institute of Technology, where it was developed.

\section{Scaffold-free tissues of clinically relevant size}

The study that is described in Chapter 2 - The generation of scaffold-free tissues with defined shape and up to centimeter scale - is backed up by a patented invention that relates to the modular bottom-up method that was employed for producing these 3D tissues ${ }^{6}$. In particular, the invention describes the formation of tissues by combining living cells to form supracellular aggregates using geometrical confinements, followed by combining those aggregates and applying conditions that induce self-assembly and tissue morphogenesis. Chapter 2 described the formation of tissues with defined architecture by using intermediate tissue building blocks formed by human primary cells and stem cells. This opens the door of forming shaped tissues on demand from patient-derived cells. First, these cells can be multiplied in vitro, then aggregated into tissue building blocks, optionally guided using small molecules to differentiate into the appropriate tissue type, and then formed into a tissue of clinical relevant size with a defined geometry that fits to the site of implantation in the patient.

Using this method, cartilage tissue generation could be an attainable option to explore taking to the next level, for instance animal studies. Especially, recent developments in culture of articular-like cartilage from human primary chondrocytes or MSCs may contribute to this purpose ${ }^{7,8}$ and provide more authentic cartilage tissue that is adjusted in shape to fit into defect.

\section{The blastoid model}

Potential applications for the blastoid are modeling and studying embryonic development and basic biology, toxicology screenings and cloning. 


\section{Cloning}

We envision that cherry picking the blastoids that show correctly aligned primitive endoderm, covering the ICM, could be an effective strategy to increase the chances of implantation. Obviously, this necessitates an non-invasive method to spatially resolve the formation of PrE. A fluorescent reporter for PrE can provide a solution, however, challenges await in forming blastoids using wildtype cells or cells which are custom gene-edited. A potential solution could be harnessing machine learning to find a relation between phenotypical data, obtained via bright-field microscopy, and correct specification of PrE.

\section{Cloning livestock}

In case blastoids show the competence of implanting and developing to term, cloning will be a very interesting and thought-provoking application. Particular impact would be in livestock cloning, preferably cattle ${ }^{9}$. For instance, the bovine breeding community showed increased interest for in vitro assisted development of bovine embryos (in vitro embryo production - IVP) in the last decades. Nowadays many bovine embryos are produced by in vitro fertilization using sex tested semen to secure sex and high genetic value. The significant next step would be reproductive cloning of adult cattle by nuclear transfer, which is technically feasible but expensive and inefficient. As such, switching to commercially interesting bovine blastoid development should be the priority.

\section{Breeding genetically modified mice}

Gene targeting has led to hundreds of different mouse models for human disorders and these genetically modified mice are used in $2 / 3$ of all mice experiments done in the UK (Office 2014). Inactivating genes Knockout mice have revealed the roles of many genes important in embryonic development, healthy physiology and disease.

Generating knockout mice typically starts with targeting genes in ES cells which are then selected, proliferated and injected into mouse blastocysts where they mix with cells from the autologous ICM. The injected blastocysts first have to be implanted in a pseudo-pregnant mouse to grow to a full pup that hopefully contains the altered gene in its gametes. If so, Mendel's law applies to the mice that are bred allowing their altered genes to be inherited to offspring that contains this modification in all of their cells.

When blastoids are able to develop into full-grown mice, genetic modification can be easily introduced in these animals by targeting the embryonic stem cells that grow them. This would significantly speed up the process as it assures the genetic inheritance of modified genes and circumvents Mendel's law. This enables introducing multiple modifications at once to facilitate revealing functional redundancy between several genes that can mask gene function in specific knockdowns. Additionally, genetically identical mice are generated in contrast to conventional breeding methods. This would also include an advantage compared to novel in vitro methods of recapitulating complete meiosis from ES cell-derived germ cells leading to functional sperm-like cells that can lead to fertile offspring 10. 


\section{Toxicology and drug toxicity screenings}

The blastoid can be generated in a massively parallel fashion which allows screening of drugs and (potential) hazardous or toxic compounds on a genetically identical model for preimplantation development. This can have important implications on the safety regulations for prescribing drugs, which are usually not tested on pregnant women or women in the process of becoming pregnant.

\section{Basic biological studies and regenerative medicine}

An in vitro model that accurately recaps preimplantation development would greatly contribute to the study of genetic, molecular and physical mechanisms governing cell fate specification and tissue and organ differentiation and morphogenesis. Presently, the state-of-the-art method for in vitro study of preimplantation development of the mouse includes flushing out embryos from the uterus or oviduct of a pregnant mouse and continue culture in chemically-defined medium. Mice are normally sacrificed in the process of harvesting embryos and natural embryos can only be obtained in relatively small numbers. Hence, a way to prevent sacrificing animals and increase to large-scale generation and screening on early embryonic development is to utilize artificial blastocysts. Clearly, the usefulness of such a model system critically depends on its resemblance to its natural equivalent. Interestingly, however, Intestinal organoids have already been used with success in identifying novel rare cell types ${ }^{11}$ that would be difficult to discover directly in vivo.

\section{References}

1. Turner, D.A., Baillie-Johnson, P. \& Martinez Arias, A. Organoids and the genetically encoded selfassembly of embryonic stem cells. BioEssays : news and reviews in molecular, cellular and developmental biology 38, 181-191 (2016).

2. Hilderink, J. et al. Controlled aggregation of primary human pancreatic islet cells leads to glucoseresponsive pseudoislets comparable to native islets. Journal of cellular and molecular medicine 19, 1836-1846 (2015).

3. Spijker, H.S. et al. Conversion of mature human beta-cells into glucagon-producing alpha-cells. Diabetes 62, 2471-2480 (2013).

4. Buitinga, M. et al. Microwell scaffolds for the extrahepatic transplantation of islets of Langerhans. PloS one 8, e64772 (2013).

5. Altmann, B. et al. Differences in morphogenesis of 3D cultured primary human osteoblasts under static and microfluidic growth conditions. Biomaterials 35, 3208-3219 (2014).

6. Nicolas C. Rivron, J.R., Severine Le Gac, Roman K. Truckenmüller, Clemens A. van Blitterswijk and Erik J. Vrij SELF-ASSEMBLING TISSUE MODULES. (2009).

7. Leijten, J. et al. Metabolic programming of mesenchymal stromal cells by oxygen tension directs chondrogenic cell fate. Proceedings of the National Academy of Sciences of the United States of America 111, 13954-13959 (2014).

8. Caron, M.M. et al. Hypertrophic differentiation during chondrogenic differentiation of progenitor cells is stimulated by BMP-2 but suppressed by BMP-7. Osteoarthritis and cartilage / OARS, Osteoarthritis Research Society 21, 604-613 (2013).

9. Mapletoft, R.J. \& Hasler, J.F. Assisted reproductive technologies in cattle: a review. Revue scientifique et technique 24, 393-403 (2005).

10. Zhou, Q. et al. Complete Meiosis from Embryonic Stem Cell-Derived Germ Cells In Vitro. Cell stem cell 18, 330-340 (2016).

11. Grun, D. et al. Single-cell messenger RNA sequencing reveals rare intestinal cell types. Nature 525, 251-255 (2015). 
Chapter 7 


\section{Summary}

A long-sought goal in the field of regenerative medicine is to generate complex tissues as use for medical therapies (replacing damaged tissues), disease modelling and drug screening (replacing animal experiments). Classical top-down tissue engineering often involves the use of biomaterialbased scaffolds that act as a matrix template offering guidance and structural support for the cells and the tissues that are formed. Although such approaches bring freedom of geometrical design, the biological complexity of in vitro-generated tissues is often limited. More recently, bottom-up tissue engineering has emerged as a methodology to increase the complexity of tissues by exploiting selfassembly and directed development of cellular modules. Chapter $\mathbf{1}$ describes the recent technological and biological advancements that push the field of regenerative medicine towards exploiting methods of tissue-authentic and organotypic culture and how to direct and image these cultures in high-throughput. Chapter 2 then demonstrates an accessible and scalable bottom-up method to build complex three-dimensional tissues up to the centimeter scale using only cells as a building material. Here, the sequential self-assembly of cells and cell-aggregates, that are used as living, self-scaffolding building block materials, allows for the free-form fabrication of complex 3D tissues. To fine-tune the physical and biological properties of the building block, a platform was developed based on non-adherent hydrogel templates that permits high-throughput screening of small molecules and high-content imaging of phenotypical and gene-expression features.

In chapter 3 an microwell screening platform is introduced based on micro-thermoformed round-bottom microwell arrays formed from optically clear cyclic olefin polymer films. The platform is integrated in standard 96-well plates, thus facilitates the manifold formation, screening and automated imaging of organoids. The potential for high-throughput screening is validated by running a small molecule screen to direct differentiation of embryoid bodies into primitive endoderm. Using on-chip high-content imaging, we identify molecules, including modulators of the cAMP pathway, regulating tissue size, morphology and primitive endoderm-gene activity. It is speculated that platforms allowing for the reproducible, controlled and rapid formation of multiple replicates along with high-throughput screening will accelerate the use of organoids as models with greater prediction power for scientific and clinical studies.

In chapter 4 a succinct overview is given of the processes involved during preimplantation development of the mouse embryo, including the initial cell fate decisions, segregation of those cell populations and morphogenetic events. The "blastoid" is then introduced; a multicellular model formed by a combination of embryonic and trophoblast stem cells to recapitulate preimplantation development more accurately than existing models. Blastoids comprise both the embryonic and extraembryonic compartments and display similar morphology and cell allocation to blastocyst-stage embryos. High-content imaging using the hydrogel microwell screening platform allows for optimizing the formation of the blastoids using titration of cell numbers and soluble factors that modulate developmental pathways such as Wnt. In chapter 5, the Prestwick chemical library is employed to screen for compounds that increase the yield of trophoblast stem cells encapsulating embryoid bodies, in order to form blastoids. Another screen using a G-protein coupled receptor 
ligand library on embryoid bodies identified several hits, including beta-adrenergic agonists, that promote primitive endoderm formation.

In chapter 6 themes are discussed about how far we may push engineered multicellular organization in vitro and the potentiality of blastoid cultures including the related ethical concerns. Finally, in the valorization chapter $\mathbf{7}$ the potential applications are described for the microwell screening platforms, directed assembly of material-free tissues and the blastoid model. 


\section{Biography}

Born in Rotterdam, the Netherlands on November 10th, 1983, Erik was raised in the "Twentse" village Haaksbergen. In 1996 he began high school and followed the program "Natuur en Gezondheid" of "Voorbereidend Wetenschappelijk Onderwijs".

In 2002 he enrolled in the program Mechanical Engineering at the University of Twente. A year after starting a Master's study in Sustainable Energy Technology he switched to follow his passion for biology in the Biomedical Engineering Master program. In 2008 he did an internship at the 'Institute of BioEngineering of Catalonia' in Barcelona, Spain, on "in vitro study of dynamically seeding Mesenchymal Stem Cells in PLA/G5 and CaP scaffolds using a perfusion bioreactor". This work was followed by a graduation project in the Tissue Regeneration group of the MIRA institute at the University of Twente under supervision of dr. Nicolas Rivron and dr. Roman Truckenmüller. With this work on "Microfabrication of millimeter-scale geometric tissues with internal patterns of cellular compaction and capillary formation" he obtained his MSc title in 2009.

In 2010 Erik started as a PhD-student in the Tissue Regeneration group under supervision of dr. Nicolas Rivron, dr. Roman Truckenmüller and promotor prof. dr. Clemens van Blitterswijk, the results of which are contained in this book. Meanwhile in the Merln Institute at Maastricht University he is continuing work on improving primitive endoderm formation in blastoids. 


\section{Publication Record}

\section{Publications}

Pluripotent stem cells regulate trophectoderm development in vitro, Nicolas C. Rivron, Erik J. Vrij*, Javier Frias-Aldeguer*, Jean-Charles Boisset, Jeroen Korving, Roman K. Truckenmüller, Alexander van Oudenaarden, Clemens A. van Blitterswijk ${ }^{\#}$ and Niels Geijsen ${ }^{\#}$. In review at Cell

Directed Assembly and Development of Material-free Tissues with Complex Architectures. Erik Vrij, Jeroen Rouwkema, Vanessa LaPointe, Clemens Van Blitterswijk, Roman Truckenmüller and Nicolas Rivron. Advanced Materials, 2016

3D high throughput screening and profiling of embryoid bodies in thermoformed microwell plates. E. J. Vrij, S. Espinoza, M. Heilig, A. Kolew, M. Schneider, C. A. van Blitterswijk, R. K. Truckenmüller and N. C. Rivron. Lab on a Chip, 2016

Engineered Micro-Objects as Scaffolding Elements in Cellular Building Blocks for Bottom-Up Tissue Engineering Approaches. A. Leferink, D. Schipper, E. Arts, E. Vrij, N. Rivron, M. Karperien, K. Mittmann, C. Van Blitterswijk, L. Moroni and R. Truckenmüller. Advanced Materials, 2014

Tissue deformation spatially modulates VEGF signaling and angiogenesis. Nicolas C. Rivron, Erik J. Vrij, Jeroen Rouwkema, Séverine le Gac, Albert van den Berg, Roman K. Truckenmüller and Clemens A. van Blitterswijk. PNAS, 2012

Perfusion cell seeding on large porous PLA/calcium phosphate composite scaffolds in a perfusion bioreactor system under varying perfusion parameters. M. A. Koch, E. J. Vrij, E. Engel, J. A. Planell and D. Lacroix. Journal of Biomedical Materials Research Part A, 2010

\section{Features}

Shaping cells to mature together. Editor's Choice, Science, 15 Apr 2016. dx.doi.org/10.1126/science.352.6283.305-a

\section{Attendance of conferences}

3D HTS and profiling of Embryoid Bodies in thermoformed microwell plates. Oral presentation. NBTE, Lunteren, The Netherlands, 2015

New Frontiers symposium - regenerative medicine, Nijmegen, The Netherlands, 2014

A microwell platform for High-Throughput Screens of embryoid bodies using small molecules. Poster at EMBO conference series. Erik J. Vrij, Nicolas C. Rivron, Roman Truckenmüller, Niels Geijsen, Clemens A. Van Blitterswijk. EMBL Chemical Biology, Heidelberg, Germany, 2012 
Poster. MIRA: Engaging reflection - the interface of biomedical technology and philosophy, Enschede, The Netherlands, 2011

Poster. Agarose Microwell-based High Throughput Screening Platform for Multicellular Aggregates. Erik J. Vrij, Nicolas C. Rivron, Roman Truckenmüller, Niels Geijsen, Clemens A. Van Blitterswijk. NBTE, Lunteren, The Netherlands, 2011

Agarose microwell high-throughput platform for wide screening of multicellular aggregates. Flash presentation including poster. Erik J. Vrij, Nicolas C. Rivron, Roman Truckenmüller, Niels Geijsen, Clemens A. Van Blitterswijk. BioNanoTec Montreux, Montreux, Switzerland, 2011

\section{Courses}

Using HTS to improve models of embryonic development in vitro. Poster at EMBL workshops. Erik J. Vrij, Nicolas C. Rivron, Roman Truckenmüller, Niels Geijsen, Clemens A. Van Blitterswijk. Workshop - Computational Aspects of High-Throughput Screening, Heidelberg, Germany, 2013

\section{Granted proposals}

KNMF standard proposal for Hot Embossing/Compression Molding. Title: Thermoforming PolyCarbonate films for 96-wells titre-plate-compatible high-throughput screening platform for wide screening of multicellular aggregates. Proposer: Erik J. Vrij, Proposal-ID: 2011-007-000687.

EUMINAfab Application Standard call for Diamond micromachining and polymer imprinting. Title: Development of a HTS platform for screening tissue developmental models in vitro.

\section{Patents}

Self-assembling tissue modules (WO2009154466). Inventors: Nicolas Clément Rivron, Jeroen Rouwkema, Roman Truckenmüller, Séverine le Gac, Clemens Antoni van Blitterswijk, Erik Jacob Vrij. Publication date: 23 December 2009

Blastoid, cell line based artificial blastocyst (WO2014171824A1). Inventors: Nicolas Clement Rivron, Clemens Antoni van Blitterswijk, Niels Geijsen, Erik Jacob Vrij. Publication date: 23 October 2014 
Acknowledgements

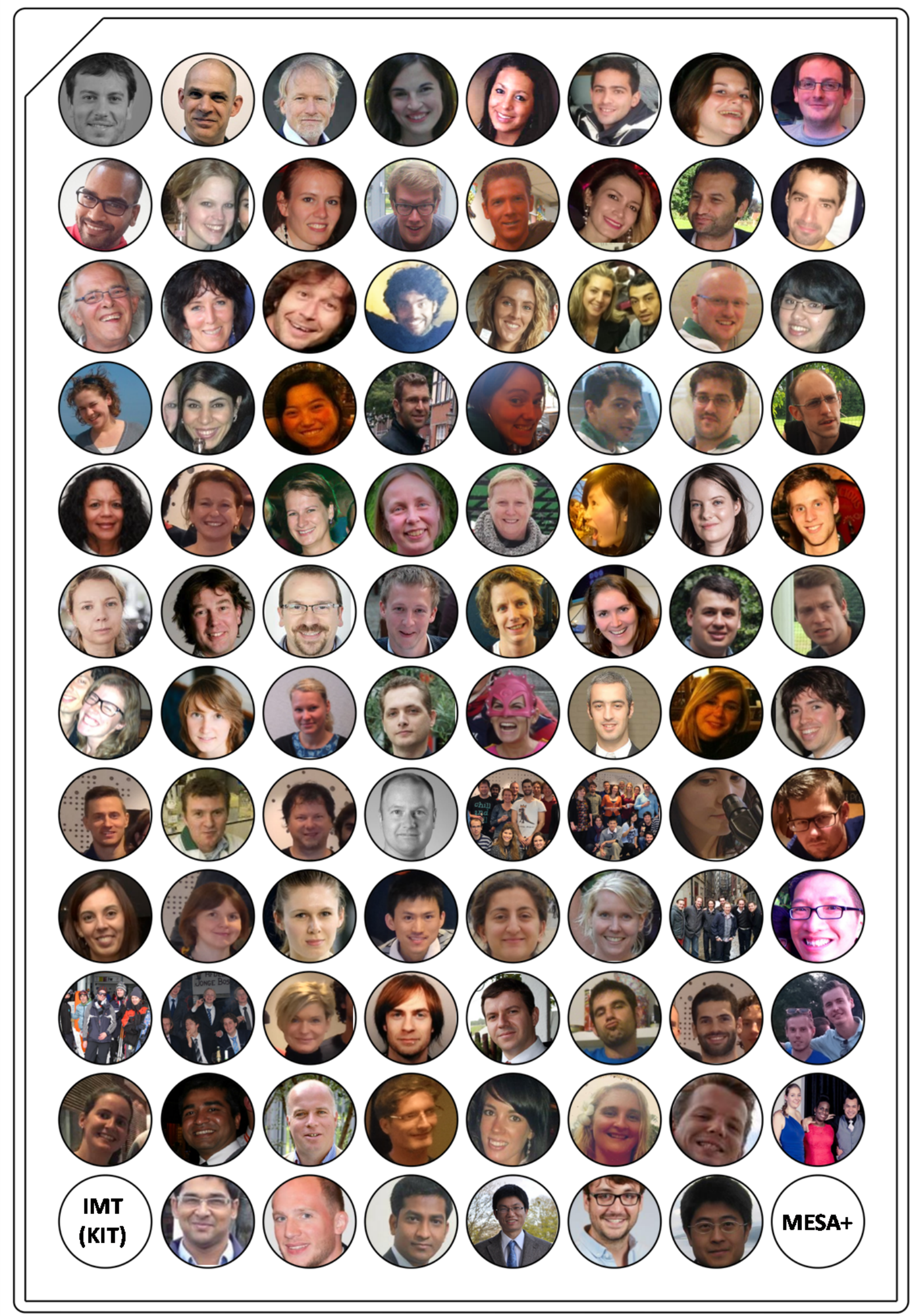


